University of Louisville

ThinkIR: The University of Louisville's Institutional Repository

$12-2018$

\title{
Coagulation Factor XIIIA: biochemical properties underlying physiological function.
}

Boris Anokhin

University of Louisville

Follow this and additional works at: https://ir.library.louisville.edu/etd

Part of the Biochemistry Commons

\section{Recommended Citation}

Anokhin, Boris, "Coagulation Factor XIIIA: biochemical properties underlying physiological function." (2018). Electronic Theses and Dissertations. Paper 3090.

https://doi.org/10.18297/etd/3090

This Doctoral Dissertation is brought to you for free and open access by ThinkIR: The University of Louisville's Institutional Repository. It has been accepted for inclusion in Electronic Theses and Dissertations by an authorized administrator of ThinkIR: The University of Louisville's Institutional Repository. This title appears here courtesy of the author, who has retained all other copyrights. For more information, please contact thinkir@louisville.edu. 


\title{
COAGULATION FACTOR XIII A: BIOCHEMICAL PROPERTIES UNDERLYING PHYSIOLOGICAL FUNCTION
}

\author{
By
}

Boris Anokhin

B.Sc., Perm State University, 2008

M.Sc. University of Louisville, 2018

\author{
A Dissertation \\ Submitted to the Faculty of the \\ College of Arts and Sciences of the University of Louisville \\ in Partial Fulfillment of the Requirements \\ for the Degree of
}

Doctor of Philosophy in Chemistry

Department of Chemistry

University of Louisville

Louisville, Kentucky

December 2018 
Copyright 2018 by Boris Anokhin

All rights reserved 



\title{
COAGULATION FACTOR XIII A: BIOCHEMICAL PROPERTIES UNDERLYING PHYSIOLOGICAL FUNCTION
}

\author{
By \\ Boris Anokhin \\ B.Sc., Perm State University, 2008 \\ M.Sc. University of Louisville, 2018
}

A Dissertation approved on

November 20, 2018

by the following Dissertation Committee:

Dissertation Chair

Dr. Muriel C. Maurer

Dr. Eugene G. Mueller

Dr. Richard J. Wittebort

Dr. Stanley E. D'Souza 


\section{DEDICATION}

This dissertation is dedicated to the late Dr. Lyudmila Anokhina, Professor of Medicine whose own scientific endeavor began with the investigation of serum proteins 


\section{ACKNOWLEDGEMENTS}

My immense gratitude is due to Dr. Muriel Maurer for the determined patience and essential support accompanying my writing of the opus majus, for the learning environment that for want of a better word I call comfortable.

I am very grateful to the members of Dissertation Committee Dr. Eugene Mueller, Dr. Richard Wittebort, and Dr. Stanley D'Souza for critical discussions and evaluation of the present work.

I deeply appreciate the expert participation of Dr. William Dean, Dr. Vilius Stribinskis, and Dr. Evgenia Moiseeva in this research project.

I treasure my warm friendship with current and past members of the Maurer Lab: Dr. Kelly Mouapi, Dr. Ramya Billur, Dino Ablan, Allison Davidson, Mohammed Hindi, Omair Ahmed, Lucille Wagner, Alex Gupta, Chad Stephens, Carmela Riposo, Blake Lynch, and Sara Zink.

I am thankful to my past mentors guiding me through the undergraduate journey Dr. Irina Ivshina, Dr. Tatyana Kamenskikh, Dr. Svetlana Tendryakova, Dr. Vitaliy Demakov, and Dr. Alexander Maksimov. 


\section{ABSTRACT \\ COAGULATION FACTOR XIII A: BIOCHEMICAL PROPERTIES UNDERLYING PHYSIOLOGICAL FUNCTION}

Boris Anokhin

November 20, 2018

Factor XIIIA (FXIIIA) is a transglutaminase that crosslinks intra- and extracellular protein substrates. FXIIIA is expressed as an inactive zymogen, and during blood coagulation, it is activated by removal of an activation peptide by thrombin. The resulting plasma transglutaminase forms isopeptide bonds between $\alpha$ and $\gamma$ chains of fibrin, thus stabilizing the clot. No such proteolytic FXIIIA activation is known to occur in other tissues (i.e. bone) or the intracellular form of FXIIIA. For those locations, FXIIIA is assumed instead to undergo activation by $\mathrm{Ca}^{2+}$ ions.

FXIIIA is the only transglutaminase existing as a dimer in zymogen form. The oligomeric state of the active enzyme remains controversial, and the present work commenced with addressing this issue. The results of size exclusion chromatography and analytical ultracentrifugation confirmed a dimeric state for zymogen and, for the first time, indicated a monomeric state for the active FXIIIA in solution. Analysis of FXIIIA mutants with impaired dimeric interfaces stressed the importance of dimeric state in maintaining zymogen stability in solution. Comparing sedimentation properties of proteolytically and nonproteolytically activated FXIIIA suggested conformational and functional differences 
between the two forms. Those differences were further assessed in a series of catalytic activity studies. Kinetic analysis revealed affinity for the glutamine substrate was higher for proteolytically activated FXIIIA than for FXIIIA activated by high $\mathrm{mM} \mathrm{Ca}^{2+}$.

FXIIIA proteolytic activation was investigated in a context of fibrin clotting. The V34X FXIIIA variants were generated using site-directed mutagenesis and recombinantly expressed in Escherichia coli. Those variants were ranked in terms of their activation rates by thrombin L34>>V34>F34>>W34. In a series of SDS-PAGE and clot turbidity assays, the proteolytic activation rates of FXIIIA variants were correlated with the extent of covalent fibrin crosslinking. The V34X FXIIIA variants were administered into murine FXIIIA-deficient plasma. Scanning electron microscopy of the whole plasma clots revealed morphological evidence for incorporation of nonfibrous protein into the clot. The magnitude of this incorporation correlated with the FXIIIA activation rate. Overall, the presented research fills in critical gaps in previously identified FXIIIA biochemical attributes and expands the understanding of FXIIIA physiological function. 
TABLE OF CONTENTS

ACKNOWLEDGEMENTS ..................................................................... iv

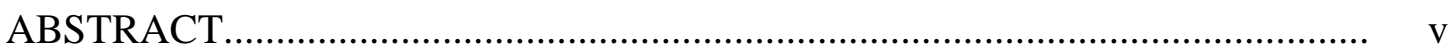

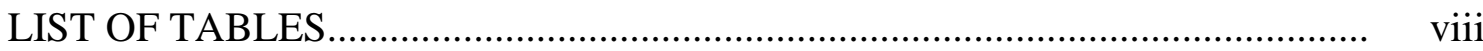

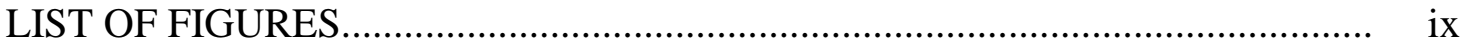

\section{CHAPTER}

1. INTRODUCTION ........................................................................ 1

2. A REVIEW OF SPECIAL TECHNIQUES EMPLOYED IN CURRENT RESEARCH PROJECT ......................................................................... 21

3. RECOMBINANT EXPRESSION OF FXIIIA IN ESCHERICHIA COLI AND SITE-DIRECTED MUTAGENESIS................................................. 38

4. PROBING OLIGOMERIC STATES OF FXIIIA IN SOLUTION................ 56

5. INVESTIGATION OF DYNAMIC AND FUNCTIONAL IMPLICATIONS OF FXIIIA ACTIVATION PATHWAYS.................................................. $\quad 79$

6. STUDYING THE EFFECCT OF FXIIIA ACTIVATION RATE ON FIBRIN CLOT ARCHITECTURE.......................................................................... 104

7. RESEARCH SUMMARY AND FUTURE DIRECTIONS........................... 124

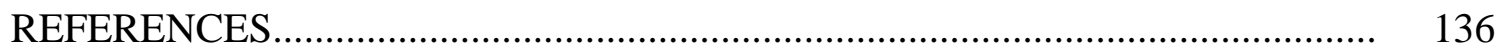

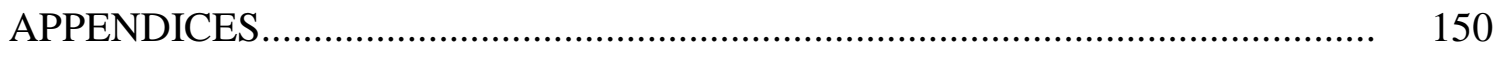

CURRICULUM VITAE......................................................................... 155 


\section{LIST OF TABLES}

\section{TABLE}

1. Primers used for PCR-based site-directed mutagenesis to obtain expression vectors for FXIIIA variants.

2. Primers used for PCR-based site-directed mutagenesis to obtain expression vectors for FXIIIA mutants........................................................................ 62

3. Hydrodynamic parameters of FXIIIA .......................................................... 66

4. Fibrin turbidity parameters............................................................... 112 


\section{LIST OF FIGURES}

\section{FIGURE}

1. Blood coagulation cascade....................................................................... 3

2. Physiological niche of FXIIIA................................................................. 10

3. Mechanism of the transglutaminase reaction............................................... 11

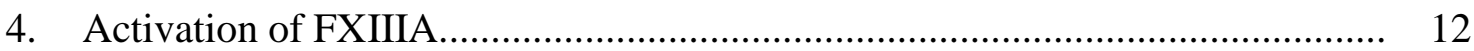

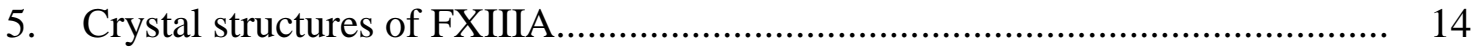

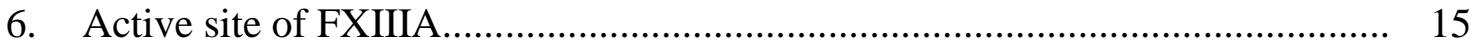

7. Amino acid residues in FXIIIA whose substitutions are believed to impair dimerization of the zymogen........................................................... 17

8. Schematic representation of fibrin fiber formation...................................... 19

9. Schematic of the Beckman Optima XL-A ultracentrifuge.............................. 23

10. Schematic of a sedimentation velocity AUC experiment................................ 26

11. Direct boundary modeling of sedimentation velocity data in the program

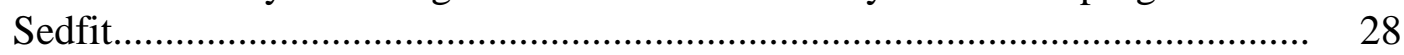

12. Graphical analysis of sedimentation equilibrium data................................. 30

13. Analysis of sedimentation equilibrium data in the program Sedphat................ 31

14. Simplified representation of the interaction volume..................................... 33

15. Principal schematic of a scanning electron microscope.................................. 35

16. A pGEX-6P-1 plasmid DNA map.......................................................... 43

17. Purification of FXIIIA and PSP recombinantly expressed using Protocol I....... 48

18. Purification of FXIIIA and PSP recombinantly expressed using Protocol II..... 50

19. Coupled activity assay of recombinantly expressed FXIIIA AP variants.......... 52

20. Intrinsic tryptophan fluorescence, nPAGE, and size exclusion chromatography analyses of FXIIIA...................................................... 64

21. Representative sedimentation velocity profiles of FXIIIA............................. 66

22. Analysis of monomer-dimer distribution of FXIIIA forms.......................... 68

23. Sedimentation velocity analysis of covalently inhibited FXIIIA..................... 69 
24. Analysis of recombinantly expressed FXIIIA mutants with proposed impaired dimerization of the zymogen.................................................................. 70

25. Analysis of recombinantly expressed FXIIIA mutant Y283C........................ 72

26. Analysis of monomer-dimer distribution of FXIIIA partially activated by thrombin.

27. FXIIIA dissociation as a function of $\mathrm{Ca}^{2+}$ concentration................................ 86

28. Dynamics of FXIIIA dissociation in the presence of 4 and $25 \mathrm{mM} \mathrm{CaCl}_{2} \ldots \ldots . . \quad 88$

29. Comparison of $\mathrm{c}(s)$-distributions for activated FXIIIA .................................. 90

30. Coupled ammonia release assay of FXIIIA transglutaminase activity.............. 91

31. DMPDA kinetic assay of FXIIIA transglutaminase activity.......................... 93

32. MDC assay of FXIIIA transglutaminase activity ..................................... 97

33. Thrombin-catalyzed cleavage of full length FXIIIA....................................... 109

34. An SDS-PAGE assay to follow covalent fibrin crosslinking by FXIIIA AP variants

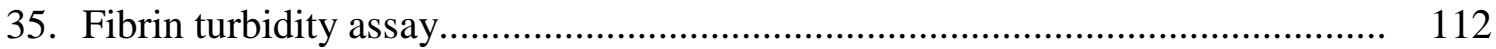

36. SEM analysis of fibrin clots fixed with glutaraldehyde............................... 114

37. SEM analysis of fibrin clots............................................................... 115

38. SEM of clots formed with whole plasma from WT mice................................ 117

39. SEM of clots formed with whole plasma from FXIIIA-deficient mice and exogenous FXIIIA...... 


\section{CHAPTER 1}

\section{INTRODUCTION}

Coagulation Factor XIII A (FXIIIA) is a member of the transglutaminase family of enzymes (protein glutamine:amine $\gamma$-glutamyltransferase, EC 2.3.2.13) that crosslinks protein substrates via an isopeptide bond in a $\mathrm{Ca}^{2+}$-dependent manner. FXIIIA has been a subject of biochemical research for almost a century. As physiological knowledge on FXIII expands, it becomes apparent that beyond serving as a key player of the coagulation system, FXIIIA functions in wound healing, bone tissue dynamics, and even signaling processes $(1,2)$. Being involved in an array of intra- and extracellular events, FXIIIA is implicated in associated pathological conditions and has thus become an attractive target for pharmacological intervention. Developing therapeutic regimes that effectively manage FXIIIA in its particular physiological environments definitely warrants a more in depth understanding of FXIIIA function in such locations. The purpose of this introductory chapter is to define the physiological niche of FXIIIA and to provide a brief overview of FXIIIA structural attributes. Goals of the current research project are presented in the conclusion.

FXIIIA is expressed as an inactive zymogen in cells of bone marrow origin, predominantly in megacaryocytes, monocytes, and macrophages (2-5). The intracellular distribution and functions of FXIII have been studied extensively during the last three 
decades (5). Megakaryocytes synthesize a large amount of FXIIIA. In the early stage of megakaryocyte development, FXIIIA is diffusely distributed in the cytoplasm. As the megakaryocyte maturates, FXIIIA is predominantly localized in a peripheral part of the cytoplasm and is then packaged in large quantities into the budding platelets. At the late megakaryocyte stage, FXIIIA is present in significantly lower quantities (6).

Monocytes also produce FXIIIA, and as they differentiate into macrophages, the FXIIIA expression levels increase (7). In macrophages, FXIII accumulates around cytoplasmic vacuoles and in pseudopods (8) and even appears in the nucleus (9). Nuclear FXIIIA is thought to participate in chromatin remodeling and cell differentiation. In the cytoplasm, certain cytoskeletal proteins serve as FXIIIA substrates, particularly actin and myosin, which are elements of the contractile locomotory system of the cells. The cytoskeletal system is involved in the phagocytosis function of monocytes and macrophages (5).

The first described and most studied function of FXIII is as a blood coagulation factor. Histologically, blood is viewed as a specialized connective tissue. Red blood cells (RBC, erythrocytes), white blood cells (leukocytes), and platelets (thrombocytes) constitute formed elements, and plasma is a liquid extracellular medium of this tissue. Since the blood circulates under pressure in the vascular system, an impairment to the integrity of that system, such as damage to a blood vessel, would result in bleeding. Provided the damage is not severe, the clotting system prevents excessive blood loss. This system is comprised of twelve coagulation factors (10), of which eleven are soluble plasma proteins (factors I - III, V, and VII - XIII; there is no factor VI) and one is the $\mathrm{Ca}^{2+}$ ion 
(factor IV).

The protein factors circulate in plasma as inactive precursor forms and may be activated in two different ways: the tissue factor (TF) pathway and the contact pathway (Fig. 1) (10). Tissue damage results in exposure of the TF expressing cells to the blood plasma. TF forms a complex with coagulation factor VIIa, which then activates factor IX through limited proteolysis. Factor IX in turn activates factor $\mathrm{X}$, through proteolysis as well. The TF pathway is also called the extrinsic pathway, because it requires interaction with the extrinsic TF. The contact (intrinsic) pathway is triggered without presentation of the TF. This pathway is initiated when plasma comes in the contact with a certain type of surface. Such interaction results in proteolytic activation of coagulation factor XII and the downstream factors, including factor $\mathrm{X}$.

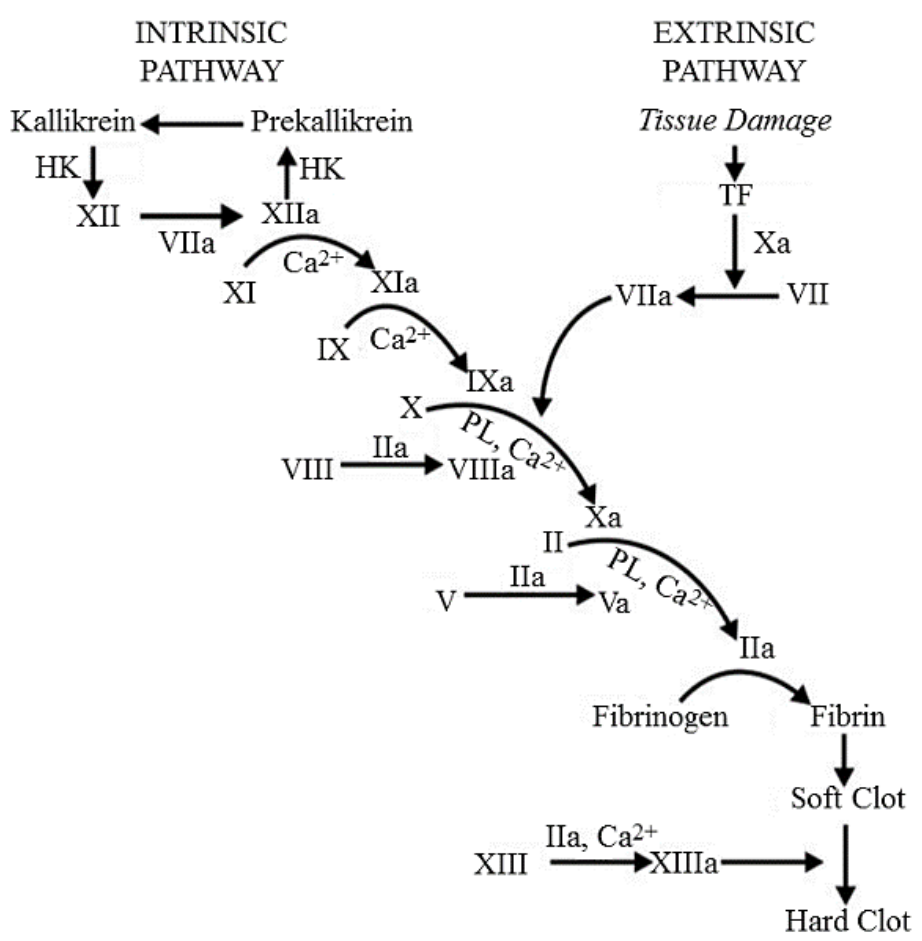

Fig. 1. Blood coagulation cascade. Roman numerals represent coagulation factors, letter 'a' denotes activated factors. HK - high molecular weight kininogen, TF - tissue factor, PL - phospholipid. Adapted from www.enzymeresearch.com. 
Thus, it could be seen that once the coagulation process is activated by one of the pathways, a series of proteolytic events then leads to generation of factor X. Factor X activates a serine protease thrombin (factor IIa). Thrombin proteolytically converts fibrinogen (factor I) to fibrin $(11,12)$, and in parallel, activates FXIIIA $(4,13,14)$. As the soluble fibrin undergoes self-polymerization into an insoluble fiber network, the transglutaminase FXIIIA introduces covalent crosslinks between the fibrin molecules within this network, thereby increasing its mechanical stability $(2-4,15)$. FXIIIA also crosslinks other proteins into the fibrin network; fourteen FXIIIA protein substrates were identified in clot proteomics (16). Importantly, FXIIIA-mediated incorporation of $\alpha_{2}$ antiplasmin imparts resistance of the fibrin network to fibrinolysis by the protease plasmin $(2,3,15)$. The fibrin net retains RBC that further increase overall mechanical stability of the blood clot and reduce its porosity. Entrapment of those cells in the clot has been strongly correlated with FXIIIA activity (17); however, no evidence of actual RBC crosslinking to the fibrin matrix has been reported. It is likely, therefore, that the $\mathrm{RBC}$ retention is mediated by the mechanical stability of the crosslinked fibrin network preventing escape of RBC with the incessant blood flow. Platelets are also incorporated in the fibrin clot but, unlike $\mathrm{RBC}$, they play a more active role in the clot dynamics by coordinating the clot retraction. Their surface protein, the integrin $\alpha_{\mathrm{II}} \beta_{3}$, anchors onto the fibrin mesh. As the platelet internal cytoskeleton undergoes reorganization (a FXIIIA-mediated process), it transmits the contractile force onto the extracellular fibrin fibers. This process ultimately results in reducing the clot volume due to compaction $(18,19)$. The compressed clot is even more impenetrable and provides a temporary plug that seals the damaged vessel.

The extrinsic TF coagulation pathway is proposed to dominate in the early stages 
of bleeding cessation upon vascular tissue damage. The intrinsic contact pathway has been regarded as part of mostly pathological processes (10); however, a recent review suggests its physiological role during the late stages of coagulation (19). After the plateletmoderated clot retraction, a series of tissue repair events occur. In these events, FXIIIA crosslinks extracellular matrix (ECM) proteins fibronectin, vitronectin, thrombospondin, and collagen, thus providing the scaffold for vessel repair. Furthermore, FXIIIA-catalyzed crosslinking of the vascular endothelial growth factor receptor to the integrin $\alpha_{v} \beta_{3}$ at the endothelial cell surface promotes cell motility and proliferation, thus initiating angiogenesis $(2,20,21)$.

At a later stage of angiogenesis, the crosslinked protein scaffold must undergo degradation to finalize tissue repair. The fibrinolytic system with the key player serine protease plasmin is responsible for this degradation $(22,23)$. The activation of plasmin is enhanced at the fibrin surface (23). At the same time, the efficiency of plasmin-mediated fibrinolysis strongly depends on the density of the fibrin clot. This dependence is two-fold: the contracted clots are less penetrable for plasmin and the concentration of the crosslinked plasmin inhibitor $\alpha_{2}$-antiplasmin is higher in a denser clot (18). FXIIIA function enhances fibrin density and increases incorporation of $\alpha_{2}$-antiplasmin and, therefore, has an impact on activity of the fibrinolytic machinery (24).

Described so far is a normal physiological presentation of blood coagulation, known as hemostasis. Coagulation may also proceed inside the blood vessels as part of pathological process called thrombosis (10). Thromboses account for the major part of coagulation-associated morbidity and mortality (15,17,24-27). Intravascular coagulation 
may occur in arteries and veins, and the central etiological event in both types of thrombosis is the deposition of fibrin. FXIIIA contribution to venous thrombosis is coagulation-related and results in stabilization of the thrombus. In arterial thrombosis, FXIIIA involvement is somewhat more complicated. Similar to venous thrombosis, the crosslinking activity occurring in the arterial thrombogenesis defines the FXIIIA prothrombotic effect. However, the FXIIIA function in post-thrombotic arterial events, such as myocardial infarction, has been correlated with better outcomes. These ameliorations are promoted by FXIIIA-mediated repair of the infarcted tissue.

The intrinsic (contact) pathway of coagulation is activated on the surface of certain bacteria entering the bloodstream (28-30). The resultant FXIIIA activity thus ultimately leads to sequestration of an infectious agent within a fibrin clot and prevents its spreading from the site of infection (21,31). This FXIIIA function is in part fulfilled by creating a stronger fibrin network. In addition, FXIIIA directly crosslinks the bacterial cells to fibrin, through specific surface proteins in a serotype-dependent manner. A good illustration of the FXIII protective role was provided by a study (32) where FXIII deficient mice developed a more severe systemic inflammatory response to some streptococcal infections. This severity was reduced upon administration of exogeneous FXIIIA. While in general FXIIIA activity prevents the spreading of pathogens from the infection site, a fascinating exception is Staphylococcus aureus. A majority of clinical S. aureus strains produce a hemostatic factor coagulase whose ability to bind fibrinogen and to activate thrombin results in fibrin deposition at the infection site $(33,34)$. Confined within the fibrin clot, S. aureus is protected from the host innate immune system and, unlike opportunistic 
pathogens, utilizes the fibrin matrix for replication and persistence, thus taking advantage of the coagulation system as part of its virulence strategy (35).

Systemic inflammation also activates the coagulation cascade. Extreme inflammatory complications of infection, such as sepsis, result in hyperactivity of the clotting system (28). In a proteomic study, almost the entire complement inflammatory system has been shown to be a target for FXIIIA (16). However, the reports examining FXIIIA interaction with those substrates are scarce. In general, increased levels of FXIIIA crosslinking result in extensive fibrin deposition throughout the microvasculature. This condition, referred to as disseminated intravascular coagulation (DIC), results in cessation of the organ blood supply and ultimately, organ failure (36). At the same time, ongoing consumption of the clotting factors and platelets in DIC together with hyperfibrinolysis, may result in excessive bleeding. Dysregulation of the coagulation cascade also plays an etiologic role in other inflammatory diseases including asthma, rheumatoid arthritis, etc., through excessive deposition of fibrin (37-41).

Coagulation is tightly interconnected with oncological events (42-44). Tumor cells constitutively express TF and expose it on the surface thereby activating the coagulation system. Tumor procoagulant activity is also manifested through expression of a so-called cancer procoagulant that directly activates factor X. Thrombotic events significantly contribute to malignancy-associated complications, and the risk of developing venous thrombotic embolism in cancer patients is increased seven-fold compared to the general population. Analogous to septic complications, DIC-related severe hemorrhages are often 
observed in cancer. The DIC is particularly associated with acute leukemias.

Besides the devastating thrombotic and hemorrhagic complications, the coagulation system is implicated in tumor neoangiogenesis. The mechanism of coagulation system neoangiogenic function is essentially the same as outlined above for post vascular injury events, except here it becomes a part of the tumor progression. Localized fibrin deposition enhances adhesion of the tumor cells to the endothelium, facilitating the metastatic process. Furthermore, the tumor cells activate platelets, and the resulting tumor cell-platelet thrombi support metastasis formation by shielding the tumor from the innate immune system, an outcome similar to that aforementioned for S. aureus.

Apart from serving in the coagulation system, FXIIIA fulfills coagulationindependent functions. Although not recognized enough and even less understood, a unique place for FXIIIA is in the extracellular matrix of osseous tissue. The bone mass is regulated by the bone tissue remodeling. This remodeling occurs constantly at 1-2 million sites in an adult skeleton (45). Coordination of this process involves multiple physiological pathways that are beyond the scope of this chapter. In a simplified approach, the bone dynamics involves reciprocal activity of bone-resorbing osteoclasts and bone-forming osteoblasts (46). Remodeling is initiated by osteoclasts secreting $\mathrm{HCl}$ into the resorptive space, yielding an extracellular $\mathrm{pH}$ of $<4.5$ and very high (on the order of $40 \mathrm{mM}$ ) local concentrations of $\mathrm{Ca}^{2+}$. In response to such $\mathrm{Ca}^{2+}$ levels, osteoblasts migrate to the site of bone resorption (45). Osteoblasts generate ECM that forms a substratum to support cell attachment and regulates mineralization. The bone ECM consists primarily of type 1 collagen (COL1), and plasma fibronectin $(\mathrm{pFN})$ is a major component of noncollageneous 
bone matrix (47-49).

The reports on FXIIIA function in the bone remodeling are somewhat contradictory. They agree, however, that functions of FXIIIA and Transglutaminase 2 (TG2) in the bone ECM overlap. A single deficiency of either protein did not result in an overt bone phenotype in murine models, which was attributed to a compensatory effect of the other transglutaminase (50-52). A study employing murine model of double FXIIIA and TG2 deficiency concluded that neither transglutaminase was required for bone mineralization (53). Moreover in another study, inhibition of the transglutaminase activity was associated with reduced osteoclastogenesis and better outcome in collagen induced arthritis (41). However, a work examining the maintaince of bone mass and remodeling activity of bone cells, revealed that together with TG2, FXIIIA was a negative regulator of osteoclastogenesis. Absence of TG2 and FXIIIA led to overexpression of osteoclastogenesis-promoting transglutaminase 1 , whose inhibition likely reduced bone loss in the pathogenesis of arthritis $(49,54)$.

Thus, FXIIIA activity is currently known to mediate accumulation and crosslinking of the $\mathrm{pFN}$ in the bone ECM (55). The $\mathrm{pFN}$ accumulation enhances proliferation and differentiation of the osteoblasts. Plasma membrane-associated FXIIIA promotes secretion of COL1 by osteoblasts (56). Extracellularly, FXIIIA and TG2 coordinate COL1 matrix deposition thereby promoting bone mineralization $(49,51,54,56,57)$.

A cartoon in Fig. 2 summarizes some aspects of the physiological presence of FXIIIA in cells, bone tissue, and blood plasma. 


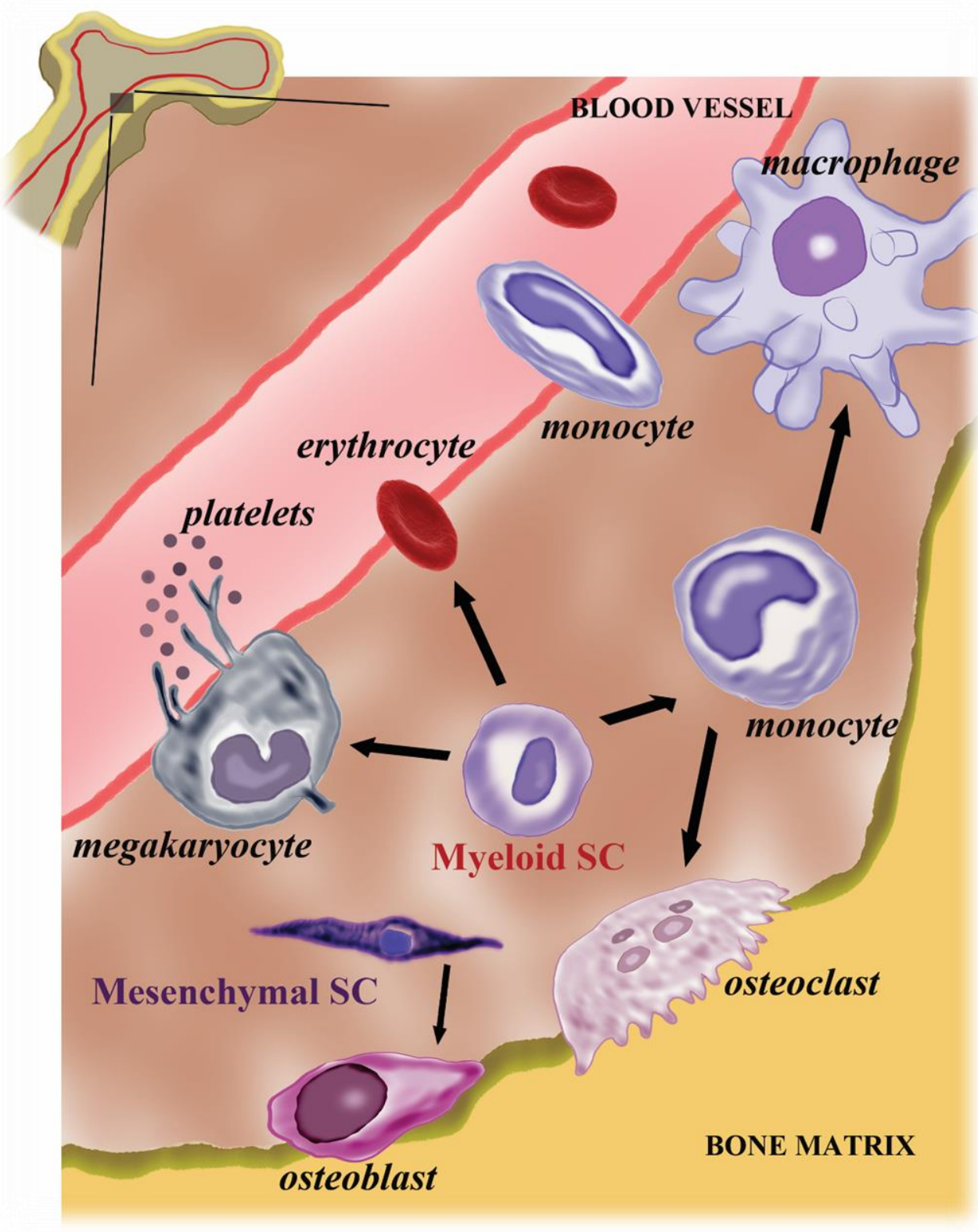

Fig. 2. Physiological niche of FXIIIA. FXIIIA begins its journey in the cells of bone marrow. The myeloid stem cells undergo differentiation events (denoted by arrows): erythropoiesis that results in formation of red blood cells and thrombopoiesis resulting in megakaryocytes. Megakaryocytes synthesize large amount of FXIIIA and package it into the platelets. Leukopoiesis leads to formation of lymphocytes, granulocytes and monocytes, with the latter being the most relevant to the subject of present review. FXIIIA is the only transglutaminase expressed in monocytes, and the ruptured monocytes have been assumed to serve as a source of plasma FXIIIA. The FXIIIA expression increases during differentiation of macrophages. On the contrary, differentiation of osteoclasts is accompanied by decrease in FXIIIA levels. As part of tissue remodeling, osteoclasts mediate bone resorption. Osteoblasts differentiate from the mesenchymal stem cells, and during that differentiation FXIIIA expression is upregulated. Osteoblasts are responsible for the deposition of ECM and subsequent mineralization of bone. 


\section{Current view on the structure and activation of FXIIIA}

As mentioned in the beginning of this chapter, FXIIIA has been a subject of biochemical research for almost a century. The first report suggesting covalent stabilization of fibrin appeared in 1923. Barkan and Gaspar (Barkan G., Gaspar A. Zur Frage der Reversibilitä t der Fibringerinnung II. Biochem. Ztschr. 1923, from (1)) observed that the fibrin clots formed in the presence of $\mathrm{Ca}^{2+}$ became insoluble in weak bases. A couple of decades later, Robbins proposed the existence of a "serum factor" rendering the clots insoluble (Robbins K.C. A study on the conversion of fibrinogen to fibrin. Am. J. Physiol. 1944, from (1)). Laki and Lorand (58) later named the protein "fibrin stabilizing factor". The name Factor XIII appeared in the literature in the 1960s.

FXIIIA catalyzes formation of an $\varepsilon$-( $\gamma$-glutamyl) lysine isopeptide bond, following a modified ping-pong mechanism shared by members of the transglutaminase family $(59,60)$ (Fig. 3).

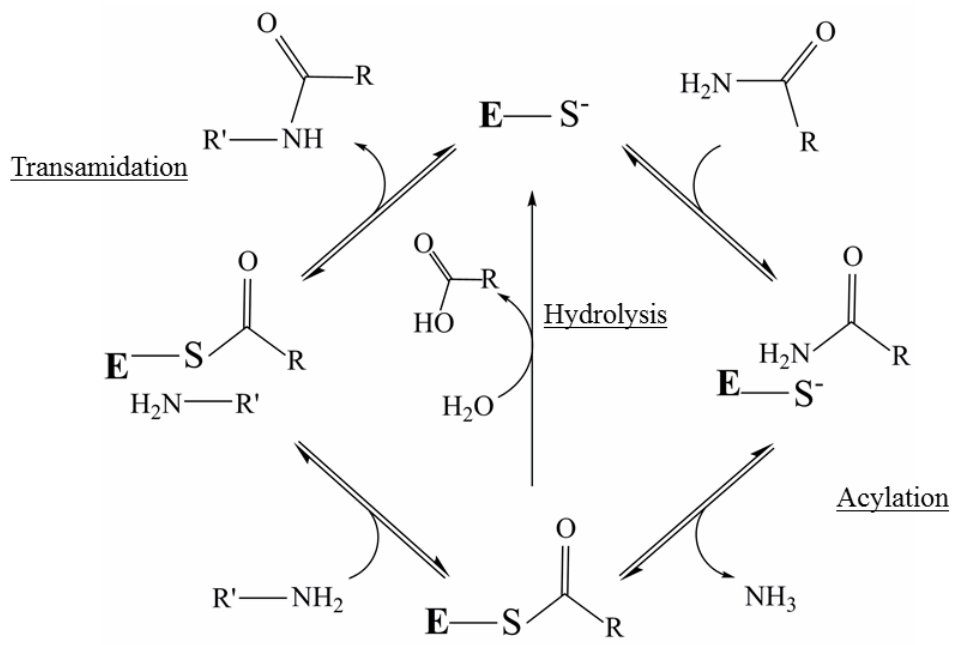

Fig. 3. Mechanism of the transglutaminase reaction. During the first half-reaction the thiolate of catalytic cysteine performs a nucleophilic attack on the carbonyl carbon of glutamine side chain, resulting in formation of a thioester acyl-enzyme intermediate and release of ammonia. Deacylation and regeneration of the free enzyme may proceed through acyl transfer to a primary amine such as lysine side chain (transamidation) which results in the formation of an isopeptide bond between the glutamine and amine substrates. In the absence of amine substrate, the thioester intermediate undergoes hydrolysis resulting in net deamidation of the glutamine to glutamate. Adapted from (59). 
Unlike most other transglutaminases, FXIIIA is expressed as an inactive zymogen, and in plasma, it circulates in a $\sim 325 \mathrm{kDa}$ heterotetrameric complex with carrier FXIIIB $\left(\mathrm{A}_{2} \mathrm{~B}_{2}\right.$, the molecular weight of each A-subunit is $\sim 83 \mathrm{kDa}$, and each FXIIIB molecule is $\sim 79 \mathrm{kDa}$ ). During blood coagulation, FXIII is activated by thrombin-mediated removal of N-terminal activation peptides from the A-subunits followed by dissociation of the B-subunits (61) in the presence of $\mathrm{Ca}^{2+}$ (Fig. 4).

FXIIIA exists as an $\mathrm{A}_{2}$-homodimer within the cells and, while intracellular FXIIIA activity does figure in multiple reports, its activation in this physiological compartment is much less understood. No intracellular proteolytic modification of FXIIIA has been found, and the current consensus is a slow nonproteolytic activation in the presence of low available $\mathrm{Ca}^{2+}$ concentrations (62-64). Both plasma and cellular FXIII can be activated non-physiologically in the presence of high $(>50 \mathrm{mM}) \mathrm{Ca}^{2+}$ (Fig. 4) Lorand (65) demonstrated that $\mathrm{Ca}^{2+}$ is crucial for the transglutaminase activity, whereas other divalent cations were much less effective, in the order $\mathrm{Sr}^{2+}>\mathrm{Ba}^{2+}>\mathrm{Mg}^{2+}$. This specific effect of $\mathrm{Ca}^{2+}$ could be augmented, but not replaced, by increasing ionic strength.

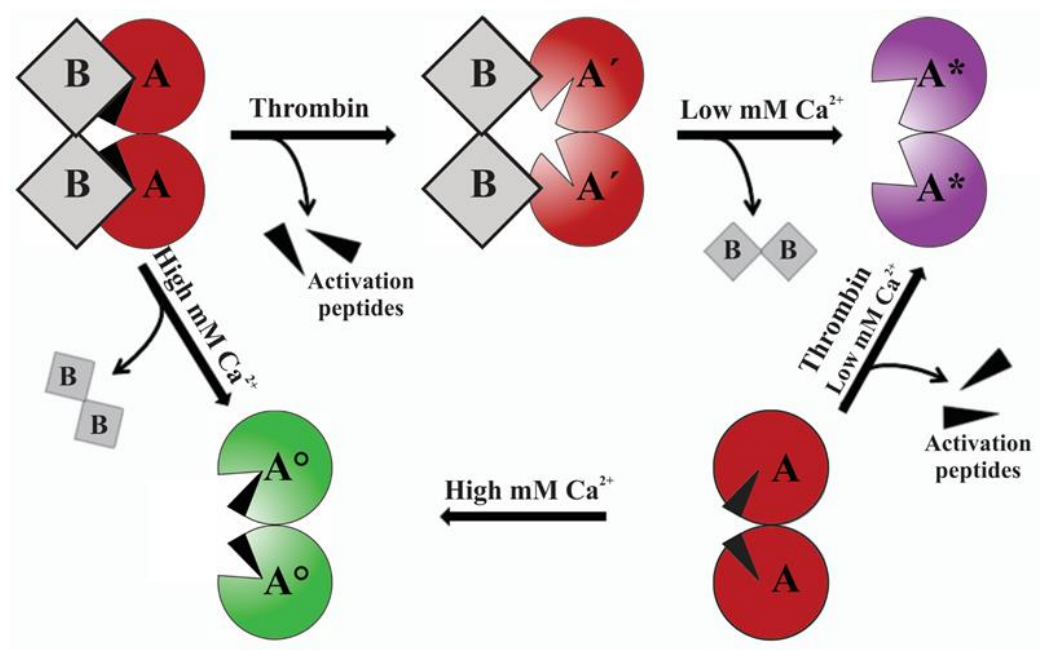

Fig. 4. Activation of FXIIIA. A ${ }^{\prime}$ - FXIIIA after cleavage of the activation peptide, $A^{*}$ - FXIIIA activated by thrombin in the presence of low $\mathrm{mM} \mathrm{Ca}^{2+}, \mathrm{A}^{\circ}-$ FXIIIA activated nonproteolytically in the presence of high $\mathrm{mM} \mathrm{Ca}^{2+}(66)$. 
The amino acid sequence of the active site region was identified by Holbrook and coworkers (67), and the full sequence was determined by Ichinose et al. (68). FXIIIA was the first member of the transglutaminase family whose crystal structure was solved and, noteworthy, this structure later served as a template for solving the structures of other members of the transglutaminase family. Consistent with previous solution studies, the structure by Yee and coworkers revealed two crystallographically independent A-subunits (Fig. 5A) (69,70). The structural domains of each A-subunit include the N-terminal activation peptide (residues 1-37), $\beta$-sandwich (38-183), catalytic core (184-515) containing conserved transglutaminase catalytic triad C314, H373, and D396, $\beta$-barrel 1 (516-627), and $\beta$-barrel 2 (628-730). As evident from their names, the $\beta$-sandwich and the two $\beta$-barrel domains present primarily $\beta$-elements of secondary structure. The catalytic core domain is predominantly comprised of $\alpha$-helices.

The A-subunits of FXIII are oriented in a head-to-tail fashion within the dimer. The interface between the monomers is long and involves the $\beta$-sandwich and the catalytic core domains, with a contact area of $\sim 1800 \AA^{2}$. The catalytic core domain of the zymogen Asubunit is shielded by the two $\beta$-barrels. The energy of interaction between the catalytic domain and $\beta$-barrel 1 is $-11 \mathrm{kcal} / \mathrm{mol}$, and a prominent feature of this interaction is a hydrogen bond between the sulfur of catalytic $\mathrm{C} 314$ and the hydroxyl group of the $\beta$-barrel 1 residue Y560. In addition, the catalytic site of each subunit is blocked by the activation peptide of the neighboring subunit. The energy of activation peptide interaction with its own subunit is approximately $-9 \mathrm{kcal} / \mathrm{mol}$ and about $-5 \mathrm{kcal} / \mathrm{mol}$ with the other subunit. Each A subunit in the FXIII zymogen contains a $\mathrm{Ca}^{2+}$ ion (not shown) coordinated by the main chain carbonyl oxygen of A457 and sidechain carboxylates of D348, E485, and E490. 
In addition, three cis-peptide bonds are found in FXIIIA: G410-P411, and two non-proline cis-bonds R310-Y311 and Q425-F426 (71). A cis-trans isomerization of these bonds was proposed to act as a conformational switch; however, no experimental evidence supporting this hypothesis has been reported.
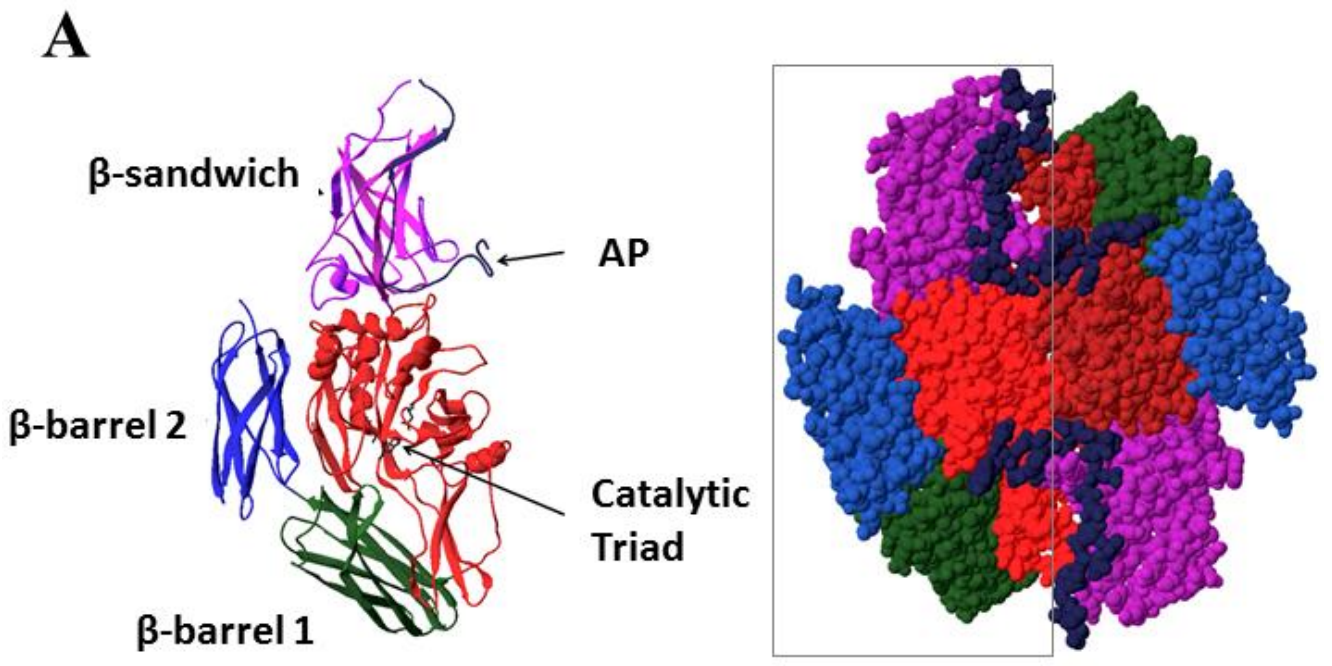

\section{B}
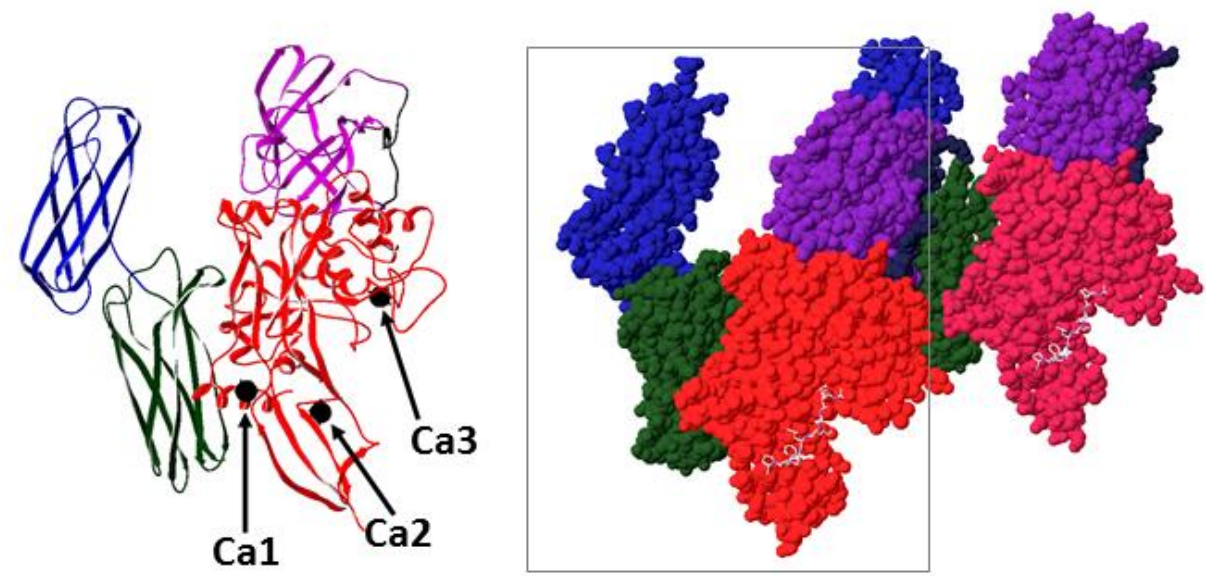

Fig. 5. Crystal structures of FXIIIA. (A) FXIIIA zymogen (PDB 1F13): the dimer is shown in a spacefilling model, and structural elements of the left subunit are highlighted in a ribbon representation; (B) non-proteolytically activated FXIIIA ${ }^{\circ}$ (PDB 4KTY): asymmetric crystal unit is shown in space-filling with covalently bound inhibitor ZED1301 in ball-and-stick, and a single subunit - as ribbons, with the three bound $\mathrm{Ca}^{2+}$ ions denoted as black spheres. ZED1301: Ac-(D)-Asp-MA-Nle-Nle-Leu-Pro-Trp-Pro$\mathrm{OH}$, (MA - Michaelis acceptor, Nle - norleucine). 
Recently, a high-resolution crystal structure of an activated FXIIIA with a glutamine mimic inhibitor bound in the active site was described by Stieler et al. (72). Unlike the zymogen, the structure of inhibited FXIIIA revealed that the two $\beta$-barrels and the activation peptide moved aside (Fig. 5B), exposing the catalytic center. Besides the previously observed $\mathrm{Ca}^{2+}$ in the zymogen, two additional $\mathrm{Ca}^{2+}$-binding sites were described. In addition, a hydrophobic tunnel formed by side chains of W279 and W370 was observed (Fig. 6). The proposed function of this pair was to reduce the possibility of hydrolysis of the thioester intermediate formed by catalytic C314 and the glutamine substrate. Molecular docking experiments indicated that the lysine side chain would fit perfectly into this tunnel.
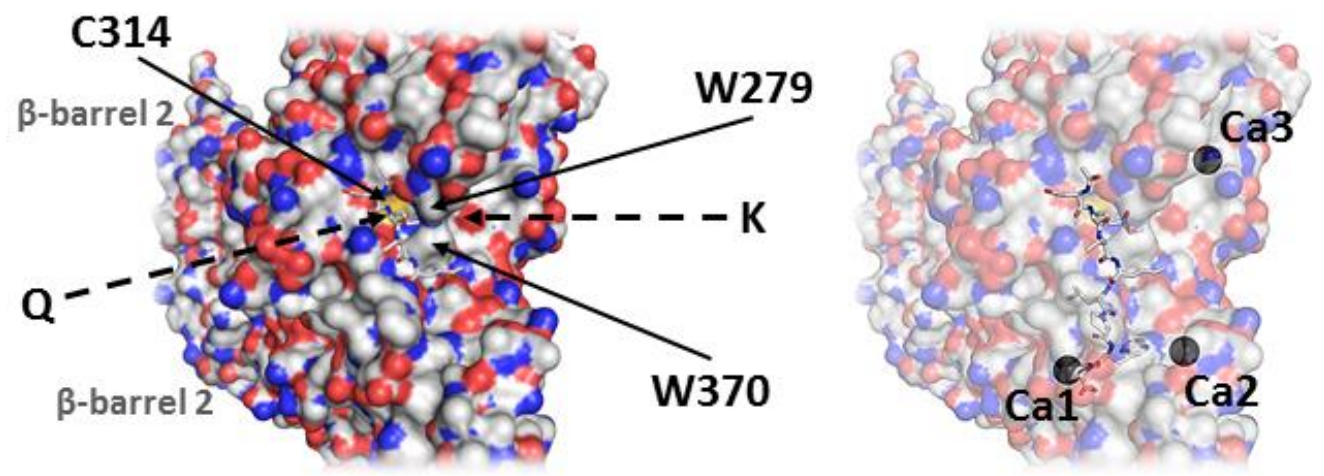

Fig. 6. Active site of FXIIIA. The zoomed in (PDB 4KTY) active site of the activated FXIIIA ${ }^{\circ}$ is displayed as a surface potential representation (Pymol) with the inhibitor depicted as ball-and-stick. W279 and W370 form a hydrophobic tunnel. Dashed arrows indicate the direction of entrance of the glutamine donor (Q) and the lysine donor $(\mathrm{K})$ substrates. Depicted on the right is identical view of the FXIIIA subunit with increased transparency for visualization of the buried $\mathrm{Ca}^{2+}$ ions and part of the inhibitor molecule.

Thus, along with structural features, this introductory review outlines FXIIIA roles in normal and pathological events. By no means does it cover the entire physiological involvement of FXIIIA. The overview, however, brings awareness that FXIIIA is not solely a blood coagulation factor. Such recognition significantly impacts the design of the presented research project. 


\section{RESEARCH GOALS}

The ultimate purpose of the present project was to understand FXIIIA activation. In particular, what global rearrangements does FXIIIA undergo during activation, and how may those rearrangements affect FXIIIA function in different physiological environments? The research can be divided into three major portions. First, FXIIIA oligomeric states were assessed in solution as a function of the activation state. Next, dynamic and functional implications of physiologically relevant FXIIIA activation pathways were investigated. Finally, a special case of FXIIIA activation was further studied in the context of a fibrin clotting model.

\section{Rationale for studying FXIIIA oligomeric states}

Zymogen FXIIIA exists as an $\mathrm{A}_{2}$ homodimer. In plasma, FXIIIA 2 is found in a complex with two carrier B-subunits $\left(\mathrm{A}_{2} \mathrm{~B}_{2}\right)$. In the coagulation cascade, thrombin cleaves the N-terminal activation peptide from the A-subunits of FXIII, followed by $\mathrm{Ca}^{2+}$ dependent dissociation of the B-subunits exposing catalytically active transglutaminase. FXIII $\mathrm{A}_{2}$ can be activated by thrombin in the presence of low $\mathrm{mM} \mathrm{Ca}^{2+}$. Both FXIII $\mathrm{A}_{2} \mathrm{~B}_{2}$ and $\mathrm{A}_{2}$ can be activated non-proteolytically by $>50 \mathrm{mM} \mathrm{Ca}^{2+}$. In early studies by Chung

and coworkers (73), thrombin-activated FXIIIA in the presence of $\mathrm{Ca}^{2+}$ appeared as a monomer in gel filtration, but it was concluded to exist in dimeric form from sedimentation equilibrium analysis. Thus, the active form of FXIIIA has long been considered a dimer. In a recent work (72), however, non-proteolytically activated FXIIIA with an inhibitor bound to the active site was proposed to exist as a monomer. The research discussed in Chapter 4 revisits the conformational rearrangements and oligomeric states of FXIIIA in a 
solution environment.

It is believed that single amino acid substitutions have the potential to disrupt dimer formation by the zymogen FXIIIA. A few potential candidates had been identified in patients with severe FXIII deficiency: R260L (74), R260C (75-77), Y283C and G562R (78) (Fig. 7). An R11Q substitution in the activation peptide (Fig. 7) was computationally predicted to impair $\mathrm{A}_{2}$ dimer formation (79). The experiments on the mutants were performed using cell lysates rather than purified proteins. A characteristic of all those mutants was an extremely low expression level in eukaryotic cell lines. Employing FXIIIA mutants as a model system, Chapter 4 discusses the importance for FXIIIA zymogen to form a correct dimer interface.

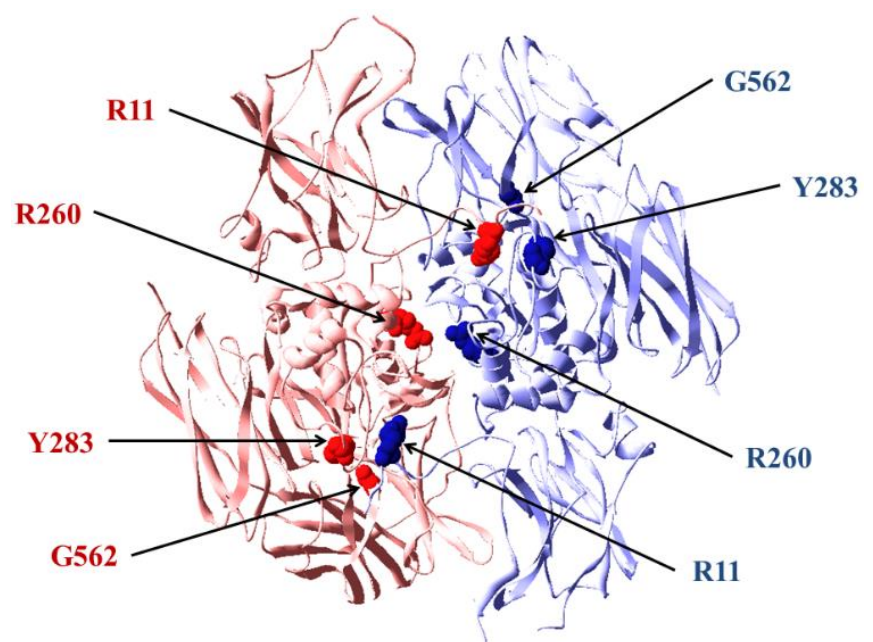

Fig. 7. Amino acid residues in FXIIIA whose substitutions are believed to impair dimerization of the zymogen. FXIIIA subunits are represented as ribbons, and selected residues - as molecular surfaces.

\section{Rationale for studying different mechanisms of FXIIIA activation}

The medical literature abounds with statistical research correlating FXIIIA levels with outcomes in numerous pathological conditions. Often, however, relatively new reports contradict conclusions of the previous publications. This issue is not unique for the FXIIIA field and may often be attributed to the absence of experimentally confirmed cause 
and effect relationships that underlie statistically significant correlations. The lack in establishing those relationships stresses, yet again, the incomplete understanding of FXIIIA activation and function.

Despite century-long research, FXIIIA is still viewed primarily as a coagulation factor. FXIIIA activation in plasma by thrombin was characterized relatively well even in early studies (80-82). Intracellular activation had only been studied for platelet FXIIIA (6264). Nonproteolytic activation of cellular FXIIIA was experimentally shown to proceed even in the presence of low $\mathrm{Ca}^{2+}$ levels; however, at nonphysiologically high $(1 \mathrm{M})$ concentrations of $\mathrm{Na}^{+}$or $\mathrm{K}^{+}$. No experimental insight is currently available on the dynamic and functional implications of low $\mathrm{mM} \mathrm{Ca}{ }^{2+}$-activation of FXIIIA.

FXIII activation by high ( $>50 \mathrm{mM}) \mathrm{Ca}^{2+}$, first described by Credo et al. (83), is regarded as being nonphysiological. Previous work by the Maurer group revealed different patterns of solvent accessibility for FXIIIA activated by thrombin and by high $\mathrm{mM} \mathrm{Ca}{ }^{2+}$ (84-86). Active FXIIIA could only be crystallized after nonproteolytic activation in the presence of high $\mathrm{Ca}^{2+}$ concentration followed by irreversible inhibition (72). Although being a static "snapshot", this crystal structure provided a valuable and previously unavailable view of active FXIIIA at the molecular level. The structure was later used for computational modeling of proteolytic FXIIIA activation (87). This strategy occurred despite previously reported structural differences between proteolytically and nonproteolytically activated forms. Chapter 5 investigates proteolytic and nonproteolytic activation of FXIIIA in solution, and provides insights on global conformational differences of the resulting FXIIIA forms.

FXIIIA in the ECM has recently begun to receive considerable attention, and a few 
published studies outline the role of FXIIIA in bone tissue dynamics $(47,49,54,55,57)$. In an early study, Kaartinen and coworkers proposed that a $37 \mathrm{kDa}$ proteolytic fragment was a major FXIIIA form in the bone ECM (47). However, Cordell et al. (53) later identified this fragment as transaldolase- 1 and demonstrated its immunoreactivity with anti-FXIIIA antibody. Thus far, no information is available on FXIIIA activation in this physiological compartment, and a possible activation mechanism is discussed in Chapter 5.

\section{Rationale for studying the effect of FXIIIA activation rate on fibrin architecture}

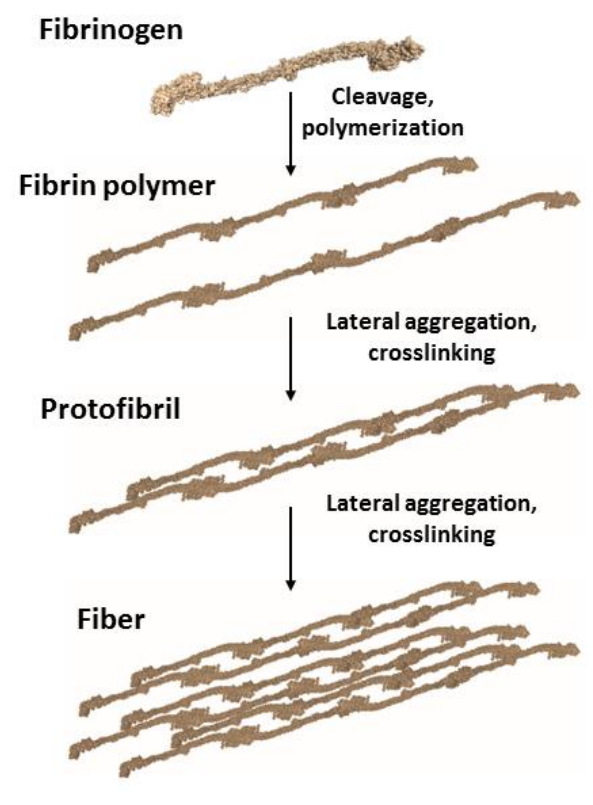

Fig. 8. Schematic representation of fibrin fiber formation
Fibrin is the main constituent of a blood clot. Its precursor, fibrinogen, represents a dimer of trimers: $(\mathrm{A} \alpha \mathrm{B} \beta \gamma)_{2}$. In the final stage of blood coagulation, thrombin cleaves fibrinopeptides A and B from fibrinogen, forming fibrin and initiating polymerization of fibrin monomers into fibers (Fig. 8). At the same time, activated FXIIIA catalyzes formation of covalent bonds between $\gamma-\gamma, \gamma-\alpha$, and $\alpha$ $\alpha$ chains of adjacent fibrin molecules. Crosslinking of $\alpha_{2}$-antiplasmin into the fibrin network makes it mechanically stronger and resistant to fibrinolysis.

Interestingly, a V34L polymorphism in the activation peptide of FXIIIA was associated with altered clot structure, more precisely, with thinner fibrin fibers (88). It was recently shown (89) that the L34 variant increased fibrin cross-linking in a murine model of thrombosis. A noticeable feature of the L34 variant is its enhanced cleavage by thrombin, 
leading to the faster activation of FXIIIA. Kinetic studies performed in the Maurer lab (9096) employed an HPLC assay on activation peptide fragments (residues 28-41) and confirmed that the L34 variant was hydrolyzed by thrombin more efficiently than the more common V34. A series of additional activation peptide substitutions was generated with an extended range of the cleavage rates. The rates of FXIIIA proteolytic activation were predicted to affect the properties of fibrin clot. The research in Chapter 6 utilizes full length V34X protein variants to test this prediction. 


\section{CHAPTER 2}

\section{A REVIEW OF SPECIAL TECHNIQUES EMPLOYED IN THE CURRENT RESEARCH PROJECT}

Biochemists are extremely fortunate to have a variety of methods at their disposal nowadays. Techniques such as gel electrophoresis, spectrophotometry, chromatography etc. have been routinely employed to study proteins, and the current project was not an exception. Two instrumental approaches employed in this work, analytical ultracentrifugation and scanning electron microscopy, are less frequently utilized in biochemical research and deserve a special discussion. The present brief review is intended to familiarize the reader with the principles behind these two techniques.

\section{Analytical Ultracentrifugation}

Analytical ultracentrifugation (AUC) is a universal method for characterization of macromolecules (proteins, nucleic acids, carbohydrates etc.). Being based on first principles of thermodynamics, AUC is considered a 'gold standard' method for determination of weight, hydrodynamic, and thermodynamic properties of biological molecules. The AUC is nondestructive and does not rely on sample interaction with a matrix. Although AUC is not a high throughput technique, multiple parameters can be obtained in a single experiment. Some current practical AUC applications include examination of sample purity, analysis of associating systems, detection of conformational 
changes, and ligand binding studies. Ongoing improvement of algorithms for AUC data analysis further increases experimental resolution, thus the list above is expected to expand.

The behavior of a macromolecule in a centrifugal field is affected by its weight, size, and shape. This sedimentation behavior is observed in AUC through a distribution of analyte concentration in the centrifugal cell. Such observation is made possible by introducing an optical system into a centrifuge. The common optical systems in modern analytical ultracentrifuges are based either on interference, absorbance, or fluorescence. The schematic representation of an Optima XL-A (Beckman Coulter) analytical ultracentrifuge equipped with absorbance optics is shown in Fig. 9.

A double sector centerpiece allows loading of sample and reference solution into two separate sector-shaped cells. This shape for the cells minimizes the convective disturbances resulting from collision with the wall of analyte molecules traveling along radial lines in the centrifugal field. The centerpiece is placed into the rotor and during centrifugation, a light source is briefly fired as the cells pass the detector, while a moving slit directs the absorbance scanning at different radial positions of the cell. Multiple scans acquired in an AUC experiment provide information on the molecular redistribution of the absorbing analyte during the course of the experiment. The high degree of optical precision and detector sophistication makes scanning through the entire length of the cell at ultrahigh (dozens of thousands revolutions per minute) centrifugation speed possible. That precision also applies to the control of rotor temperature, which is aided by evacuation of the centrifugation chamber to avoid warming of the fast spinning rotor due to friction with air. 


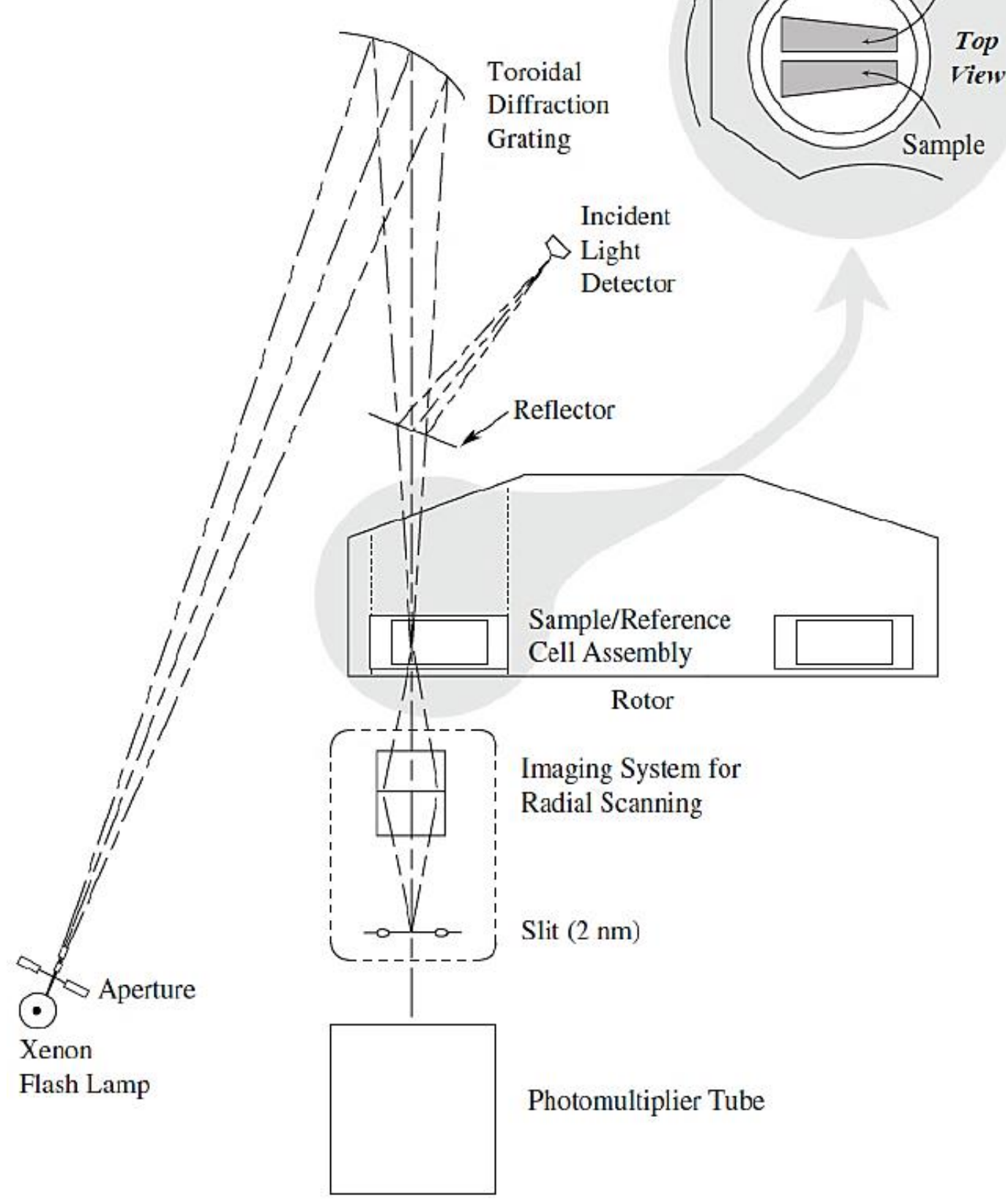

Fig. 9. Schematic of the Beckman Optima XL-A ultracentrifuge. Adapted from www.beckmancoulter.com. 
In the centrifugal field, a macromolecule (i.g., protein) is acted upon by the centrifugal $\left(F_{c}\right)$ force and two opposing forces, frictional $\left(F_{f}\right)$, and buoyant $\left(F_{b}\right)$. The $F_{c}$ is proportional to the mass of the macromolecule and the acceleration (reviewed in (97)):

$$
F_{c}=m \omega^{2} r=\frac{M}{N} \omega^{2} r
$$

where $m$ is the mass of a molecule, $M$ the molar mass of the solute, $N$ Avogadro's number, $r$ the distance of the macromolecule from the axis of rotation, and $\omega$ the rotor angular velocity. As the macromolecule accelerates in the direction of the centrifugal force, the solvent must flow around causing an increasing resistance to acceleration of the macromolecule. One component of this resistance is the frictional force. The frictional force depends on the velocity, size, and shape of the macromolecule:

$$
F_{f}=-f V
$$

where $V$ is the velocity and $f$ the frictional coefficient of the macromolecule that represents its size and shape. The second component of solvent resistance to the macromolecule acceleration under the centrifugal force is the buoyant force $F_{b}$, which is equal to the weight of displaced solvent:

$$
\begin{gathered}
F_{b}=-m_{o} \omega^{2} r \\
m_{o}=m \bar{v} \rho
\end{gathered}
$$

where $m_{o}$ is the mass of the displaced solvent, $m$ the mass of the macromolecule, $\bar{v}$ the partial specific volume of the solute $(0.73 \mathrm{ml} / \mathrm{g}$ for most proteins $), \rho$ the density of the solvent.

The three forces come into balance very quickly, acceleration is lost, and hence, the macromolecule sediments with a constant velocity. The velocity of the macromolecule per unit gravitational acceleration is the sedimentation coefficient, $s$, defined in the Svedberg 
equation:

$$
\frac{M(1-\overline{\mathrm{v}} \rho)}{N f}=\frac{M D(1-\overline{\mathrm{v}} \rho)}{R T}=\frac{V}{\omega^{2} r}=s
$$

where $V$ is the radial velocity of the macromolecule, $R$ the gas constant, $T$ the absolute temperature, $D$ the diffusion coefficient. By inspecting the Svedberg equation, one may easily see the direct dependence of $s$ on the molar mass of the solute (which is corrected for the buoyancy in the equation) and inverse dependence on the frictional coefficient of the macromolecule. The sedimentation coefficient is expressed in Svedberg units (1 S = $\left.10^{-13} \mathrm{sec}\right)$.

\section{Sedimentation velocity AUC of proteins}

A sedimentation velocity (SV) AUC experiment primarily seeks to measure the sedimentation coefficient of a protein molecule. An absorbance scan at each time point during centrifugation yields a distribution of the concentration of species as a function of radial position in the cell (Fig. 10A). At the beginning of experiment, the concentration is uniform throughout the centrifugal cell. As the protein molecules sediment, they deplete from a meniscus region, and a moving boundary forms. The radial position of this boundary versus time (Fig. 10B) is the velocity of sedimentation, from which the sedimentation coefficient may be calculated using the Svedberg equation. It may be noted that the plateau concentration decreases over time due to radial dilution in sector-shaped cells. In addition, as the macromolecules sediment and concentration increases toward the bottom of the cell, the process of diffusion opposing the sedimentation intensifies, causing the boundary to spread as indicated by the decreasing incline of the concentration distribution curve. In principle, a higher angular velocity of the rotor would (and does in an SV experiment) 
A

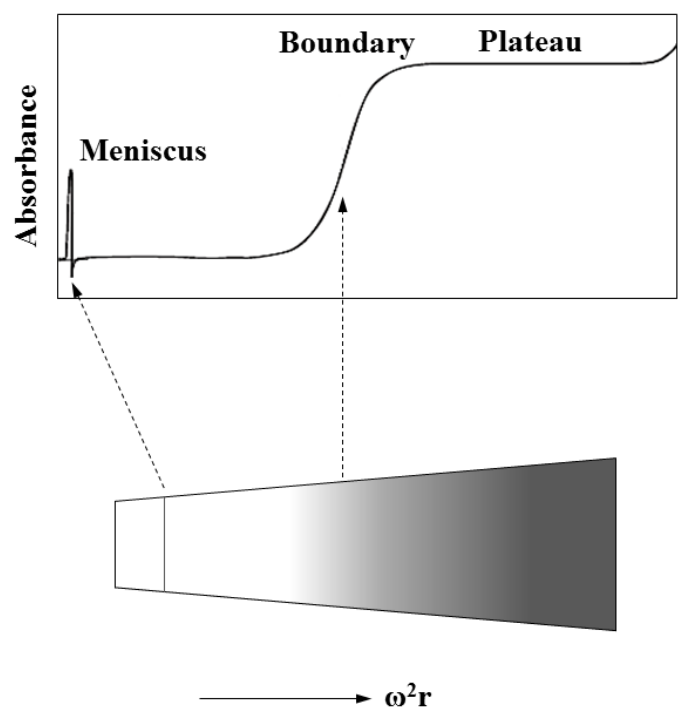

B

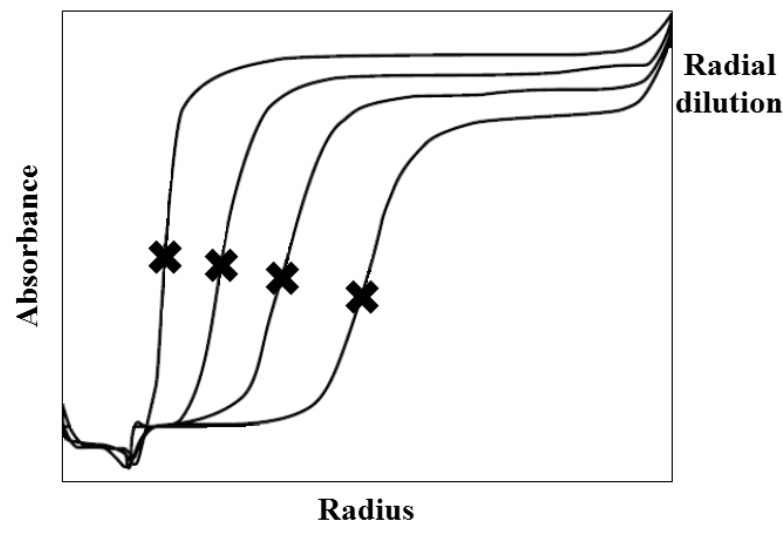

Fig. 10. Schematic of a sedimentation velocity AUC experiment. (A) Distribution of the analyte concentration during sedimentation at a single time point. (B) A series of absorbance scans taken at different time points represents moving boundary. $\mathbf{X}$ denotes boundary midpoint. The velocity of sedimentation is the radial position of this midpoint as function of time.

facilitate the sedimentation process resulting a sharper boundary and, ultimately, in better precision for the sedimentation coefficient measurement. However, valuable information concerning diffusion properties may be extracted from the rate of boundary spread. Thus, it is possible to calculate $s$ and $D$ (diffusion coefficient, measured in Ficks, $1 \mathrm{~F}=10^{-6}$ $\mathrm{cm}^{2} / \mathrm{sec}$ ) from a single SV experiment. These two values then can be used to determine the weight and frictional coefficient of the protein molecule. For comparison of the experimental values obtained under different conditions (buffer composition, temperature), $s$ and $D$ can be corrected to a standard state of water at $20^{\circ} \mathrm{C}$, indicated by a subscript 20,w:

$$
\begin{gathered}
s_{20, w}=s_{o b s} \times \frac{\left(1-\bar{v} \rho_{20, w}\right)}{\left(1-\bar{v} \rho_{T, b u f f e r}\right)} \times \frac{\eta_{T, \text { buffer }}}{\eta_{T, w}} \\
D_{20, w}=D_{o b s} \times \frac{\eta_{T, b u f f e r}}{\eta_{20, w}} \frac{293.1}{T}
\end{gathered}
$$


where $\eta$ is the viscosity of buffer and $T$ is the absolute temperature at which the experiment is conducted.

Such analysis of the moving boundary works well for a single highly homogeneous protein solute. It is also possible to analyze several sedimenting species, provided that their sedimentation properties differ enough to produce distinct boundaries. More often, the extent of those differences along with thermodynamic nonidealities do not allow observation of distinct boundaries. Modern computational analysis (reviewed in $(98,99)$ ), however, is capable of modeling experimental data points with the underlying transport equation (Lamm equation):

$$
\frac{d c}{d t}=\frac{1}{r} \frac{d}{d r}\left[r D \frac{d c}{d r}-s \omega^{2} r^{2} c\right]
$$

where $c$ is the weight concentration of the solute at radial distance $r$ at time point $t$.

The boundary spreading from differential migration and from diffusion is different, providing a basis for deconvolution of multiple sedimenting species. Direct boundary modeling, such as the $c(s)$-model in the Sedfit software developed by P. Schuck (Fig. 11), takes advantage of all experimental data points (often on the order of $10^{5}$ in a single experiment). For each $s$-value, a molar mass $\mathrm{M}(s)$ is calculated using partial specific volume $(0.73 \mathrm{ml} / \mathrm{g}$ for proteins $)$, the measured buffer density and viscosity, and an estimate for frictional coefficient $f / f_{0}$ as a fitting parameter. The $\mathrm{M}(s)$ and $s$ are then used to estimate the diffusion coefficient $D$. Finally, the $s$ and $D$ values are used to numerically solve the Lamm equation. A distribution of the Lamm equation solutions is then used to model the sedimentation boundary:

$$
a_{\text {measured }}(r, t)=\int c(s) L(s, D(s), r, t) d s+\operatorname{noise}(r, t)
$$




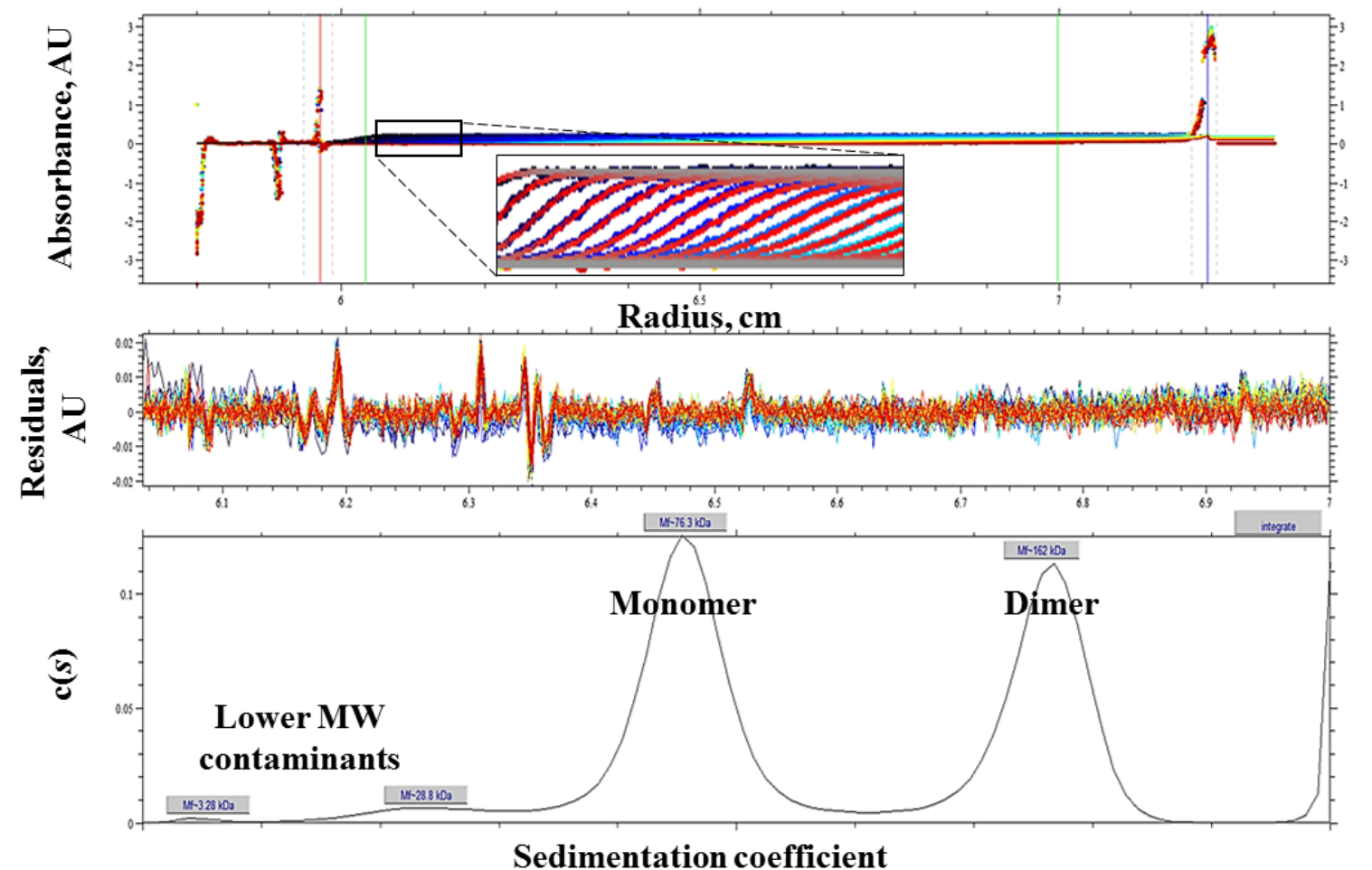

Fig. 11. Direct boundary modeling of sedimentation velocity data in the program Sedfit. Top panel depicts multiple absorbance scans (single points in inset) collected during the AUC experiment. The software models the sedimentation boundary (solid lines in the inset) using solutions of the Lamm equation. The quality of modeling can be assessed from the residuals (middle panel). Bottom panel depicts the distribution of sedimenting species as a function of sedimentation coefficient.

where $a$ is the measured optical signal as function of radial position and time, and $L(s, D(s), r, t)$ is the individual Lamm equation solution. The correct weight average $f / f_{0}$ is determined iteratively by nonlinear regression (the starting value is usually 1.2 , common for many globular proteins), thus further optimizing the fit. The resulting size distribution appears as a set of $\mathrm{c}(s)$ peaks, for each sedimenting species, scaled to reflect their relative loading concentrations. A peak $s$-value represents the weight average sedimentation coefficient, while the peak width is determined by the extent of $f / f_{0}$ deviation, thus reflecting diffusion properties of the species. The goodness of the fit is estimated by residuals of the fit. The $\mathrm{c}(s)$ model resolves well the sedimentation coefficients of monomer, dimer, etc. It is possible to convert $\mathrm{c}(s)$ into a $\mathrm{c}(M)$ distribution. The resulting estimate for the molar 
mass is usually within $5-10 \%$ of the correct value since this estimation is more sensitive to the $f / f_{0}$ factor than is the sedimentation coefficient.

\section{Sedimentation equilibrium AUC}

As the macromolecules sediment and concentrate at the bottom of the cell, the process of diffusion opposes the sedimentation (reviewed in (97)). While in an SV AUC experiment, the rotor speed (commonly 50,000 rpm) is adjusted so that sedimentation dominates diffusion, sedimentation equilibrium (SE) experiment is conducted at a lower rotor speed (usually $\leq 20,000 \mathrm{rpm}$ ). Given sufficient time, the two opposing processes approach equilibrium in all parts of the solution column, and at that point the concentration of solute increases exponentially towards the bottom. Such concentration gradient is a function of the molar mass:

$$
\ln C=\frac{\omega^{2} M(1-\bar{v} \rho) r^{2}}{2 R T}
$$

where $C$ is the concentration, $\omega$ the rotor angular velocity, $M$ the molar mass, $\bar{v}$ the analyte partial specific volume, $\rho$ the density of solvent, $r$ the radial position, $R$ the gas constant, $T$ the absolute temperature.

In a graphical representation, a plot of $\ln C$ versus $r^{2}$ takes a linear shape (Fig. 12) for a single species whose molar mass can be derived from the slope (or directly from the above equation). For multiple species, such as self-associating protein systems, the plot 'curves' upwards (Fig. 12B), and the slope of the tangent line at any point of that plot represents the average molar mass of the species with a corresponding radial position in a centrifugal cell at equilibrium. 
A

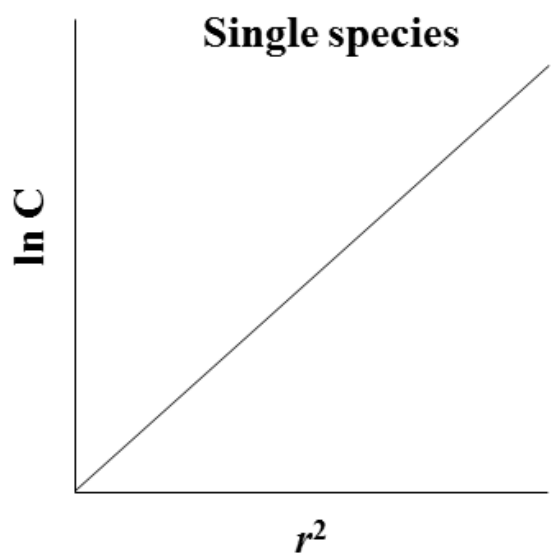

B

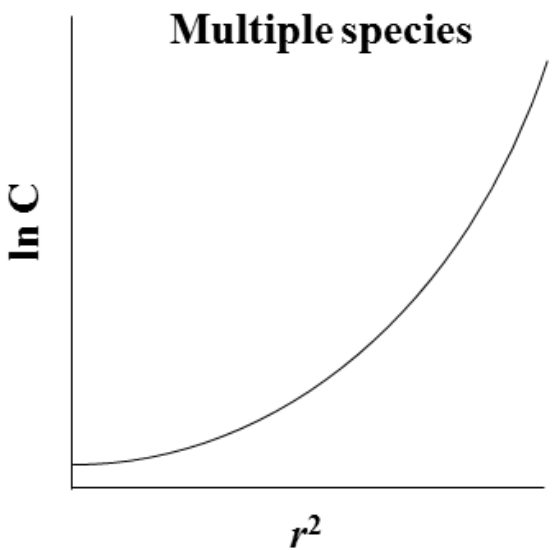

Fig. 12. Graphical analysis of sedimentation equilibrium data. Typical plots are presented for concentration distribution at equilibrium for single (A) and multiple (B) species.

In the case of a monomer-dimer equilibrium, at each point $r$ of the cell, the weight average molar mass of species $M_{w r}$ is defined:

$$
M_{w r}=\frac{C_{\text {monomer }} M+C_{\text {dimer }} 2 M}{C_{\text {monomer }}+C_{\text {dimer }}}=f_{\text {monomer }} M+f_{\text {dimer }} 2 M
$$

where $M$ is the molar mass of a monomer, $f$ is the fraction of monomer or dimer. Since $f_{\text {dimer }}=1-f_{M}$, the fraction of monomer can be expressed as

$$
f_{\text {monomer }}=2-\frac{M_{w r}}{M}
$$

Thus, the concentration of monomer and dimer at each point of the cell can be calculated along with the association constant $K_{\mathrm{a}}$ :

$$
K_{a}=\frac{C_{\text {dimer }}}{\left[C_{\text {monomer }}\right]^{2}}
$$

As with the SV experiments, modern software such as Sedphat (by P. Schuck) fits experimental data to an exponential model, and the measured signal as a function of radial position, $a(r)$ is defined: 


$$
a(r)=\sum_{n} c_{n, 0} \varepsilon_{n} d \exp \left[\frac{M_{n}\left(1-\bar{v}_{n} \rho\right) \omega^{2}}{2 R T}\left(r^{2}-r_{0}{ }^{2}\right)\right]+\delta
$$

(adapted from (98))

where the summation is over all species $n ; c_{n, 0}$ is the molar concentration of the species at a reference position $r o, \varepsilon$ is the molar extinction coefficient, $d$ is the optical path, $\delta$ is the baseline offset. As a result of the fit, weight average molar mass can be determined. For a self-associating system, the association constant is introduced, and the above equation takes the form:

$$
a(r)=\sum_{n} n \varepsilon_{1} d K_{n}\left(c_{1,0}\right)^{n} \exp \left[\frac{n M_{1}(1-\bar{v} \rho) \omega^{2}}{2 R T}\left(r^{2}-r_{0}{ }^{2}\right)\right]+\delta
$$

where the subscript ${ }_{1}$ denotes the monomer, and $K_{1}=1$ (adapted from (98)).

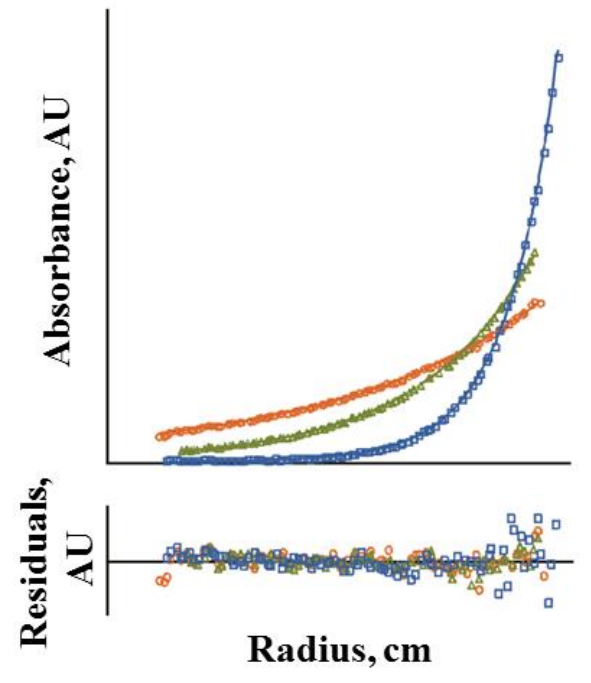

Fig. 13. Analysis of sedimentation equilibrium data obtained at different rotor speeds (different colors) in the program Sedphat. Single points represent experimental data, the lines represent theoretic fits (top panel). Bottom panel depicts residuals of the fit.
It is necessary to note that such analysis is very sensitive to noisy exponentials. For this reason, the analysis is typically performed as a global nonlinear regression fit of SE data obtained from a combination of different loading concentrations and rotor speeds (Fig. 13) to a selected model (monomerdimer equilibrium in this case). The goodness of the fit is often determined statistically using MonteCarlo noise simulations. 


\section{Scanning electron microscopy}

Scanning electron microscopy (SEM) is a technique for surface topography imaging. Over the past few decades, the SEM instrumentation has been significantly improved. Modern scanning electron microscopes are user-friendly and provide high resolution images at great magnifications with little aberrations. Being once an exclusive tool of material science, SEM has now become an attractive approach for ultrastructural examination of biological objects.

Ultrahigh resolution SEM imaging is achieved by utilizing a focused electron beam that is rastered from left to right and top to bottom to scan the specimen surface. The distribution of incident beam electrons in the specimen constitutes the interaction volume (Reviewed in (100), Fig. 14) whose spatial dimensions depend on specimen characteristics as well as the energy of the probe beam. Upon interaction with the beam, the specimen emits electrons, and based on the beam coordinates and the energy of emitted electrons, a rastered pattern of grayscale values (SEM image) is generated.

Interaction of the probe beam electrons (primary electrons) with the electric field of the atomic nuclei in a specimen results in elastic electron scattering, so called back scattering. The energy retained by the backscattered electrons is inversely proportional to the number of scattering events that primary electrons undergo before they 'escape' from the specimen and reach the detector, and ranges from $50 \mathrm{eV}$ to nearly incident beam energy. Interestingly, the degree of backscattered emission depends on the elemental composition of the specimen. Atoms with lower atomic number deflect electrons at smaller angles, and the electrons experience multiple elastic collisions and greater energy loss preceding their escape from the specimen. By contrast, higher atomic number nuclei deflect the electrons 
at larger angles, and fewer collision events are required to leave the specimen. The emitted backscattered electrons possess higher energies and can travel from relatively far below the surface before they

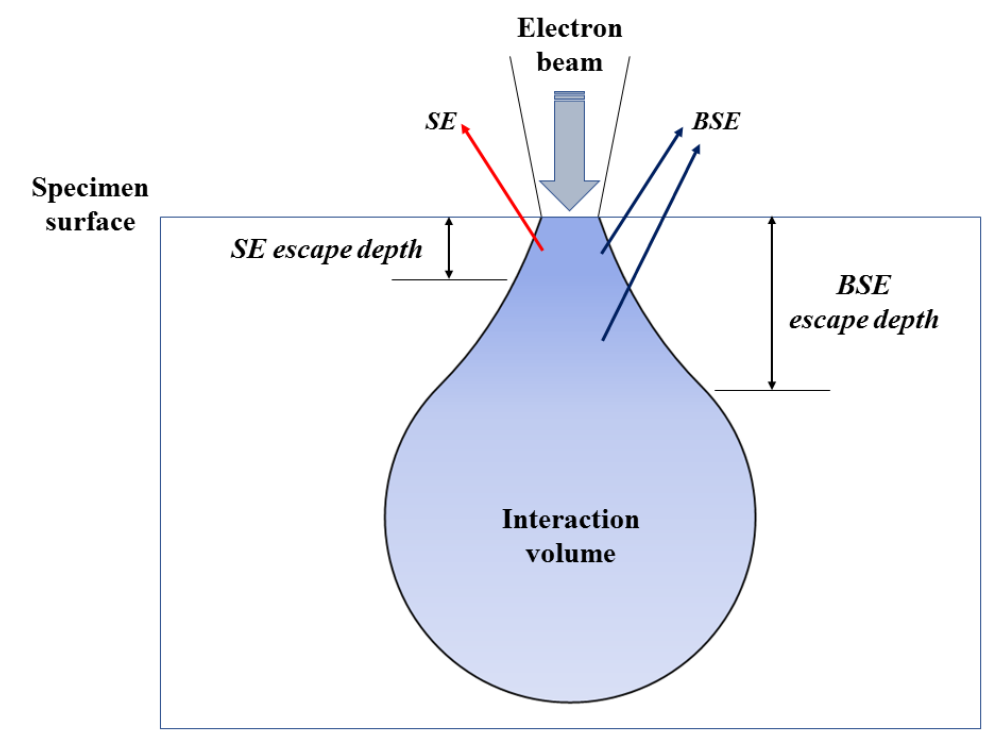

Fig. 14. Simplified representation of the interaction volume. BSE - backscattered electrons, SE - secondary electrons. Adapted from (100).

escape the specimen. In principle, the detection of emitted backscattered electrons can be used for specimen imaging. However, the linear dimensions of interaction volume at the escape depths of backscattered electrons (Fig. 14) are much wider than the cross-section of the probe beam. Such wider lateral dimension from which the signal can be generated results in lower scanning resolution. Thereby, this detection method is rarely used for topographic imaging. The depth of backscattering analysis is instead taken advantage of in examination of elemental distribution within the specimen.

Another type of interaction, inelastic collision, occurs when the primary beam electrons ionize specimen atoms causing them to expel secondary electrons. These secondary electrons possess low, $<50 \mathrm{eV}$ energies, and hence, can only be emitted from the very surface layer of a specimen. Electron scattering is negligible in that layer, and 
therefore secondary electron detection provides the most resolution for topographic imaging.

\section{Principal schematic of a scanning electron microscope}

Every SEM instrument has a beam generating system and a detection system. The electron beam forming system of an SEM instrument is typically regarded as a column (Fig. 15). The electron source (gun) is located at the top of that column. In a thermionic gun, free electrons are generated by emission from a tungsten filament at high (2700 K) temperature. A field-emission gun utilizes an effect of high electric field applied to a metal surface. The electron beam generated by a field-emission gun is much narrower and thus provides better imaging resolution than the thermionic beam. The electron beam from the gun passes between the condenser and objective 'lenses', which represent coil-wound electric wires that create an electron-repulsive magnetic field converging and accelerating the beam. Scanning coils mediate beam rastering by changing the intensity of electron repulsive field in different directions. Irradiation of the specimen with the beam causes emission of electrons. Those electrons hit a scintillator-coated detector thus generating light. The light is directed to a photomultiplier tube, where it is converted to an electrical signal. Electrons absorbed by the specimen are diverted to the ground by the metal specimen stage.

A controlled electron beam can only be created and function as a specimen probe under high vacuum conditions. Such conditions protect the gun filament from air oxidation and create a dust-free environment for proper function of the electron optics. In addition, the vacuum eliminates gas molecules and water vapors that would create an obstacle 
preventing electrons from reaching the specimen. Usually a system of two pumps (mechanical 'roughing' pump and turbo-molecular pump) maintains pressure at $10^{-6}-10^{-5}$ Torr inside the instrument.

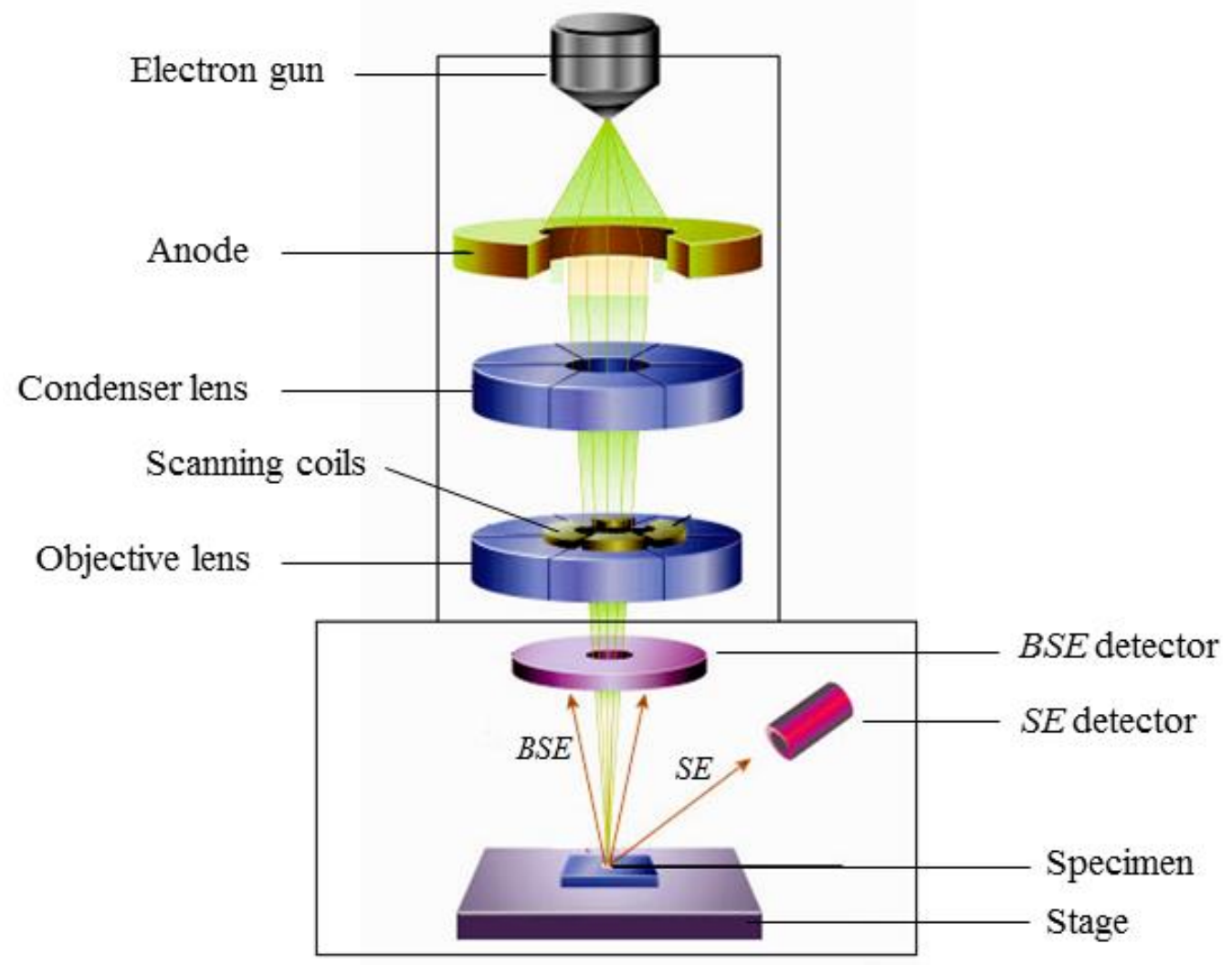

Fig. 15. Principal schematic of a scanning electron microscope. BSE - backscattered electrons, SE secondary electrons

\section{Special considerations in preparation of biological samples for SEM}

A major property shared by all biological specimens is the high water content. While low vacuum variable pressure SEM systems are available to study hydrated samples with little to no desiccation, they provide relatively low resolution thus limiting magnification at which imaging can be performed. In order to resolve the ultrastructural topography of a biological specimen, this specimen must be an electron conductive dry solid (reviewed in (101)). The main consideration taken in preparation of the biological 
SEM sample is the preservation of its structure as faithfully as possible to the native state. In particular, drying of the biological samples subjects them to a tensile stress resulting in inward shrinking and collapse. To avoid such structural damage, samples undergo fixation prior to drying. The fixation is usually achieved by crosslinking of proteins with glutaraldehyde, a homobifunctional reagent bridging two amines such as lysine side chains that are in close proximity. In addition, when necessary, osmium tetroxide is used for preservation of lipid structures.

Approaches to sample desiccation include freeze-drying, critical point drying, and air-drying. Freeze-drying is essentially a sublimation technique: the sample is immersed in liquid nitrogen followed by vacuum drying at low temperature. Such a 'one-step' technique is convenient for preparation of clean, debri-free samples. The two other methods require prior sample dehydration. This dehydration is commonly performed by stepwise immersion of the specimen into solutions with an increasing concentration of ethanol, accompanied by gradual dilution and ultimate elimination of water from the specimen. Although laborious, such passing of the sample through a series of solutions provides an additional washing benefit.

The principle behind critical point drying is that by increasing the pressure and the temperature of a wet sample, it is possible to achieve the critical point of the liquid at which the phase boundary between liquid and gaseous states is eliminated. The alcohol in the dehydrated sample is substituted for acetone, and then in a special apparatus, the acetone is exchanged with liquid $\mathrm{CO}_{2}$. The sharp temperature increase in the critical point drier causes transition of the liquid $\mathrm{CO}_{2}$ into the gas thus drying the sample.

For air-drying, the ethanol in the sample is replaced with hexamethyldisilazane 
(HMDS). HMDS is a highly volatile organic solvent that creates very little surface tension upon evaporation. Although HMDS is a highly toxic compound, the HMDS-drying does not require access to specialized equipment or liquid gases, and thus can be performed in a standard laboratory equipped with a ventilation cabinet.

The final stage in preparation of many biological objects for SEM analysis ensures their electron conductivity. As mentioned in the section above, the primary beam electrons absorbed by an electron-conductive specimen are diverted to the ground by a metal sample stage. Characterized by low electron conductivity, organic specimens are not able to transmit the absorbed electrons onto the stage. With ongoing electron irradiation, a negative charge is accumulated at the organic surface, causing deflection of the electron probe and resulting in image distortion and potentially in specimen damage. To address this issue, a thin conductive layer of metal is applied onto the sample using a sputter coater. Briefly, the sample is placed into an evacuated chamber, which is then filled with a carrier gas such as argon at a very low pressure. A high voltage is applied to ionize the carrier gas, and the resulting plasma ejects target material (e.g. a gold/palladium mixture) and sputters a thin metal alloy on the sample. Other metals used for coating include platinum, silver, chromium, iridium, and osmium. Depending on the sample dimensions and adhesive properties, application of additional conductive materials, such as sticky carbon or copper tape, may be necessary for more efficient coupling of the sample with the stage. 


\section{CHAPTER 3}

\section{RECOMBINANT EXPRESSION OF FXIIIA IN ESCHERICHIA COLI AND SITE-DIRECTED MUTAGENESIS}

\section{Introduction}

Protein research requires a substantial quantity of material. Although a number of proteins are commercially available, they do not always come at a low price. Furthermore, characterization of a protein often involves site-directed amino acid substitutions, extensive at times, for better understanding the structure and function relationship of that protein. For these, and multiple other reasons, a researcher greatly benefits from independent, in-house production of the protein of interest.

Early biochemical studies were performed using FXIII purified from plasma and platelets. The plasma FXIII $\mathrm{A}_{2} \mathrm{~B}_{2}$ concentration is $14-28 \mathrm{mg} / \mathrm{L}$ (corresponding to $86-172$ $\mathrm{nM}$ of the A-subunits) while platelets contain $46-82$ fg of FXIIIA 2 per platelet (given the mean platelet volume of $9 \mathrm{fL}, 61-110 \mu \mathrm{M}$ FXIIIA) (102-104). There is no doubt that purification of human FXIII demanded enormous amounts of source material and was a laborious process, with inevitably decreasing yields of the target protein after each purification step. A $25 \%$ prevalence of the FXIIIA V34L polymorphism, mentioned in the introductory chapter, only added to the complexity of biochemical characterization of blood-derived FXIII.

In 1990, Bishop and coworkers reported expression and purification of human 
FXIIIA from Saccharomyces cerevisiae (105). The amount of FXIIIA obtained upon cytosolic yeast expression was about $50 \mathrm{mg}$ per $\mathrm{L}$ of culture. For ease of purification, the Bishop group ligated a signal sequence to the FXIIIA gene that would promote FXIIIA secretion by the yeast cells into the medium. The attempt was unsuccessful, and higher yield was achieved instead by optimizing the fermentation conditions. During the final phase of fermentation, the level of FXIIIA reached approximately $2 \%$ of the total soluble protein, which was lower than that typically observed for other heterologous proteins, presumably due to toxicity of FXIIIA to the yeast cells (105). Nevertheless, the quantity of purified protein was sufficient to crystallize FXIIIA for X-ray studies (106).

The high-yield fermentation requires specialized equipment of limited availability and often at a commercial scale. Not all experimental techniques depend on as high a quantity of the protein material as to warrant acquisition of such equipment. In 1994, Greenberg and coworkers reported cloning and expression of FXIII A-chains in E. coli and their purification (107). In their initial attempts, predominantly a $30 \mathrm{kDa}$ fragment was produced, and very little full length FXIIIA. It was concluded from $\mathrm{N}$-terminal protein sequencing that the fragment resulted from internal translation starting at M474 or M475. Consistent with these data, a Shine-Dalgarno DNA sequence AGGA was found just upstream of the M474 codon. Converting the AGGA to TGGT DNA resulted in increase to the full length A-chain production without alterations to the primary protein sequence. In addition, the Greenberg group was able to express a series of FXIIIA mutants and characterize the functional importance of certain amino acid residues as well as structural domains (108-111).

In 2011, Smith and coworkers further optimized small-scale E. coli production of 
FXIIIA by cloning its DNA sequence into a pGEX plasmid (112). This expression vector contained an ampicillin resistance marker and encoded FXIIIA fused with N-terminal glutathione-S-transferase (GST) tag for affinity purification. The vector was later used for FXIIIA expression in the Maurer research group.

In addition to expression of WT FXIIIA, a number of experiments described in the subsequent chapters required introduction of amino acid substitutions into FXIIIA. In particular, residue 34 (naturally occurring valine or leucine) in the FXIIIA activation peptide has been shown to affect rates of FXIIIA activation by thrombin. Kinetic studies employing synthetic activation peptide fragments with different amino acid residues X34 (90-96) provided valuable information on how these substitutions are accommodated by thrombin and predicted that FXIIIA V34X substitutions may have different effects on the fibrin clot architecture. To validate this prediction experimentally, full length FXIIIA V34X variants ${ }^{1}$ were required to examine the effect of those substitutions on the fibrin clot architecture.

\footnotetext{
${ }^{1}$ All available amino acid sequences of mammalian FXIIIA contain leucine at position 34. The predominant human variant is V34, thus it is considered a wild type. Found in approximately $25 \%$ of Caucasian population, L34 FXIIIA has been routinely referred to as a "V34L polymorphism". Since the presence of L34 FXIIIA is not an isolated case, and no evidence has been reported for L34 being a result of mutation in human F13A1 gene, the V34L designation is rather misleading. While V34X designation occasionally appears in the present dissertation, it relates to FXIIIA variants obtained using site-directed mutagenesis of the template expression vector encoding V34 FXIIIA.
} 


\section{Materials and Methods}

\section{Materials}

Bacteriological grade tryptone was purchased from VWR. Bacto Yeast Extract was purchased from BD Biosciences Advanced Bioprocessing (Miami, FL). The QuikChange II Site-directed mutagenesis kit was purchased from Stratagene. The PreScission protease (PSP) was from GE Healthcare. At later stages of the current research project, an expression construct encoding GST-tagged PSP was generously provided by Dr. Jeffrey Keillor (University of Ottawa, Canada), and this protease was produced in-house. A fluorescence-based DNA Quantification Kit was from BioRad. The Miniprep DNA extraction kit was from QIAGEN. Bovine liver glutamate dehydrogenase and NADH were from Roche. All other reagents were from Sigma-Aldrich (now MilliporeSigma).

Oligonucleotide DNA primers were synthesized by IDT (Coralville, IA). A $\beta$ casein derived K9 peptide $\left({ }^{1}\right.$ LGPGQSKVIG ${ }^{10}$ ) was synthesized by New England Peptide (Gardner, MA), stock solutions (15-30 mM) were made in deionized $\mathrm{H}_{2} \mathrm{O}$, and accurate concentration was determined by quantitative amino acid analysis (AAA Service Laboratory, Damascus, OR).

Site-directed mutagenesis for obtaining protein expression constructs

A pGEX-6P-1 vector encoding FXIIIA was generously provided by Dr. Kerrie Smith, Dr. Robert Ariëns, and Dr. Helen Philippou (University of Leeds, UK). In this expression construct, FXIIIA DNA is inserted downstream of the GST-tag sequence, using BamH I as $5^{\prime}$ and Sal I as $3^{\prime}$ restriction sites. The GST is separated from FXIIIA by a sequence encoding the PSP recognition site (LEVLFQ $\downarrow$ GP) for cleavage of the expressed 
GST-FXIIIA fusion protein. pGEX-6P-1 also encodes $\beta$-lactamase conferring resistance to ampicillin and related antibiotics (Fig. 16).

The DNA mutagenesis was performed using the QuikChange Site-Directed Mutagenesis kit following the manufacturer's instructions. 20-50 ng of the template DNA was combined with the mutagenic primers (forward and reverse, 120-150 ng each, Table 1) in a $0.2 \mathrm{~mL}$ PCR tube containing reaction buffer, dNTP mix, and the Pfu Turbo DNA polymerase. The mutagenesis reaction was performed in sixteen thermal cycles (30 sec at $95^{\circ} \mathrm{C} ; 1 \mathrm{~min}$ at $55^{\circ} \mathrm{C} ; 7 \mathrm{~min}$ at $68^{\circ} \mathrm{C}$ ). Upon completion thermal cycling, the PCR samples were briefly chilled, supplemented with the $D p n$ I restriction enzyme, and incubated for 1 h at $37^{\circ} \mathrm{C}$ to digest parent DNA. The PCR product was then used to transform E. coli XL1 Blue supercompetent cells. Resistance to ampicillin was used as a selection marker.

To obtain concentrated DNA samples, the XL-1 Blue cells were grown at $37^{\circ} \mathrm{C}$ from a single colony in $2-5 \mathrm{ml}$ of LB broth containing $100 \mu \mathrm{g} / \mathrm{mL}$ ampicillin. Following overnight incubation, the cells were harvested by centrifugation at $5000 \mathrm{~g}$, and the plasmid DNA was extracted using the Qiagen Miniprep Kit. DNA concentration was determined on the Nanodrop spectrophotometer (access to this instrument was kindly provided by Ms. Betty Nunn from the Department of Bioengineering, University of Louisville). DNA sequencing was performed by the University of Louisville DNA Core facility.

Upon confirmation of the desired DNA substitutions, the plasmid vectors were transformed into E. coli BL21-Gold (DE3) competent cells (Agilent Technologies), using ampicillin resistance as a selection marker. Cells were then grown as described above for the XL-1 Blue cells, supplemented with $25 \%$ (v/v) glycerol, and stored at $-80{ }^{\circ} \mathrm{C}$. 


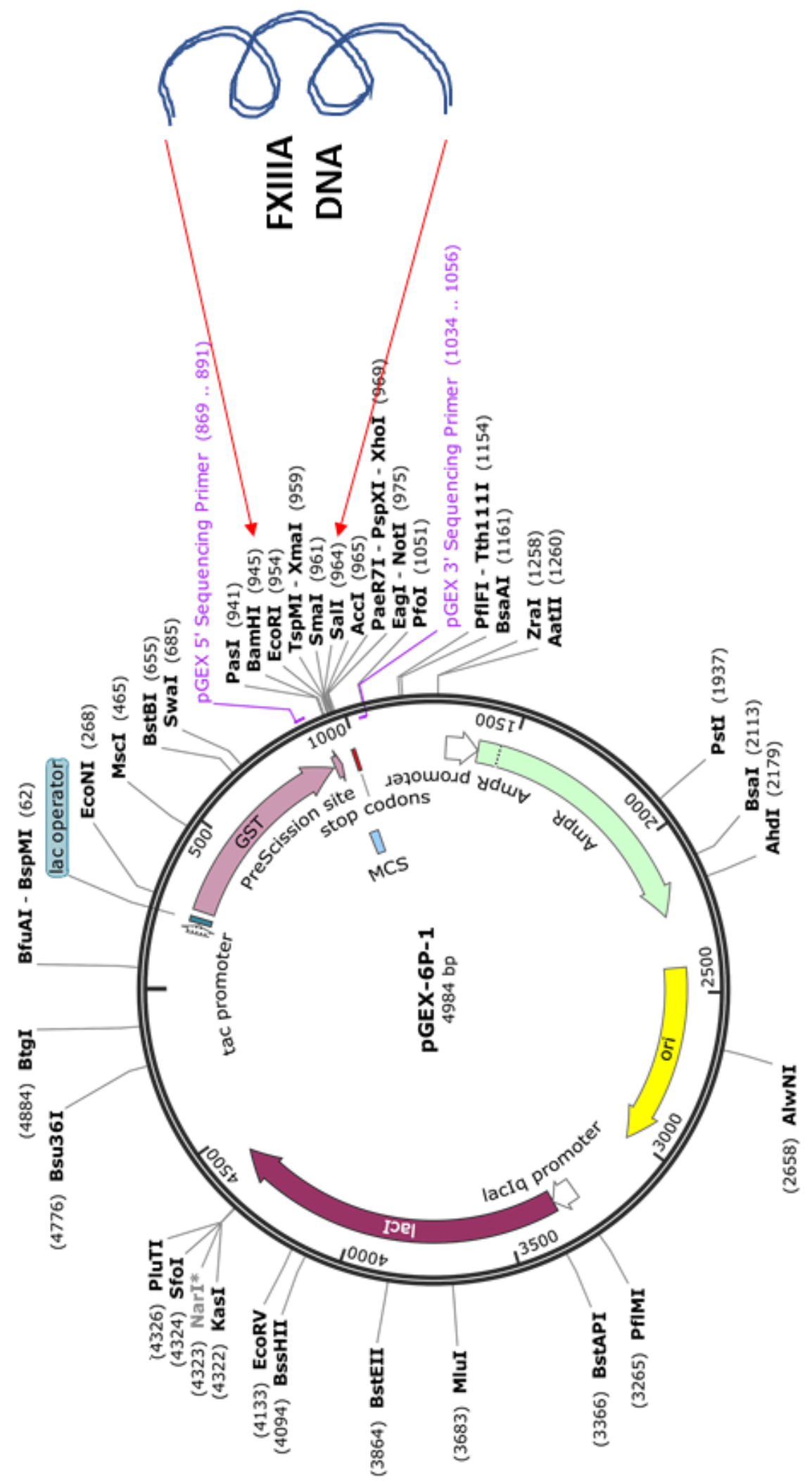

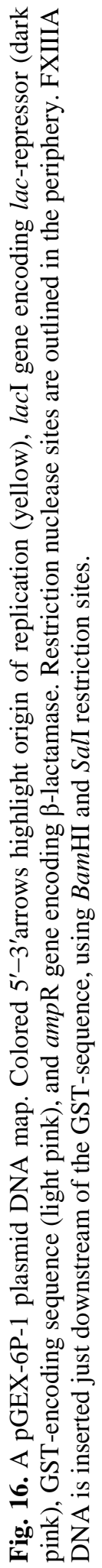


Table 1. Primers used for PCR-based site-directed mutagenesis to obtain expression vectors for FXIIIA variants.

\begin{tabular}{|c|c|}
\hline $\begin{array}{c}\text { FXIIIA } \\
\text { variant }\end{array}$ & $\begin{array}{c}\mathbf{5}^{\prime} \rightarrow \mathbf{3}^{\prime} \text { sequences } \\
\text { of forward and reverse mutagenic primers }\end{array}$ \\
\hline V34L & TTCAGGGCCTGGTGCCCC \\
& GGGGGCACAGGCCCTGAA \\
\hline V34F & GCTTCAGGGCTTCGTGCCCC \\
& GGGGGCAGAAGCCCTGAAGC \\
\hline \multirow{2}{*}{ V34W } & CTTCAGGGCTGGGTGCCCCGG \\
& CCGGGGCACCCAGCCCTGAAC \\
\hline
\end{tabular}

Recombinant protein expression Protocol I

TB medium (1.2\% Tryptone, $2.4 \%$ yeast extract, $0.4 \%$ glycerol, $100 \mu \mathrm{g} / \mathrm{ml}$ ampicillin; $200 \mathrm{~mL}$ ) was inoculated with E. coli BL21-Gold (DE3) cells from the freezer stock and grown overnight at $37^{\circ} \mathrm{C}$ in a rotary shaker at $225 \mathrm{rpm}$. This starter culture was then used to inoculate a fresh TB medium $(2 \mathrm{~L})$. The culture was grown at $37{ }^{\circ} \mathrm{C}$ with shaking $(225 \mathrm{rpm})$ until optical density at $600 \mathrm{~nm}\left(\mathrm{OD}_{600}\right)$ reached $0.8-1.0$. At this point, the protein expression was induced by addition of IPTG (to $1 \mathrm{mM}$ ) followed by $16 \mathrm{~h}$ incubation at $30^{\circ} \mathrm{C}$ with shaking $(180 \mathrm{rpm})$. Cells were harvested by centrifugation $\left(5000 \mathrm{~g}\right.$ ) at $4{ }^{\circ} \mathrm{C}$, rinsed to remove remaining medium, and the resulting pellets were stored at $-20{ }^{\circ} \mathrm{C}$.

\section{Recombinant protein expression Protocol II}

ZYP-0.8G non-inducing medium (113) (1\% tryptone, $0.5 \%$ yeast extract, $0.8 \%$ glucose, $50 \mathrm{mM} \mathrm{KH} 2 \mathrm{PO}_{4}, 50 \mathrm{mM} \mathrm{Na}_{2} \mathrm{HPO}_{4}, 25 \mathrm{mM}\left(\mathrm{NH}_{4}\right)_{2} \mathrm{SO}_{4}, 1 \mathrm{mM} \mathrm{MgSO}$, and 100 $\mu \mathrm{g} / \mathrm{mL}$ ampicillin; $5 \mathrm{~mL}$ ) was inoculated with the freezer stock cells and grown overnight at $37{ }^{\circ} \mathrm{C}$ with shaking $(225 \mathrm{rpm})$. The culture was then transferred into ZYP-5052 autoinducing medium ( $1 \%$ tryptone, $0.5 \%$ yeast extract, $0.5 \%$ glycerol, $0.05 \%$ glucose, 
$0.2 \% \alpha$-lactose, $50 \mathrm{mM} \mathrm{KH}_{2} \mathrm{PO}_{4}, 50 \mathrm{mM} \mathrm{Na} 2 \mathrm{HPO}_{4}, 25 \mathrm{mM}\left(\mathrm{NH}_{4}\right)_{2} \mathrm{SO}_{4}, 1 \mathrm{mM} \mathrm{MgSO}_{4}$, and $100 \mu \mathrm{g} / \mathrm{mL}$ ampicillin; $0.5-2.0 \mathrm{~L}$ ). The autoinducing culture was incubated at $37^{\circ} \mathrm{C}$ until the $\mathrm{OD}_{600}$ reached $0.5-1.0$, followed by the incubation at the room temperature for additional $16-24 \mathrm{~h}$. The agitation was maintained at $225 \mathrm{rpm}$ throughout the entire duration of cultivation. Biomass was harvested, rinsed, and stored as described in the section above.

\section{Recombinant protein extraction and purification}

Cell pellets were thawed and resuspended in the cold $\left(4{ }^{\circ} \mathrm{C}\right) 50-200 \mathrm{~mL}$ TBS $(50$ $\mathrm{mM}$ Tris Acetate $\mathrm{pH} 7.4,150 \mathrm{mM} \mathrm{NaCl}$ ). Cells were then lysed for $2 \mathrm{~h}$ at $4{ }^{\circ} \mathrm{C}$ in the presence of $1 \mathrm{mg} / \mathrm{mL}$ lysozyme, $1 \mathrm{mM}$ DTT, $2 \mu \mathrm{g} / \mathrm{mL}$ aprotinin, $1 \mu \mathrm{M}$ pepstatin $\mathrm{A}, 10 \mu \mathrm{M}$ leupeptin, $4 \mathrm{mM}$ benzamidine hydrochloride, $0.5 \mathrm{mM}$ PMSF, $0.05 \%$ sodium deoxycholate, $0.02 \%$ Triton X-100, and $5 \mu \mathrm{g} / \mathrm{mL}$ DNase I. Sometimes, RNase A was also added during the extraction process. The cell lysate was centrifuged at $22,000 \mathrm{~g}$ at $4{ }^{\circ} \mathrm{C}$, followed by treatment with $1 \%(\mathrm{w} / \mathrm{v})$ streptomycin sulfate to precipitate nucleic acid and additional centrifugation. After sterilization by vacuum filtration through a $0.2 \mu \mathrm{m}$ membrane filter, the clarified lysate was applied onto a $1 \mathrm{~mL}$ GST-trap affinity column using an Akta Prime FPLC system. The column was then excessively washed with TBS until the absorbance of eluent at $280 \mathrm{~nm}$ reached a baseline. The target protein was cleaved off the GST tag by oncolumn digestion with 100-200 $\mu \mathrm{g}$ PSP for $18 \mathrm{~h}$ at $4{ }^{\circ} \mathrm{C}$ and eluted with TBS. The GST was then eluted off the column with TBS containing $20 \mathrm{mM}$ reduced glutathione. FXIIIA molar concentration was determined from absorbance readings at $280 \mathrm{~nm}$ using $\varepsilon=125710 \mathrm{M}^{-1} \mathrm{~cm}^{-1}$ for $\mathrm{V} 34, \mathrm{~L} 34$, and F34 variants. An $\varepsilon=131210 \mathrm{M}^{-1} \mathrm{~cm}^{-1}$ was applied for FXIIIA W34. These extinction coefficients were calculated from the FXIIIA amino 
acid composition using the Protparam tool (www.expasy.org). The purity of preparations was assessed by SDS-PAGE. Protein fractions were stored at $-80^{\circ} \mathrm{C}$ in small $(50-200 \mu \mathrm{L})$ aliquots. Some protein samples were tested for DNA contamination with a Bio Rad DNA quantification kit following manufacturer instructions.

Activity of the recombinantly expressed FXIIIA AP variants

The transglutaminase activity of FXIIIA activation peptide (AP) variants was detected using a standard coupled spectrophotometric assay $(85,114,115)$. FXIIIA AP variant (final assay concentration $1 \mu \mathrm{M}$ ) was incubated for 5 min at $37{ }^{\circ} \mathrm{C}$ in $475 \mu \mathrm{L}$ of TBS in the presence of chromogenic assay components $(6.5 \mathrm{mM}$ glycine ethyl ester, 17 $\mathrm{mM} \alpha$-ketoglutaric acid, $3 \mathrm{mM} \mathrm{CaCl} 2,1.2 \mathrm{mM}$ ADP, $220 \mu \mathrm{M} \mathrm{NADH}, 12 \mathrm{U} / \mathrm{mL}$ glutamate dehydrogenase, $14 \mathrm{mg} / \mathrm{mL}$ BSA) and $30 \mathrm{nM}$ recombinant human thrombin. The reaction was initiated by addition of $25 \mu \mathrm{L}$ of $10 \mathrm{mM}$ glutamine donor peptide K9. FXIIIA expressed in S. cerevisiae served as a positive activity control.

\section{Results}

\section{Site-directed mutagenesis}

The results of initial site-directed mutagenesis attempts on the FXIIIA PGEX plasmid suggested an overall low output of the process. The efficiency of the XL-1 Blue cells transformation with the mutagenesis PCR product was not a concern, since multiple bacterial colonies would grow on the agar plates in the presence of ampicillin. However, most of the resulting plasmid samples contained original DNA, and on average, only one out of four sequenced clones carried a desired DNA substitution. Such outcome suggested 
that the amount of template DNA in PCR reaction was too high. The results were slightly improved by doubling the amount of Dpn I, the restriction nuclease that cleaves the methylated parent DNA. The best efficiency of the mutagenesis, however, was achieved by reducing the template plasmid from 50 to $20 \mathrm{ng}$ and increasing the mutagenic primers from 120 to $150 \mathrm{ng}$ in reaction. These amounts provided the optimal primer to template DNA ratio, and all isolated plasmid samples exclusively contained mutated DNA, even while using the standard manufacturer-suggested quantity of the Dpn I. As a result, the expression constructs were obtained for FXIIIA variants L34, F34, and W34.

\section{Optimization of recombinant protein expression}

Initially, the GST-tagged V34 FXIIIA was expressed in E. coli using a protocol obtained from the University of Leeds collaborators (Protocol I). This method was previously suitable for expression of fibrinogen $\alpha \mathrm{C}$ fragment (233-425). Briefly, BL21Gold (DE3) cells were transformed with a pGEX plasmid encoding GST-FXIIIA fusion protein. Protein expression was induced with IPTG, and the resulting fusion protein was affinity-purified on a GST-trap column. FXIIIA was freed from the GST tag by digestion with PSP and eluted off the column separately from GST.

The absorbance profile of the eluted FXIIIA protein was characterized by a distinct peak at $260 \mathrm{~nm}$ (Fig. 17A), thus suggesting a contamination with nucleic acids. By contrast, the GST fraction had the $280 \mathrm{~nm}$ maximum absorbance characteristic of pure protein (data not shown). The presence of DNA in protein preparations was unexpected, since the purification process included use of DNase and also streptomycin to precipitate the DNA. Given the absorbance of 1.4 at $260 \mathrm{~nm}$ and an average DNA extinction coefficient of 
$0.02 \mu \mathrm{g}^{-1} \mathrm{~mL}^{-1}$ at this wavelength, the DNA would be present at approximately $70 \mu \mathrm{g} / \mathrm{mL}$ in the sample. FXIIIA protein preparation was tested using a DNA quantification kit, and
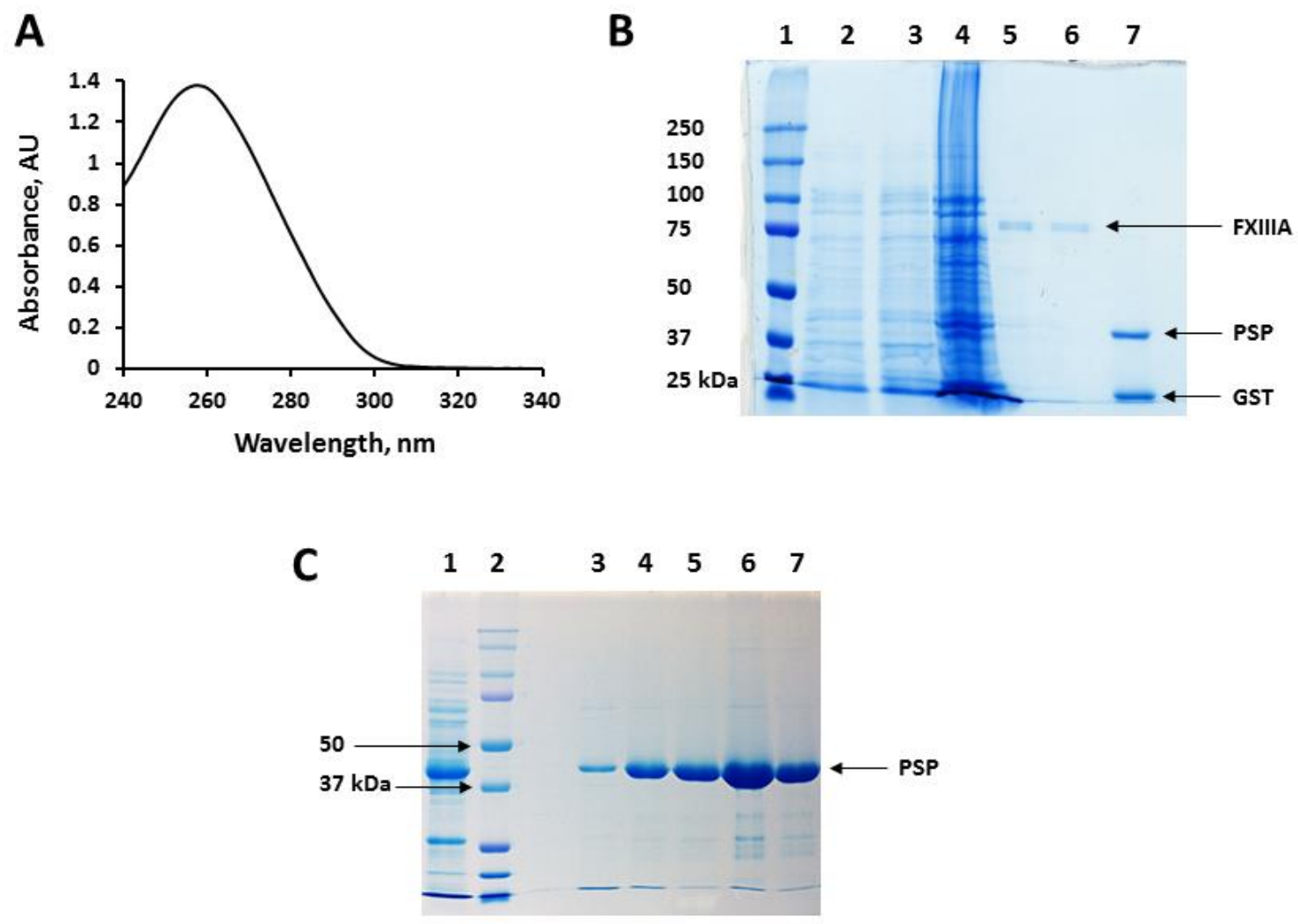

Fig. 17. Purification of FXIIIA (4 L culture) and PSP (500 mL culture) recombinantly expressed using Protocol I. (A) An absorbance scan of FXIIIA protein fraction demonstrating a $260 \mathrm{~nm}$ peak. (B) Assessment of FXIIIA protein preparations using 8\% SDS-PAGE: lane 1 - molecular weight marker, lanes 2 and 3 bacterial lysates prior (2) and post (3) IPTG induction. The loading amount in lane 3 was adjusted to account for differences in optical density of the bacterial cultures before and after IPTG induction. Lane 4 - clarified bacterial lysate, lanes 5 and 6 - FXIIIA-containing eluted fractions, lane 7 - 'GST'-fraction eluted with glutathione. (C) Assessment of PSP protein preparations using 10\% SDS-PAGE: lane 1 - clarified lysate, lane 2-molecular weight marker, lanes 3-7 - eluted PSP fractions.

the DNA content was below the detection limit of $10 \mathrm{ng} / \mathrm{mL}$. Thus, it was likely that the contaminating nucleic acid was RNA (a much appreciated proposal from Dr. Eugene Mueller, University of Louisville).

The FXIIIA protein bands appeared with the expected molecular weight of $\sim 83$ 
$\mathrm{kDa}$, however, they were barely detectable on the SDS-PAGE (Fig. 17B, lanes 5 and 6). On the contrary, the thickness of the GST band on the gels suggested higher levels of target protein expression (Fig. 17B, lane 7). Since no proteolytic FXIIIA fragments were detected on the SDS-PAGE (and no putative PSP cleavage sites were found within the FXIIIA amino acid sequence), it was concluded that FXIIIA may have precipitated upon cleavage of the GST-tag, and only a small fraction was able to elute off the column. Greenberg and coworkers were able to optimize a tag-free FXIIIA expression in E. coli by lowering IPTG concentration and incubation temperature (107); however, this strategy did not improve expression results in the current work. Notably, the PSP could readily be expressed in the Maurer lab using IPTG induction, yielding on average $12 \mathrm{mg}$ of protein from $1 \mathrm{~L}$ bacterial culture (Fig. 17C).

Following advice from Dr. Jeffrey Keillor (University of Ottawa, Canada), a different approach was implemented that involves induction of the protein expression by $\alpha$-lactose (Protocol II). As seen in Fig. 18A, the resulting FXIIIA protein had the expected protein absorbance profile with the characteristic peak at $280 \mathrm{~nm}$. The bands of the purified FXIIIA on the gel (Fig. 18B, lanes 4 and 5) indicated $>80 \%$ homogeneity and were much thicker that those observed after initial expression attempts using IPTG, and this time, were of comparable intensity with the GST bands (lanes 6 and 7). The yield of FXIIIA AP variants was on average $0.4 \mathrm{mg}$ per $1 \mathrm{~L}$ of the autoinducing bacterial culture.

This autoinduction protocol was also implemented for expression of the PSP (Fig. 17C, approximately $40 \mathrm{mg}$ yield per $1 \mathrm{~L}$ of culture). Compared to IPTG-induction, the autoinduction method resulted in four-fold increase of the PSP protein yield. This yield is underestimated since, as can be seen in Fig. 18C, the PSP amount in the cell lysate was so 

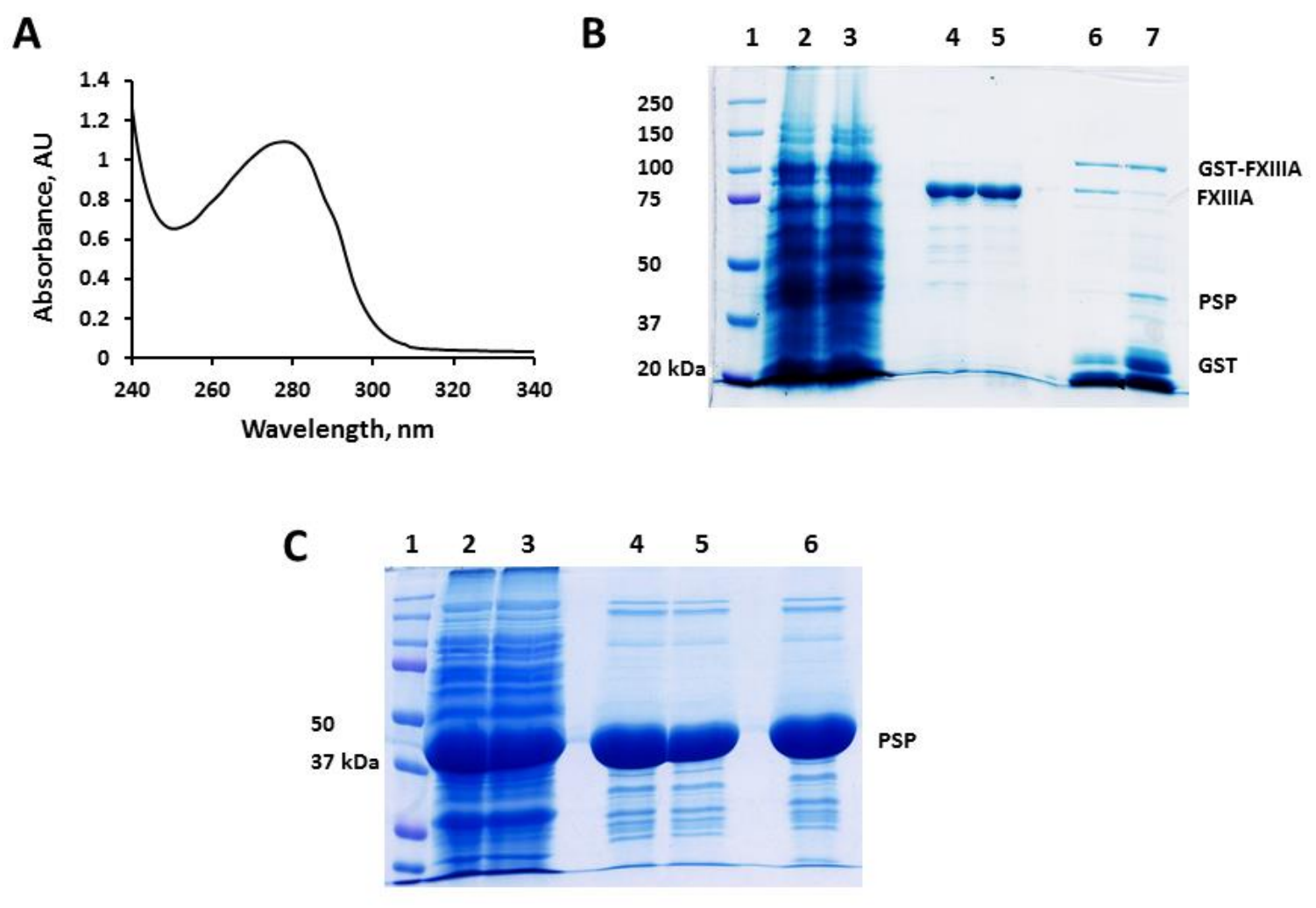

Fig. 17. Purification of FXIIIA (4 L culture) and PSP ( $250 \mathrm{~mL}$ culture) recombinantly expressed using Protocol II. (A) An absorbance scan of FXIIIA protein fraction demonstrating a $280 \mathrm{~nm}$ peak. (B) Assessment of FXIIIA protein preparations using 8\% SDS-PAGE: lane 1 - molecular weight marker, lanes 2 - clarified lysate, lane 3 - column flow-through upon lysate load, lanes 4 and 5 - FXIIIA fractions, lanes 6 and 7 glutathione-eluted fractions. (C) Assessment of PSP protein preparations using 10\% SDS-PAGE: lane $1-$ molecular weight marker, lane 2 - clarified lysate, lane 3 - column flow-through upon lysate load, lanes 4-6 - PSP fractions.

high that it exceeded the binding capacity of a $1 \mathrm{~mL}$ GST-trap column employed for purification, and this excess protein was not retained on the column (lane 3). The observed minor impurities would not create obstacles, since the PSP preparation was used in considerable $(\sim 1: 20)$ dilution for the current research. 


\section{Verification of activity of FXIIIA AP variants}

Unlike eukaryotic expression hosts, the intracellular environment of the E. coli strain used in this work is reducing. Such a difference could result in altered folding and diminished activity of the eukaryotic protein FXIIIA recombinantly expressed in E. coli. Besides, after cleavage of the GST-FXIIIA fusion protein by PSP, the resulting FXIIIA 'extra' N-terminal residues GPLGSAAM. Although the presence of those residues was not expected to affect FXIIIA conformation, the transglutaminase activity of FXIIIA variants expressed in E. coli had to be verified. FXIIIA activity was studied using a coupled ammonia release assay. Briefly, during the transamidation reaction FXIIIA incorporates a primary amine glycine ethyl ester (GEE) into a glutamine-containing K9-peptide with the release of ammonia. Glutamate dehydrogenase utilizes this ammonia along with NADH to convert $\alpha$-ketoglutarate to glutamate, accompanied by a sigmoidal decrease in absorbance at $340 \mathrm{~nm}$ (Fig. 19A).

Since the thrombin-mediated cleavage rates are different for the FXIIIA AP variants, a difference in generated activity would be expected. However, as seen in Fig. 19B, FXIIIA AP variants demonstrated comparable activity levels. The basis for such observation is not fully understood. As a possible explanation, in the coupled assay, the steep decrease of NADH is preceded by a lag-phase during which ammonia accumulates to the level necessary to saturate glutamate dehydrogenase. The presence of this lag-phase, along with ongoing activation by thrombin, could mask the effect of the different APcleavage rates. More importantly, activity of the E. coli-expressed FXIIIA was comparable to that of the control, yeast-expressed protein, thus indicating no significant perturbations to the native FXIIIA structure. 
A

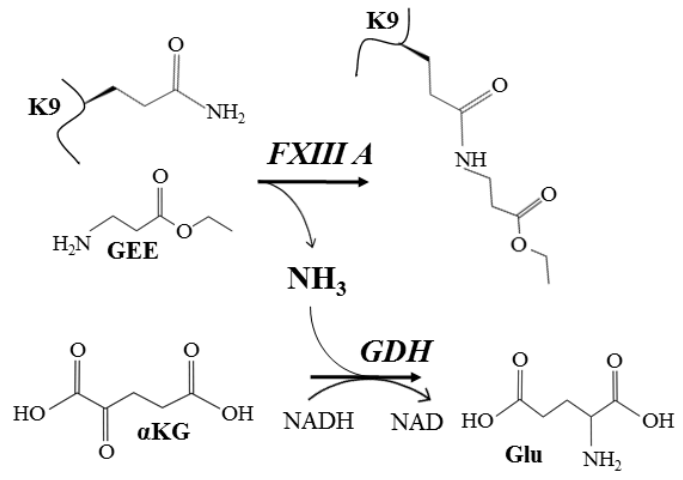

B

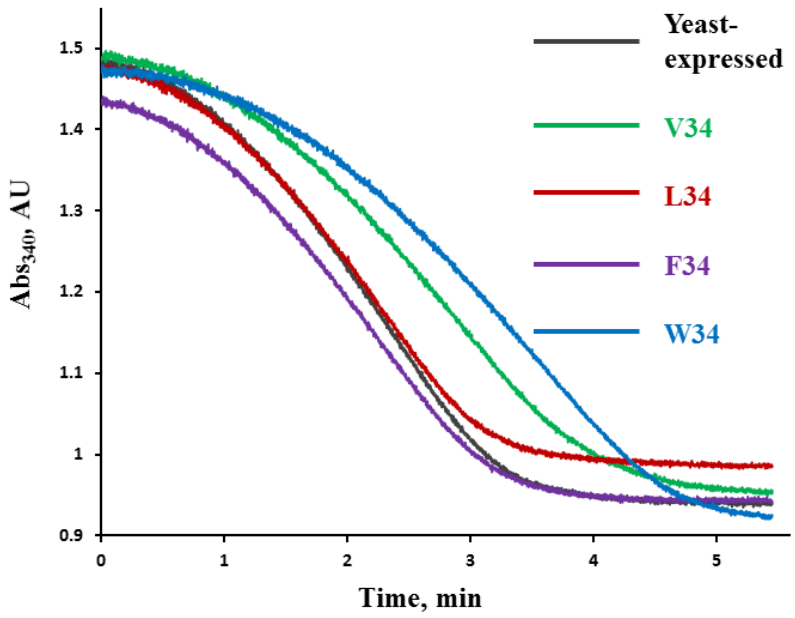

Fig. 19. Coupled transglutaminase activity assay. (A) A schematic of the assay. GEE - glycine ethyl ester, $\alpha \mathrm{KG}-\alpha$-ketoglutarate, Glu - glutamate. (B) Activity of recombinantly expressed FXIIIA AP variants. Activity of yeast-expressed FXIIIA is provided for comparison.

\section{Discussion}

Recombinant protein expression and purification in the current project were first attempted on the WT (V34) FXIIIA. Protein synthesis in E. coli with IPTG-induction proved not to be optimal for the expression of FXIIIA and resulted in very low protein contaminated with nucleic acid. To date, multiple researchers have reported complications in FXIIIA expression using IPTG induction $(107,113,116)$. In some cases, such as described by the Greenberg group, lowering IPTG concentration resulted in overall increased FXIIIA production in E. coli. Implementation of a similar approach, however, did not improve FXIIIA expression in the present study, likely due to the use of a different bacterial expression strain.

The E. coli host strain BL21-Gold (DE3) provides high recombinant protein expression levels. The lysogenized DE3 in this strain carries the gene of T7 RNA polymerase under the control of an inducible lacUV5 promoter. Upon addition of the inducer, the lacUV5 promoter is derepressed, and the T7 RNA polymerase is expressed. 
This RNA polymerase then transcribes target protein DNA from the plasmid expression vector. Even low basal generation of the T7 RNA polymerase can result in expression of some target proteins without induction. To avoid such unintended expression, the target gene in the pGEX plasmid is under control of the tac promoter.

Being derivatives of the E. coli lac promoter, both lacUV5 and tac promotors direct mRNA synthesis upon induction with the lactose analog IPTG. Enhanced inducibility of these promotors provides high levels of the target DNA transcription, which is ultimately supposed to result in high protein expression levels. However, tryptone, a major component of the bacterial growth media, is often contaminated with lactose $(113,116)$. Even trace amounts of lactose in the medium may induce the lac-derived promotors. Aggravated by the absence of a natural catabolite repressor such as glucose in standard media, the DNA transcription and protein synthesis are initiated at early stages of cultivation, when the temperature is maintained relatively high. The fast buildup of polypeptide at such high temperature often results in a failure of orthologous proteins to adopt native conformation, and this issue may be especially relevant to the current research. As mentioned in Chapter 1, FXIIIA has a particular four-domain fold. In addition, there are three cis-peptide bonds present in the FXIIIA molecule, and the energetically unfavorable cis-isomerization has been reported to be a rate limiting step in protein folding $(117,118)$. Adding to the complexity, such misfolding may have resulted in an impaired ability of FXIIIA to form a correct dimer interface.

Besides the desperately low yields, a prominent feature of FXIIIA preparations obtained using the IPTG induction protocol employed in this work was nucleic acid contamination. Since DNA could not be detected in the preparations of FXIIIA, it was the 
RNA that may have co-purified with the target protein, but no published reports describe the specific interaction of FXIIIA with RNA. However, given exhaustive washing steps (over 50 column volumes) during FXIIIA purification process, a specific RNA binding to the GST-FXIIIA fusion protein immobilized on the affinity column remained a threatening possibility.

Experimental investigation of RNA binding to the GST-FXIIIA fusion protein is beyond the scope of the current project, and instead the following speculation may be offered. It is well known that unfolded proteins bind to the 50S subunit of the prokaryotic ribosome, and in a recent report, Pathak et al. (119) demonstrated that the presence of an unfolded protein in above stoichiometric amounts, promotes dissociation of ribosome subunits, thereby reducing the fold-assisting function of the ribosome. As suggested above, at the relatively high incubation temperature, the FXIIIA polypeptide is assembled faster than it folds into the native conformation. This unfolded FXIIIA may thus have caused the dissociation of E. coli ribosomes. Such dissociated ribosomes are susceptible to degradation by cellular ribonucleases (119). Whether the actual ribosome degradation has or has not taken place in the initial 'IPTG expression' efforts, it is possible that some ribosomal RNA remained bound with the fusion protein on the column despite excessive washing steps. Two current research observations indicate RNA association with the FXIIIA portion of that protein. First, nucleic acid contamination was detected in the samples of FXIIIA cleaved off the GST tag, and no such contamination was observed in the GST-containing fractions. Second, two other GST-tagged proteins (PSP and fibrinogen $\alpha \mathrm{C}(233-425))$ could readily be expressed in the Maurer lab using conventional IPTG induction protocol, and both purified proteins were consistently nucleic acid-free. 
Instead of IPTG, the alternative autoinduction approach $(113,116)$ to protein expression relies on the use of a natural inducer of the lac promotor, $\alpha$-lactose, in conjunction with a catabolite repressor, glucose, in the bacterial growth media. As a preferable carbohydrate substrate, glucose prevents transport of lactose into the cells and hence, limits premature induction of the lac-related promoters, thus allowing the bacterial culture to reach a certain degree of growth without overexpressing the target protein. Once glucose is depleted from the medium, the cells switch to $\alpha$-lactose utilization, synchronously inducing target protein synthesis. Lowering the temperature below $30{ }^{\circ} \mathrm{C}$ at this stage allows the target protein to fold 'correctly', ultimately increasing the yield of the preparation. Indeed, implementation of the autoinduction protocol immediately resulted in an increase of FXIIIA preparation quality.

Overall, a series of catalytically competent FXIIIA AP variants were expressed, and the success of the current expression protocol is attributed to a more efficient control of expression induction. That is, no induction is believed to occur in early stages of the bacterial growth. Increased agitation (225 versus $180 \mathrm{rpm}$ ) provides better aeration of the bacterial culture. In addition, lower temperature regime (room temperature $21-26^{\circ} \mathrm{C}$ versus $30{ }^{\circ} \mathrm{C}$ ) aids in slowing the synthesis of the target polypeptide allowing it to adopt a more native-like fold. 


\section{CHAPTER 4}

\section{PROBING OLIGOMERIC STATES OF FXIIIA IN SOLUTION}

\section{Introduction}

Plasma FXIII is found mostly as a heterotetramer of two catalytic A-subunits, and two carrier B-subunits $\left(\mathrm{A}_{2} \mathrm{~B}_{2}\right)$, and $\sim 1 \%$ as an $\mathrm{A}_{2}$-homodimer (120). The intracellular form, for example in platelets, is observed exclusively as $A_{2}$ (3). Both plasma and cellular FXIII oligomers are stabilized by non-covalent inter-subunit interactions.

The A-subunit of FXIII (FXIIIA) is a transglutaminase (protein glutamine:amine $\gamma$-glutamyltransferase) that is expressed as an inactive zymogen form (1). Two different activation paths are currently considered for plasma- and intracellular FXIII. In a final stage of blood coagulation, thrombin cleaves the $\mathrm{N}$-terminal $4 \mathrm{kDa}$ activation peptides on the $\mathrm{A}$ subunits of plasma FXIII $\mathrm{A}_{2} \mathrm{~B}_{2}$. Then in the presence of low $\mathrm{mM} \mathrm{Ca}^{2+}$, the B-subunits dissociate from the A-subunits exposing catalytically active transglutaminase (61). In contrast, intracellular FXIIIA 2 is thought to undergo non-proteolytic activation by $\mathrm{Ca}^{2+}$ (62-64). In vitro, FXIIIA 2 can be activated by thrombin in essentially the same manner as plasma FXIII $\mathrm{A}_{2} \mathrm{~B}_{2}$, but without involvement of the B-subunits. In addition, the intracellular nonproteolytic activation has been routinely simulated by the presence of nonphysiologically high $(>50 \mathrm{mM}) \mathrm{Ca}^{2+}(82) . \mathrm{Ca}^{2+}$ has been demonstrated to be crucial for transglutaminase activity, whereas other divalent cations are much less effective 
$\left(\mathrm{Ca}^{2+}>\mathrm{Sr}^{2+}>\mathrm{Ba}^{2+}>\mathrm{Mg}^{2+}\right)$ (65). Furthermore, the binding of $\mathrm{Ca}^{2+}$ stabilizes FXIIIA preventing its degradation by proteases (121).

Although both active sites in FXIIIA 2 are exposed upon activation by thrombin, previous inhibition studies $(14,73,82)$ revealed that only one mole of radiolabeled iodoacetamide (IAA) was incorporated per two moles of thrombin-activated A-subunits (half-of-the-sites reactivity), thus negative cooperativity was proposed for the catalytic action of FXIIIA. Interestingly, no large differences between the zymogen and thrombinactivated forms were observed in early X-ray crystal studies $(69,122)$. However, with a FXIIIA-catalyzed reaction where two polypeptide chains are cross-linked, significant rearrangements in the enzyme would be required to bind substrate and to release the product. Indeed, solution-based hydrogen-deuterium exchange and chemical modification experiments indicated conformational alterations with opening of the FXIIIA dimer interface upon activation (84-86). Consistent with those conclusions, non-proteolytically activated FXIIIA that had been covalently inhibited was later crystallized with an exposed active site and proposed to be monomeric (72). In the present work, the oligomeric state of the A-subunits of FXIII is addressed in a solution environment.

Single amino acid substitutions in FXIIIA are believed to have the potential to disrupt dimer formation. A few candidates had been identified in patients with severe FXIII deficiency: R260L, R260C, Y283C, and G562R. Activation peptide substitution R11Q was computationally predicted to impair $\mathrm{A}_{2}$ dimer formation. Most of those mutants were unstable in eukaryotic expression systems (74-79). Recombinant expression in E. coli along with affinity purification could be more feasible for obtaining mutant proteins, however, this approach had not been attempted before. 


\section{Materials and Methods}

\section{Materials}

Recombinant FXIIIA expressed in S. cerevisiae was a generous gift from the late Dr. Paul Bishop (Zymogenetics, Seattle, WA). A stock solution of FXIIIA (40 $\mu$ M) was made in deionized water. Concentration was determined from the absorbance at $280 \mathrm{~nm}$ using $\varepsilon=125710 \mathrm{M}^{-1} \mathrm{~cm}^{-1}$ (calculated using Protparam tool, www.expasy.org), and aliquots were stored at $-80^{\circ} \mathrm{C}$. Molar concentration of FXIIIA in this work refers to the A-subunits, as opposed to $\mathrm{A}_{2}$-dimers. Bovine thrombin was purchased from SigmaAldrich. Thrombin inhibitor d-phenylalanyl-prolyl-arginyl chloromethyl ketone (PPACK) was purchased from Haematologic Technologies (Essex Junction, VT). FXIIIA inhibitor peptide K9-DON (85) was synthesized by Zedira (Darmstadt, Germany), and a stock solution $(500 \mu \mathrm{M})$ was made in dimethyl sulfoxide (DMSO). All other reagents were from Sigma-Aldrich.

\section{FXIIIA sample preparation for AUC}

All samples were prepared in a final volume of $500 \mu \mathrm{L}$ for sedimentation velocity and $130 \mu \mathrm{L}$ for sedimentation equilibrium experiments. To study nonproteolytic activation of FXIIIA, 2-6 $\mu \mathrm{M}$ zymogen was incubated in borate buffer $(20 \mathrm{mM}$ boric acid, $150 \mathrm{mM}$ $\mathrm{NaCl}, \mathrm{pH} 7.8$ ) in the presence of $100 \mathrm{mM} \mathrm{CaCl}_{2}$ at $37^{\circ} \mathrm{C}$ for $30 \mathrm{~min}$. As a negative control for a divalent metal cation, $100 \mathrm{mM} \mathrm{MgCl}_{2}$ was employed. For proteolytic activation, FXIIIA $(2-6 \mu \mathrm{M})$ was treated with $3.5 \mathrm{NIH} \mathrm{U} / \mathrm{mL}$ bovine thrombin in the borate buffer containing $4 \mathrm{mM} \mathrm{CaCl} 2$ for $30 \mathrm{~min}$ at $37^{\circ} \mathrm{C}$, following by addition of $760 \mathrm{nM}$ thrombin inhibitor PPACK. 
For inhibition studies, FXIIIA $(2 \mu \mathrm{M})$ activated by thrombin in the borate buffer with $4 \mathrm{mM} \mathrm{CaCl}_{2}$ was inhibited with five-fold molar excess of alkylating agent iodoacetamide (IAA) or five-fold molar excess of covalent inhibitor peptide K9-DON. Samples of inhibited FXIIIA were supplemented with 5\% DMSO.

Control samples of zymogen FXIIIA $(2 \mu \mathrm{M})$ were made in PBS (10 mM sodium phosphate, $150 \mathrm{mM} \mathrm{NaCl}, \mathrm{pH} 7.4(105))$ or in borate buffer.

$A U C$

The oligomerization and hydrodynamic properties of zymogen as well as activated FXIIIA were studied by analytical ultracentrifugation. Samples were subjected to centrifugation within $2 \mathrm{~h}$ of preparation.

Sedimentation velocity experiments were carried out in a Beckman Coulter ProteomeLab XL-A analytical ultracentrifuge at $20{ }^{\circ} \mathrm{C}$ and $50,000 \mathrm{rpm}$ in standard twosector cells. Buffer density was determined on a Mettler/Paar Calculating Density Meter DMA 55A at $20^{\circ} \mathrm{C}$ and viscosity was measured using an Anton Parr AMVn Automated Microviscosimeter at $20^{\circ} \mathrm{C}$. Data were analyzed with the program Sedfit (www.analyticalultracentrifugation.com) using the continuous $\mathrm{c}(s)$ distribution model. The partial specific volume of FXIIIA was calculated from the amino acid composition $(0.734 \mathrm{~mL} / \mathrm{g})$ using the Protparam tool (www.expasy.org). Frictional ratios were calculated by Sedfit assuming no bound water. Experimental sedimentation coefficients were converted to $s_{20, \mathrm{w}}$ using the corrections based on the measured density and viscosity. Analytical runs were performed with at least two independent samples for each condition examined. 
Sedimentation equilibrium experiments were carried out in standard two-sector cells using $150 \mu \mathrm{L}$ of buffer in the reference sector and $130 \mu \mathrm{L}$ of sample in the sample sector. Three different rotor speeds were used and equilibrium was confirmed by observing the identical distribution of $280 \mathrm{~nm}$ absorbance in three successive scans at $2 \mathrm{~h}$ intervals for each rotor speed. Data were analyzed using the program Sedphat. Global analysis was carried out using the monomer-dimer self-association model. Error analysis was performed using the Monte-Carlo error analysis feature of Sedphat with 1000 iterations.

Intrinsic tryptophan fluorescence measurements

Fluorescent spectra were collected using a Perkin Elmer LS55 spectrofluorimeter. FXIIIA samples were prepared in the same manner as for the AUC. Prior to the measurements, each sample (500 $\mu \mathrm{L})$ was transferred into a $4.0 \mathrm{ml}$ quartz cuvette and diluted with corresponding buffer to a final volume of $2.0 \mathrm{ml}$ (thus resulting in final FXIIIA concentration of $500 \mathrm{nM}$ ). Samples were excited at $280 \mathrm{~nm}$, and emission spectra at 300$400 \mathrm{~nm}$ were recorded. Fluorescence measurements were conducted with two independent samples for each condition studied.

Native polyacrylamide gel electrophoresis ( $n P A G E)$

Samples of FXIIIA were prepared similarly to the AUC studies but in smaller volumes (20-100 $\mu \mathrm{L})$. Samples were then supplemented with a loading buffer ( $0.5 \mathrm{M}$ Tris, $\mathrm{pH} 6.8 ; 20 \%$ glycerol, $0.01 \% \mathrm{w} / \mathrm{v}$ bromophenol blue) in a 2:1 sample:buffer ratio. Electrophoretic separation was conducted at room temperature in a Tris-Glycine running buffer without SDS using 4\% stacking and 7.5\% resolving gels. During electrophoresis, 
the temperature of the running buffer did not elevate above $28{ }^{\circ} \mathrm{C}$. Gels were stained with Coomassie Blue. At least three independent samples were studied for each condition, with essentially the same results.

Size exclusion chromatography (SEC)

SEC was conducted using a $10 \times 300 \mathrm{~mm}$ Superdex 75 analytical column (GE Healthcare) on an Akta FPLC (GE Healthcare). The column was calibrated with gel filtration standard from BioRad containing proteins of known Stokes radii $\left(R_{\mathrm{S}}\right)$ : bovine $\gamma$ globulin $\left(150 \mathrm{kDa}, R_{\mathrm{S}}=5.1 \mathrm{~nm}\right)$, chicken ovalbumin $\left(43 \mathrm{kDa}, R_{\mathrm{S}}=2.8 \mathrm{~nm}\right)$, and equine myoglobin $\left(17 \mathrm{kDa}, R_{\mathrm{S}}=1.9 \mathrm{~nm}\right)$. Dextran Blue and vitamin $\mathrm{B}_{12}$ were used to determine the void column volume $\left(V_{0}\right)$ and the total volume $\left(V_{\mathrm{t}}\right)$, respectively. The BioRad standard protein mixture was separated in borate buffer. Samples of zymogen FXIIIA as well as $100 \mathrm{mM} \mathrm{Ca}^{2+}$ and $100 \mathrm{mM} \mathrm{Mg}^{2+}$-treated FXIIIA were prepared as described for the sedimentation velocity AUC. Due to possible precipitation of the thrombin-activated FXIIIA on the analytical column, this form was not subjected to SEC analysis.

For each run, a $100 \mu \mathrm{L}$ aliquot of $2 \mu \mathrm{M}$ protein sample was applied to the column that had been pre-equilibrated with corresponding buffer. All protein separations were carried out at $0.5 \mathrm{ml} / \mathrm{min}$, room temperature. Protein elution was monitored by absorbance at $280 \mathrm{~nm}$. The peak elution volume $\left(V_{\mathrm{e}}\right)$ of each protein was converted to a partition coefficient $(\sigma)$ using the equation

$$
\sigma=\frac{\left(V_{e}-V_{0}\right)}{\left(V_{t}-V_{0}\right)}
$$

where $V_{\mathrm{t}}$ is the total column volume and $V_{0}$ is the void volume.

An inverse error function $\left(\mathrm{erf}^{-1}\right)$ was calculated for (1- $\left.\sigma\right)(123)$ of each calibration 
standard and plotted against their Stokes radii. This calibration "curve" was then used to determine $R_{\mathrm{S}}$ for different forms of FXIIIA.

Site-directed mutagenesis, recombinant protein expression and purification

Experimental manipulations for obtaining FXIIIA mutants with proposed impaired dimerization were conducted as described in Chapter 3. The primer pairs used for mutagenesis PCR are listed in the Table 2, along with brief descriptions of the mutants.

Table 2. Primers used for PCR-based site-directed mutagenesis to obtain expression vectors for FXIIIA mutants.

\begin{tabular}{|c|c|c|}
\hline $\begin{array}{l}\text { FXIIIA } \\
\text { mutant }\end{array}$ & Description & $\begin{array}{c}5^{\prime} \rightarrow 3^{\prime} \text { sequences } \\
\text { of forward and reverse } \\
\text { mutagenic primers }\end{array}$ \\
\hline R11Q & $\begin{array}{l}\text { The activation peptide residue R11 forms a salt } \\
\text { bridge with D343 of the opposite subunit. The } \\
\text { R11Q substitution was computationally } \\
\text { predicted to impair dimer formation, however, it } \\
\text { could not be expressed in eukaryotic cells (79). }\end{array}$ & $\begin{array}{l}\text { CTTTGGAGGCCAGAGAGCAGTTC } \\
\text { GAACTGCTCTCTGGCCTCCAAGG }\end{array}$ \\
\hline R260L & $\begin{array}{l}\text { R260 is located at the dimer interface. A } \\
\text { substitution may result in impaired } \\
\text { dimerization. R260L was expressed in COS- } \\
\text { cells and underwent rapid intracellular } \\
\text { degradation (74). }\end{array}$ & $\begin{array}{l}\text { CAAAGTCAGCCTGGTGGGGTC } \\
\text { GACCCCACCAGGCTGACTTTG }\end{array}$ \\
\hline R260C & $\begin{array}{l}\text { Molecular modeling of R260C predicted } \\
\text { impaired dimerization. Expression in BHK- } \\
\text { cells resulted in rapid degradation. R260C } \\
\text { expressed in yeast, subjected to gel filtration, } \\
\text { and was concluded to be a monomer ( } 75-77) \text {. }\end{array}$ & $\begin{array}{l}\text { CAAAGTCAGCTGCGTGGGGTC } \\
\text { GACCCCACGCAGCTGACTTTG }\end{array}$ \\
\hline Y283C & $\begin{array}{l}\text { Y283 is located in the catalytic core of FXIIIA. } \\
\text { Y283C was expressed in MEG-cells at very low } \\
\text { level. Gel filtration suggested monomeric state } \\
\text { of the mutant, and impaired binding with B- } \\
\text { subunits was observed (78). Remarkably, } \\
\text { residue Y283 is conserved among the } \\
\text { transglutaminase family with the majority of } \\
\text { members existing as monomers state. }\end{array}$ & $\begin{array}{l}\text { GGGACAATATCTGTGCCTATGGCGTC } \\
\text { GACGCCATAGGCACAGATATTGTCCC }\end{array}$ \\
\hline G562R & $\begin{array}{l}\text { G562 is located in } \beta \text {-barrel 1. G562R mutant } \\
\text { was expressed in MEG-cells, and was } \\
\text { concluded to exist as a monomer from gel } \\
\text { filtration analysis }(75,78) \text {. }\end{array}$ & $\begin{array}{l}\text { СTTCTACACCCGCGTCCCGAAGG } \\
\text { CCTTCGGGACGCGGGTGTAGAAG }\end{array}$ \\
\hline
\end{tabular}




\section{Results}

Activated FXIIIA species experience significant conformational rearrangement compared to the zymogen form

In an attempt to probe FXIIIA for possible conformational rearrangements in solution upon activation, a series of intrinsic tryptophan fluorescence measurements was performed (Fig. 20A). To activate FXIIIA proteolytically (by cleavage of the activation peptide), the zymogen was incubated with thrombin in the presence of $4 \mathrm{mM} \mathrm{Ca}^{2+}$. Nonproteolytic activation was achieved in the presence of $100 \mathrm{mM} \mathrm{Ca}^{2+}$. An overall fluorescence decrease (17-18\%) was observed for activated FXIIIA forms relative to the zymogen, suggesting an increase in polarity of the environment surrounding some of the tryptophan residues in the FXIIIA molecule. Thus, these tryptophan residues became more solvent-exposed, indicating conformational rearrangements in the $\mathrm{A}_{2}$-homodimer. As a control, FXIIIA was also treated with $100 \mathrm{mM} \mathrm{Mg}^{2+}$, and only a slight effect on intrinsic fluorescence of the protein (5\% decrease) was detected. While activation by thrombin was optimal at physiological temperature $\left(37^{\circ} \mathrm{C}\right)$, high $\mathrm{mM}$ Ca-activation proceeded even at room temperature $\left(21-26^{\circ} \mathrm{C}\right)$, and the fluorescence decrease could be monitored continuously in the non-thermostated instrument. Under conditions of both proteolytic and nonproteolytic activation, fluorescence reached minimum values at $30 \mathrm{~min}$.

To further assess possible dissociation of FXIIIA upon activation, a series of nondenaturing polyacrylamide gel electrophoresis (nPAGE) experiments was conducted. A significant change in FXIIIA electrophoretic mobility was visible upon activation by thrombin (Fig. 20B, lane 2) and by $100 \mathrm{mM} \mathrm{Ca}^{2+}$ (Fig. 20B, lane 3). However, these conditions caused the protein to spread along a migration path (especially in thrombin- 

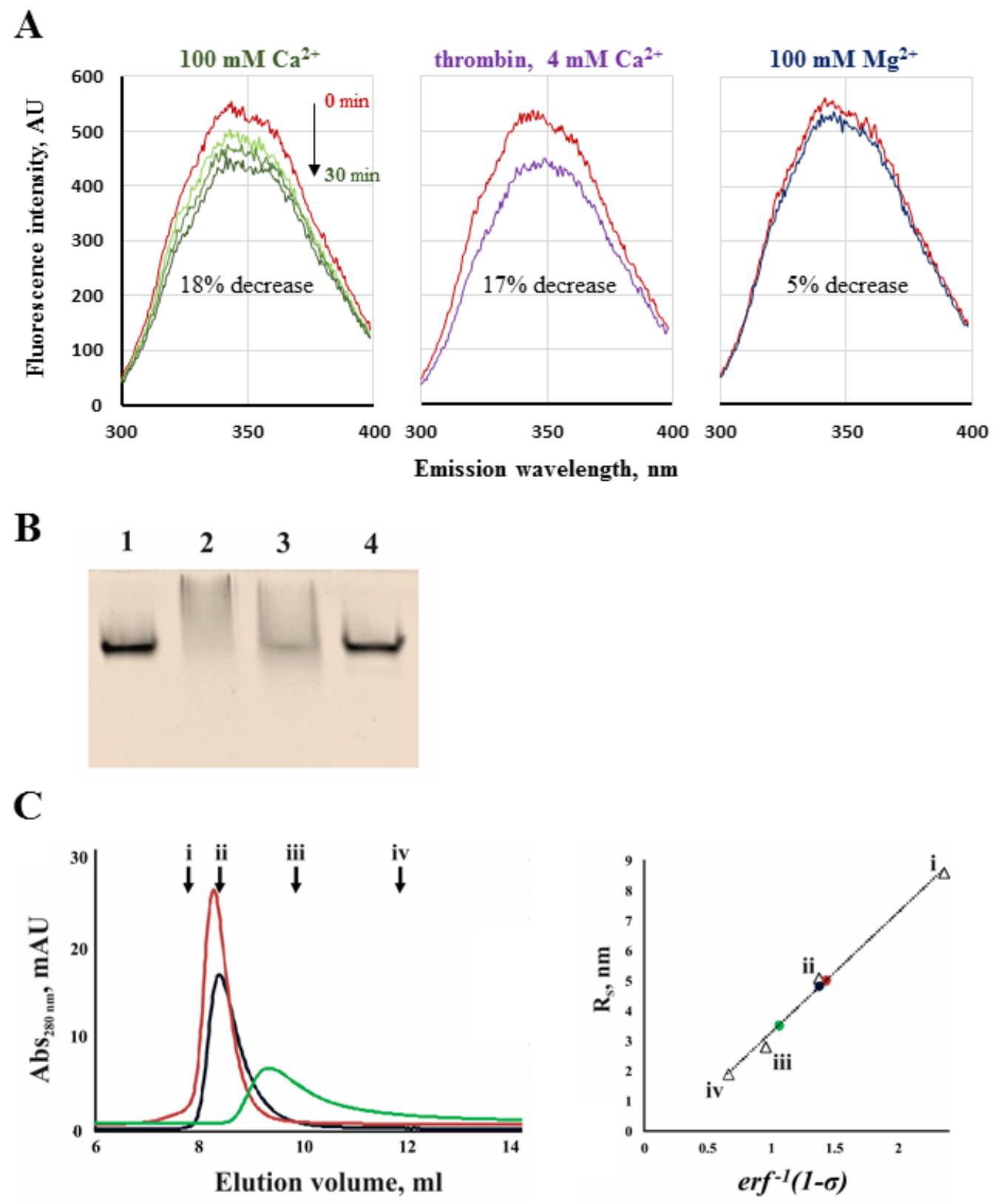

Fig. 20. (A) Fluorescence emission spectra of FXIIIA under different conditions. Control zymogen FXIIIA is shown in red; (B) nPAGE of FXIIIA: lane 1 - zymogen FXIIIA; lane 2 - FXIIIA activated by thrombin in the presence of $4 \mathrm{mM} \mathrm{CaCl}_{2}$; lane 3 - FXIIIA activated with $100 \mathrm{mM} \mathrm{CaCl}_{2}$; lane 4 - FXIII treated with $100 \mathrm{mM} \mathrm{MgCl} 2$; (C) SEC elution profiles of FXIIIA under different conditions (left): zymogen in borate buffer (red), FXIIIA in the presence of $100 \mathrm{mM} \mathrm{CaCl}_{2}$ (green), and FXIIIA in the presence of $100 \mathrm{mM}$ $\mathrm{MgCl}_{2}$ (dark blue). Arrows indicate peak elution volumes $\left(V_{\mathrm{e}}\right)$ of the calibration standards (i thyroglobulin, $670 \mathrm{kDa}, V_{\mathrm{e}}=7.80 \mathrm{ml}$; ii - bovine $\gamma$-globulin, $158 \mathrm{kDa}, V_{\mathrm{e}}=8.40 \mathrm{ml}$; iii - chicken ovalbumin, $43 \mathrm{kDa}, V_{\mathrm{e}}=9.87 \mathrm{ml}$; iv - equine myoglobin, $17 \mathrm{kDa}, V_{\mathrm{e}}=11.89 \mathrm{ml}$ ); calculation of $R_{\mathrm{S}}($ right) for FXIIIA forms based on their partition coefficients $(\sigma):$ erf $^{-1}(1-\sigma)$ values were plotted versus $R_{\mathrm{S}}$ for the calibration standards (i $-8.6 \mathrm{~nm}$, ii $-5.1 \mathrm{~nm}$, iii $-2.8 \mathrm{~nm}$, iv $-1.9 \mathrm{~nm}$ ). The $R_{\mathrm{S}}$-values were calculated for FXIIIA samples (circles): $5.0 \mathrm{~nm}$ for zymogen, $4.8 \mathrm{~nm}$ for FXIIIA treated with $100 \mathrm{mM} \mathrm{MgCl}_{2}$, and 3.5 $\mathrm{nm}$ for FXIIIA in the presence of $100 \mathrm{mM} \mathrm{CaCl}_{2}$. 
activated FXIIIA) yielding no well-defined bands on the gel. By contrast, the migration of FXIIIA treated with $100 \mathrm{mM} \mathrm{Mg}^{2+}$ (Fig. 20B, lane 4) was identical to that of the zymogen form (Fig. 20B, lane 1).

Finally, FXIIIA was analyzed by size exclusion chromatography (SEC, Fig. 20C). Both zymogen and $100 \mathrm{mM} \mathrm{Mg}^{2+}$-treated FXIIIA had similar elution profiles. Their Stokes radii were determined to be $5.0 \mathrm{~nm}$ and $4.8 \mathrm{~nm}$, respectively. In the presence of 100 $\mathrm{mM} \mathrm{Ca}{ }^{2+}$, FXIIIA eluted after the zymogen form, and its Stokes radius was $3.5 \mathrm{~nm}$. These observations were consistent with the possibility of dissociation of FXIII $\mathrm{A}_{2}$-homodimer upon non-proteolytic activation.

\section{FXIIIA $_{2}$ dissociates into monomers upon activation}

Although fluorescence measurements, nPAGE, and SEC results indicated significant perturbations in FXIIIA upon activation, the oligomerization states of the enzyme forms remained inconclusive. In contrast, analytical ultracentrifugation (AUC) provided distinct resolution of the FXIIIA species. Representative results of sedimentation velocity analyses are summarized in Table 3 and Fig. 21. The dimeric state of FXIIIA zymogen in solution was confirmed (Fig. 21, trace i). The sedimentation coefficient of $\sim 7.9 \mathrm{~S}$ was consistent with the molecular weight of $\sim 160 \mathrm{kDa}$. A frictional ratio of $\sim 1.3$ indicated a slightly asymmetric molecule. In the presence of $100 \mathrm{mM} \mathrm{Mg}{ }^{2+}, 90 \%$ of the protein existed in the dimeric form (Fig. 21, trace iii). A profound change in sedimentation properties was observed in the presence of $100 \mathrm{mM} \mathrm{Ca}^{2+}$ (Fig. 21, trace ii). This nonproteolytically activated FXIIIA migrated uniformly with a sedimentation coefficient of $\sim 4.8 \mathrm{~S}$, consistent with the molecular weight $\sim 80 \mathrm{kDa}$, thus indicating a monomeric state. 
Table 3. Hydrodynamic parameters of FXIIIA.

\begin{tabular}{|c|c|c|}
\hline Sample & s20,w, $\mathbf{S}$ & Frictional ratio \\
\hline FXIIIA zymogen & 7.91 & 1.32 \\
& 7.85 & 1.35 \\
\hline FXIIIA with $100 \mathrm{mM} \mathrm{Ca}^{2+}$ & 4.83 & 1.38 \\
& 4.80 & 1.31 \\
\hline FXIIIA with $100 \mathrm{mM} \mathrm{Mg}^{2+}$ & 7.93 & 1.35 \\
& 7.73 & 1.35 \\
\hline FXIIIA thrombin-activated in presence of $4 \mathrm{mM} \mathrm{Ca}^{2+}$ & 4.57 & 1.53 \\
& 4.70 & 1.37 \\
\hline
\end{tabular}

Sedimentation coefficients (in Svedberg units, S) and frictional ratios were obtained by analyzing sedimentation velocity data using the Sedfit software. Observed $s$-values were corrected to $s_{20, w}$. For each experimental condition, data are presented for two independently made samples.

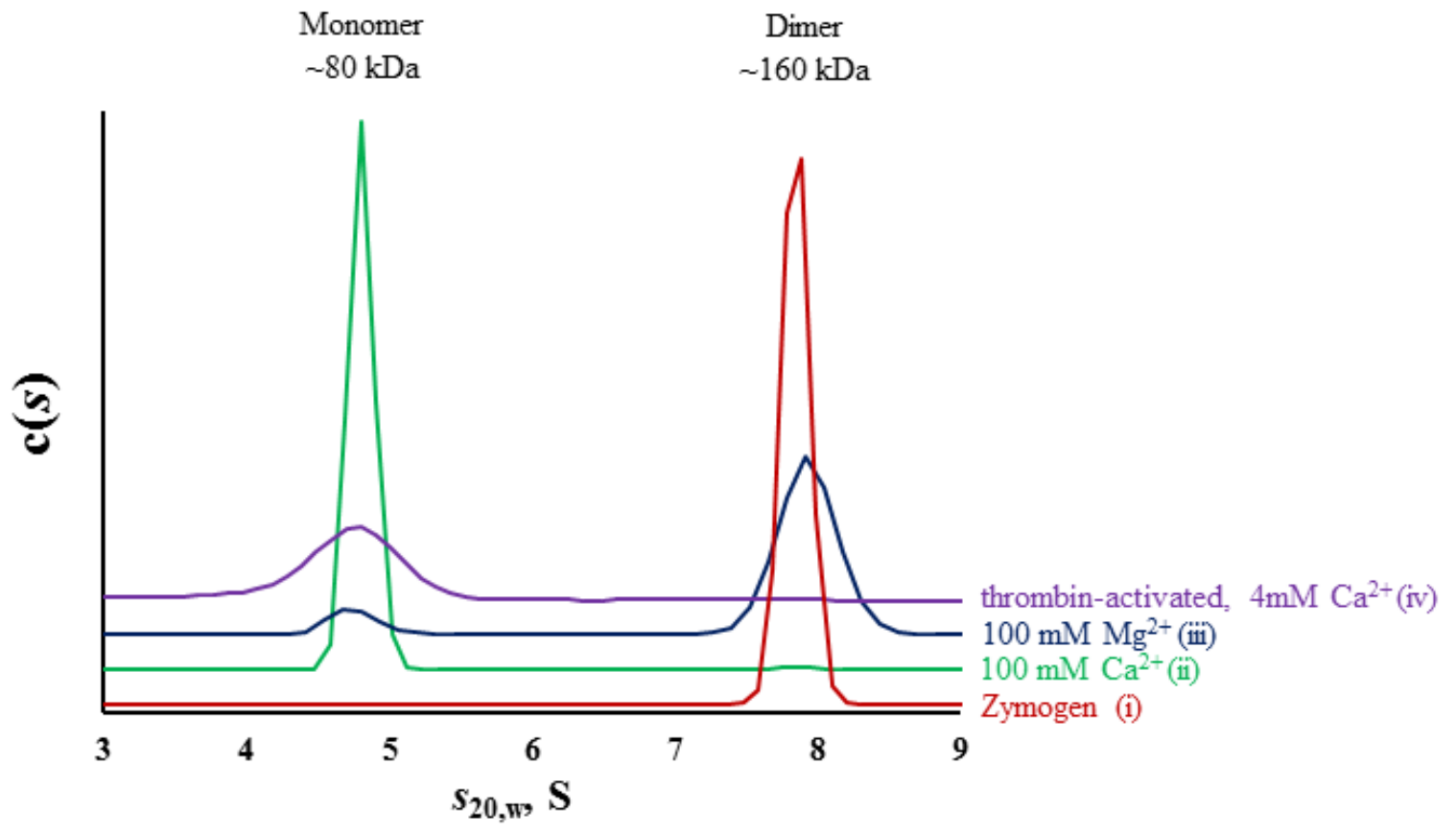

Fig. 21. Representative sedimentation velocity profiles of FXIIIA. (i) - FXIIIA zymogen in the borate buffer; (ii) - FXIIIA incubated in the presence of $100 \mathrm{mM} \mathrm{CaCl}_{2}$; (iii) - FXIIIA incubated in the presence of $100 \mathrm{mM} \mathrm{MgCl}_{2}$; (iv) - FXIIIA cleaved by thrombin in the presence of $4 \mathrm{mM} \mathrm{CaCl}_{2}$. For all conditions, loading concentration of FXIIIA was $2 \mu \mathrm{M}$. $\mathrm{c}(s)$ is the concentration distribution as a function of $\mathrm{s}_{20, \mathrm{w}}$. 
A more detailed analysis of the non-proteolytically activated FXIIIA oligomeric state is presented in Chapter 5.

AUC of thrombin-activated FXIIIA revealed a monomer with a sedimentation coefficient of $\sim 4.6 \mathrm{~S}$ (Fig. 21, trace iv). Although no significant differences could be seen between the frictional ratios of thrombin-cleaved FXIIIA compared to non-proteolytically activated enzyme (Table 3), the broader peak of the thrombin-activated form (Fig. 21, trace iv) was suggestive of a more flexible conformation of FXIIIA after activation peptide cleavage. In addition, as previously observed $(73,124)$, thrombin-activated FXIIIA tends to aggregate over the course of a few hours, causing its low abundance in solution and thus making it difficult to analyze by both SEC and AUC. Whereas non-denaturing treatment with sodium deoxycholate or dithiothreitol did not resolve this issue, the addition of 5\% DMSO was found to facilitate solubility of the active FXIIIA, without causing dissociation of zymogen FXIIIA $_{2}$ or affecting the enzyme catalytic activity (data not shown).

\section{Quantitative analysis of FXIII A-subunits interactions}

To assess inter-subunit interactions in FXIIIA quantitatively, a series of sedimentation equilibrium AUC experiments was conducted. Representative distribution profiles obtained for the zymogen and activated forms at three different rotor speeds are presented in Fig. 22. Global fitting of experimental data to a monomer-dimer equilibrium model yielded apparent dissociation constants $\left(K_{\mathrm{D}}\right)$ for dimerization of FXIIIA. As expected, interactions of the A-subunits in the zymogen form were relatively tight $\left(K_{D}=8\right.$ $\mathrm{nM})$. Much higher $K_{\mathrm{D}}$-values (indicating weaker interactions) were calculated for activated FXIIIA: $90 \mathrm{mM}$ for non-proteolytically activated and $220 \mathrm{mM}$ for thrombin-activated 
FXIIIA. Obtained at much lower centrifugal force than that used in the sedimentation velocity experiments, sedimentation equilibrium results provide critical support that activated FXIIIA is likely to exist in monomeric form at physiological concentrations.
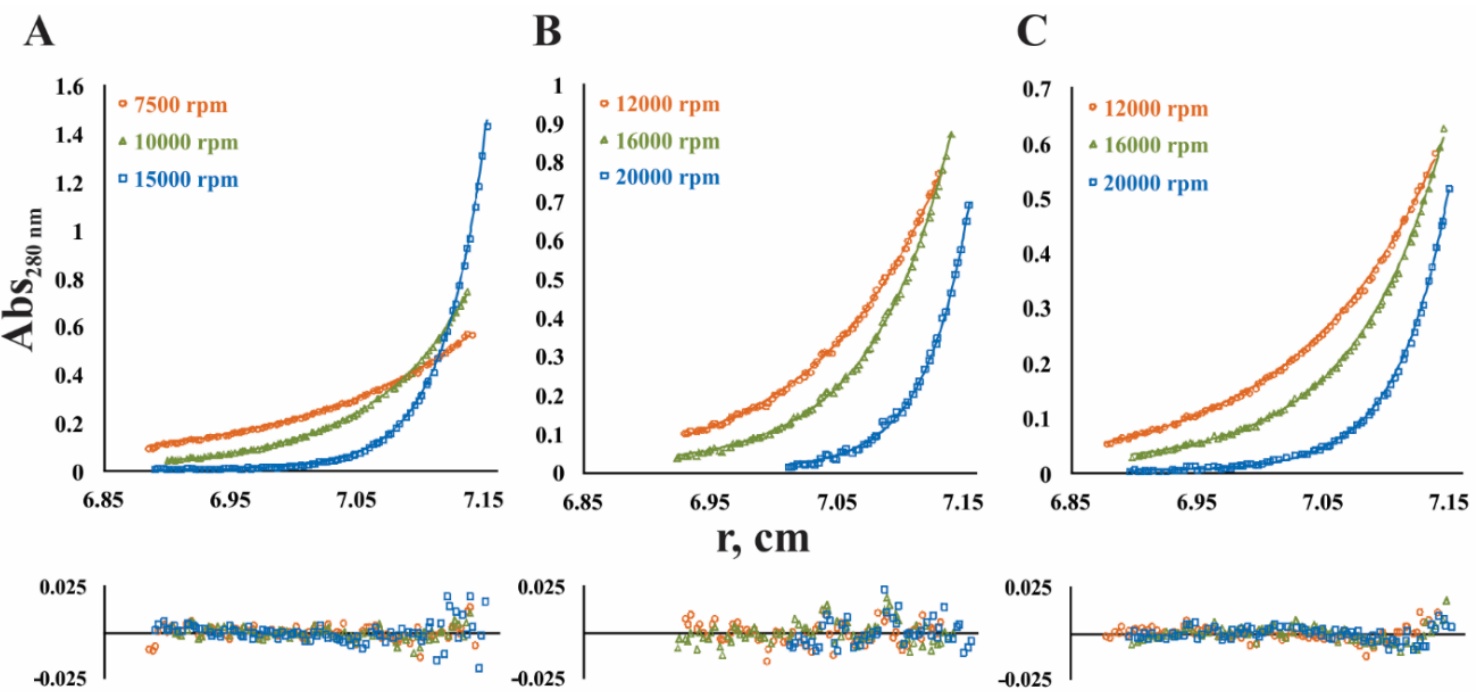

Fig. 22. Analysis of monomer-dimer equilibrium of FXIIIA forms. Sedimentation equilibrium profiles are presented for single loading concentration: (A) $2 \mu \mathrm{M}$ zymogen FXIIIA; (B) $4 \mu \mathrm{M}$ FXIIIA in the presence of $100 \mathrm{mM} \mathrm{CaCl}_{2}$; (C) $4 \mu \mathrm{M}$ FXIIIA activated by thrombin in the presence of $4 \mathrm{mM} \mathrm{CaCl}_{2}$. Experimental data (open symbols) obtained at each rotor speed (denoted on the figure panels) were fitted to a monomerdimer equilibrium model (solid lines). Residuals are provided below the fitted data. Apparent $K_{\mathrm{D}}$-values were $8 \mathrm{nM}$ for the zymogen FXIIIA, $90 \mathrm{mM}$ for FXIIIA in the presence of $100 \mathrm{mM} \mathrm{CaCl}_{2}$, and $220 \mathrm{mM}$ for the thrombin-activated FXIIIA. Global molecular weight values were 158,424 Da for zymogen, 86,801 for $\mathrm{CaCl}_{2}$-activated FXIIIA and 77,025 for thrombin-activated FXIIIA. Monte-Carlo error analysis indicated an average error for zymogen (A) of $\pm 30 \mathrm{Da}$ in molecular weight and \pm 0.03 for $\log K_{\mathrm{A}}$, for $\mathrm{CaCl}_{2}$-activated FXIII (B) of $\pm 40 \mathrm{Da}$ in molecular weight and \pm 0.003 for $\log K_{\mathrm{A}}$, and for thrombinactivated FXIII (C) of \pm 60 Da in molecular weight and \pm 0.002 for $\log K_{\mathrm{A}}$.

\section{Activated FXIIIA remains monomeric upon binding of substrate analogs}

To study possible structural rearrangements in the active FXIIIA upon binding substrate analogs, thrombin-activated enzyme was subjected to alkylation of the active site C314 by the thiol reagent IAA. As another test candidate, a peptide inhibitor K9-DON $\left({ }^{1} \mathrm{LGPG}-(\mathrm{DON})-\mathrm{SLVIG}^{10}\right)$, where glutamine is replaced with its isostere 6-diazo-5-oxonorleucine (85) was used to target the FXIIIA catalytic site region. These two covalent 
FXIIIA-inhibitor adducts resemble an acyl-enzyme intermediate transiently formed during the transglutaminase reaction. In the AUC experiments, both FXIIIA-IAA and FXIIIA-K9DON complexes appeared as monomers with no significant differences in the sedimentation coefficient compared to thrombin-activated free enzyme (Fig. 23).

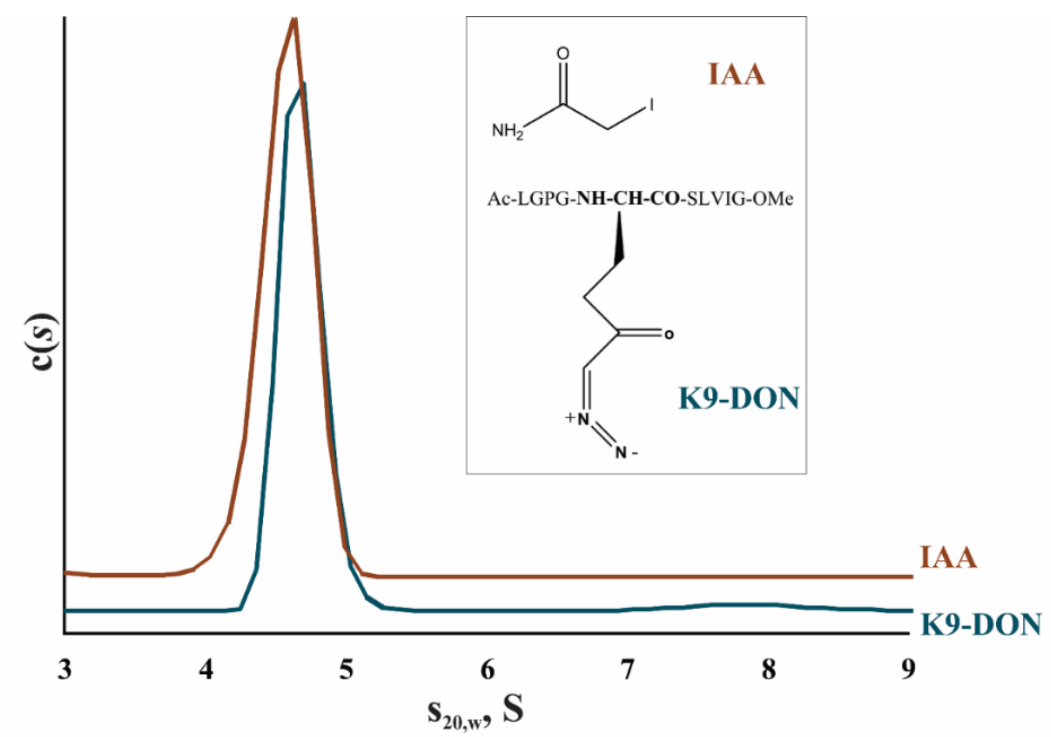

Fig. 23. Sedimentation velocity analysis of covalently inhibited FXIIIA. Structures of IAA and K9-DON are depicted in an insert.

\section{FXIIIA mutants with proposed impaired dimerization}

Certain single amino acid substitutions were previously predicted to impair dimerization of the zymogen FXIIIA form (74-79). To obtain solution evidence of such impairment in the current work, the expression of these mutants was attempted in E. coli. Following the autoinduction protocol described in Chapter 3, the GST-tagged FXIIIA mutants were affinity purified and liberated from the GST by on-column cleavage with PSP. The protein levels in eluted fractions were extremely low for R11Q, R260C, R260L, and G562R as judged by the absorbance at $280 \mathrm{~nm}$ (Fig. 24A, left), and undetectable by 
A
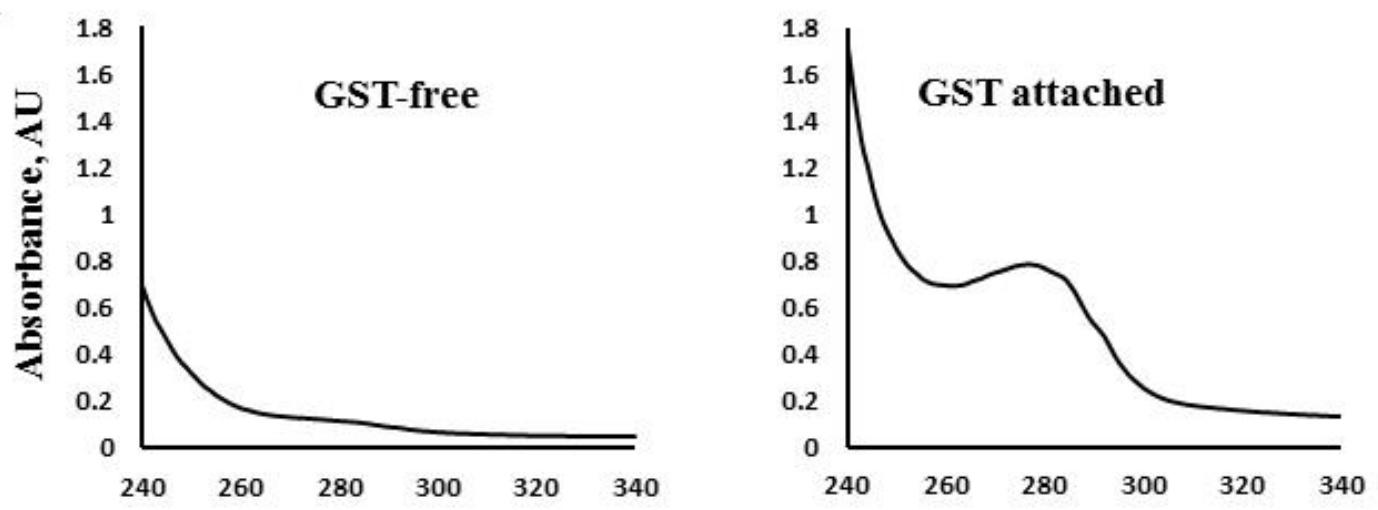

Wavelength, nm

B

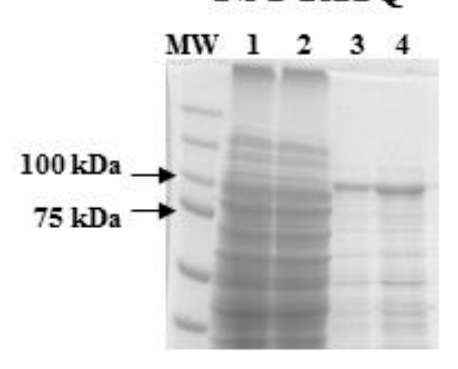

C

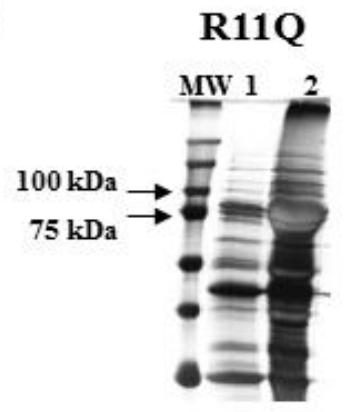

GST-R260C

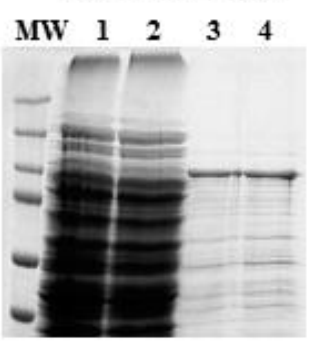

R260C

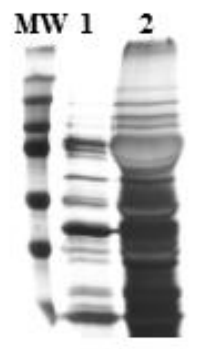

GST-G562R

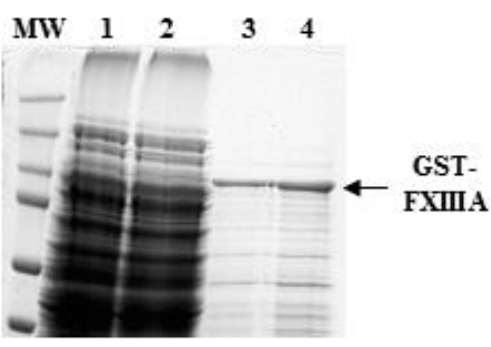

G562R

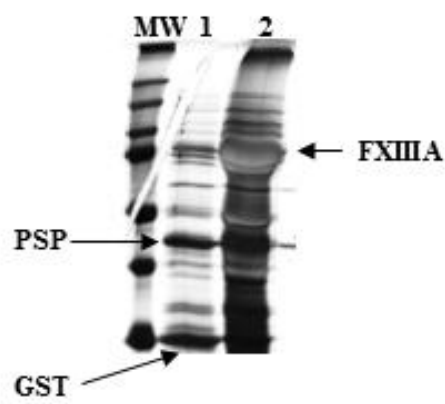

Fig. 24. Analysis of recombinantly expressed in E. coli FXIIIA mutants with proposed impaired dimerization of the zymogen. (A) Representative absorbance profiles of purified mutant proteins cleaved off the GST-tag (left) and purified as fusion proteins without cleavage of the tag. (B) SDS-PAGE analysis (8\% gels) of the GST-FXIIIA mutant proteins: MW - molecular weight standard, lane 1 - clarified bacterial lysate, lane 2 - column flow-through upon lysate load, lanes 3 and 4 - GST-FXIII containing fractions. (C) Purified GST-FXIIIA mutant proteins were subjected to PSP-mediated cleavage of the GSTtag in microfuge tubes and analyzed by $8 \%$ SDS-PAGE: MW - molecular weight standard, lane 1 - sample of FXIIIA mutant after cleavage the GST tag, lane 2 - sample of PSP-cleaved FXIIIA was centrifuged at $10,000 \mathrm{rpm}$, followed by resuspension of the pellet in sample loading buffer. 
the SDS-PAGE. These FXIIIA mutants were then purified without use of PSP and eluted off column with the covalently attached GST tag. As seen from Fig. 24A (right) and Fig. 24B, the GST-fused FXIIIA mutant proteins could be expressed in E. coli at reasonable levels. An increased (compared to the WT FXIIIA) amount of impurities detected by the SDS-PAGE was likely due to increased susceptibility of the mutants to intracellular proteolytic degradation, thus suggesting significant structural perturbations. Subsequent cleavage of the GST-tag (Fig. 24C) resulted in visible precipitation of R11Q, R260C, R260L, and G562R FXIIIA, thus further confirming solution instability of the mutants in the absence of the chaperoning GST tag.

Unlike the mutants described above, Y283C FXIIIA could be readily purified and eluted off the column without the GST tag, at levels comparable to the WT FXIIIA, with only minor impurities detected by the SDS-PAGE (Fig. 25 A, B). The nPAGE mobilities of Y283C FXIIIA and its hetero-complex with FXIIIB (Fig. 25C, lanes 4 and 5) were identical to those observed for the WT FXIIIA (Fig. 25C, lanes 1 and 3). The SEC elution profile of Y283C was, again, similar to the WT FXIIIA (Fig. 25D).

Additional information about the Y283C mutant was obtained in a series of AUC experiments (Fig. 25E). In the zymogen form, Y283C FXIIIA sedimented primarily as a dimer, although with a slightly lower sedimentation coefficient (7.6 S) than the WT FXIIIA (7.9 S). With WT and Y283C mutant having the same molecular weight, such a lower sedimentation coefficient indicated an increased asymmetry of the mutant FXIIIA molecule. A minor portion, $10 \%$ of total protein in the sample, had a sedimentation coefficient 4.9 S consistent with being a monomer. In the presence of $100 \mathrm{mM} \mathrm{Ca}^{2+}$, all protein was uniformly monomeric with the sedimentation coefficient of $4.9 \mathrm{~S}$, close to the 

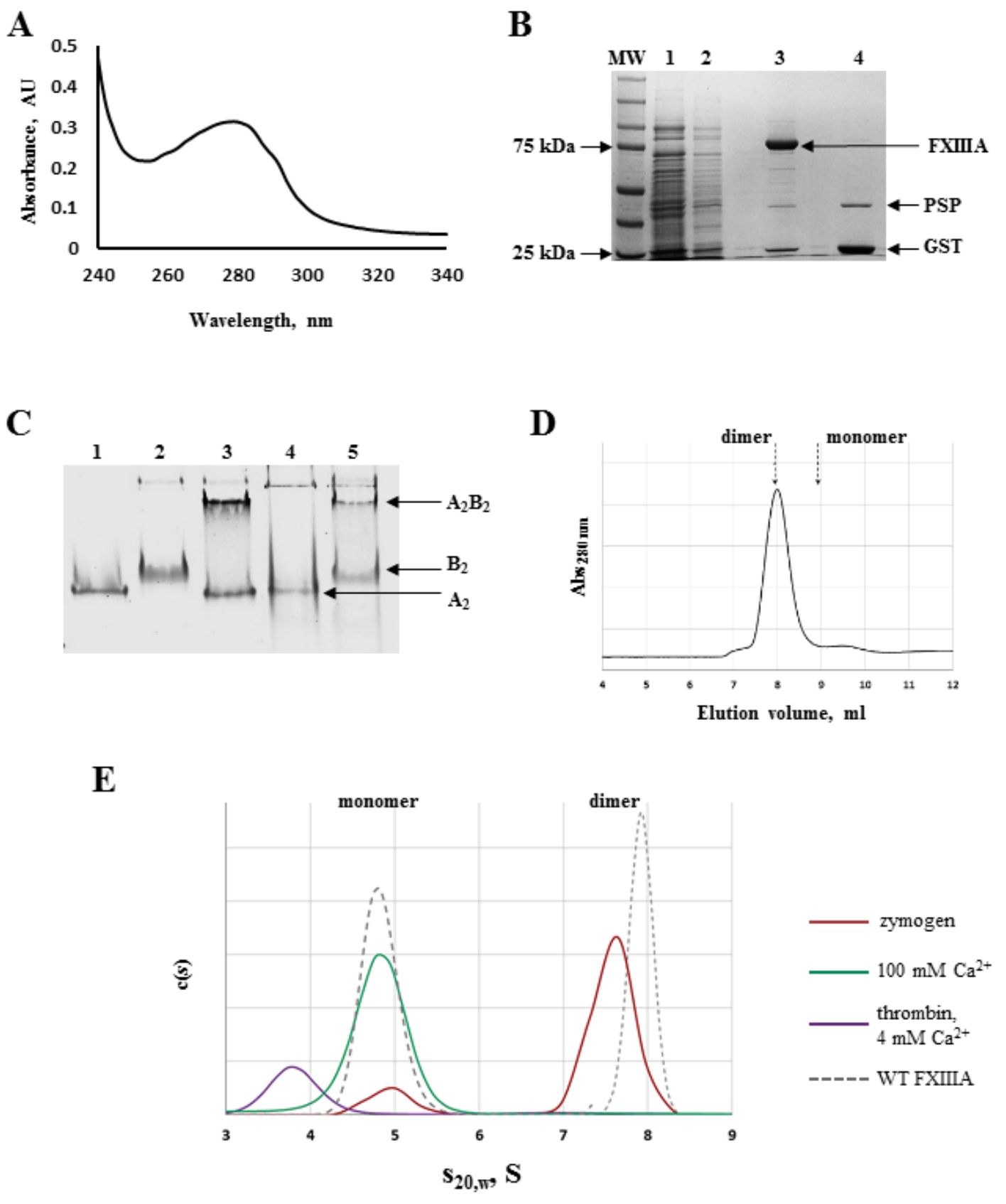

Fig. 25. Analysis of recombinantly expressed in E. coli FXIIIA mutant Y283C. (A) Representative absorbance profile of the mutant protein purified and cleaved off the GST-tag. (B) SDS-PAGE analysis ( $8 \%$ gel) of Y283C FXIIIA: MW - molecular weight standard, lane 1 - bacterial lysate, lane 2 - column flow-through upon lysate load, lane 3-Y283C protein, lane 4-GST-containing fraction. (C) nPAGE analysis of Y283C FXIIIA: lane 1 - control WT FXIIIA, lane 2 - control FXIIIB, lane 3 - WT FXIIIA (excess) incubated with FXIIIB, lane 4 - Y283C FXIIIA, lane 5-Y283C FXIIIA incubated with excess FXIIIB. (D) SEC elution profile of zymogen Y283C FXIIIA. Arrows indicate elution volumes of WT FXIIIA zymogen (dimer) and $100 \mathrm{mM} \mathrm{CaCl}_{2}$-treated FXIIIA (monomer) (E) Sedimentation velocity profiles of Y283C FXIIIA: zymogen form (red), Y283C in the presence of $100 \mathrm{mM} \mathrm{CaCL}_{2}$ (green), and thrombin cleaved Y283C in the presence of $4 \mathrm{mM} \mathrm{CaCl}_{2}$. For comparison, $\mathrm{c}(s)$ traces of WT FXIIIA zymogen (dimer) and in the presence of $100 \mathrm{mM} \mathrm{CaCl}_{2}$ (monomer) are plotted as dashed lines. 
WT FXIIIA $s$-value at these conditions (4.8 S). Interestingly, thrombin cleavage of AP on the Y283C FXIIIA resulted in a $3.9 \mathrm{~S}$ species. By analogy with the WT FXIIIA, such a large difference (1 S-unit) between sedimentation coefficients of the two Y283C monomeric forms would not be expected to result solely from proteolytic removal of a $4 \mathrm{kDa}$ activation peptide. Thus, the difference originated from the more global rearrangements present in the proteolytically cleaved Y283C FXIIIA than in the nonproteolytically dissociated mutant.

\section{Discussion}

Oligomeric assemblies of proteins, particularly those of enzymes, attracted the attention of investigators as soon as it was realized that such assemblies regulate protein function (125). With continuing advances in experimental techniques and instrumentation, the scope of protein oligomerization research has expanded significantly. It has been estimated (126) that $60 \%$ of enzymes form oligomers. Many of these enzymes may exist as monomers but require self-association to fulfill the catalytic function. By contrast, the spotlight of the current project is on a protein that is inactive in its oligomeric form.

In this work, the oligomerization states of the A-subunits of FXIII were evaluated under different solution conditions. Intrinsic tryptophan fluorescence profiles and electrophoretic mobilities of both proteolytically and non-proteolytically activated forms of FXIIIA were different from that of the control zymogen, suggesting significant perturbations to the protein upon activation. However, the attempt to resolve proteolytically and non-proteolytically activated FXIIIA using nPAGE encountered difficulties and did not yield defined protein bands on the gels. By contrast, similar experiments with 
homologous monomeric Transglutaminase 2 demonstrated two different forms. In nPAGE (127) as well as capillary electrophoresis (128), high mobility (compact conformation) and low mobility (extended conformation) species were observed. These species were correlated with the crystal structures of inactive and active enzyme, respectively.

A reasonable explanation for the current nPAGE results is that during separation in an electric field $\mathrm{Ca}^{2+}$ ions would be migrating in the opposite direction from the negatively charged protein, constantly "washing out" from the samples. Interestingly, the activation of FXIIIA by high concentrations of $\mathrm{Ca}^{2+}$ was previously shown to be a reversible process: loss of catalytic activity was observed upon removal of the $\mathrm{Ca}^{2+}$ ions from FXIIIA, and the protein could be re-activated again by addition of $\mathrm{Ca}^{2+}(129)$. Thus, nPAGE results in the current study indicate that presumably monomeric, activated FXIIIA might re-associate into zymogen-like dimers or higher-order aggregates upon loss of $\mathrm{Ca}^{2+}$ ions. This phenomenon occurred specifically in the presence of $\mathrm{Ca}^{2+}$ but not $\mathrm{Mg}^{2+}$, adding to the evidence that $\mathrm{Ca}^{2+}$ is critical for maintaining the conformation of active FXIIIA.

SEC analysis indicated different sizes (Stokes radii) of zymogen FXIIIA and its activated form. However, FXIIIA is not an ideal globular protein (Fig. 5, Chapter 1), therefore caution had to be taken when correlating SEC data with molecular weights of FXIIIA forms. By contrast, AUC provided information on the molecular weight and the shape of the protein in solution. The results of sedimentation velocity AUC experiments confirmed the dimeric state of zymogen FXIIIA 2 and its asymmetric shape, in accord with existing crystal structures and ultracentrifugation studies by Bishop et al. (105). FXIIIA activated proteolytically by thrombin in the presence of low $(4 \mathrm{mM}) \mathrm{Ca}^{2+}$ appeared as a monomer. Compared to the non-proteolytically $\mathrm{Ca}^{2+}$-activated form, the broader 
sedimentation coefficient peak distribution of thrombin-cleaved FXIIIA could indicate its more flexible conformation, and additional relevant experimentation is discussed in the following dissertation chapter.

Gupta and coworkers (87) conducted molecular dynamics simulations using a crystal structures of zymogen FXIIIA. Steered dissociation of the zymogenic dimer occurred within 60 ns. For simulation of proteolytic activation, the activation peptides were removed from the zymogen crystal structure, and the steered dissociation of A-subunits required only $30 \mathrm{~ns}$. Based on those time requirements, the Gupta group concluded that proteolytic FXIIIA activation causes dissociation of the A-subunits, while nonproteolytically activated FXIIIA was characterized as a "weak dimer". In the current work, solution based sedimentation equilibrium AUC provided a more apposite strategy to characterize inter-subunit interactions of FXIIIA forms quantitatively. The apparent dissociation constant for the zymogen FXIIIA was in the nanomolar range, whereas the value increased into the millimolar range for both proteolytically and nonproteolytically activated FXIIIA. To give an idea of the physiological range of FXIII concentrations in platelets, an established reference interval for FXIII $\mathrm{A}_{2}$ is $46-82 \mathrm{fg} / \mathrm{platelet}$ (103). With the mean platelet volume of $9 \mathrm{fL}(104)$, the concentration of the A-subunits is $61-110 \mu \mathrm{M}$. The plasma level of FXIII $\mathrm{A}_{2} \mathrm{~B}_{2}$ is $14-28 \mathrm{mg} / \mathrm{L}$ (102), corresponding to $86-172 \mathrm{nM}$ of the Asubunits. During a coagulation event, activated FXIII is mobilized at the clotting site and its local concentration may exceed regular plasma levels; however, the amount would not be expected to reach high millimolar values. Since dissociation of the B-subunits from the A-subunits after cleavage of the activation peptide has been well documented $(2,3)$, the physiological presence of FXIIIB is not predicted to significantly affect post-cleavage 
rearrangements of FXIIIA in plasma. Thus, the in solution observed drastic weakening of inter-subunit interactions in the current research supports the concept of physiological dissociation of FXIII A-subunits upon activation.

An observation of monomeric, thrombin-activated FXIIIA is contrary to previously reported results. Chung and coworkers (73) performed AUC of both plasma $\left(\mathrm{A}_{2} \mathrm{~B}_{2}\right)$ and platelet $\left(\mathrm{A}_{2}\right)$ FXIII using $50 \mathrm{mM}$ sodium citrate buffer. A valid concern from the Chung study is that most of the added $(10 \mathrm{mM}) \mathrm{Ca}^{2+}$ may have been chelated by citrate. Under their conditions, the dissociation of $\mathrm{B}_{2}$-dimer from the $\mathrm{A}_{2}$-dimer had occurred, but no evidence for the presence of monomeric FXIIIA either in plasma or platelet-derived enzyme was obtained, much like in the current nPAGE efforts. In the present study, no chelating agents were used in the buffers, thus $4 \mathrm{mM} \mathrm{Ca}^{2+}$ present in the samples was available for binding and stabilizing monomeric thrombin-cleaved FXIIIA (121).

In an X-ray crystal study, Yee et al. (122) reported no significant changes in structure of FXIIIA after activation with thrombin. However, non-cleaved FXIIIA zymogen was present in their crystallographic samples, which was described as a "minor component" (>20\%). Since thrombin-cleaved FXIIIA is prone to precipitation especially at the high concentrations $(10 \mathrm{mg} / \mathrm{ml})$ used, the Yee group may have actually crystallized the "minor component", zymogen FXIIIA, instead of the thrombin-cleaved form. Verification of the cleavage was not possible due to disordered nature of the cleavage site in FXIIIA activation peptide.

Earlier experiments employing radiolabeled IAA $(14,73,82)$ suggested that only half of the possible catalytic sites underwent alkylation, thus half-of-the-sites reactivity was postulated for the enzyme, implying its dimeric state. Upon observing active FXIIIA 
as a monomer in the present study, a possibility of re-association of the two active Asubunits upon binding an inhibitor to one of them was considered. To test this possibility, activated FXIIIA was modified with IAA or the more substrate-like peptide K9-DON to form covalent adducts with the active site C314 that mimic the acyl-enzyme complex. No dimerization was observed in sedimentation velocity analyses of both inhibited FXIIIA species. While there is no reason to dispute previous findings of the Folk (73) and Shafer $(14,82)$ research groups on half-of-the-sites reactivity of FXIIIA, there is an obvious need for a detailed study of possible cooperativity in FXIIIA-mediated catalysis.

In the dimeric FXIIIA zymogen, the outer, solvent exposed parts are formed mainly by the $\beta$-sheet structural elements that are less susceptible to proteolytic degradation, while the more vulnerable $\alpha$-helical structures are shielded within the dimer interface (Fig. 5, Chapter 1). At the same time, the active sites in the dimeric zymogen are buried, thus preventing premature activation and undesirable protein crosslinking. A few naturally occurring mutants causative of severe FXIIIA deficiency (R260C, R260L, Y283C, and G562R (75-78)) and an experimental activation peptide mutant R11Q (79) were predicted to be unable to dimerize and thus susceptible to intracellular proteolytic degradation. In the current work, one of those mutants, Y283C, could be successfully expressed and purified. The Y283 residue is located rather distant from the FXIIIA dimer interface (Fig. 7, Chapter 1), and its contribution to that interface would be modest. As observed in the SEC experiments, the Y283C substitution did not significantly affect the size of the FXIIIA molecule. AUC results indicated a local conformational distortion affecting overall shape of the A-subunits, but only slightly impaired dimerization of the zymogen. By contrast, R11, R260, and G562 residues directly participate in interactions of the A-subunits, and in 
the current work, mutations of those residues resulted in solution instability of FXIIIA. The mutants only remained soluble while still attached to the dimeric GST-tag. Collectively, these observations suggest that the dimeric state of FXIIIA is critical for stabilization of the zymogen form. In a physiological context, a high demand for FXIIIA in emergency of bleeding may not be quickly satisfied by inducible synthesis and extracellular secretion of such a large protein. Thus, a stand-by pool of FXIIIA molecules must be, and is, maintained in plasma in a dimeric zymogen form, that is protected from premature activation and degradation.

To summarize, current research provides experimental insight into FXIIIA oligomeric states in solution. For the first time, homodimeric FXIII $\mathrm{A}_{2}$ was demonstrated to dissociate as a part of the activation process, and inter-subunit interactions were quantitatively assessed. Current findings not only contribute to understanding the structure and function relationship in FXIIIA, but also provide a clue as to the regulation of this transglutaminase..$^{1}$

${ }^{1}$ Some contents of this chapter are reproduced from the published manuscript: Anokhin, B.A., Stribinskis, V., Dean, W.L., and Maurer, M.C. (2017) Activation of factor XIII is accompanied by a change in oligomerization state. FEBS J 284, 3849-3861. 


\section{CHAPTER 5}

\section{INVESTIGATION OF DYNAMIC AND FUNCTIONAL IMPLICATIONS OF FXIIIA ACTIVATION PATHWAYS}

\section{Introduction}

FXIIIA has been a subject of medically related research for almost a century. Perhaps the most studied is the role of FXIIIA in crosslinking the fibrin network. As physiological knowledge on FXIII expands, it becomes apparent that beyond serving as a key player of the blood coagulation system, FXIIIA functions in would healing, bone tissue dynamics, and even signaling processes $(2-5,21,48,49,54,130)$.

FXIIIA is a transglutaminase that crosslinks protein substrates via an isopeptide bond in a $\mathrm{Ca}^{2+}$-dependent manner. FXIIIA is expressed as an inactive zymogen and in plasma, it circulates in a heterotetrameric complex with carrier FXIIIB $\left(\mathrm{A}_{2} \mathrm{~B}_{2}\right)$. During blood coagulation, FXIII is activated by thrombin-mediated removal of $\mathrm{N}$-terminal activation peptides from the A-subunits followed by binding of $\mathrm{Ca}^{2+}$ and dissociation of the B-subunits (61). The active FXIIIA then introduces covalent crosslinks between polymerizing fibrin molecules and incorporates other proteins into the network, ultimately increasing mechanical stability of the resulting clot and its resistance to fibrinolysis (131). Intracellularly, such as in platelets, monocytes, and macrophages, FXIII exists as an $\mathrm{A}_{2}$-homodimer and is thought to undergo slow nonproteolytic activation in the presence

of low available $\mathrm{Ca}^{2+}$ concentrations (62-64). In this physiological compartment, FXIIIA 
is primarily present in the cytoplasm, sometimes associated with membrane (56), and even appears in the nucleus (9). Intracellular functions of FXIIIA include reorganization of cytoskeletal proteins and chromatin remodeling (5). FXIIIA is also secreted by osteoblasts into the ECM (57), where it contributes to formation of the ECM itself and remodeling of the bone tissue $(49,55,56)$. Although a proteolytic fragment of FXIIIA that results from posttranslational intracellular modification has been detected in the ECM $(47,57)$, it was later identified as transaldolase 1 (53). With available $\mathrm{ECM} \mathrm{Ca}^{2+}$ concentrations as high as $25-40 \mathrm{mM}(45,132)$, bone ECM FXIIIA may be activated by $\mathrm{Ca}^{2+}$ without proteolysis.

Being involved in an array of intra- and extracellular events, FXIIIA has also been implicated in associated pathological conditions such as thrombotic disease (17), arthritis (41), etc. FXIIIA has thus become an attractive target for pharmacological intervention, and development of therapeutic regimes effectively managing FXIIIA in its particular physiological environments warrants an in-depth understanding of FXIIIA activation and function in such locations. Solution studies discussed in the previous chapter revealed that FXIII $\mathrm{A}_{2}$-homodimer dissociates into monomers as part of the activation process. Research focused next on probing the functional implications of FXIIIA activation pathways: (i) thrombin activation of FXIIIA in the presence of low $\mathrm{mM} \mathrm{Ca}^{2+}$ (occurring in plasma, the resulting active species are henceforth denoted as FXIIIA*), (ii) nonproteolytic activation by low $\mathrm{mM} \mathrm{Ca}^{2+}$ (intracellular activation, FXIIIA ${ }^{\circ \text { low }}$ ), and (iii) activation in the presence of high ( $\geq 25 \mathrm{mM}) \mathrm{Ca}^{2+}$ (bone ECM, FXIIIA ${ }^{\circ \text {,high }}$ ). 


\section{Materials and Methods}

Materials

Recombinant FXIIIA expressed in S. cerevisiae was a gift from the late Dr. Paul Bishop (Zymogenetics, Seattle, WA). Bovine thrombin was purchased from SigmaAldrich. Recombinant human thrombin was generously provided by Dr. Enrico Di Cera and Dr. Leslie Pelc (St. Louis University) Thrombin inhibitor PPACK was from Haematologic Technologies (Essex Junction, VT). A glutamine-containing substrate peptide K9 was synthesized by New England Peptide (Gardner, MA), stock solutions (15-30 mM) were made in deionized $\mathrm{H}_{2} \mathrm{O}$, and accurate concentration was determined by quantitative amino acid analysis (AAA Service Laboratory, Damascus, OR). Fibrinogen $\alpha \mathrm{C}$ (233-425) was recombinantly expressed in E. coli using autoinduction media and purified as described in Chapter 3. Bovine liver glutamate dehydrogenase and NADH were from Roche. All other reagents were from Sigma-Aldrich.

\section{FXIIIA activation for AUC experiments}

For full proteolytic activation, $2 \mu \mathrm{M}$ FXIIIA 2 was incubated in borate buffer (20 mM boric acid, $\mathrm{pH} 7.8,150 \mathrm{mM} \mathrm{NaCl})$ with bovine thrombin $(3.5 \mathrm{NIH}$ units/ml) and $4 \mathrm{mM} \mathrm{CaCl}_{2}$ for $30 \mathrm{~min}$ at $37{ }^{\circ} \mathrm{C}$. To achieve a limited amount of proteolysis (cleavage of approximately half of the A-subunits), the zymogen $(2 \mu \mathrm{M})$ was incubated with $3.5 \mathrm{NIH}$ $\mathrm{U} / \mathrm{mL}$ thrombin for $6 \mathrm{~min}$ at $28^{\circ} \mathrm{C}$. All proteolytic activation reactions were quenched with $760 \mathrm{nM}$ PPACK. In addition to $4 \mathrm{mM} \mathrm{CaCl}_{2}$, some samples of the proteolytically activated FXIIIA were supplemented with $100 \mathrm{mM} \mathrm{MgCl}_{2}$.

For nonproteolytic activation, $2 \mu \mathrm{M}$ zymogen FXIIIA $_{2}$ was incubated in the borate 
buffer containing $4-100 \mathrm{mM} \mathrm{CaCl}_{2}$ for $30 \mathrm{~min}-6 \mathrm{~h}$ at $37^{\circ} \mathrm{C}$. FXIIIA was also incubated in the borate buffer at a lower, $4 \mathrm{mM} \mathrm{CaCl}_{2}$ for $30 \mathrm{~min}-96 \mathrm{~h}$. To achieve better solubility of FXIIIA at these conditions, the $4 \mathrm{mM} \mathrm{Ca}^{2+}$-samples were supplemented with 5\% DMSO. A detailed description of AUC experimental parameters can be found in Chapter 4. Briefly, samples of activated FXIIIA were subjected to sedimentation velocity AUC at $20^{\circ} \mathrm{C}$ and 50,000 rpm. Data were analyzed using the program Sedfit and experimental sedimentation coefficients were corrected based on the measured density and viscosity of the buffer, thus allowing direct comparison of results obtained with different experimental conditions. Analytical runs were performed with at least two independent samples for each condition examined.

Coupled ammonia release activity assay

Transglutaminase activity of FXIIIA was determined using a standard coupled spectrophotometric assay as described in Chapter 3. The only alteration was maintaining the $\mathrm{CaCl}_{2}$ concentration at $4 \mathrm{mM}$ for the thrombin-activated FXIIIA and $100 \mathrm{mM}$ for the non-proteolytically activated enzyme. Activity measurements were performed in triplicates.

Monodansylcadaverine (MDC) crosslinking activity assay

In order to compare activities of proteolytically and nonproteolytically activated FXIIIA, a series of MDC crosslinking assays was performed. For proteolytic activation, $1 \mu \mathrm{M}$ FXIIIA was incubated in TBS (50 mM Tris Acetate, $\mathrm{pH} 7.4,150 \mathrm{mM} \mathrm{NaCl}$ ) with $30 \mathrm{nM}$ recombinant human thrombin and $4 \mathrm{mM} \mathrm{CaCl}_{2}$ for $30 \mathrm{~min}$ at $37^{\circ} \mathrm{C}$, followed by 
addition of $760 \mathrm{nM}$ thrombin inhibitor PPACK. Nonproteolytic activation was achieved by 30 min incubation of $1 \mu \mathrm{M}$ FXIIIA in TBS in the presence of $100 \mathrm{mM} \mathrm{CaCl}_{2}$.

Recombinantly expressed fibrinogen $\alpha \mathrm{C}(233-425)$ was used as a glutamine donor (Q-substrate). $\alpha \mathrm{C}(2-40 \mu \mathrm{M}$ final) was preincubated with a lysine mimic MDC (Ksubstrate, $1 \mathrm{mM}$ final, from a $20 \mathrm{mM}$ stock solution in methanol) in TBS containing $\mathrm{CaCl}_{2}$ (4-100 $\mathrm{mM}$ final concentration) for $5 \mathrm{~min}$ at $37^{\circ} \mathrm{C}$. The crosslinking reaction was initiated by addition of activated FXIIIA (final concentration $100 \mathrm{nM}$ ). Aliquots were taken at 1 $7 \mathrm{~min}$ and quenched by addition of reducing sample loading buffer, followed by $3 \mathrm{~min}$ of boiling. Samples were resolved using SDS-PAGE on $15 \%$ gels. Prior to Coomassie blue staining, the gels were photographed under UV light. This assay was performed at least twice for each condition studied.

\section{Direct continuous chromogenic activity assay}

In this assay adapted from de Macedo et al. (133), FXIIIA incorporated chromogenic K-substrate $N, N$-dimethyl-1,4-phenylenediamine (DMPDA) into a Qsubstrate K9-peptide $\left({ }^{1} \mathrm{LGPGQSKVIG}^{10}\right)$. The reaction progress was monitored by an increase in absorbance at $278 \mathrm{~nm}$ resulting from an anilide functionality in the crosslinked product. Nonproteolytic and proteolytic activation was performed as described for the MDC assay, except that FXIIIA was 1-4 $\mu \mathrm{M}$ in the activation mix. K9-peptide (46$1386 \mu \mathrm{M}$ final) was preincubated with DMPDA (700 $\mu \mathrm{M}$ final, from a $100 \mathrm{mM}$ stock solution in methanol) in the borate buffer with 4 or $100 \mathrm{mM}$ of $\mathrm{CaCl}_{2}$ for $5 \mathrm{~min}$ at $37{ }^{\circ} \mathrm{C}$. The reaction was initiated by addition of activated FXIIIA (final concentration $0.25-1.0 \mu \mathrm{M})$. Reaction velocities were determined over the initial part of the absorbance 
curve. An $\varepsilon=8940 \mathrm{M}^{-1} \mathrm{~cm}^{-1}$ (133) was applied to convert absorbance to $\mu \mathrm{M}$ of crosslinked product. Velocities in $\mu \mathrm{M}$ product/min were fitted to the Michaelis-Menten equation as a function of the K9 concentrations using Kaleidagraph software (Synergy).

\section{Results}

\section{Dynamics of proteolytic activation of FXIIIA}

In the previous chapter, FXIII $\mathrm{A}_{2}$ was demonstrated to dissociate into monomers upon activation by thrombin. Under the conditions employed, FXIIIA was fully cleaved by thrombin within 30 min. Previously published kinetic studies by Hornyak et al. (14) suggested that only one activation peptide has to be cleaved on the FXIIIA 2 dimer to expose the active sites in both subunits in the presence of $\mathrm{Ca}^{2+}$. To assess the oligomerization state of this "single-cleaved" FXIIIA*, activation conditions were adjusted so that approximately half of the A-subunits were cleaved by thrombin (Fig. 26A). A series of samples was prepared, and some of them were supplemented with 5\% DMSO to promote solubility of the monomeric FXIIIA* species. Representative AUC results for these "halfcleaved" samples are depicted on Fig. 26C. From a quantitative analysis of sedimentation velocity data of one of the samples (without DMSO), $48 \%$ of the protein appeared as a monomer, $42 \%$ was a dimer, and $10 \%$ formed a large aggregate. Another sample was supplemented with 5\% DMSO, and no aggregation was observed yielding 62\% monomer and $38 \%$ dimer. Assuming random cleavage by thrombin, the resulting distribution of species in these samples (Fig. 26B) would be $\sim 25 \% \mathrm{~A}_{2}$ (non-cleaved zymogen) : 50\% $\mathrm{A}^{\prime} \mathrm{A}$ ("single-cleaved") : 25\% A' (“double-cleaved"). Hence, the "double-cleaved" species would constitute $\sim 25 \%$ of monomers in the samples. Current observation of $48 \%$ (with no 
DMSO) and 62\% (in the presence of 5\% DMSO) monomeric protein can be accounted for by assuming dissociation of a major portion of the "single-cleaved" FXIIIA dimers.

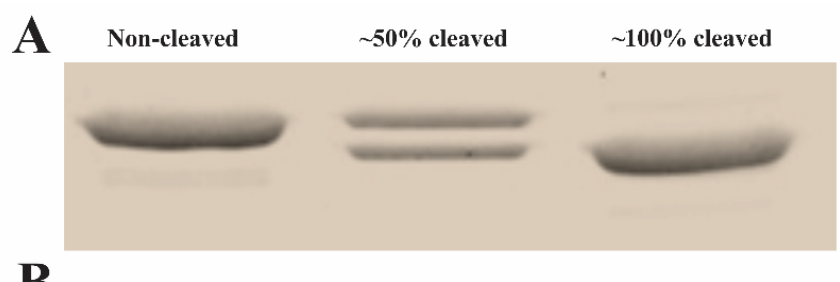

B
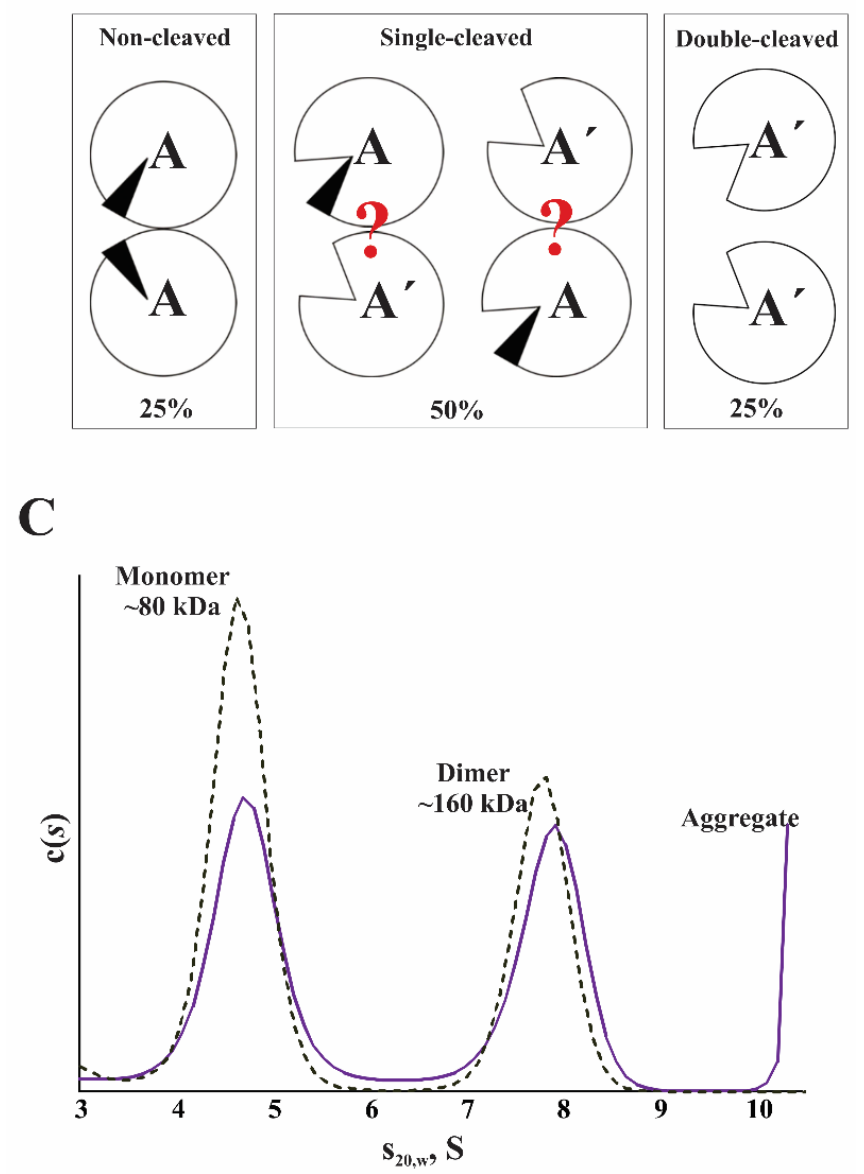

Fig. 26. Analysis of monomer-dimer distribution of FXIIIA partially activated by thrombin in the presence of $4 \mathrm{mM} \mathrm{CaCl}$. (A) SDS-PAGE (8\% gel) monitoring cleavage of FXIIIA by thrombin. (B) Theoretic distribution of non-cleaved and cleaved species in the "half-cleaved" samples of FXIIIA. Question marks denote unknown oligomerization state of the "single-cleaved" species. (C) Representative c $(s)$ distribution in the "half-cleaved" samples with (dashed line) and without (solid line) addition of 5\% DMSO. 


\section{Dynamics of nonproteolytic activation of FXIIIA}

Similar to the proteolytic activation by thrombin, FXIIIA activation in the presence of $100 \mathrm{mM} \mathrm{Ca}^{2+}$ led to a complete dissociation of the A-subunits within 30 min (Chapter 4). For a more detailed assessment of FXIIIA non-proteolytic activation dynamics, the protein was treated for $30 \mathrm{~min}$ in the presence of different concentrations of $\mathrm{Ca}^{2+}$. The samples were then subjected to AUC. The resultant sedimentation velocity data were used to quantitate the amount of FXIIIA monomers depending on the concentration of $\mathrm{Ca}^{2+}$ (Fig. 27). As expected, increasing $\mathrm{Ca}^{2+}$ concentrations resulted in a greater amount of monomers.

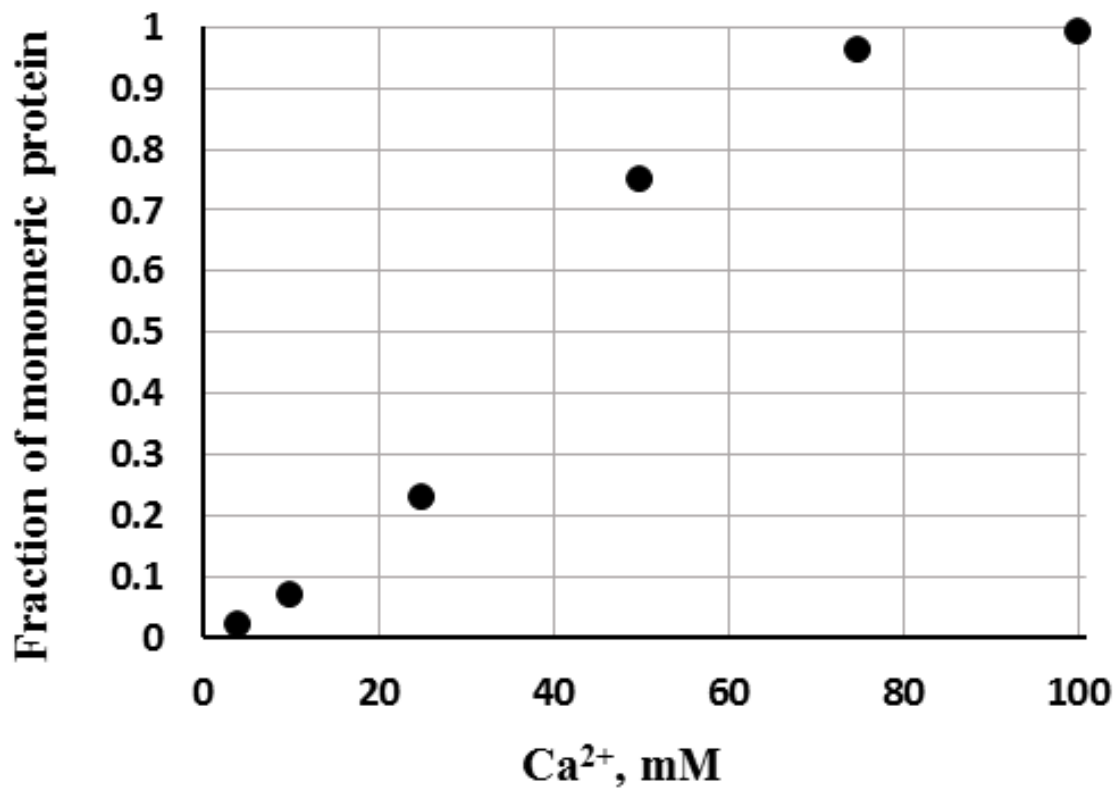

Fig. 27. FXIIIA dissociation as a function of $\mathrm{Ca}^{2+}$ concentration. All samples were incubated at $37^{\circ} \mathrm{C}$ for $30 \mathrm{~min}$.

There was now an interest in examining the FXIIIA nonproteolytic activation process at lower, more physiological $\mathrm{Ca}^{2+}$ concentrations. FXIIIA was incubated in the presence of $4 \mathrm{mM} \mathrm{Ca}^{2+}$ for $30 \mathrm{~min}-96 \mathrm{~h}$, and all samples were subjected to AUC in the same run. The amount of protein in solution was assessed at the start of an AUC 
experiment. Unexpectedly, progressive precipitation was observed in these samples of FXIIIA $^{\circ \text {,low }}$, very similar to the solution behavior of the thrombin-activated FXIIIA*. In previous experiments, addition of 5\% DMSO promoted solubility of FXIIIA* over the course of a few hours. However, low $\mathrm{mM} \mathrm{Ca}{ }^{2+}$ activation required longer incubation periods to obtain detectable results, and even addition of DMSO did not aid in full solubility of FXIIIA ${ }^{\circ \text {,low }}$. As seen in Fig. 28A, approximately $60 \%$ of the protein was insoluble by $96 \mathrm{~h}$ of incubation with $4 \mathrm{mM} \mathrm{Ca}^{2+}$. By contrast, in a control sample of zymogen FXIIIA (in the absence of $\mathrm{Ca}^{2+}$ ), only $9 \%$ of the protein was lost to aggregation at that incubation time (data not shown).

The plot in Fig. 28C (open circles) represents the amount of monomeric FXIIIA as a fraction of soluble protein in each sample. The most observed dissociation of the Asubunits, $\sim 65 \%$, was observed after $72 \mathrm{~h}$ incubation. This amount is likely underestimated, since in the presence of low $\mathrm{Ca}^{2+}$ concentrations it is the monomeric FXIIIA that is prone to precipitation, as seen in experiments with FXIIIA*. Such activation of FXIIIA may proceed intracellularly, although much slower than observed in the presence of $4 \mathrm{mM} \mathrm{Ca}^{2+}$, since $\mathrm{Ca}^{2+}$ is actually maintained at $20-200 \mathrm{nM}$ level in most resting cells (134) and can be elevated to $\sim 1 \mathrm{mM}$ in activated platelets $(62,63)$.

In order to mimic activation conditions of bone tissue ECM, FXIIIA was incubated in the presence of higher, $25 \mathrm{mM} \mathrm{Ca}^{2+}$ for $30 \mathrm{~min}-6 \mathrm{~h}$ (Fig. $28 \mathrm{~B}$ and C, filled circles). The dissociation of the dimeric zymogen under these conditions occurred significantly faster than at $4 \mathrm{mM} \mathrm{Ca}^{2+}(80 \%$ at $6 \mathrm{~h}$ of incubation) and, analogous to activation by 100 $\mathrm{mM} \mathrm{Ca}^{2+}$, no precipitation of these $\mathrm{FXIII}^{\circ}{ }^{\text {,high }}$ species was observed. Thus, current results 

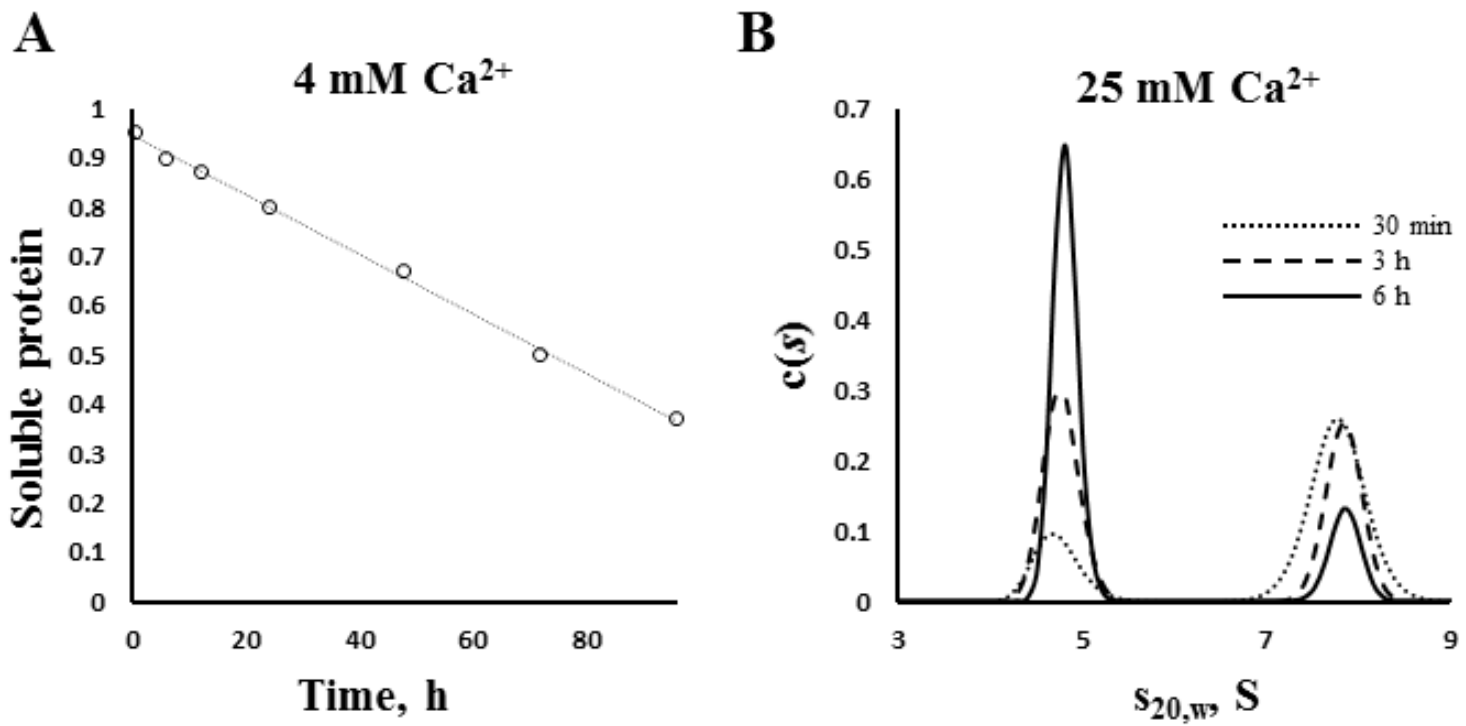

C

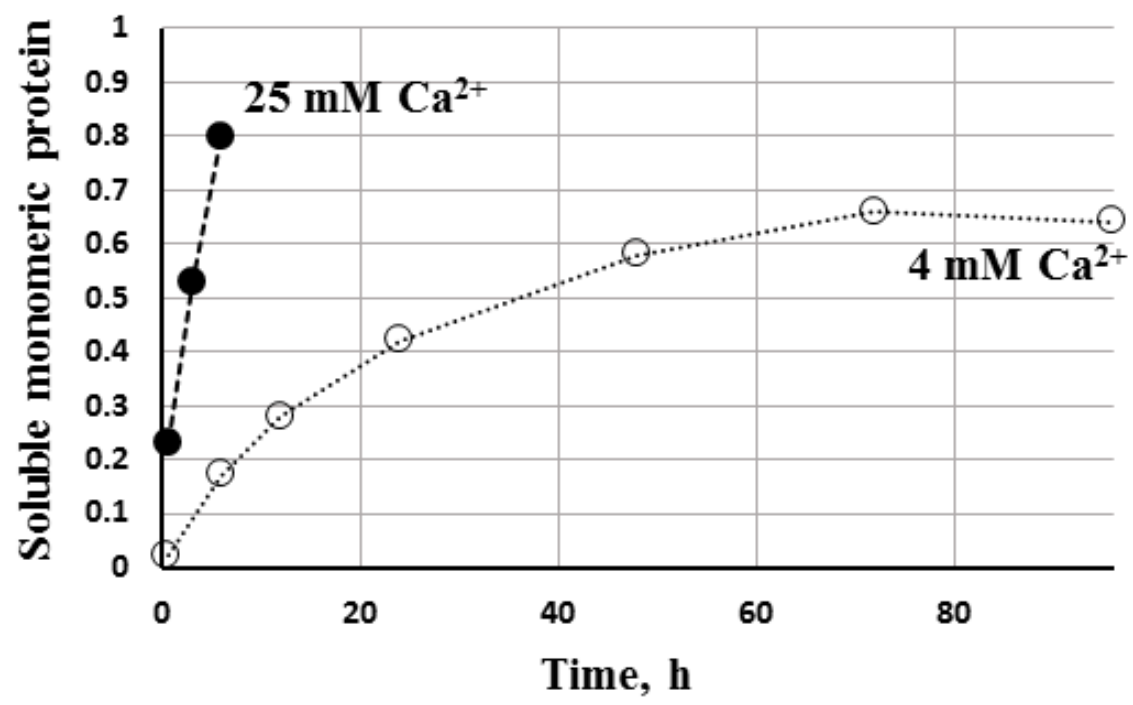

Fig. 28. Dynamics of FXIIIA dissociation in the presence of 4 (FXIIIA ${ }^{\circ \text {,low }}$, open circles) and 25 (FXIIIA ${ }^{\circ \text {,high }}$, filled circles) $\mathrm{mM} \mathrm{CaCl}_{2}$. (A) Assessment of soluble protein amount (fraction of total) in the samples of FXIIIA ${ }^{\circ \text {,low }}$ (B) Representative c(s) distributions of FXIIIA ${ }^{\circ \text {,high }}$ activated in the presence of $25 \mathrm{mM} \mathrm{CaCl}{ }_{2}$ for $30 \mathrm{~min}$ (dotted line), $3 \mathrm{~h}$ (dashed line), and $6 \mathrm{~h}$ (solid line). (C) Amount (fraction of soluble) of monomeric protein in the samples of FXIIIA ${ }^{\circ \text {,low }}$ (open circles) and FXIIIA ${ }^{\circ \text {,high }}$ (filled circles).

indicate that nonproteolytic activation of FXIIIA may readily be achieved at much lower $\mathrm{Ca}^{2+}$ concentrations than previously thought $(50 \mathrm{mM})$. In physiological conditions, such a mechanism is certainly expected to proceed slower than proteolytic activation by thrombin, 
given that cleavage of a single activation peptide on the dimeric zymogen is often sufficient to activate both A-subunits.

\section{Solution properties of FXIIIA species in different activation conditions}

AUC results discussed in the previous chapter suggested differences in the conformational flexibility of FXIIIA* (activated by thrombin in the presence of $4 \mathrm{mM}$ $\mathrm{Ca}^{2+}$ ) and FXIIIA ${ }^{\circ, \text { high }}$ (activated in the presence of $100 \mathrm{mM} \mathrm{Ca}^{2+}$ ). There was now an interest in directly comparing the solution behavior of these two active FXIIIA forms. In the current investigation, a necessary control for a divalent cation-mediated ionic strength effect was provided by supplementing FXIIIA* samples with $100 \mathrm{mM} \mathrm{Mg}^{2+}$. Unlike $\mathrm{Ca}^{2+}$, this divalent cation does not efficiently support dissociation or activity of FXIII A-subunits. In addition, to eliminate possible differences in sample handling between separate analytical runs, all sample conditions were subjected to AUC at the same time.

The resulting sedimentation coefficient distributions are presented in Fig. 29. In the presence of $100 \mathrm{mM} \mathrm{Ca}^{2+}$ (trace i), we observed a tall narrow peak, approximately $1 \mathrm{~S}$ unit in width. Significant precipitation occurred in FXIIIA* samples during centrifugation, and the sedimentation distribution peak was much broader, approximately $2 \mathrm{~S}$ (trace ii). In the presence of $4 \mathrm{mM} \mathrm{Ca}^{2+}$ and $100 \mathrm{mM} \mathrm{Mg}^{2+}$ (trace iii), the FXIIIA* sedimentation peak was also broad (2 S). Sedimentation coefficients represent the velocity of particle sedimentation (in the present case, FXIIIA molecules) in a centrifugal field. Apart from the physical characteristics of a solution, which are accounted for in data analysis, the sedimentation coefficient ultimately depends on the weight and shape of those particles. Thus, in samples of monomeric FXIIIA, it is the diversity of shapes (conformations) that constitutes the 
sedimentation distribution. The broad sedimentation distribution peak indicates that FXIIIA* is more conformationally heterogeneous in solution than FXIIIA ${ }^{\circ \text {,high }}$, and this heterogeneity is retained in the presence of $100 \mathrm{mM} \mathrm{Mg}^{2+}$. This observation suggests a specific effect of $\mathrm{Ca}^{2+}$ on FXIIIA conformation rather than a simple increase in ionic strength due to the higher concentration of divalent cation.

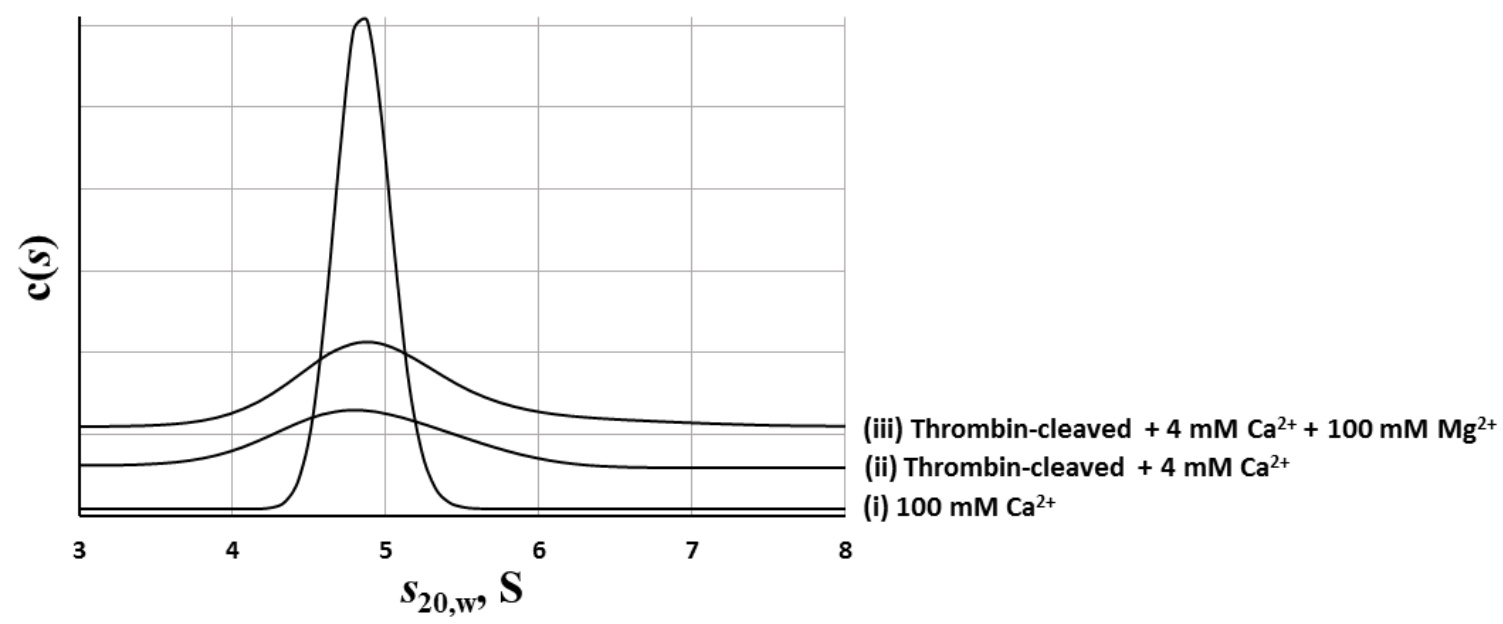

Fig. 29. Comparison of $\mathrm{c}(s)$-distributions for activated FXIIIA: (i) FXIIIA ${ }^{\circ \text {,high }}$, (ii) FXIIIA*, (iii) same as (ii) with addition of $100 \mathrm{mM} \mathrm{MgCl}$. For each condition, two independent samples were prepared. All six samples were analyzed in the same centrifugation run.

Catalytic activities of proteolytically and non-proteolytically activated FXIIIA

The transglutaminase activity of FXIIIA under different activation conditions was first compared using a standard coupled spectrophotometric assay $(85,114,115)$. As a brief description of the assay, FXIIIA incorporates a primary amine glycine ethyl ester (GEE) into a glutamine-containing K9-peptide with the release of ammonia. Glutamate dehydrogenase utilizes this ammonia along with NADH to convert $\alpha$-ketoglutarate to glutamate, accompanied by a sigmoidal decrease in absorbance at $340 \mathrm{~nm}$ (Fig. 30A). The reaction velocity is determined as a slope at the steepest part of the absorbance curve. 
A
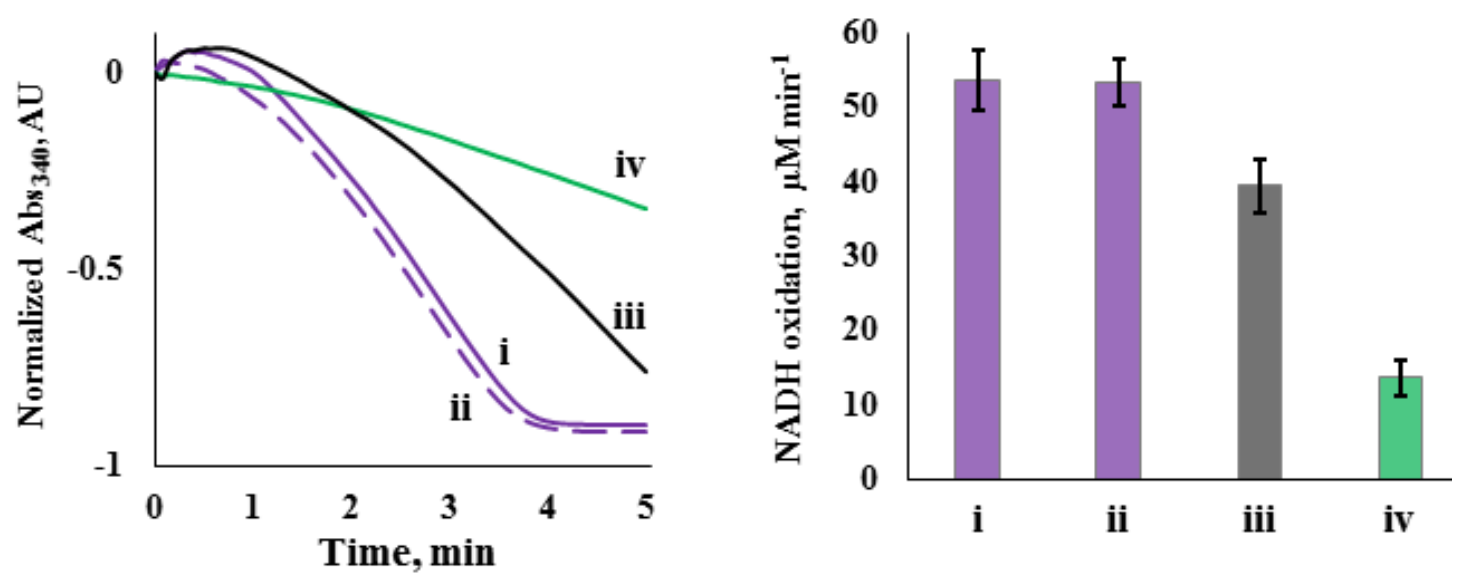

B

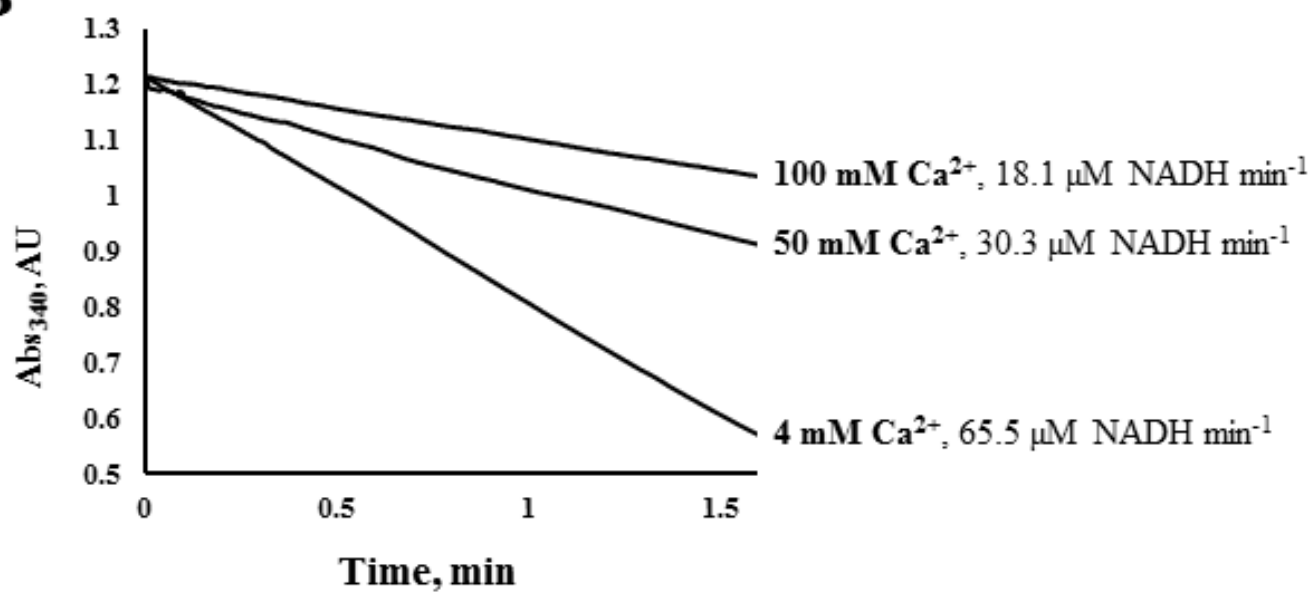

Fig. 30. Coupled ammonia release assay of FXIIIA transglutaminase activity. (A) Representative absorbance curves monitoring FXIIIA-mediated ammonia release followed by oxidation of NADH (left): (i) - FXIIIA* fully cleaved by thrombin, (ii) - same as (i), with addition of 5\% DMSO, (iii) - FXIIIA* sample with $\sim 50 \%$ of the A-subunits cleaved by thrombin, (iv) - FXIIIA ${ }^{\text {o,high }}\left(100 \mathrm{mM} \mathrm{CaCl}_{2}\right)$. Transglutaminase activity of FXIIIA is presented by the rate of ammonia-dependent NADH oxidation (right). Measurements were performed in triplicates for each condition, and values are presented as mean \pm SD. (B) Control experiments (with no FXIIIA) monitoring NADH oxidation by GDH in the presence of different $\mathrm{CaCl}_{2}$ concentrations. Reactions were initiated by addition of $\mathrm{NH}_{4} \mathrm{Cl}$.

In previous experiments, 5\% DMSO facilitated solubility of thrombin-cleaved FXIIIA*, however this co-solvent could affect conformation and hence, catalytic activity of the enzyme. As seen from Fig. 30A (ii), addition of 5\% DMSO did not affect transglutaminase activity of the FXIIIA*. The activity of samples with half of the A- 
subunits cleaved by thrombin (Fig. 30A, iii) was $\sim 3 / 4$ of that observed with the fullycleaved FXIIIA*, consistent with previous reports $(14,105)$. Surprisingly, enzyme fully dissociated in the presence of $100 \mathrm{mM} \mathrm{Ca}^{2+}\left(\mathrm{FXIIIA}^{\text {o,high }}\right)$ yielded much lower activity (Fig. 30A, iv) than fully activated FXIIIA*. Additional control experiments, however, revealed that high $\mathrm{mM} \mathrm{Ca}{ }^{2+}$ had an inhibitory effect on the activity of the coupling enzyme glutamate dehydrogenase (GDH). As seen from Fig. 30B, the GDH activity in the presence of $100 \mathrm{mM}$ is $\sim 3.5$ fold less than at $4 \mathrm{mM} \mathrm{Ca}^{2+}$, a difference identical to that observed with FXIIIA* $\left(4 \mathrm{mM} \mathrm{Ca}^{2+}\right)$ and FXIII ${ }^{\text {,high }}\left(100 \mathrm{mM} \mathrm{Ca}^{2+}\right)$.

For a more reliable assessment of the possible catalytic differences between FXIIIA* and FXIIIA $^{\circ \text {,high }}$, a direct spectrophotometric transglutaminase assay, developed by de Macedo with coworkers (133) was adapted. With the K9 peptide still serving as a glutamine substrate, the chromogenic DMPDA was used as the second, amine substrate. Unlike the coupled assay based on detection of released ammonia, the transglutaminase reaction in this method is monitored by appearance of crosslinked product that absorbs at $278 \mathrm{~nm}$ (Fig. 31A). More important, this assay is not sensitive to high $\mathrm{Ca}^{2+}$ concentrations.

At first glance, the downward curvature of the Lineweaver-Burk plots, such as the one in Fig. 31B (left), suggested that initial reaction velocities could not saturate, in other words, they did not approach the $V_{\text {max }}$. Such a response of activated FXIIIA forms to increasing K9-peptide concentrations would be characteristic of negative cooperativity. Noteworthy, half-of-the-sites reactivity, an extreme case of negative cooperativity, was previously reported for irreversible covalent inhibition of FXIIIA $(14,73,82)$. However, since each FXIII A-subunit contains one active site, half-of-the-sites activity 
A

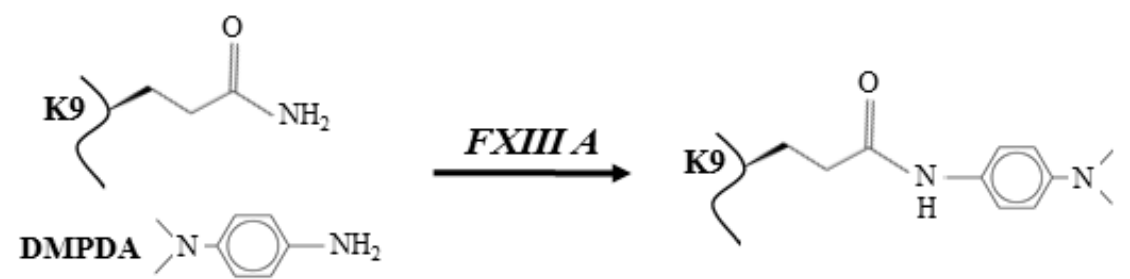

B
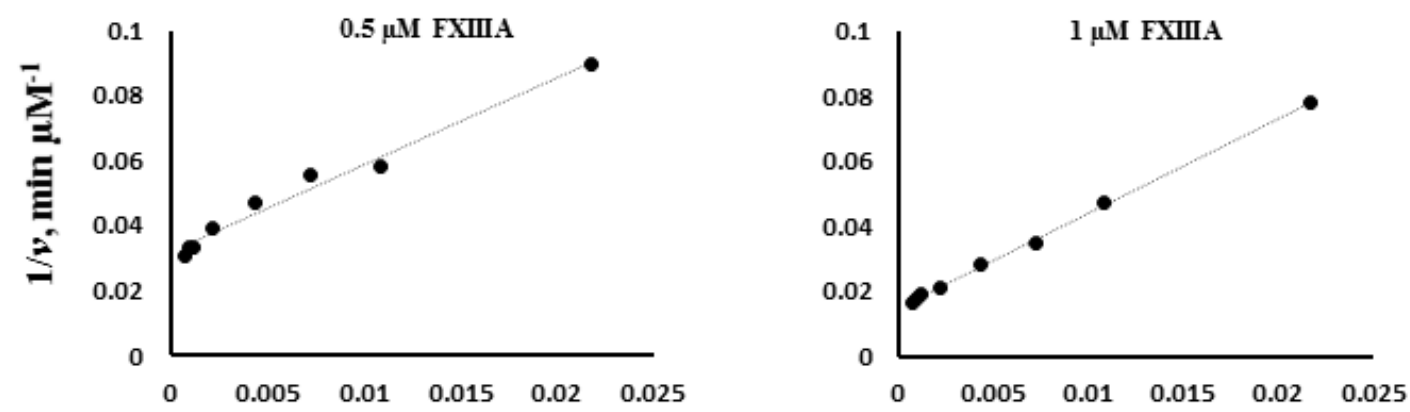

$\mathbf{1} / \mathrm{S}], \mu \mathbf{M}^{-1}$

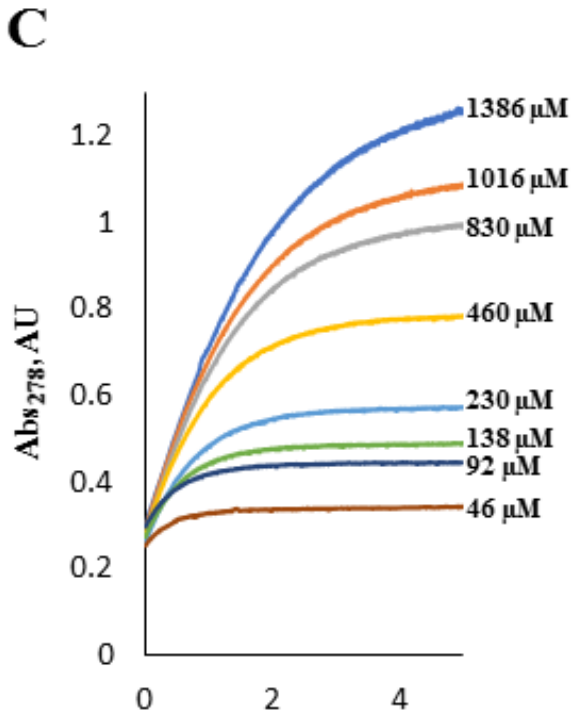

Time, $\min$
D

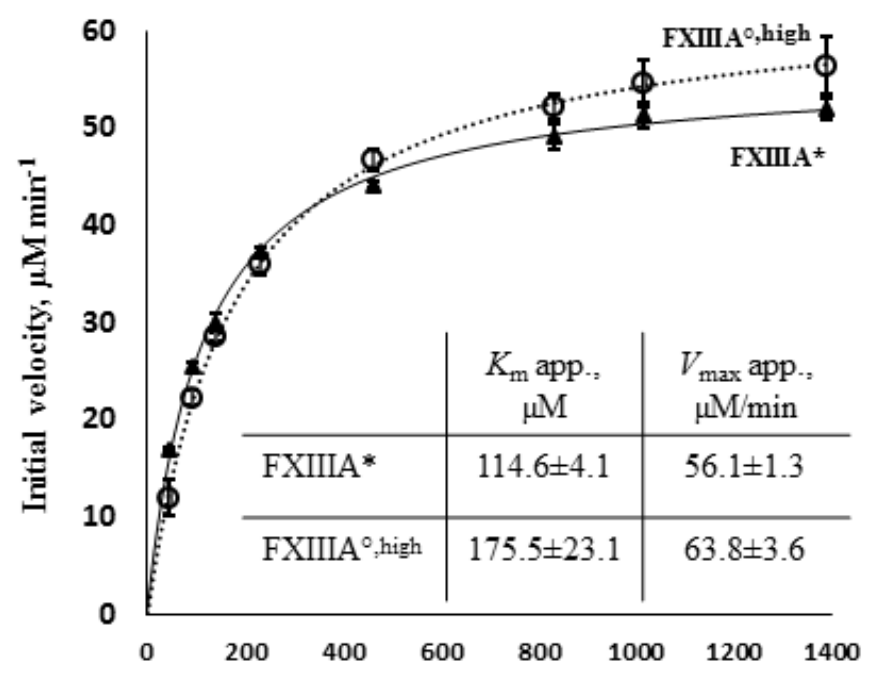

[K9], $\mu \mathrm{M}$

Fig. 31. DMPDA kinetic assay of FXIIIA transglutaminase activity. (A) A schematic of the assay reaction. (B) Representative Lineweaver-Burk plots constructed using initial velocities of crosslinking reactions in the presence of 0.5 and $1.0 \mu \mathrm{M}$ FXIIIA*. (C) Progress curves typical for the crosslinking reactions catalyzed by $1.0 \mu \mathrm{M}$ FXIIIA. (D) Michaelis-Menten fits of reaction velocities measured with $1.0 \mu \mathrm{M}$ FXIIIA* (solid line, triangles) or FXIIIA $^{\circ}$,high (dotted line, circles). Apparent $K_{\mathrm{m}}$ and $V_{\max }$ obtained from the fits are shown in the insert. Values are presented as mean $\pm \operatorname{SD}(n=3)$. 
toward the substrate would only be possible for a dimeric enzyme. As demonstrated in the current work, activated FXIIIA is a monomer. Therefore, instead of cooperative substrate binding, the currently observed effect could result from hysteretic kinetics (135) of the FXIIIA-catalyzed reaction. More precisely, a slow conformational rearrangement in the enzyme molecule would limit the overall catalytic rate. Thus, the reaction velocity would not increase anymore despite increasing substrate concentrations. Examples of such ratelimiting transitions in 'cooperative' enzymes include among others transient selfassociation or dissociation (135). Interestingly, at higher FXIIIA concentration, the negative cooperativity effect disappeared, as indicated by the absence of curvature on double-reciprocal plot (Fig. 31B, right), consistent with a possible transient dimerization of the A-subunits during the course of the catalyzed reaction. That is, higher FXIIIA concentration could accelerate hypothetical transient reassociation of the A-subunits, and such reassociation would not be rate-limiting any longer.

As another explanation, the observed negative cooperativity effect could result from limiting concentration of one of the two transglutaminase substrates. Indeed, due to decreased water solubility, a concentrated 100 mM DMPDA stock solution was prepared in methanol. Care then had to be taken in selection of the DMPDA concentration used in the assay, in order not to introduce a large amount of methanol. In addition, DMPDA in aqueous solution strongly absorbed at $220-260 \mathrm{~nm}$, resulting in the 'tale' absorbance at the product detection wavelength of $278 \mathrm{~nm}$, and thus limiting the DMPDA amount that could be employed in the assay without exceeding the linearity of absorbance readings. With the above considerations, $700 \mu \mathrm{M}$ DMPDA $(0.7 \%(\mathrm{v} / \mathrm{v})$ of methanol) as a final assay concentration was found to be optimal. Since up to $\sim 1400 \mu \mathrm{M}$ K9 was employed in the 
assay, the DMPDA would become a limiting substrate at K9 concentrations above $700 \mu \mathrm{M}$. However, as seen on Fig. 31D, the reaction velocities only approached saturation at K9 concentrations above $1 \mathrm{mM}$. In addition, inspection of the reaction progress curves revealed the maximal product accumulation of approximately $120 \mu \mathrm{M}$, thus further suggesting that reaction velocities were not simply limited by availability of DMPDA. Interestingly, a 5 min incubation of enzyme with the DMPDA (a valuable suggestion from Dr. Eugene Mueller) prior to addition of the K9 resulted in significant loss of FXIIIA transglutaminase activity (data not shown). Such inactivation of the enzyme did not occur immediately, as the initial velocities, measured over the first $20-30 \mathrm{sec}$ of the reaction, still increased with increasing K9 concentrations. Nevertheless, no affirmative conclusions could be made regarding the presence of cooperativity in FXIIIA-mediated catalysis. In addition, the $K_{\mathrm{m}}$ and $V_{\max }$ parameters obtained from the Michaelis-Menten fit are likely not the true values, and thus only serve for functional comparison of nonproteolytically and proteolytically activated FXIIIA forms.

Compared to FXIIIA ${ }^{\text {ohigh }}$, an apparent $K_{\mathrm{m}}$ for K9 peptide was almost two-fold lower for FXIIIA*, suggesting better interaction of this enzymatic form with the glutamine substrate. On the other hand, FXIII ${ }^{\circ}$,high demonstrated a stronger apparent $V_{\max }$ value suggesting that once saturated with the glutamine donor $\mathrm{K} 9$, FXIII ${ }^{\circ}$,high provides faster substrate turnover than FXIIIA*. It is necessary to note that the higher apparent $K_{\mathrm{m}}$ of the FXIII $^{\circ}{ }^{\text {,high }}$ is not simply a reflection of higher achievable velocities of this form. While at lower K9 concentrations, FXIII ${ }^{\circ \text {,high }}$ reaction velocities were lower than those for FXIIIA*, an opposite trend was observed at higher K9 concentrations. Even though the observed kinetic parameters would not result in a dramatic difference between overall catalytic 
efficiencies of the two FXIIIA forms acting on a short peptide substrate, they further corroborate proposed conformational differences between FXIIIA* and FXIIIA ${ }^{\circ}$,high .

Next, a series of SDS-PAGE assays was conducted to monitor FXIIIA-catalyzed crosslinking of a lysine mimic monodansylcadaverine (MDC) to a glutamine donor fibrinogen $\alpha \mathrm{C}$ (233-425) (Fig. 32A). The dansyl moiety of MDC provides a 'fluorescent tag' on the protein and once the $\alpha \mathrm{C}$ band is resolved by SDS-PAGE, the crosslinking reaction can be monitored by fluorescence of that band. $\alpha \mathrm{C}$ represents a physiological protein substrate of FXIIIA* and contains three reactive glutamines (136). While four lysine residues are present in the $\alpha \mathrm{C}(233-425)$ sequence, no competing $\alpha \mathrm{C}-\alpha \mathrm{C}$ conjugation, that would result in appearance of species of $\geq 40 \mathrm{kDa}$, was detected even at high $\alpha \mathrm{C}$ concentrations (Fig. 32B, bottom panel). At lower 2-10 $\mu \mathrm{M} \alpha \mathrm{C}$ concentrations, the extent of MDC incorporation catalyzed by FXIIIA* was greater than by FXIIIA ${ }^{\circ \text {,high }}$ (Fig. 32B). This difference became less apparent at $20 \mu \mathrm{M}$ and indistinguishable at the high 30-40 $\mu \mathrm{M} \alpha \mathrm{C}$ (Fig. 32B). Thus, the results obtained with a physiological protein $\alpha \mathrm{C}$ substrate indicate that FXIIIA* interacts with the glutamine donor more readily than does FXIIIA $^{\text {o,high }}$, further supporting observations with the short K9-peptide in the DMPDA assay.

To examine a possible effect of the presence of $100 \mathrm{mM} \mathrm{Ca}^{2+}$ on the $\alpha \mathrm{C}$ conformation or MDC fluorescence, the crosslinking reaction was repeated in the presence of lower $25 \mathrm{mM} \mathrm{Ca}^{2+}$ (Fig. 32C). MDC crosslinking to $\alpha \mathrm{C}$ by $100 \mathrm{mM} \mathrm{Ca}^{2+}$-activated FXIIIA ${ }^{\circ \text {,high }}$ was essentially the same regardless of $\mathrm{Ca}^{2+}$ concentration in the crosslinking reaction mix and still lower than that catalyzed by FXIIIA*. 
A
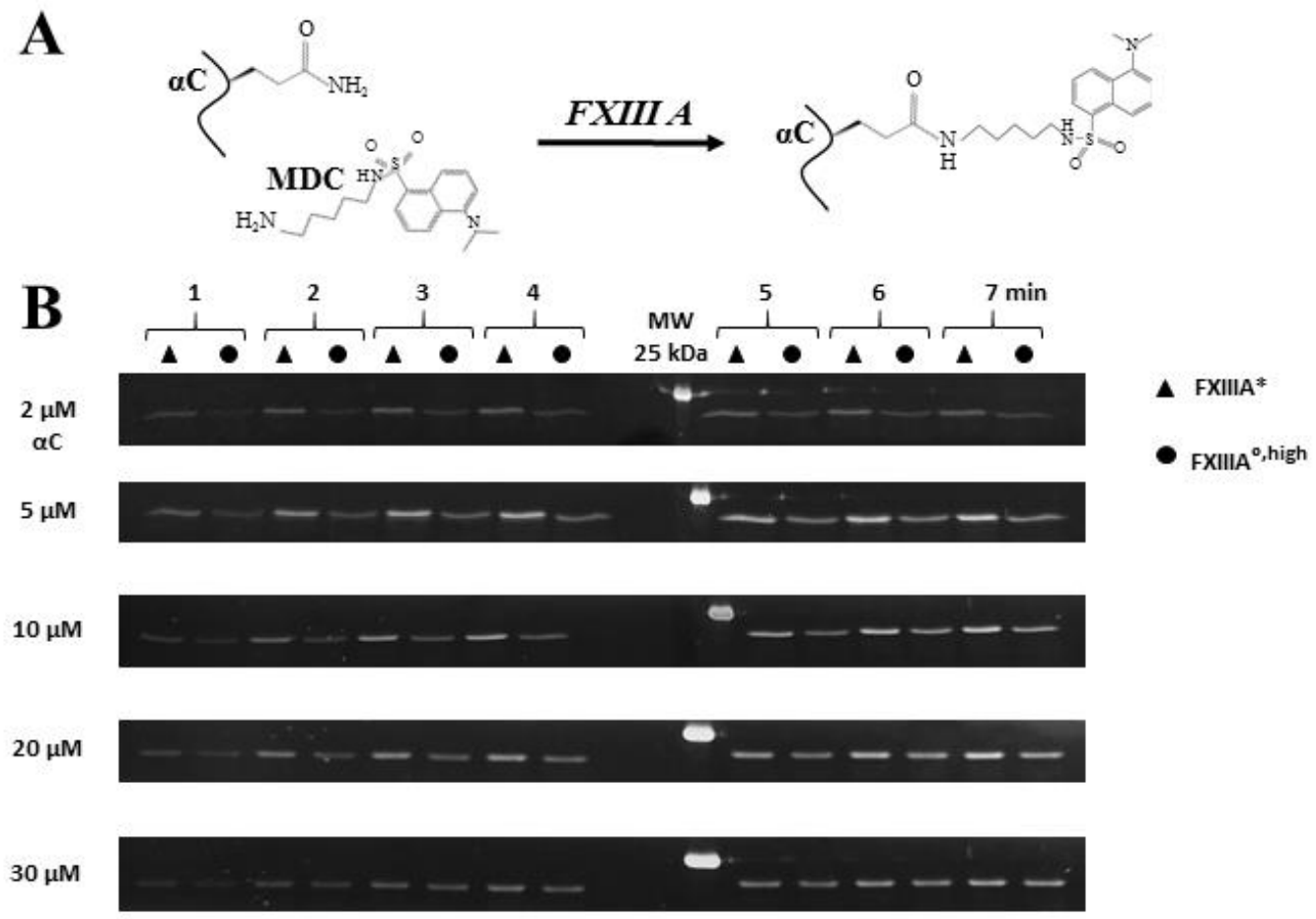

$40 \mu \mathrm{M}$
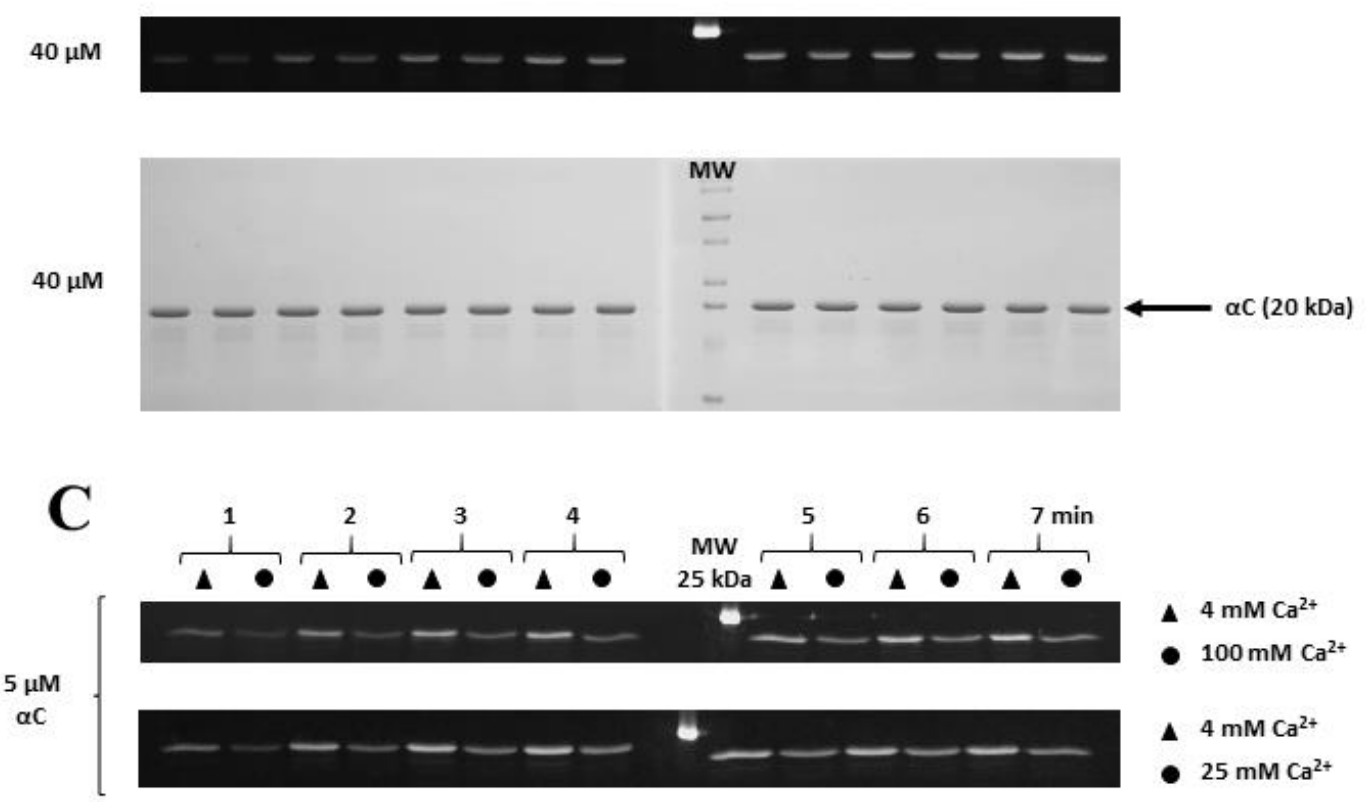

Fig. 32. (A) A schematic of the assay reaction (B) MDC assay of FXIIIA transglutaminase activity as function of $\alpha \mathrm{C}$ concentration. The samples of each reaction time point were loaded on $15 \%$ SDS-PAGE side by side for FXIIIA* (triangles) and FXIIIA ${ }^{\circ \text {,high }}$ (circles). Samples were separated on two gels: 1-4 and 5-7 min points. The gels were then aligned, transilluminated with UV, and photographed (light on dark panels). The bottom panel depicts a Coomassie Blue-stained gel pair with no competitive $\alpha \mathrm{C}-\alpha \mathrm{C}$ crosslinking. (C) MDC assay of FXIIIA ${ }^{\circ \text {,high }}$ transglutaminase activity as function of $\mathrm{CaCl}_{2}$ concentration. The layout is the same as in part A. FXIIIA ${ }^{\circ \text {,high }}$ (circles) was always activated in the presence of $100 \mathrm{mM}$ $\mathrm{CaCl}_{2}$. A control AUC experiment revealed that upon dilution to $25 \mathrm{mM} \mathrm{CaCl}_{2}, \mathrm{FXIIIA}^{\circ \text {,high }}$ remained monomeric. Samples of FXIIIA*-catalyzed reactions (triangles) are presented for comparison. 


\section{Discussion}

Currently available anticoagulants inhibit coagulation factors upstream from FXIIIA, sometimes resulting in coagulation deficiencies and unfortunate side effects ranging from increased bruising to life-threatening uncontrollable bleeding with no effective antidotes available. Being the final player of the coagulation cascade, FXIIIA has been recognized as a promising target for novel anticoagulant agents with a prospect of fewer side effects (17). Attempts are underway to design effective inhibitors of FXIIIA $(72,137,138)$. However while implementing new coagulation management regimes, it may be desirable not to affect FXIII function in nonhemostatic events. Notably, those nonhemostatic functions may themselves present therapeutic interest.

The previous chapter results qualitatively demonstrated dissociation of FXIII Asubunits during activation. The diversity of FXIIIA activation conditions in physiological compartments such as blood plasma, intracellular, and bone ECM, may impart distinct properties on the active FXIIIA forms presenting a basis for differential therapeutic targeting of FXIIIA in those compartments. This hypothesis warranted further investigation of the dynamic and functional implications of FXIIIA activation pathways. As a result, research focused on comparing FXIIIA activation by thrombin in the presence of low $\mathrm{mM} \mathrm{Ca}^{2+}$ (occurring in plasma, FXIIIA*), nonproteolytic activation by low $\mathrm{mM}$ $\mathrm{Ca}^{2+}$ (intracellular activation, FXIIIA $\left.{ }^{\circ \text { low }}\right)$, and activation in the presence of high $(\geq 25 \mathrm{mM})$ $\mathrm{Ca}^{2+}$ (bone ECM, FXIIIA $\left.{ }^{\circ, \text { high }}\right)$.

Thrombin-catalyzed proteolysis results in FXIIIA activation within minutes. Previous activity studies by Hornyak et al. (14) and Bishop et al. (105) revealed that cleavage of a single activation peptide on dimeric FXIIIA zymogen already resulted in 
expression of full activity for both, cleaved and noncleaved A-subunits. In the present study, samples where half of the FXIIIA subunits were cleaved by thrombin exhibited $\sim 3 / 4$ activity compared to the 'fully-cleaved' FXIIIA, in agreement with previously published data. Intuitively, one would expect $\sim 75 \%$ monomers in such a sample if all 'singlecleaved' species became monomers. Yet the AUC analysis revealed $62 \%$ monomeric protein, thus indicating that some of the 'single-cleaved' species remained dimeric at the concentrations and solution conditions employed in this work. In activity assays, dissociation of these species could be promoted by the presence of substrate. These results further confirm high efficiency of FXIIIA proteolytic activation. At the same time, proteolytically activated FXIIIA* tends to aggregate, suggesting a relatively short lifetime for this species.

Intracellular FXIIIA has been reported to undergo non-proteolytic activation by $\mathrm{Ca}^{2+}$ ions $(62,64)$. To imitate this activation pathway in the current work, FXIIIA was treated with low $4 \mathrm{mM} \mathrm{Ca}^{2+}$ resulting in progressive dissociation of the zymogenic dimers. Similar to FXIIIA* species, the aggregation propensity of FXIIIA ${ }^{\circ \text { low }}$ predicts its short lifetime. Such observation is in a good agreement with a study by Muszbek et al. (62), reporting only a small $6.5 \%$ of FXIIIA population within platelets being active. The slow appearance of FXIIIA ${ }^{\circ \text { low }}$ has limited its isolation and characterization in the current study. The poor solubility may suggest some similarity of this form with FXIIIA*. Furthermore, equal activity of both cleaved (FXIIIA*) and noncleaved (essentially FXIIIA ${ }^{\circ \text {,low }}$ ) subunits in the presence of low $\mathrm{mM} \mathrm{Ca}^{2+}$ supports this proposal.

In contrast to FXIIIA* and FXIIIA ${ }^{\circ \text {,low }}$, activation in the presence of high $(\geq 25 \mathrm{mM})$ $\mathrm{Ca}^{2+}$, that may proceed in ECM of the bone tissue, did not result in aggregation of 
FXIIIA ${ }^{\circ, \text { high }}$ species. Increased $\mathrm{Ca}^{2+}$ concentration was shown before to stabilize active FXIIIA in solution (14), and in current AUC experiments, higher $\mathrm{Ca}^{2+}$ levels were correlated with reduced conformational heterogeneity of FXIIIA ${ }^{\text {o, high }}$ as compared to FXIIIA*. Notably, this effect was not observed in the presence of control $\mathrm{Mg}^{2+}$. Thus, the differences in solution behavior of FXIIIA in the presence of low and high $\mathrm{mM} \mathrm{Ca}^{2+}$ imply binding of different amounts of $\mathrm{Ca}^{2+}$ to FXIIIA at different ambient concentrations of this cation. Interestingly, in the presence of low $\mathrm{mM} \mathrm{Ca}^{2+}$, activated Y283C FXIIIA* (Chapter 4), had significantly lower sedimentation coefficient than the WT FXIIIA* under the same conditions. Given the same molecular weight for WT and mutant, the decrease in $s$-value indicated a greater hydrodynamic volume for the Y283C mutant protein molecule resulting from conformational distortion. The presence of high $\mathrm{mM} \mathrm{Ca}^{2+}$ resulted in identical sedimentation properties for the mutant and WT FXIIIA, thus possibly reducing the effect of that conformational distortion.

In enzymatic evaluations, FXIIIA* had an almost two-fold lower $K_{\mathrm{m}}$ for the glutamine substrate than had FXIIIA ${ }^{\circ, \text { high }}$, thus suggesting stronger interaction of FXIIIA* with that substrate. On the other hand, FXIIIA ${ }^{\circ, \text { high }}$ provided faster reaction turnover upon saturation with the glutamine donor. It is well known that the transglutaminase reaction proceeds through a ping-pong mechanism involving binding of $\mathrm{Q}$ (glutamine) and $\mathrm{K}$ (amine) substrates (60). The possibility has been suggested of nonproductive complex formation between K-substrate and free transglutaminase enzyme (139). However, in a productive catalytic cycle, the K-substrate enters the reaction after the enzyme forms a complex with the Q-substrate. Therefore, the currently observed faster turnover by 
FXIIIA $^{\circ, \text { high }}$ may be due to better interaction with the K-substrate and faster release of the crosslinked product.

Since in the presence of low $\mathrm{mM} \mathrm{Ca}{ }^{2+}$ both cleaved and noncleaved FXIIIA monomers are equally active, as indicated in previous publications $(14,105)$ and confirmed in the current study, the observed differences in activities of FXIIIA* and FXIIIA ${ }^{\circ \text {,high }}$ could not be explained by the absence or presence of the activation peptide. Another important factor to consider is, again, the possible difference in amount of $\mathrm{Ca}^{2+}$ bound by those two FXIIIA forms. In early work, Lewis and coworkers (140) reported that each Asubunit of plasma zymogen FXIII $\mathrm{A}_{2} \mathrm{~B}_{2}$ binds a $\mathrm{Ca}^{2+}$ ion with $K_{\mathrm{D}}=100 \mu \mathrm{M}$. Hornyak et al. (82) later demonstrated that another $\mathrm{Ca}^{2+}$ ion is required by each A-subunit to dissociate from the B-subunits and to expose catalytic cysteines.

Stieler and coworkers (72) recently crystallized FXIIIA ${ }^{\circ \text {,high }}$ with a covalently bound Q-mimic inhibitor providing the first molecular structure of active FXIIIA. Besides the previously observed first, zymogenic $\mathrm{Ca}^{2+}$ site (70), the Stieler research group elegantly demonstrated the functional significance of two additional sites. In particular, development of the $2^{\text {nd }} \mathrm{Ca}^{2+}$ site brought about conformational changes in the FXIIIA molecule to initiate formation of a hydrophobic pocket for the K-substrate entrance. This site is the same as that presumed by Hornyak (82) to promote exposure of the reactive thiol on the A-subunits. Coordination of the $3^{\text {rd }} \mathrm{Ca}^{2+}$ was proposed by Stieler et al. to conclude formation of the hydrophobic pocket and to facilitate binding of the K-substrate. Since the binding of Q and $\mathrm{K}$ is sequential and the presented crystal structure resembles a covalent FXIIIA-Qsubstrate intermediate, an idea may be entertained that this $3^{\text {rd }}$ site is filled with $\mathrm{Ca}^{2+}$ upon binding or reaction of the Q-substrate, therefore optimizing entrance of the K-substrate. 
Intriguingly, the tall narrow sedimentation peaks of covalently inhibited FXIIIA* (Fig. 23, Chapter 4) were observed at low $4 \mathrm{mM} \mathrm{Ca}^{2+}$. The close resemblance of those peaks with the one for free $\mathrm{FXIII}^{\circ \text {,high }}$ enzyme may imply that $\mathrm{FXIIIA}^{*}$ did bind an additional $\mathrm{Ca}^{2+}$ ion upon inhibition with the Q-substrate mimic and adopted a conformation similar to FXIII ${ }^{\text {,high }}$.

Whether binding of $\mathrm{Ca}^{2+}$ at the $3^{\text {rd }}$ site precedes entrance of the K-substrate during the normal course of transglutaminase reaction (without inhibitors), is open to speculation. It seems plausible, however, that at relatively low $\mathrm{Ca}^{2+}$ levels, coordination of this $3^{\text {rd }}$ site and completion of the hydrophobic pocket is at least partially dependent on the availability of the K-substrate. It is known that in the absence of K-substrate, a water molecule acts as an acyl acceptor resulting in net deamidation of the glutamine to glutamate (59). The $\mathrm{Ca}^{2+}$ requirement for deamidation catalyzed by guinea pig liver transglutaminase was demonstrated to be much lower than that for transamidation (141), thus raising such a possibility for homologous FXIIIA.

In conditions where $\mathrm{Ca}^{2+}$ concentrations are relatively high, such as in bone ECM, the $3^{\text {rd }}$ FXIIIA $\mathrm{Ca}^{2+}$ site may already be saturated even in a free enzyme, prior to the Qsubstrate binding. The present kinetic results suggest that this saturation facilitates a conformation (FXIIIA ${ }^{\circ}$,high $)$ that has a weaker affinity for the Q-substrate (indicated by higher apparent $K_{\mathrm{m}}$ in the current study) but provides a mild improvement to reaction turnover compared to FXIIIA* (higher apparent $V_{\max }$ ). In addition to the stabilizing effect observed in the current study, the FXIIIA ${ }^{\circ \text {,high }}$ conformation may also favor crosslinking (transamidation) of the protein substrates over deamidation, which may be especially relevant in acidic conditions of the bone ECM. 
Overall, the presented research expands the understanding of the functional outcomes of the FXIII activation pathways. Based on the previous and current results, the following model of physiological FXIII activation can be envisioned: nonproteolytic activation proceeds relatively slow, but constantly. High $\mathrm{mM} \mathrm{Ca}{ }^{2+}$ levels, such as in bone ECM, stabilize the FXIIIA ${ }^{\text {,high }}$ form and provide its constitutive activity as part of the tissue remodeling processes. By contrast, thrombin-mediated AP-removal facilitates binding of $\mathrm{Ca}^{2+}$ even at low Ca levels, thus promoting fast build up of FXIII crosslinking activity in plasma. The resultant FXIIIA* species have high affinity towards glutamine substrates and respond even to low concentrations of these substrates, ensuring clotstabilizing function during blood coagulation. At the same time, the tendency of FXIIIA* to aggregation may determine its relatively short life time in plasma, providing an efficient way to prevent detrimental excessive protein crosslinking. Different activation conditions also result in conformationally different FXIIIA forms thus providing a possible basis for developing therapeutic strategies for compartment-specific management of FXIIIA function. $^{1}$

\footnotetext{
${ }^{1}$ Some contents of this chapter are reproduced from the published manuscript: Anokhin, B.A., Stribinskis, V., Dean, W.L., and Maurer, M.C. (2017) Activation of factor XIII is accompanied by a change in oligomerization state. FEBS J 284, 3849-3861.
} 


\section{CHAPTER 6}

\section{STUDYING THE EFFECT OF FXIIIA ACTIVATION RATE ON FIBRIN CLOT ARCHITECTURE}

\section{Introduction}

The three key players at the final stage of blood coagulation that participate in formation of a blood clot include fibrinogen, thrombin, and FXIII. Fibrinogen, a dimer of trimers $(\mathrm{A} \alpha \mathrm{B} \beta \gamma)_{2}$, undergoes thrombin mediated cleavage of the $\mathrm{N}$-terminal fibrinopeptides $\mathrm{A}$ and $\mathrm{B}$ from the $\mathrm{A} \alpha$ and $\mathrm{B} \beta$ chains. The resulting fibrin self-polymerizes to create a fibrin network $(11,12)$. Simultaneous with cleavage of fibrinogen, FXIIIA is subjected to proteolytic removal of its activation peptides by thrombin, thus converting to an active transglutaminase that introduces covalent crosslinks within the fibrin network $(2,4)$.

The common L34 polymorphism in the FXIII AP is found in about $25 \%$ of the Caucasian population and has been associated with faster activation of FXIII by thrombin than the more prevalent V34 $(88,90,142,143)$. Kinetic studies with synthetic FXIII activation peptide sequences revealed that the extra methylene group in FXIII AP L34 generates a thrombin substrate with a strong enhancement in $k_{\text {cat }}(90,94)$. Additional experiments further demonstrated the critical role that the 34 position can play in controlling thrombin-catalyzed hydrolysis of the FXIII activation peptide segment (9193,96). These experiments resulted in the proposal that the fibrin clot properties could be 
controlled by designing FXIII activation peptide sequences that are easier or more difficult to be cleaved by thrombin. The current work has focused on examining the effect of FXIIIA proteolytic activation rate on fibrin clot structure.

\section{Materials and Methods}

\section{Materials}

Recombinant human thrombin was generously provided by Dr. Enrico Di Cera and Dr. Leslie Pelc (St. Louis University). Bovine thrombin was purchased from SigmaAldrich. Human FXIII-free peak 1 fibrinogen was purchased from Enzyme Research Laboratories (South Bend, IN). Plasma from FXIIIA-deficient mice was kindly provided by Dr. Matthew Flick (Cincinnati Children's Hospital, Cincinnati, OH). Thrombin inhibitor PPACK was from Haematologic Technologies (Essex Junction, VT). FXIII AP variants were recombinantly expressed and purified as described in Chapter 3.

\section{Thrombin-catalyzed cleavage of FXIIIA AP variants}

Cleavage of FXIII AP variants was monitored using SDS-PAGE. $1 \mu \mathrm{M}$ FXIII V34X variant was incubated in TBS (50 mM Tris Acetate, $\mathrm{pH} 7.4,150 \mathrm{mM} \mathrm{NaCl})$ with $30 \mathrm{nM}$ of recombinant human thrombin (WT or I174A) at $37^{\circ} \mathrm{C}$. At different time points $(1,2,5,10,15,20,25$, and $30 \mathrm{~min})$, aliquots were withdrawn and supplemented with $6 \mu \mathrm{M}$ PPACK. Samples were then mixed with reducing loading buffer and subjected to SDSPAGE using $8 \%$ polyacrylamide gels. The cleavage process was monitored by densitometric analysis using GelAnalyzer 2010 (www.gelanalyzer.com). 
Fibrin crosslinking in the presence of FXIIIA AP variants

Human FXIII-free fibrinogen (1 mg/ml), a recombinant FXIIIA AP variant (V34, L34, F34, or W34, $50 \mathrm{nM})$, and recombinant WT human thrombin (12 nM) were incubated in TBS in the presence of $2.5 \mathrm{mM} \mathrm{CaCl} 2$ at $37^{\circ} \mathrm{C}$. At each time point $(5-60 \mathrm{~min})$, the reaction was quenched by addition of reducing loading buffer followed by boiling for 5 min, resulting in full solubilization of the formed clots. Samples were then subjected to SDS-PAGE on an $8 \%$ gel.

\section{Clot turbidity assay}

In a quartz cuvette, $0.5 \mathrm{mg} / \mathrm{ml}$ FXIII-free human fibrinogen was combined with $100 \mathrm{nM}$ recombinant FXIIIA variant (V34, L34, F34, or W34) in borate buffer (20 mM boric acid, $\mathrm{pH} 7.8,150 \mathrm{mM} \mathrm{NaCl}$ ) supplemented with $2.5 \mathrm{mM} \mathrm{CaCl}_{2}$. The mixture was warmed to $37^{\circ} \mathrm{C}$, and clotting was initiated by addition of $5 \mathrm{nM}$ recombinant human thrombin (WT or I174A). Fibrin polymerization was monitored by increasing turbidity at $340 \mathrm{~nm}$. Control experiments were performed without addition of FXIIIA.

Preparation of fibrin clots for SEM

FXIII-free fibrinogen from human plasma $(0.5-2.0 \mathrm{mg} / \mathrm{ml})$ was combined with recombinant FXIIIA AP variant (L34, V34 or W34, $100 \mathrm{nM}$ ) in borate buffer. The mixture was incubated at $37^{\circ} \mathrm{C}$ for $5 \mathrm{~min}$, and the clotting was initiated by addition of recombinant human thrombin $(5-30 \mathrm{nM})$ and $\mathrm{CaCl}_{2}(2.5 \mathrm{mM})$. Control clot samples were made without addition of FXIII. Clots were formed in capped microfuge tubes for $2 \mathrm{~h}$ at $37^{\circ} \mathrm{C}$. Whole plasma clots were prepared in the same manner with the following modifications: $13.5 \mathrm{mM}$ 
$\mathrm{CaCl}_{2}$ was used instead of $2.5 \mathrm{mM}$, to exceed $10.5 \mathrm{mM}$ citrate present in plasma; and bovine thrombin at $2.1 \mathrm{NIH} \mathrm{U/ml} \mathrm{(corresponding} \mathrm{to} \mathrm{approximately} 21 \mathrm{nM}$ ) was used instead of recombinant human thrombin.

The gelated clots were rinsed with the buffer and dehydrated in a series of solutions with increasing ethanol concentrations $(10-95 \% \mathrm{v} / \mathrm{v}, 10-30 \mathrm{~min}$ in each solution, followed by three changes of $100 \%$ ethanol). The ethanol was then replaced with hexamethyldisilazane (HMDS, two changes). In some experiments, clots were fixed for 30 $\min$ in $2 \%(\mathrm{v} / \mathrm{v})$ solution of glutaraldehyde in the borate buffer prior to ethanol dehydration.

During initial preparative steps, gelated clots remained attached to the walls of microfuge tubes, and the solutions were changed by simple pipetting, avoiding contact of the clots with the pipette tip. At later stages of ethanol dehydration, clots detached from the tube walls and were suspended in the liquid. After ethanol was replaced with HMDS, clots were transferred onto aluminum stubs and left to air-dry overnight.

\section{SEM analysis of fibrin clots}

SEM of fibrin clots was performed at the University of Louisville Huson Imaging and Characterization facility. For better electron conductivity, samples were sputter-coated with gold/palladium using argon plasma at 0.1 Torr and $1.3-1.4 \mathrm{kV}$ for $3 \mathrm{~min}$ (these conditions result in deposition of a $\sim 20 \mathrm{~nm}$ metal layer). Imaging was conducted in the EVO or Supra-35 scanning electron microscope (Carl Zeiss) operating in low pressure mode using the beam accelerating voltage of $1-6 \mathrm{kV}$. 


\section{Results}

Thrombin-catalyzed cleavage of FXIIIA AP variants

In a series of experiments, recombinant FXIIIA AP variants were subjected to limited proteolysis by thrombin. In full length FXIIIA, cleavage of the activation peptide by thrombin results in a $4 \mathrm{kDa}$ molecular weight loss, thus cleaved and noncleaved Asubunits could be separated by SDS-PAGE (Fig. 33A). Using densitometric analysis of the gels, the full length FXIII AP variants were ranked in terms of their cleavage rates by WT thrombin L34>>V34>F34>>W34 (Fig. 33B, left), a trend similar to that previously obtained using synthetic activation peptide fragments $(91-93,96)$. For the I174A thrombin mutant with a modified architecture of the apolar binding site, W34 FXIIIA became the best substrate of the series (Fig. 33B, right). The rate of W34 AP cleavage by mutant thrombin was comparable with that of the V34 FXIIIA - WT thrombin pair (Fig. 33B, left), in further agreement with the synthetic peptide studies.

\section{Fibrin crosslinking by FXIIIA AP variants}

The FXIIIA L34, V34, F34, and W34 AP variants were combined with human FXIII-free fibrinogen and clotting was initiated by addition of WT thrombin and $\mathrm{Ca}^{2+}$. As expected, the rate of FXIIIA proteolytic activation determined development of the crosslinking activity (Fig. 34). The fastest cleaved FXIIIA L34 generated both $\gamma-\gamma$ and high molecular weight (HMW) fibrin species already at the early stage $(5 \mathrm{~min})$ of the experiment. Slow cleavage of FXIIIA W34 by thrombin resulted in the slowest appearance of $\gamma-\gamma$ and very little HMW crosslinks. Interestingly, F34 FXIIIA was cleaved by thrombin slightly slower than V34 (thrombin cleavage figure), however, both variants crosslinked 
A

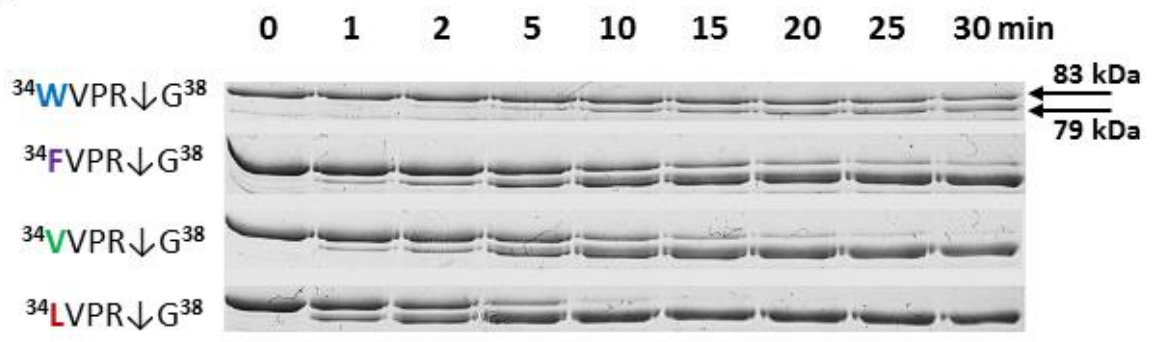

B
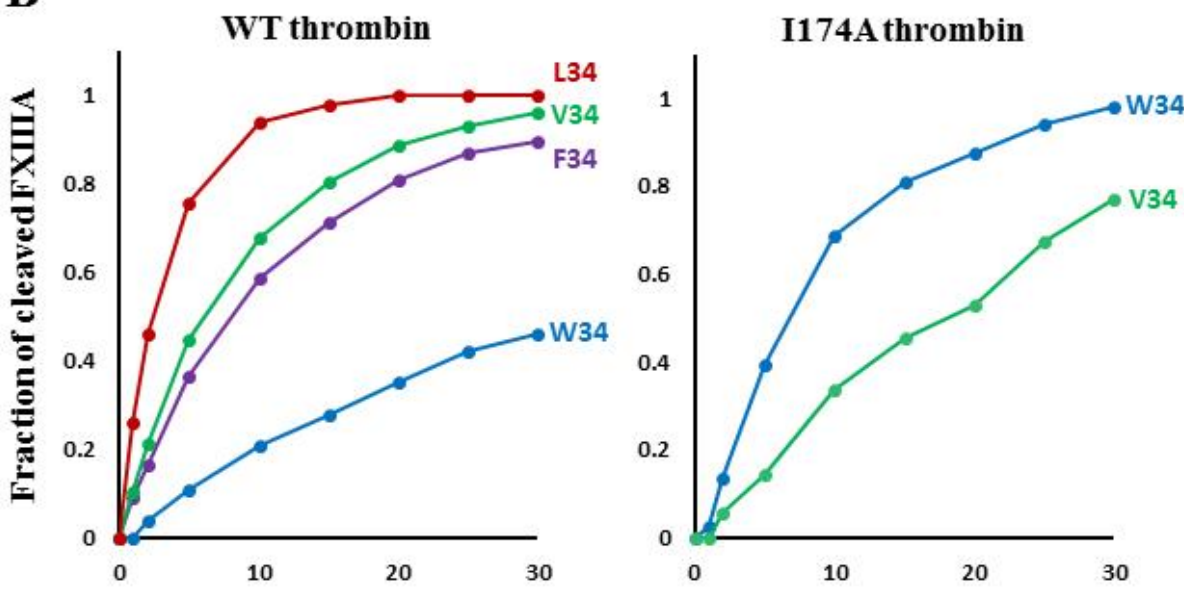

Time, $\min$

Fig. 33. Thrombin-catalyzed cleavage of full length FXIIIA. (A) Representative results of the SDS-PAGE assay to follow progress of FXIIIA AP variants cleavage by WT thrombin. FXIIIA amino acid residues of the cleavage site are depicted on the left. $\downarrow$ denotes the scissile R37-G38 bond. (B) FXIIIA AP variants cleavage progress in the presence of WT (left) and I174 mutant thrombin (right) monitored by densitometric analysis of the gels.

fibrin to a similar extent (Fig. 34). This observation may in part be explained by the fact that the cleavage of a single AP on dimeric FXIIIA zymogen promotes full activity of both cleaved and noncleaved A-subunits, as reported in previous studies (14) and described in Chapter 5 of the present dissertation. In addition, a competition between FXIIIA and fibrinogen for the thrombin active site may have further masked the difference in V34 and F34 cleavage rate observed in the absence of fibrinogen. Overall, the results demonstrate that the rate of FXIIIA activation influences the extent of covalent crosslinking in a polymerizing fibrin clot. 


\section{$\begin{array}{lllllll}0 & 5 & 10 & 15 & 30 & 45 & 60\end{array}$}

W34

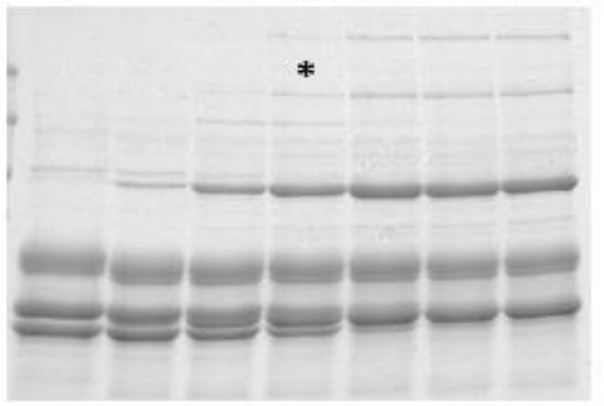

F34

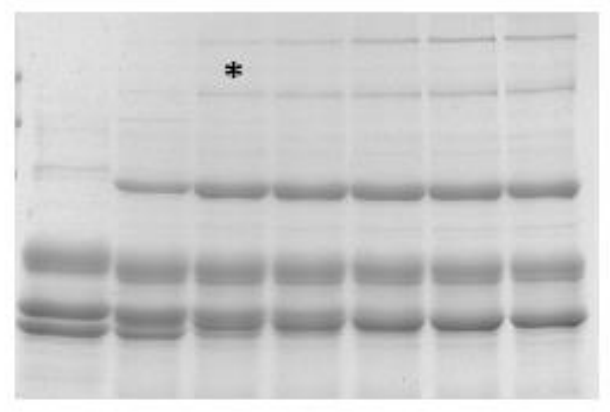

V34
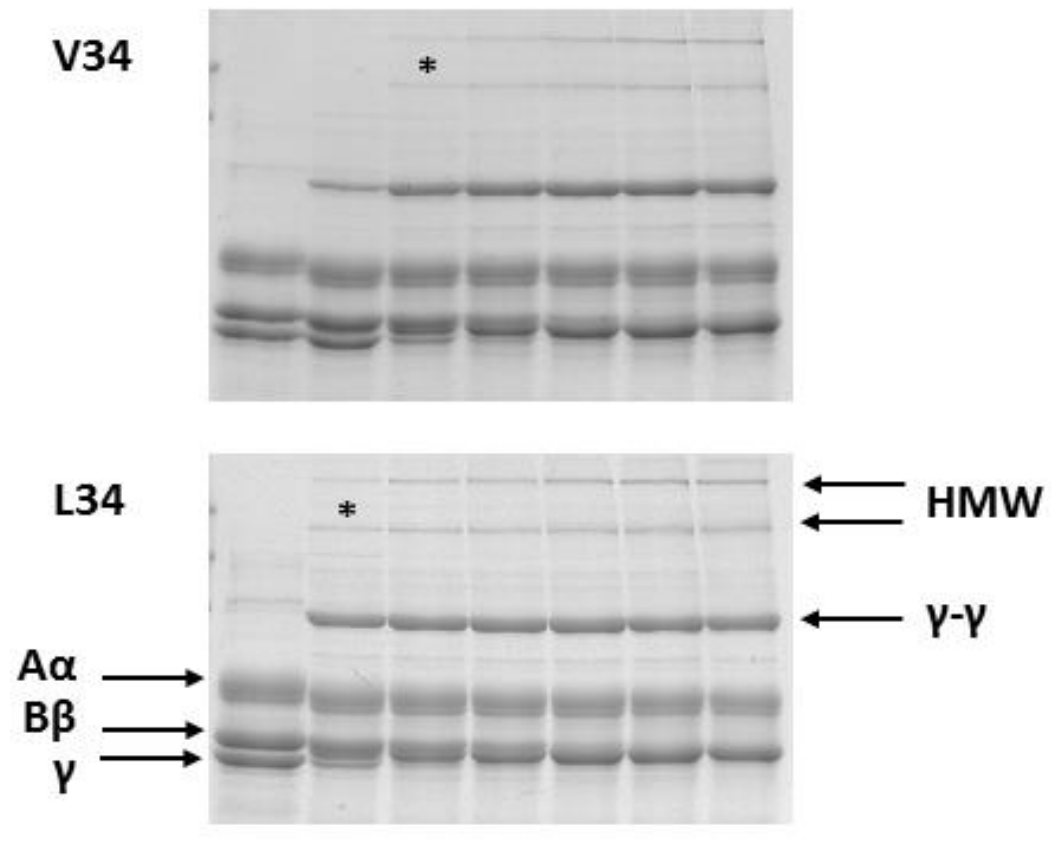

Fig. 34. An SDS-PAGE assay to follow covalent fibrin crosslinking by FXIIIA AP variants. Human FXIIIfree fibrinogen was combined with a FXIIIA AP variant, and clotting was initiated by addition of WT thrombin and $\mathrm{CaCl}_{2}$. On each gel, the " 0 " time point represents fibrinogen ( $\mathrm{A} \alpha, \mathrm{B} \beta$ and $\gamma$ chains are denoted). As thrombin cleaves fibrinopeptides A and B during conversion of fibrinogen to fibrin, the resulting fibrin $\alpha$ and $\beta$ chains have lower molecular weight than their precursors in fibrinogen. Disappearance of the $\gamma$-chains can be observed on the present gels due to their relatively fast conjugation into covalent $\gamma-\gamma$ dimers by FXIIIA. HMW - high molecular weight $\gamma-\alpha$ and $\alpha-\alpha$ hybrids are also crosslinked by FXIIIA. * denotes the earliest detection of HMW species during the course of the crosslinking reaction. 


\section{Monitoring turbidity of fibrin clots}

Fibrin polymerization in the presence of WT thrombin, with and without the presence of FXIIIA AP variants, was monitored photometrically by increasing turbidity at $340 \mathrm{~nm}$. The following parameters were assessed: (i) the lag phase duration, reflecting time necessary for thrombin to convert fibrinogen to fibrin in quantities sufficient for polymerization, (ii) polymerization rate, indicated by the velocity of absorbance increase due to formation of large supramolecular fibrin aggregates (fibers), and (iii) maximal absorbance at plateau, conventionally correlated with the thickness of the fibers.

In the absence of FXIIIA, the lag time before fibrin polymerization was $45 \mathrm{sec}$ (Table 4). This lag was extended in the presence of FXIIIA variants, consistent with the competition between FXIIIA and fibrinogen for thrombin. The polymerization rates, estimated from the slope of turbidity increase, and the final turbidity values were slightly lower in the presence of FXIIIA (Fig. 35, Table 4). However, no clear dependence of fibrin polymerization on the FXIIIA activation rate was observed.

In the presence of I174A thrombin with decreased ability to cleave fibrinogen, the fibrin formation and gelation proceeded significantly slower than with WT thrombin (Fig. 35, right, and Table). The lag-time approached $9 \mathrm{~min}$, and compared to the WT thrombin, fibrin polymerization rate observed with I174A thrombin was significantly reduced. Interestingly, the presence of FXIIIA had now a more pronounced effect on the fibrin polymerization resulting in significantly lower achievable turbidity values. The extent of this effect was clearly dependent on the FXIIIA activation rate: turbidity value was lowest in the presence of faster-activated W34 FXIIIA, increased in the presence of slower-activated V34 FXIIIA, and was maximal in the absence of FXIIIA. 


\section{WT thrombin}

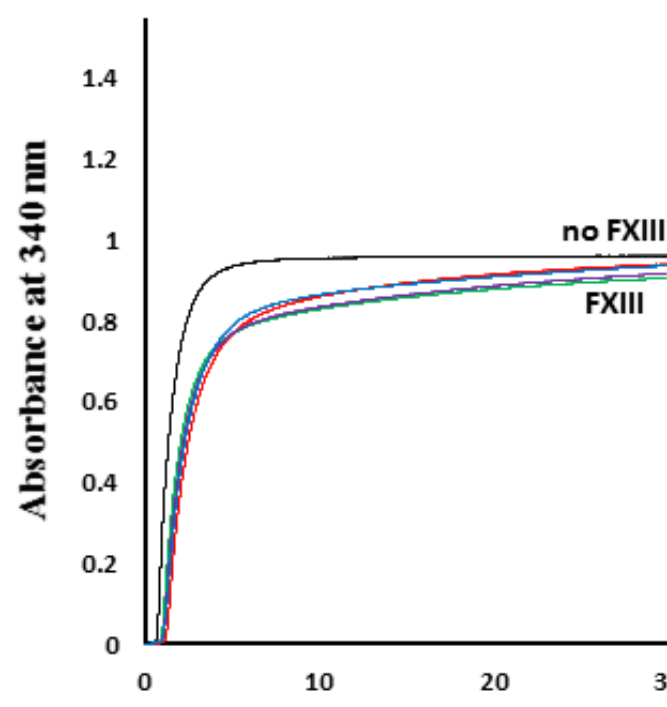

I174A thrombin

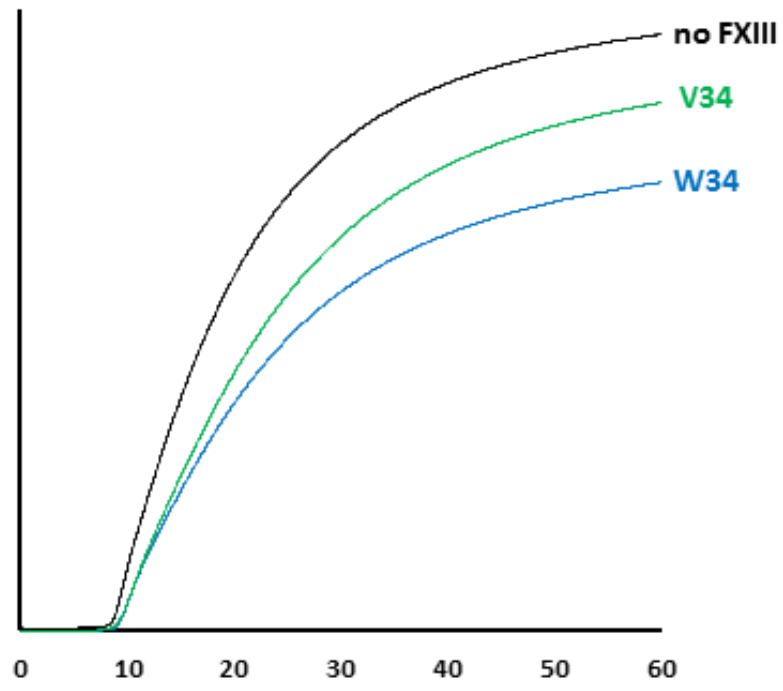

Time, $\min$

Fig. 35. Fibrin turbidity assay. Human FXIII-free fibrinogen was combined with a FXIIIA AP variant in a spectrophotometric cuvette, and the clotting was initiated by addition of WT (left) or I174A thrombin mutant (right) and $\mathrm{CaCl}_{2}$. Fibrin polymerization was monitored by increasing absorbance at $340 \mathrm{~nm}$. Colored curves represent fibrin turbidity in the presence of the FXIII AP-variants, black curves represent control experiments without addition of FXIIIA. Note the different duration of experiments with WT (30 $\mathrm{min}$ ) and I174A thrombin (60 $\mathrm{min})$.

Table 4. Fibrin turbidity parameters.

\begin{tabular}{|c|c|c|c|}
\hline FXIIIA variant & lag duration, min & $\begin{array}{c}\text { Initial } \\
\text { polymerization } \\
\text { rate, } \mathrm{AU} / \mathrm{min}\end{array}$ & $\begin{array}{c}\text { Absorbance at } \\
\text { plateau, AU }\end{array}$ \\
\hline \multicolumn{4}{|c|}{ WT thrombin } \\
\hline No FXIIIA & 0.75 & 0.90 & 0.96 \\
\hline W34 & 1.14 & 0.50 & 0.93 \\
\hline F34 & 1.13 & 0.52 & 0.92 \\
\hline V34 & 1.05 & 0.62 & 0.90 \\
\hline L34 & 1.30 & 0.52 & 0.94 \\
\hline \multicolumn{4}{|c|}{ I174A thrombin } \\
\hline No FXIIIA & 8.60 & 0.10 & 1.50 \\
\hline W34 & 8.90 & 0.07 & 1.13 \\
\hline V34 & 8.90 & 0.07 & 1.40 \\
\hline
\end{tabular}




\section{SEM analysis of clots formed with purified human fibrinogen}

To study the possible effect of FXIIIA crosslinking on the thickness of fibrin fibers, FXIIIA variants with different activation rates were combined with FXIII-free fibrinogen purified from human plasma. The clotting was initiated by addition of thrombin and $\mathrm{CaCl}_{2}$. In initial experiments, clots were fixed with $2 \%$ glutaraldehyde upon gelation, followed by stepwise dehydration with 50, 70, 90, 95, and 100\% ethanol, and drying with HMDS. Clots were sputtered with gold/palladium and imaged in a Zeiss EVO electron microscope at the beam accelerating voltage of $6 \mathrm{kV}$. The major area of the clot surface was found to be covered with a 'coating', and the fiber-resembling structures could barely be observed underneath (Fig. 36A). A limited number of coating-free spots were present revealing the characteristic network of fibrin fibers (Fig. 36B). Excessive charging, however, prevented acquisition of clot images of good quality. Since the clots were mounted on to aluminum stubs before deposition of gold-palladium, the conductive layer would effectively couple the surface area of the clot with the stub and consequently, with the grounded specimen stage. Therefore, the charge was likely accumulating relatively far below the surface of the samples. Reducing beam-sample interaction volume by lowering the accelerating voltage helped to minimize the charging effect; however, it led to increased noise.

Another instrument, the Zeiss Supra, is a field emission SEM that generates a narrower electron beam even at low accelerating voltages. Using this instrument, it was possible to obtain clot images of better quality at low $1-3 \mathrm{kV}$ without significant charge accumulation. Still, the coating observed on the surface of the clots limited the number of areas with exposed fibers (Fig. 36C). At higher magnification (Fig. 36D), the fibrin fibers could be seen 'stemming' from the coating suggesting that non-polymerized fibrin 
A

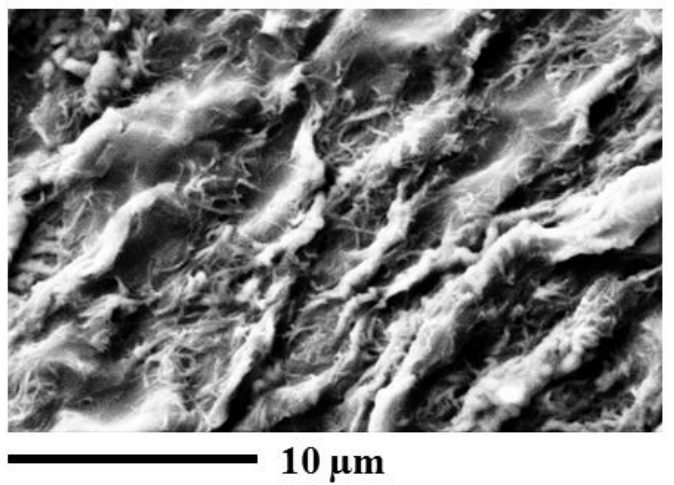

C

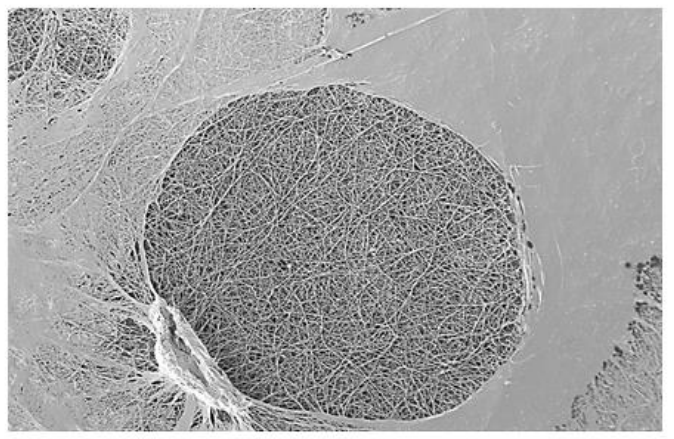

$50 \mu \mathrm{m}$

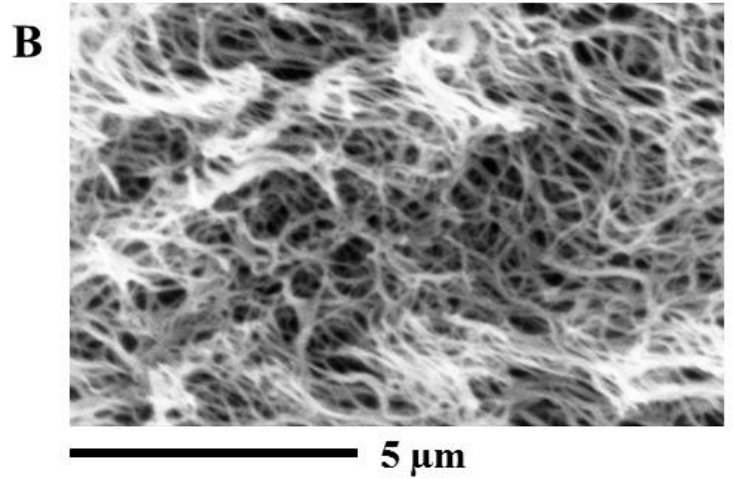

D

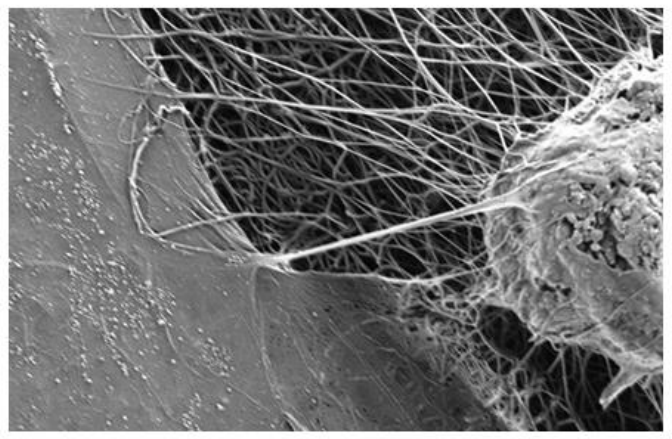

$10 \mu \mathrm{m}$

Fig. 36. SEM analysis of fibrin clots fixed with glutaraldehyde. (A) A typical view of a fibrin clot surface with a coating. (B) Coating-free area of the clot revealing the fibrin network. Images A and B were obtained on the Zeiss Evo at $6 \mathrm{kV}$. Excessively bright spots on both images and the lack of focus at higher magnification (B) resulted from accumulation of charge on the specimen. (C) An image obtained using a thinner clot sample and at a lower $(1 \mathrm{kV})$ accelerating voltage in the Zeiss Supra FESEM, with the coating still present.

stabilized by glutaraldehyde crosslinking might constitute that coating. Instead of using glutaraldehyde to preserve the fiber structure, the dehydration procedure was adjusted to include a smoother ethanol gradient: 10, 20, 30 50, 70, 90, and 100\%. As a result, no coating was observed, and surprisingly, elimination of glutaraldehyde from the sample preparation did not affect the morphology of fibrin fibers.

Qualitatively similar clot architectures were observed through an entire range of employed fibrinogen $(0.5,1.0$, and $2.0 \mathrm{mg} / \mathrm{ml})$ and thrombin $(5,15$, and $30 \mathrm{nM})$ concentrations (Appendices 1-4). In general, the fibrin clots were comprised of thin and 
A

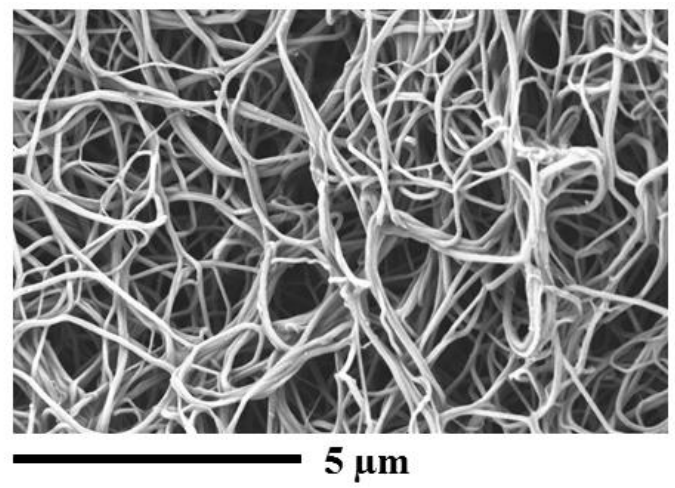

$\mathrm{C}$

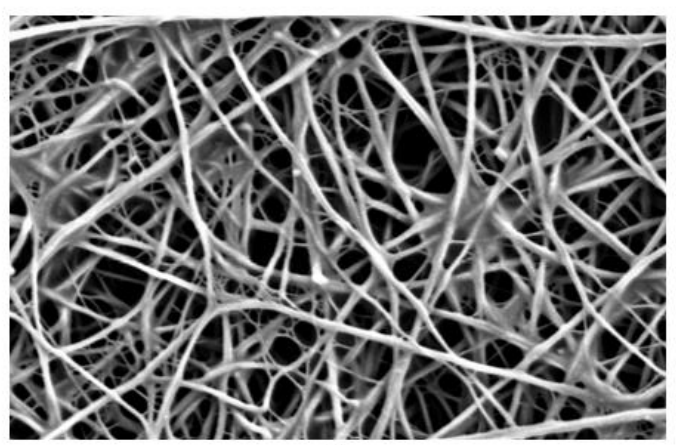

$5 \mu \mathrm{m}$
B

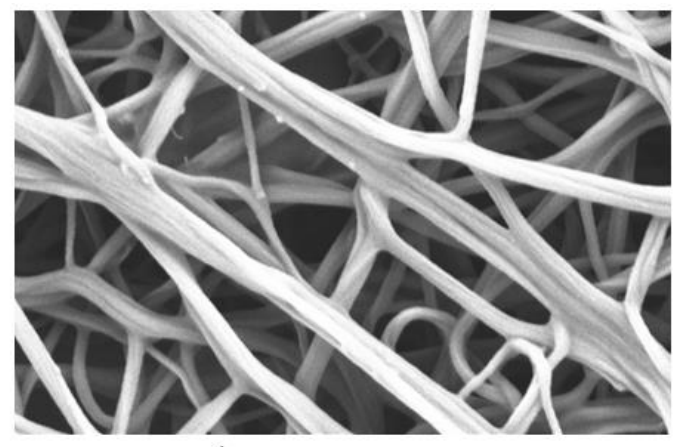

$1 \mu \mathrm{m}$

D

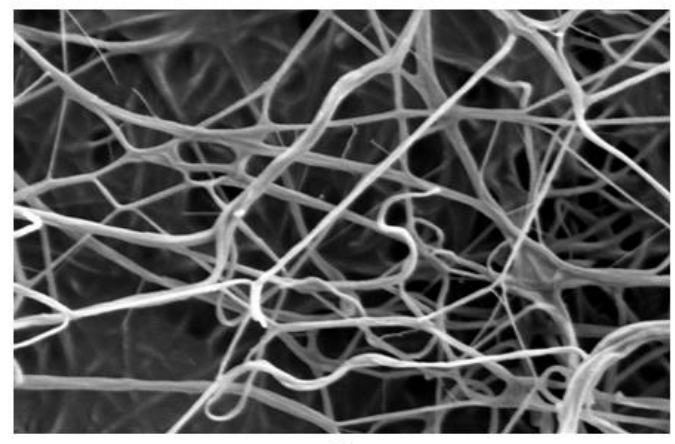

$5 \mu \mathrm{m}$

Fig. 37. SEM analysis of fibrin clots. (A) and (B) - representative images of fibrin clots formed in the presence of FXIIIA AP variants and WT thrombin. (C) and (D) - fibrin clots formed in the presence of I174 mutant thrombin and FXIIIA AP variants V34 (C) and W34 (D). Clots in images C and D were fixed with glutaraldehyde.

thick fibers (Fig. 37A). More detailed examination at higher magnifications revealed that thick fibers resulted from fusion of the thinner fibers (Fig. 37B). Neither the thickness of individual fibers nor the amount of fused fibers correlated with the extent of covalent fibrin crosslinking introduced by FXIIIA AP variants. Ironically, major differences between the V34X clots were global in nature and were observed during the sample preparation process. In particular, the ability to retain original clot volume during dehydration was associated with the extent of FXIII-catalyzed crosslinking and increased in order [FXIII-free clots] < [W34-clots $]<$ [V34-clots] $<$ [L34-clots]. That is, the clots with no or minimal crosslinking reduced in volume already in the presence of low concentrations of ethanol. By contrast, 
the most crosslinked clots underwent compaction only at higher ethanol concentrations. Thereby, the tolerance to the presence of increasing ethanol concentrations indicated the different strengths of the fibrin networks crosslinked by FXIIIA AP variants. Clots formed in the presence of thrombin mutant I174A demonstrated a similar dependence of native volume retention on the extent of FXIIIA-catalyzed crosslinking: W34 FXIIIA was activated by I174A thrombin faster than V34, and the W34-crosslinked clot was mechanically stronger. The fiber thickness of I174A-clots (Fig. 37C and D) studied by SEM was similar to that of the clots formed with WT thrombin.

\section{SEM of clots formed with whole plasma}

Since no FXIIIA-related differences in fiber thickness and overall clot architectures were observed in a pure protein system, there was an interest to study clot morphology in a more physiological whole plasma environment. The first whole clots were made using plasma from mice with normal FXIII phenotype. Those clots were relatively thick and, similarly to the pure fibrin clots, underwent charging in the SEM. Cutting the clots into thinner sections alleviated the charging problem but resulted in artificial smashing of the fibers in some areas (Fig. 38A). In addition, the excessively dense fiber network complicated morphological evaluation. A 1:4 dilution of the whole murine plasma resulted in thinner clots that did not require dissection and had optimal network density (Fig. 38B).

Next, recombinant FXIIIA V34X AP variants were administered into the plasma from FXIIIA-null mice. Clots formed without FXIIIA significantly 'flattened' during the dehydration process, and thus fibrin fibers on the SEM images (Fig. 39) appear denser 

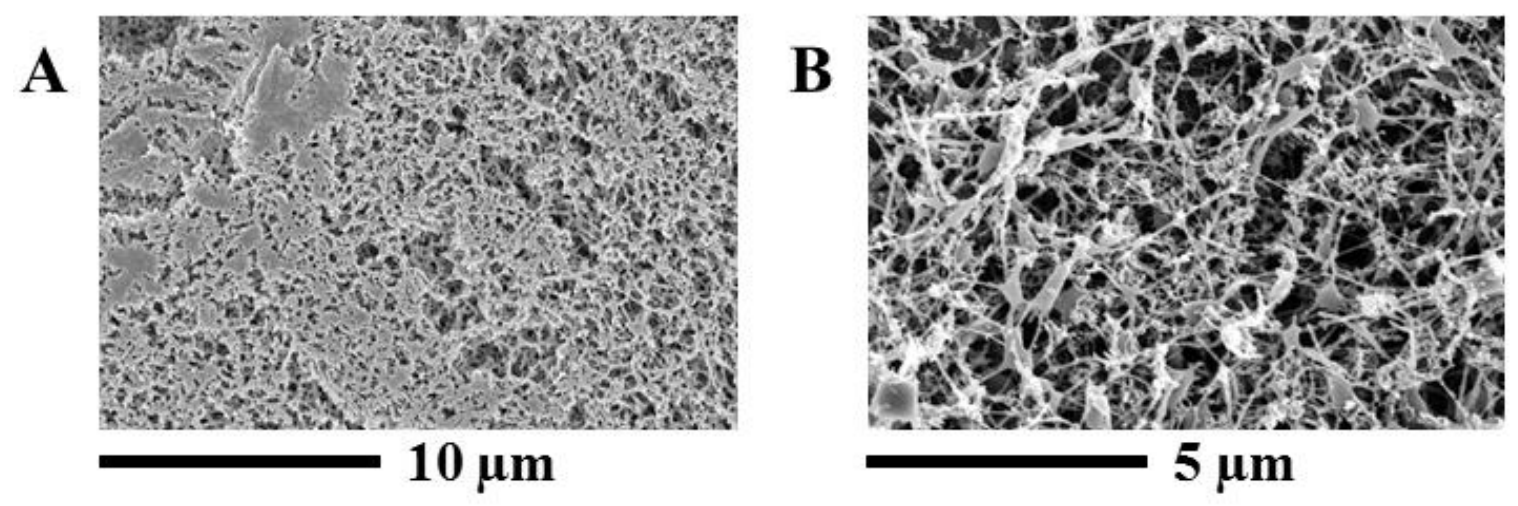

Fig. 38. SEM of clots formed with whole plasma from WT mice. (A) A dissected clot formed with undiluted plasma. (B) A thinner clot specimen obtained with 1:4 diluted plasma.

packed. Clots formed in the presence of FXIIIA better retained their three-dimensional structure throughout the sample preparation process. Analogous to the pure fibrin system, no confident conclusions could be made on differences in fiber thickness for the clots crosslinked by FXIII AP-variants. More importantly, however, an increasing amount of nonfibrous crosslinked protein was observed within the FXIII-containing clots: the least with the W34 (slow activated) and the most with the L34 (fast activated) FXIII AP-variant (Fig. 39). Whether this nonfibrous material represents non-polymerized fibrin or other plasma proteins, it is expected to contribute to overall clot penetrability and mechanical and fibrinolytic stability. 
1

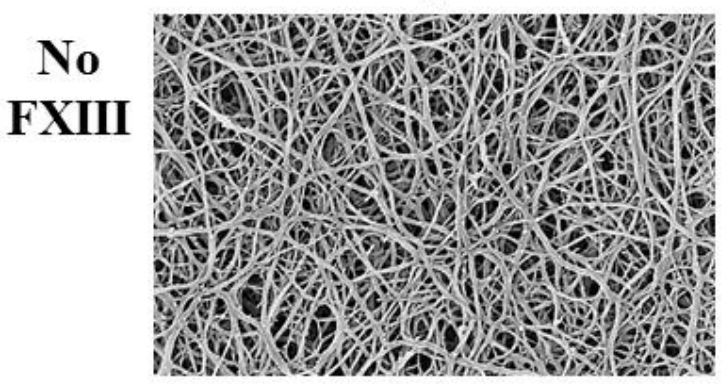

W34

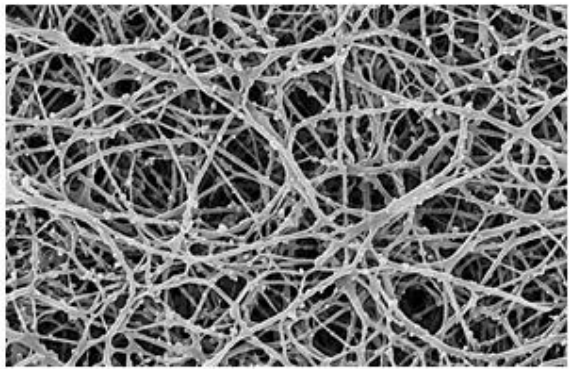

V34

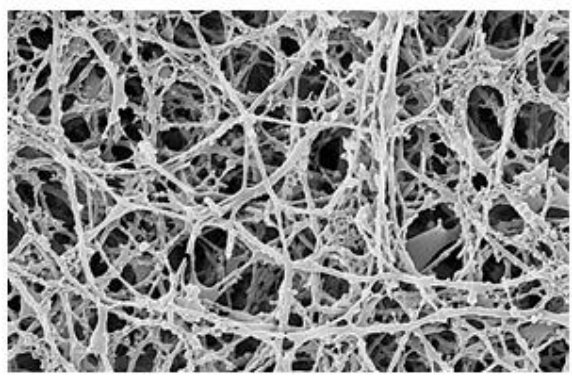

L34

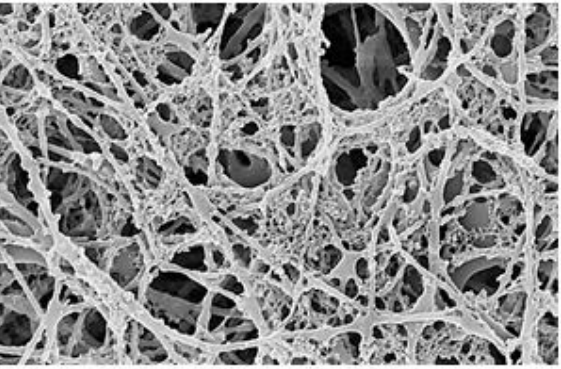

\section{$5 \mu \mathrm{m}$}

Fig. 39. SEM of clots formed with whole plasma (1:4 dilution) from FXIIIA-deficient mice after administration of exogenous FXIIIA AP variants, as well as control clots without FXIIIA. Two sets of clots were studied. For each set, clots were made using the plasma from a single mouse (1 and 2). 


\section{Discussion}

For a better understanding of FXIIIA function in different physiological contexts, one needs to consider the peculiarities of FXIIIA activation environments. It is reasonable to assume that due to highly regulated $\mathrm{Ca}$ homeostasis, nonproteolytic activation pathways leading to FXIIIA $^{\circ \text {,low }}$ and FXIIIA ${ }^{\circ, \text { high }}$ generally operate in a constitutive manner. The activity of FXIIIA as part of ongoing physiological processes within the cells and in some extracellular matrices is thereby expected to be relatively constant. In blood plasma, where concentration of $\mathrm{Ca}^{2+}$ ions is maintained at a low $1.5 \mathrm{mM}$, constitutive generation of FXIIIA $^{\circ \text {,low }}$ species is prevented by association of FXIIIA with the inhibitory FXIIIB (64). In a process that is initiated in exigent cases such as bleeding, the proteolytic removal of activation peptide prompts fast FXIIIA activation. The complexity of this mechanism arises from concomitant activation of FXIIIA with proteolytic conversion of fibrinogen to polymerizing fibrin by the same protease, thrombin (144). In this situation, the rate of FXIIIA* generation and hence the timing of crosslinking activity introduction may have a significant impact on the functional outcome of the clotting process, as observed with the natural L34 FXIIIA polymorphism $(88,89)$.

In previous kinetic studies with synthetic activation peptide sequences $(90-94,96)$,

the Maurer group demonstrated that amino acid residue 34 of the FXIII AP affects the rate of thrombin-catalyzed cleavage of the R37-G38 peptide bond. Altering the activation peptide residue 34 was predicted to change the full length FXIIIA activation rate and thus, to affect the properties of fibrin clot. For experimental validation of the synthetic peptide predictions, a series of full-length FXIIIA V34X variants was generated. The cleavage rates of those variants by WT and mutant I174A thrombin constituted trends similar to those 
observed with synthetic peptides. Furthermore in SDS-PAGE experiments, the extent of fibrin covalent crosslinking correlated with the FXIIIA* generation rate.

Initial attempts of fibrin clot preparation for SEM were conducted following methodology reported by researchers in the blood coagulation field. The coating, likely formed by non-polymerized fibrin(ogen), covered the major surface area of the clots, thus significantly limiting our ability to examine the morphology of the individual fibers. In a recent publication by Macrae et al. (145), such coating, referred to as fibrin biofilm, was proposed to be a product of the fibrin clotting. A major concern associated with that study is the routine use of glutaraldehyde as a fixative agent in clot preparation. As mentioned in Chapter 2, glutaraldehyde is a protein crosslinker. Thus, fixation with glutaraldehyde would covalently stabilize non-polymerized fibrin(ogen) at the outer surface of the clot.

Macrae reported that the film formed as a result of fibrin polymerization at the liquid-air interface. In the current work, such air-liquid interface was also present during fibrin polymerization. At the final stages of preparation for the SEM, fibrin clots were fully suspended in dehydrating solutions. Thus, mounting of multiple samples with the surface, that was air-exposed during clot formation, always facing up is difficult to imagine. Yet, the fibrin film was consistently observed in all clot samples, thus suggesting that such film does not necessarily result from fibrin exposure to the air. Furthermore, from analysis of the clots obtained in the presence of a FXIIIA inhibitor, Macrae and coworkers concluded that film formation did not require FXIIIA-mediated crosslinking of the fibrin molecules. However, no control experiments without use of the crosslinker glutaraldehyde were discussed. In the present work, no film was observed upon elimination of glutaraldehyde from sample preparation, further supporting the artificial nature of the film. The 
morphology of the fibers that were or were not fixed in glutaraldehyde was similar. Therefore, the present study demonstrates that the glutaraldehyde fixation, often necessary to preserve the morphology of cellular structures, does not always benefit preparation of fibrin clots for SEM analysis. In fact, investigation of the FXIIIA-catalyzed crosslinking effect would greatly be hindered by introducing artificial crosslinking into the fibrin clot.

Ariëns and coworkers were the first group to employ a combination of solutionbased techniques and SEM to examine the functional significance of the FXIIIA L34 polymorphism (88). This research group demonstrated that the faster activated FXIIIA L34 introduced more covalent crosslinks than the more prevalent V34 variant, thus resulting in mechanically stronger fibrin clots. The slightly lower turbidity at $340 \mathrm{~nm}$ for clots formed in the presence of L34 was then correlated with thinner fibers observed by SEM. In the current work, additional FXIIIA X34 variants were generated with a broader range of activation rates. The extent of covalent fibrin crosslinking was clearly correlated with the activation rate of FXIIIA AP variants, in a good agreement with observations by Ariëns $e t$ al. Surprisingly, no striking differences were observed between fiber thickness of the clots formed in the presence of those AP variants and the WT thrombin. Such observation was further supported by the same fiber thickness in the FXIII-free clots used as a negative control. Thus, current results suggest that FXIIIA-mediated crosslinking does not have a major impact on fiber thickness.

An appreciable effect of FXIIIA on clot turbidity could only be seen upon significant, non-physiological impairment of fibrin generation by the thrombin mutant I174A. Even with the observed difference in turbidity in solution, SEM analysis of dried samples did not reveal differences in fiber thickness. Therefore, turbidity at $340 \mathrm{~nm}$ likely 
reflected the thickness of undisturbed hydrated fibrin fibers in a stagnant solution. Different turbidity values obtained with the same starting fibrinogen and thrombin concentrations would thus correlate with different packing density of protofibrils within the fiber. Physiologically, this packing density is largely dependent on the efficiency of fibrin generation by thrombin. In addition, fibrin fibers undergo irreversible compaction as they form under constant tensile and pressure stresses in the dynamic plasma environment (146).

Possible experimental limitations of the current work could arise from the fibrinogen preparation used. FXIII-free Peak 1 fibrinogen purified from human plasma does not contain $\gamma^{\prime}$ chains resulting from alternative mRNA splicing. The $\gamma^{\prime}$ fibrinogen constitutes $8-15 \%$ of the total fibrinogen and has been shown to promote branching of the fibers in a polymerizing fibrin clot (147-149). Therefore, the absence of $\gamma^{\prime}$ variant could lead to an altered clot morphology. However, FXIIIA crosslinking is not directed exclusively to the $\gamma^{\prime}$ fibrin, as indicated by the present SDS-page assay, where crosslinking could still be observed in the absence of $\gamma^{\prime}$ (Fig. 34).

Hethershaw and coworkers examined clot structure by SEM using the regular $\gamma / \gamma^{\prime}$ fibrinogen (131). Scrupulous measurements revealed statistically significant differences in fiber thickness for clots formed in the presence $(75 \mathrm{~nm})$ and absence $(86 \mathrm{~nm})$ of FXIIIA. Working with a similar fibrinogen preparation, Ryan et al. (150) reported that inhibition of FXIII did not affect morphology of the fibers. More profound factors affecting fiber thickness were the starting concentrations of fibrinogen and thrombin. Increasing those concentrations led to increased branching, which in turn was correlated with thinner fibers. It is necessary to acknowledge that such conclusive trends were not observed in the present study (Appendices 1-4). This lack of observation could be attributed to the narrower range 
of thrombin and fibrinogen concentrations employed, and to the absence of the branchingpromoting $\gamma^{\prime}$ chain.

Examining the function of FXIIIA AP variants in whole plasma overcame limitations of the pure fibrin system and provided long sought after morphological evidence of the FXIIIA effect on the clot architecture. Besides fibrin fibers, additional protein material was revealed by the SEM analysis. By eliminating the artificial glutaraldehyde crosslinking, it was possible to correlate the amount of this nonfibrous material in the clots with the activation rate of FXIIIA AP variants. The murine plasma employed in the current work contained all the physiological protein factors (except the exogenous FXIIIA). Interestingly, in a proteomic study, Nikolajsen with coworkers (16) identified 48 different FXIIIA substrates present in the crosslinked plasma clot. Thus, the currently observed nonfibrous material could originate from the plasma proteins other than fibrinogen. Regardless of its protein identity, this material significantly contributed to the overall clot structure observed by SEM, and is expected to further increase the mechanical strength of non-diluted plasma clots in vivo.

To summarize, the developed V34X full-length protein model allowed investigation of the effect of timing of FXIIIA activity appearance on the fibrin clot properties. Present work reveals that the rate of proteolytic FXIIIA activation determines the extent of covalent fibrin crosslinking that confers increased mechanical strength of the fibers within a clot. An additional previously overlooked contribution to the clot architecture is provided by FXIIIA-moderated incorporation of nonfibrous protein within the fibrin matrix. 


\section{CHAPTER 7}

\section{RESEARCH SUMMARY AND FUTURE DIRECTIONS}

In recent years, FXIIIA has acquired recognition as a crucial player not only in blood coagulation but also in nonhemostatic events. Despite previous intensive investigations of FXIIIA, critical gaps are still present in understanding biochemical properties of this transglutaminase. FXIIIA is the only member of the transglutaminase family that exists as a homodimer in the zymogen form. In the absence of structural information, the active form has long been thought to be a dimer as well. However, given the proximity of the active sites to the dimer interface, rearrangements would have to occur for the A-subunits to be able to accommodate two large protein substrates and to release the crosslinked product. While some computational work has probed these rearrangements, Chapter 4 of this dissertation describes a series of solution experiments that provide a simple explanation for the ability of FXIIIA to fulfill its function - the dissociation of $\mathrm{A}_{2}$ homodimer as part of activation. Chapter 5 describes investigations that examine FXIIIA oligomeric states further to compare the efficiency of proteolytic and nonproteolytic activation pathways. Nonproteolytic activation is proposed to occur slowly but constitutively, thus constantly supplying active FXIIIA to fulfill nonhemostatic functions. The fast build up of FXIIIA activity during thrombin-mediated proteolytic activation perfectly suits this mechanism to the physiological environment of blood coagulation. A special interest in this activation pathway arises from the concomitant physiological 
activation of fibrinogen and FXIIIA by thrombin. The V34X model described in Chapter 6 permits efficient manipulation of thrombin's ability to activate FXIIIA without affecting the conversion of fibrinogen to fibrin. I now summarize my findings and present perspectives for future factor XIII research.

\section{Probing FXIIIA conformational rearrangements}

A series of solution-based techniques was employed to study changes in the FXIIIA during its activation. Both proteolytic and nonproteolytic activation resulted in a decrease of FXIIIA intrinsic tryptophan fluorescence. The fluorescence decrease from that of the zymogen indicated a change in polarity of the environment surrounding the tryptophan residues, consistent with their increased solvent exposure. Interestingly in the nPAGE experiments, activated FXIIIA forms could not be well resolved. Such a change in electrophoretic mobilities further confirmed global conformational rearrangements that FXIIIA undergoes during activation. In addition, the observations suggested the critical importance of $\mathrm{Ca}^{2+}$ for maintaining activated FXIIIA conformation. Size exclusion chromatography (SEC) revealed different Stokes radii for the zymogen and nonproteolytically activated forms of FXIIIA. In principle, the SEC elution profile of activated FXIIIA was consistent with the reduced molecular weight of this form; however given the asymmetry of FXIIIA globular structure, correlation with the molecular weight is not possible. Furthermore, SEC could not be successfully utilized for studying thrombinactivated FXIIIA due to heavy precipitation of this species.

Sedimentation velocity AUC experiments provided resolution of both proteolytically and nonproteolytically activated FXIIIA. Compared to the dimeric 
zymogen FXIIIA ( $s=7.9 \mathrm{~S}$ ), the values for activated FXIIIA were lower (4.6-4.8 S), indicative of the monomeric state. Notably, the active FXIIIA remained monomeric upon binding of substrate analogs including the small molecule iodoacetamide and the peptide K9-DON.

Given the noncovalent stabilization of the dimer, zymogen FXIIIA 2 has been viewed as a self-interacting system in equilibrium. Sedimentation equilibrium AUC provided an estimated $K_{\mathrm{D}}$ of $8 \mathrm{nM}$ for the zymogen FXIIIA 2 . Sedimentation equilibrium data for the activated FXIIIA forms were also fitted to the monomer-dimer equilibrium model, yielding a $K_{\mathrm{D}}$ of $90 \mathrm{mM}$ for nonproteolytically activated and $220 \mathrm{mM}$ for proteolytically activated FXIIIA. Such high mM concentrations required for the interaction of activated A-subunits are certainly impossible to occur in a physiological setting. Therefore, sedimentation equilibrium AUC results provided critical support that active FXIIIA exists as a monomer at physiological concentrations.

A few naturally occurring and experimental amino acid substitutions (R11Q, R260C, R260L, and G562R) disrupt the dimer interactions in zymogen FXIIIA2. Following site-directed mutagenesis of the FXIIIA gene, these mutants were expressed as GST-fusion proteins in E. coli. The mutant FXIIIA proteins were only soluble while still attached to the dimeric GST-tag. Proteolytic cleavage of GST caused immediate precipitation of FXIIIA, indicating solution instability of the mutant proteins and stressing the necessity of the dimeric state for zymogen FXIIIA 2 . In contrast to amino acid mutations at the dimer interface, substitution at a more distant site within the catalytic core domain tyrosine 283 (Y283C) caused a significant conformational distortion in the FXIIIA molecule but had a milder effect on the ability of zymogen to dimerize. 
Experiments presented in this dissertation were performed exclusively on FXIIIA without its plasma partner FXIIIB. Since dissociation of the B-subunits from the Asubunits after cleavage of the activation peptide has already been well documented, the physiological presence of FXIIIB is not expected to affect post-cleavage rearrangements of FXIIIA in plasma. The presence of FXIIIB in the experimental samples would, however, affect hydrodynamic modeling of the sedimentation data.

Of more interest is the oligomeric state of FXIIIB itself. Plasma FXIII has been routinely described as a heterocomplex of $\mathrm{A}_{2}$ and $\mathrm{B}_{2}$ dimers. Studies by Chung et al. (73) and Ichinose et al. (151) suggested the dimeric state for free FXIIIB. These conclusions were based on gel filtration results, and caution should be taken in correlating protein peak elution volumes with the molecular weights. This concern is especially important for FXIIIB, which is even less symmetric than FXIIIA. Moreover, noncovalent dimeric interaction is concentration-dependent and, while FXIIIB subunits may dimerize at the relatively high concentrations required for detection by size exclusion chromatography, the oligomeric state at lower concentrations found in plasma is unknown. Carrel and coworkers (152) performed electron microscopy and density gradient centrifugation and suggested a monomeric state for FXIIIB. The Carrell group conclusions were questioned by Bishop et al. (105) on the basis of incorrect interpretation of the molecular dimensions. Thus, the dimeric state of FXIIIB has not been conclusively established. The deciphering of the interactions between the B-subunits and the A-B hetero-associations warrants future research. 


\section{Dynamic and functional implications of FXIIIA activation pathways}

Sedimentation velocity AUC along with catalytic activity assays were employed to compare FXIIIA forms resulting from different activation pathways. Full proteolytic activation of FXIIIA could be achieved with physiological thrombin amounts within minutes. Furthermore, analysis of partially thrombin-activated FXIIIA (approximately half of the A-subunits cleaved) indicated that for most of the dimeric zymogen species cleavage of a single activation peptide was sufficient to promote dissociation to monomers. Both cleaved and noncleaved FXIIIA species possessed equal enzymatic activity. Thus, proteolysis by thrombin presents the fastest mechanism of FXIIIA activation. However, the resulting FXIIIA* species tend to aggregate, suggesting a relatively short lifetime of this FXIIIA form.

The rate of nonproteolytic FXIIIA activation was dependent on the $\mathrm{Ca}^{2+}$ concentration. Incubation of FXIIIA with $100 \mathrm{mM} \mathrm{Ca}^{2+}\left(\mathrm{FXIIIA}^{\circ, \text { high }}\right)$ resulted in full dissociation of the A-subunits within $30 \mathrm{~min}$, a rate comparable to proteolytic activation. At $25 \mathrm{mM} \mathrm{Ca}^{2+}$ - as encountered in the bone ECM, only $20 \%$ dissociation of FXIIIA was observed after 30 min. Unlike, thrombin-activated FXIIIA*, no precipitation of the FXIIIA $^{\text {,high }}$ form was observed. At $4 \mathrm{mM} \mathrm{Ca}^{2+}$, approximately $20 \%$ dissociation of FXIIIA was only observed after $6 \mathrm{~h}$. Accurate quantitation of monomeric protein became difficult at longer incubation times due to progressive precipitation of the FXIIIA ${ }^{\text {,low }}$.

Thus, it was possible to isolate uniformly monomeric FXIIIA* and FXIIIA ${ }^{\circ \text {,high }}$ forms for further investigation. Comparison of sedimentation properties of these forms revealed greater conformational heterogeneity for FXIIIA*. The higher solubility of FXIIIA ${ }^{\circ \text {,high }}$ species was correlated with a greater amount of bound $\mathrm{Ca}^{2+}$. Kinetic 
comparisons of the two FXIIIA forms revealed that FXIIIA* interacts with the glutamine substrate more readily (indicated by lower apparent $K_{\mathrm{m}}$ ) than does FXIIIA ${ }^{\circ \text {,high }}$. The latter, however, provided slightly faster transglutaminase reaction turnover (higher apparent $\left.V_{\max }\right)$. Therefore, the activity results corroborated proposed conformational and dynamic differences between the two FXIIIA forms. Interestingly, previous reports suggested occupancy of two $\mathrm{Ca}^{2+}$ sites in activated FXIIIA molecule. A recently published crystal structure of FXIIIA ${ }^{\circ, \text { high }}$ with covalently bound inhibitor reveals three $\mathrm{Ca}^{2+}$ binding sites. With that in mind, the current AUC and activity observations led to a proposal that in the presence of low $\mathrm{mM} \mathrm{Ca}^{2+}$, such as in plasma, saturation of the third $\mathrm{Ca}^{2+}$ site in FXIIIA is coordinated with binding of the glutamine substrate. By contrast, in environments where $\mathrm{Ca}^{2+}$ concentration is relatively high, such as bone ECM, the $3^{\text {rd }} \mathrm{Ca}^{2+}$ site may be saturated even in the free enzyme. This saturation results in lower FXIIIA ${ }^{\circ \text {,high }}$ affinity to the glutamine substrate but increases the solution stability of this form.

The possibilities raised by observations of the current project call for a more detailed kinetic evaluation of proteolytically and non-proteolytically activated FXIIIA. Future research could focus on probing FXIIIA specificity not only for the glutamine but also for the amine substrates. In the present work, the direct chromogenic assay provided the best kinetic information; however, the poor solubility of DMPDA along with its absorbance properties limited the range of concentrations that could be employed in the assay. Under certain conditions, the kinetic response of FXIIIA to increasing glutamine substrate concentrations was reminiscent of that for enzymes with negative cooperativity. Since the current work demonstrated a monomeric state of active FXIIIA, this kinetic response may suggest hysteresis in FXIIIA catalytic behavior. The limitations of the 
DMPDA assay, however, would not allow such conclusions to be made.

Another activity assay employed is based on detection of the ammonia released during the transglutaminase reaction. Since this assay does not rely on the chromogenic amine FXIIIA substrate, different amine donors can be utilized, with a greater concentration range than is feasible for DMPDA. In the present work, a downside of the ammonia release assay manifested in the vulnerability of the coupling enzyme glutamate dehydrogenase to $100 \mathrm{mM} \mathrm{Ca}^{2+}$ required for the $\mathrm{FXIII}^{\circ, \text { high }}$ form. Additional experiments indicate that once activated by $100 \mathrm{mM} \mathrm{Ca}^{2+}$ (a quick way to obtain uniformly monomeric species), the FXIIIA ${ }^{\text {ohigh }}$ form remains monomeric and demonstrates the same level of activity even at $25 \mathrm{mM} \mathrm{Ca}^{2+}$. Above all, the coupled ammonia release assay may be used in future experiments investigating catalytic properties of both FXIIIA* and FXIIIA ${ }^{\circ \text {,high }}$.

The MDC activity assay qualitatively confirmed the results of the DMPDA assay. In addition, MDC assay allowed study of FXIIIA catalytic action on the physiological protein substrate fibrinogen $\alpha \mathrm{C}$ at physiological concentrations. However, the MDC assay in the form employed for current research is demanding in terms of time and labor. Better throughput may be achieved in future work by direct measurement of the fluorescence of the reacted glutamine substrate. Instead of electrophoresis, the separation from excess nonreacted MDC may be achieved by precipitating the protein with trichloroacetic acid followed by centrifugation. After resuspension of the resulting pellet, the amount of incorporated MDC can be measured using fluorimetry.

Another aspect that needs more elucidation is binding of $\mathrm{Ca}^{2+}$ by FXIIIA. Available reports indicate $K_{\mathrm{D}}$ values of $100 \mu \mathrm{M}$ for the first, zymogenic $\mathrm{Ca}^{2+}$ site. The affinities of the remaining two putative $\mathrm{Ca}^{2+}$ sites in the FXIIIA molecule are unknown. A conventional 
method, such as ITC, may provide reliable information on the stoichiometry of FXIIIA-Ca interactions and affinities of $\mathrm{Ca}^{2+}$ binding sites. However, a titration approach will only reveal the $\mathrm{Ca}^{2+}$ amounts that can bind to FXIIIA. For determination of $\mathrm{Ca}^{2+}$ that actually does bind to FXIIIA under different conditions, the FXIIIA forms would have to be isolated followed by quantification of bound $\mathrm{Ca}^{2+}$ per weight of the protein. Such isolation of FXIIIA without loss of the bound $\mathrm{Ca}^{2+}$ seems challenging, if not impossible. More importantly, and demanding further investigation is the possibility that the binding of $\mathrm{Ca}^{2+}$ by FXIIIA-glutamine substrate complex favors transamidation over deamidation. A series of kinetic experiments is expected to estimate the effect of $\mathrm{Ca}^{2+}$ concentration on FXIIIA*catalyzed transamidation versus deamidation of the glutamine substrate. A lower $\mathrm{Ca}^{2+}$ requirement for deamidation than transamidation would be consistent with that idea.

\section{V34X model of FXIIIA proteolytic activation}

FXIIIA variants with V34X amino acid substitutions were recombinantly expressed in E. coli. The thrombin-catalyzed cleavage of the V34X variants was monitored by SDSPAGE and densitometric analysis. FXIIIA variants were ranked in terms of their cleavage rates: L34>>V34>F34>>W34. In a series of fibrin crosslinking assays, FXIIIA variants were activated by thrombin simultaneously with fibrinogen. Thrombin cleavage rates were correlated with the extent of covalent crosslinking between fibrin $\alpha$ and $\gamma$ chains introduced by V34X variants. A relatively small difference between V34 and F34 cleavage rates did not lead to distinct differences in fibrin crosslinking by these two variants. Thus, the most crosslinks were introduced by the fastest activated L34, followed by V34 $\approx \mathrm{F} 34$, and the least amount of fibrin crosslinking was observed in the presence of slow activated W34 
FXIIIA.

FXIIIA variants activated faster or slower by thrombin would begin crosslinking at different stages of fibrin polymerization. The results of fibrin turbidity assays indicated that the presence of FXIIIA as a competing thrombin substrate slightly delayed and decelerated the fibrin polymerization. However, a clear dependence of this effect on FXIIIA activation rate could only be observed upon nonphysiological impairment of fibrin formation by a mutant I174A thrombin.

SEM analysis of fibrin clots prepared with purified fibrinogen revealed no major effect of FXIIIA on the fibrin fiber thickness or morphology. Both thick and thin fibers were observed in the clots regardless of the amount of covalent fibrin crosslinks. Closer examination revealed that the thicker fibers resulted from fusion of thinner fibers. More importantly, the glutaraldehyde fixation, routinely used in preparation of clot samples for SEM, was shown to result in artificial fibrin coating on the clot surface. Elimination of glutaraldehyde from the sample preparation simplified subsequent imaging of the specimens without affecting the fibrin network morphology. Still, the extent of fibrin crosslinking by different FXIIIA variants did not correlate with fiber thickness, although mechanical strength differences were qualitatively observed. Thus far, the technically simple SDS-PAGE assay has been the most useful approach for monitoring crosslinking of fibrin chains and predicting mechanical strength of the fibrin clot.

FXIIIA variants were then introduced into whole plasma from FXIIIA-deficient mice. SEM analysis of the resulting clots revealed previously overlooked morphological evidence for incorporation of nonfibrous protein material into the clot. This protein material may significantly contribute to the mechanical properties of the clot in vivo. 
Importantly, the extent of the nonfibrous protein incorporation correlated with the FXIIIA activation rate.

Thus, the tunable FXIII AP sequences allowed ex vivo manipulation of fibrin clot properties. Besides presenting an efficient experimental model for studying fibrin clotting, the V34X FXIIIA variants may be implemented in future development of fibrin-based biomaterials with desired mechanical properties and even tissue engineering. Another interesting perspective is matching the designed FXIII V34X with thrombin variants. Thrombin mutants with reduced procoagulant activity have been developed to hinder the extent of fibrin clotting $(153,154)$. Current interest is in testing the ability of those anticoagulant thrombins to activate FXIIIA V34X variants thus still allowing non-fibrin crosslinking reactions to occur. As an example in the present work, I174A thrombin cleaved fibrinogen significantly slower than the WT thrombin, but efficiently activated W34 FXIIIA. Such a mutant thrombin-FXIIIA pair may provide an additional benefit of normal FXIIIA crosslinking activity in environments with reduced fibrin coagulation. Further screening of thrombin-FXIIIA combinations is suggested as an immediate continuation of the current research project.

\section{Final opinion:}

\section{On the prospective pharmaceutical targeting of FXIIIA}

The presented research was aimed to explore uncharacterized fundamental properties of FXIIIA, and through such exploration, expand the current understanding of FXIIIA physiological function. A retrospective evaluation of the entire investigation leads to a recurrent notion that it is the activation pathway diversity that makes FXIIIA stand out among the transglutaminase family of enzymes. This activation diversity may be a key to 
differential therapeutic targeting of FXIIIA function in a variety of pathophysiological events, a concept yet to be recognized and addressed in pharmaceutical development.

The thrombin activated FXIIIA* form currently presents the most attractive pharmacological target. However, not only has the FXIIIA* been elusive from crystallographic efforts, but also a challenging subject of solution experiments. Nevertheless, once the conformational difference between FXIIIA* and FXIII ${ }^{\circ}$,high is recognized, it is possible to overcome the lack of detailed structural information and begin searching for pharmaceutical agents that specifically target either form. The crystal structures of non-proteolytically activated $\mathrm{FXIII}^{\circ}$,high in complexes with inhibitors are available from the Stieler group (PDB 4KTY, 5MHM, $5 \mathrm{MHN}$, 5MHO). It is then feasible to screen active site targeting compounds that will confer specificity to FXIIIA ${ }^{\circ \text {,high }}$ and eliminate those affecting the FXIIIA*. Should this orthosteric design fail, a search for allosteric effectors may be more successful. Previous studies in the Maurer group involving chemical modification and hydrogen-deuterium exchange suggested better solvent accessibility of the $\beta$-sandwich region of FXIIIA*, while $\beta$-barrel 1 was more solventexposed in FXIIIA ${ }^{\circ \text {,high }}$. These local structural differences may serve as a starting point for allosteric targeting of FXIIIA.

Therapeutic specificity towards the coagulation-active FXIIIA* may be achieved by controlling its proteolytic activation. In this work, the variation in FXIIIA* generation rate has been demonstrated to affect fibrin crosslinking. Faster proteolytic activation (and hence, greater amount of FXIIIA*) during blood coagulation results in a larger extent of fibrin crosslinking and incorporation of other proteins into the clot. In this direction, allosteric agents specifically limiting thrombin's ability to activate FXIIIA and not 
fibrinogen, may be promising. The V34X activation model provides an interesting basis for development of anticoagulants. The tunable $\mathbf{X V P R} \downarrow G$ amino acid sequence may serve as a prodrug linker whose cleavage by active thrombin will release the pharmaceutical agent, such as recombinant plasmin, at a desirable rate and specifically at the clotting site. This approach would also ensure that neither FXIIIA ${ }^{\circ \text {,low }}$ nor FXIIIA ${ }^{\circ \text { high }}$ are affected. 


\section{REFERENCES}

1. Muszbek, L., Yee, V. C., and Hevessy, Z. (1999) Blood coagulation factor XIII: structure and function. Thromb Res 94, 271-305

2. Schroeder, V., and Kohler, H. P. (2016) Factor XIII: Structure and Function. Semin Thromb Hemost 42, 422-428

3. Komaromi, I., Bagoly, Z., and Muszbek, L. (2011) Factor XIII: novel structural and functional aspects. J Thromb Haemost 9, 9-20

4. Muszbek, L., Bereczky, Z., Bagoly, Z., Komaromi, I., and Katona, E. (2011) Factor XIII: a coagulation factor with multiple plasmatic and cellular functions. Physiol $\operatorname{Rev}$ 91, 931-972

5. Adany, R., and Bardos, H. (2003) Factor XIII subunit A as an intracellular transglutaminase. Cell Mol Life Sci 60, 1049-1060

6. Adany, R. (1996) Intracellular factor XIII: cellular distribution of factor XIII subunit a in humans. Semin Thromb Hemost 22, 399-408

7. Adany, R., Kiss, A., and Muszbek, L. (1987) Factor XIII: a marker of mono- and megakaryocytopoiesis. Br J Haematol 67, 167-172

8. Adany, R., Belkin, A., Vasilevskaya, T., and Muszbek, L. (1985) Identification of blood coagulation factor XIII in human peritoneal macrophages. Eur J Cell Biol 38, $171-173$

9. Adany, R., Bardos, H., Antal, M., Modis, L., Sarvary, A., Szucs, S., and Balogh, I. (2001) Factor XIII of blood coagulation as a nuclear crosslinking enzyme. Thromb Haemost 85, 845-851

10. Smith, S. A., Travers, R. J., and Morrissey, J. H. (2015) How it all starts: Initiation of the clotting cascade. Crit Rev Biochem Mol Biol 50, 326-336

11. Weisel, J. W. (2005) Fibrinogen and fibrin. Adv Protein Chem 70, 247-299 
12. Weisel, J. W., and Litvinov, R. I. (2013) Mechanisms of fibrin polymerization and clinical implications. Blood 121, 1712-1719

13. Takagi, T., and Doolittle, R. F. (1974) Amino acid sequence studies on factor XIII and the peptide released during its activation by thrombin. Biochemistry 13, 750756

14. Hornyak, T. J., Bishop, P. D., and Shafer, J. A. (1989) Alpha-thrombin-catalyzed activation of human platelet factor XIII: relationship between proteolysis and factor XIIIa activity. Biochemistry 28, 7326-7332

15. Bagoly, Z., Koncz, Z., Harsfalvi, J., and Muszbek, L. (2012) Factor XIII, clot structure, thrombosis. Thromb Res 129, 382-387

16. Nikolajsen, C. L., Dyrlund, T. F., Poulsen, E. T., Enghild, J. J., and Scavenius, C. (2014) Coagulation factor XIIIa substrates in human plasma: identification and incorporation into the clot. J Biol Chem 289, 6526-6534

17. Wolberg, A. S. (2018) Fibrinogen and factor XIII: newly recognized roles in venous thrombus formation and composition. Curr Opin Hematol 25, 358-364

18. Whyte, C. S., Mitchell, J. L., and Mutch, N. J. (2017) Platelet-Mediated Modulation of Fibrinolysis. Semin Thromb Hemost 43, 115-128

19. Swieringa, F., Spronk, H. M. H., Heemskerk, J. W. M., and van der Meijden, P. E. J. (2018) Integrating platelet and coagulation activation in fibrin clot formation. Res Pract Thromb Haemost 2, 450-460

20. Iismaa, S. E., Mearns, B. M., Lorand, L., and Graham, R. M. (2009) Transglutaminases and disease: lessons from genetically engineered mouse models and inherited disorders. Physiol Rev 89, 991-1023

21. Dickneite, G., Herwald, H., Korte, W., Allanore, Y., Denton, C. P., and Matucci Cerinic, M. (2015) Coagulation factor XIII: a multifunctional transglutaminase with clinical potential in a range of conditions. Thromb Haemost 113, 686-697

22. Castellino, F. J., and Ploplis, V. A. (2005) Structure and function of the plasminogen/plasmin system. Thromb Haemost 93, 647-654

23. Lijnen, H. R., and Collen, D. (1995) Mechanisms of physiological fibrinolysis. Baillieres Clin Haematol 8, 277-290 
24. Muszbek, L., Bagoly, Z., Bereczky, Z., and Katona, E. (2008) The involvement of blood coagulation factor XIII in fibrinolysis and thrombosis. Cardiovasc Hematol Agents Med Chem 6, 190-205

25. Byrnes, J. R., and Wolberg, A. S. (2016) Newly-Recognized Roles of Factor XIII in Thrombosis. Semin Thromb Hemost 42, 445-454

26. Walton, B. L., Byrnes, J. R., and Wolberg, A. S. (2015) Fibrinogen, red blood cells, and factor XIII in venous thrombosis. J Thromb Haemost 13 Suppl 1, S208-215

27. Bereczky, Z., and Muszbek, L. (2011) Factor XIII and venous thromboembolism. Semin Thromb Hemost 37, 305-314

28. Zeerleder, S., Schroeder, V., Lammle, B., Wuillemin, W. A., Hack, C. E., and Kohler, H. P. (2007) Factor XIII in severe sepsis and septic shock. Thromb Res 119, 311-318

29. Loof, T. G., Morgelin, M., Johansson, L., Oehmcke, S., Olin, A. I., Dickneite, G., Norrby-Teglund, A., Theopold, U., and Herwald, H. (2011) Coagulation, an ancestral serine protease cascade, exerts a novel function in early immune defense. Blood 118, 2589-2598

30. Frick, I. M., Bjorck, L., and Herwald, H. (2007) The dual role of the contact system in bacterial infectious disease. Thromb Haemost 98, 497-502

31. Wang, Z., Wilhelmsson, C., Hyrsl, P., Loof, T. G., Dobes, P., Klupp, M., Loseva, O., Morgelin, M., Ikle, J., Cripps, R. M., Herwald, H., and Theopold, U. (2010) Pathogen entrapment by transglutaminase--a conserved early innate immune mechanism. PLoS Pathog 6, e1000763

32. Deicke, C., Chakrakodi, B., Pils, M. C., Dickneite, G., Johansson, L., Medina, E., and Loof, T. G. (2016) Local activation of coagulation factor XIII reduces systemic complications and improves the survival of mice after Streptococcus pyogenes M1 skin infection. Int J Med Microbiol 306, 572-579

33. Friedrich, R., Panizzi, P., Fuentes-Prior, P., Richter, K., Verhamme, I., Anderson, P. J., Kawabata, S., Huber, R., Bode, W., and Bock, P. E. (2003) Staphylocoagulase is a prototype for the mechanism of cofactor-induced zymogen activation. Nature 425, 535-539

34. Hendrix, H., Lindhout, T., Mertens, K., Engels, W., and Hemker, H. C. (1983) Activation of human prothrombin by stoichiometric levels of staphylocoagulase. $J$ Biol Chem 258, 3637-3644 
35. Thomer, L., Schneewind, O., and Missiakas, D. (2013) Multiple ligands of von Willebrand factor-binding protein (vWbp) promote Staphylococcus aureus clot formation in human plasma. J Biol Chem 288, 28283-28292

36. Levi, M. (2018) Pathogenesis and diagnosis of disseminated intravascular coagulation. Int J Lab Hematol 40 Suppl 1, 15-20

37. Kim, D. Y., Cho, S. H., Takabayashi, T., and Schleimer, R. P. (2015) Chronic Rhinosinusitis and the Coagulation System. Allergy Asthma Immunol Res 7, 421430

38. Levi, M., and van der Poll, T. (2010) Inflammation and coagulation. Crit Care Med 38, S26-34

39. Wygrecka, M., Jablonska, E., Guenther, A., Preissner, K. T., and Markart, P. (2008) Current view on alveolar coagulation and fibrinolysis in acute inflammatory and chronic interstitial lung diseases. Thromb Haemost 99, 494-501

40. Johnson, K. A., Rose, D. M., and Terkeltaub, R. A. (2008) Factor XIIIA mobilizes transglutaminase 2 to induce chondrocyte hypertrophic differentiation. J Cell Sci 121, 2256-2264

41. Raghu, H., Cruz, C., Rewerts, C. L., Frederick, M. D., Thornton, S., Mullins, E. S., Schoenecker, J. G., Degen, J. L., and Flick, M. J. (2015) Transglutaminase factor XIII promotes arthritis through mechanisms linked to inflammation and bone erosion. Blood 125, 427-437

42. Falanga, A., Panova-Noeva, M., and Russo, L. (2009) Procoagulant mechanisms in tumour cells. Best Pract Res Clin Haematol 22, 49-60

43. Falanga, A., Marchetti, M., and Vignoli, A. (2013) Coagulation and cancer: biological and clinical aspects. J Thromb Haemost 11, 223-233

44. Falanga, A., and Marchetti, M. (2018) Hemostatic biomarkers in cancer progression. Thromb Res 164 Suppl 1, S54-S61

45. Breitwieser, G. E. (2008) Extracellular calcium as an integrator of tissue function. Int J Biochem Cell Biol 40, 1467-1480

46. Nakashima, T., Hayashi, M., and Takayanagi, H. (2012) New insights into osteoclastogenic signaling mechanisms. Trends Endocrinol Metab 23, 582-590 
47. Nakano, Y., Al-Jallad, H. F., Mousa, A., and Kaartinen, M. T. (2007) Expression and localization of plasma transglutaminase factor XIIIA in bone. J Histochem Cytochem 55, 675-685

48. Hoac, B., Nelea, V., Jiang, W., Kaartinen, M. T., and McKee, M. D. (2017) Mineralization-inhibiting effects of transglutaminase-crosslinked polymeric osteopontin. Bone 101, 37-48

49. Mousa, A., Cui, C., Song, A., Myneni, V. D., Sun, H., Li, J. J., Murshed, M., Melino, G., and Kaartinen, M. T. (2017) Transglutaminases factor XIII-A and TG2 regulate resorption, adipogenesis and plasma fibronectin homeostasis in bone and bone marrow. Cell Death Differ 24, 844-854

50. Tarantino, U., Oliva, F., Taurisano, G., Orlandi, A., Pietroni, V., Candi, E., Melino, G., and Maffulli, N. (2009) FXIIIA and TGF-beta over-expression produces normal musculo-skeletal phenotype in TG2-/- mice. Amino Acids 36, 679-684

51. Al-Jallad, H. F., Nakano, Y., Chen, J. L., McMillan, E., Lefebvre, C., and Kaartinen, M. T. (2006) Transglutaminase activity regulates osteoblast differentiation and matrix mineralization in MC3T3-E1 osteoblast cultures. Matrix Biol 25, 135-148

52. Nurminskaya, M., and Kaartinen, M. T. (2006) Transglutaminases in mineralized tissues. Front Biosci 11, 1591-1606

53. Cordell, P. A., Newell, L. M., Standeven, K. F., Adamson, P. J., Simpson, K. R., Smith, K. A., Jackson, C. L., Grant, P. J., and Pease, R. J. (2015) Normal Bone Deposition Occurs in Mice Deficient in Factor XIII-A and Transglutaminase 2. Matrix Biol 43, 85-96

54. Sun, H., and Kaartinen, M. T. (2018) Transglutaminase activity regulates differentiation, migration and fusion of osteoclasts via affecting actin dynamics. $J$ Cell Physiol 233, 7497-7513

55. Cui, C., Wang, S., Myneni, V. D., Hitomi, K., and Kaartinen, M. T. (2014) Transglutaminase activity arising from Factor XIIIA is required for stabilization and conversion of plasma fibronectin into matrix in osteoblast cultures. Bone $\mathbf{5 9}$, $127-138$

56. Al-Jallad, H. F., Myneni, V. D., Piercy-Kotb, S. A., Chabot, N., Mulani, A., Keillor, J. W., and Kaartinen, M. T. (2011) Plasma membrane factor XIIIA transglutaminase activity regulates osteoblast matrix secretion and deposition by affecting microtubule dynamics. PLoS One 6, e15893 
57. Piercy-Kotb, S. A., Mousa, A., Al-Jallad, H. F., Myneni, V. D., Chicatun, F., Nazhat, S. N., and Kaartinen, M. T. (2012) Factor XIIIA transglutaminase expression and secretion by osteoblasts is regulated by extracellular matrix collagen and the MAP kinase signaling pathway. J Cell Physiol 227, 2936-2946

58. Laki, K., and Lorand, L. (1948) On the Solubility of Fibrin Clots. Science 108, 280

59. Keillor, J. W., Clouthier, C. M., Apperley, K. Y., Akbar, A., and Mulani, A. (2014) Acyl transfer mechanisms of tissue transglutaminase. Bioorg Chem 57, 186-197

60. Folk, J. E. (1983) Mechanism and basis for specificity of transglutaminasecatalyzed epsilon-(gamma-glutamyl) lysine bond formation. Adv Enzymol Relat Areas Mol Biol 54, 1-56

61. Lorand, L., and Konishi, K. (1964) Activation of the fibrin stabilizing factor of plasma by thrombin. Arch Biochem Biophys 105, 58-67

62. Muszbek, L., Haramura, G., and Polgar, J. (1995) Transformation of cellular factor XIII into an active zymogen transglutaminase in thrombin-stimulated platelets. Thromb Haemost 73, 702-705

63. Muszbek, L., Polgar, J., and Boda, Z. (1993) Platelet Factor XIII becomes active without the release of activation peptide during platelet activation. Thromb Haemostasis 69, 282-285

64. Polgar, J., Hidasi, V., and Muszbek, L. (1990) Non-proteolytic activation of cellular protransglutaminase (placenta macrophage factor XIII). Biochem J 267, 557-560

65. Curtis, C. G., Brown, K. L., Credo, R. B., Domanik, R. A., Gray, A., Stenberg, P., and Lorand, L. (1974) Calcium-dependent unmasking of active center cysteine during activation of fibrin stabilizing factor. Biochemistry 13, 3774-3780

66. Muszbek, L., Ariens, R. A., Ichinose, A., and Isth Ssc Subcommittee On Factor, X. (2007) Factor XIII: recommended terms and abbreviations. J Thromb Haemost 5, 181-183

67. Holbrook, J. J., Cooke, R. D., and Kingston, I. B. (1973) The amino acid sequence around the reactive cysteine residue in human plasma Factor XII. Biochem J 135, 901-903

68. Ichinose, A., Hendrickson, L. E., Fujikawa, K., and Davie, E. W. (1986) Amino acid sequence of the a subunit of human factor XIII. Biochemistry 25, 6900-6906 
69. Yee, V. C., Pedersen, L. C., Le Trong, I., Bishop, P. D., Stenkamp, R. E., and Teller, D. C. (1994) Three-dimensional structure of a transglutaminase: human blood coagulation factor XIII. Proc Natl Acad Sci U S A 91, 7296-7300

70. Yee, V. C., Le Trong, I., Bishop, P. D., Pedersen, L. C., Stenkamp, R. E., and Teller, D. C. (1996) Structure and function studies of factor XIIIa by x-ray crystallography. Semin Thromb Hemost 22, 377-384

71. Weiss, M. S., Metzner, H. J., and Hilgenfeld, R. (1998) Two non-proline cis peptide bonds may be important for factor XIII function. FEBS Lett 423, 291-296

72. Stieler, M., Weber, J., Hils, M., Kolb, P., Heine, A., Buchold, C., Pasternack, R., and Klebe, G. (2013) Structure of active coagulation factor XIII triggered by calcium binding: basis for the design of next-generation anticoagulants. Angew Chem Int Ed Engl 52, 11930-11934

73. Chung, S. I., Lewis, M. S., and Folk, J. E. (1974) Relationships of the catalytic properties of human plasma and platelet transglutaminases (activated blood coagulation factor XIII) to their subunit structures. J Biol Chem 249, 940-950

74. Vysokovsky, A., Rosenberg, N., Dardik, R., Seligsohn, U., and Inbal, A. (2006) Effect of four missense mutations in the factor XIII A-subunit gene on protein stability: studies with recombinant proteins. Blood Coagul Fibrinolysis 17, 125130

75. Ichinose, A., Tsukamoto, H., Izumi, T., Yamazaki, T., Togashi, M., Takamatsu, J., Saito, H., and Umeyama, H. (1998) Arg260-Cys mutation in severe factor XIII deficiency: conformational change of the A subunit is predicted by molecular modelling and mechanics. Br J Haematol 101, 264-272

76. Maeda, S., Zhang, W. G., Souri, M., Yee, V. C., and Ichinose, A. (2012) Impaired dimer assembly and decreased stability of naturally recurring R260C mutant A subunit for coagulation factor XIII. J Biochem 152, 471-478

77. Zhang, W. G., Souri, M., and Ichinose, A. (2013) Proteosomal degradation of naturally recurring R260C missense and exon-IV deletion mutants of factor XIII A-subunit expressed in mammalian cells. Haemophilia 19, 415-419

78. Souri, M., and Ichinose, A. (2001) Impaired protein folding, dimer formation, and heterotetramer assembly cause intra- and extracellular instability of a Y283C mutant of the A subunit for coagulation factor XIII. Biochemistry 40, 13413-13420 
79. Handrkova, H., Schroeder, V., and Kohler, H. P. (2015) The activation peptide of coagulation factor XIII is vital for its expression and stability. J Thromb Haemost 13, 1449-1458

80. Janus, T. J., Lewis, S. D., Lorand, L., and Shafer, J. A. (1983) Promotion of thrombin-catalyzed activation of factor XIII by fibrinogen. Biochemistry 22, 62696272

81. Shafer, J. A., Lewis, S. D., Janus, T. J., and Lorand, L. (1986) Effect of fibrinogen and $\mathrm{Ca} 2+$ on the thrombin-catalyzed proteolytic event that triggers activation of factor XIII. Ann N Y Acad Sci 485, 134-139

82. Hornyak, T. J., and Shafer, J. A. (1991) Role of calcium ion in the generation of factor XIII activity. Biochemistry 30, 6175-6182

83. Credo, R. B., Curtis, C. G., and Lorand, L. (1978) Ca2+-related regulatory function of fibrinogen. Proc Natl Acad Sci U S A 75, 4234-4237

84. Turner, B. T., Sabo, T. M., Wilding, D., and Maurer, M. C. (2004) Mapping of factor XIII solvent accessibility as a function of activation state using chemical modification methods. Biochemistry 43, 9755-9765

85. Sabo, T. M., Brasher, P. B., and Maurer, M. C. (2007) Perturbations in factor XIII resulting from activation and inhibition examined by solution based methods and detected by MALDI-TOF MS. Biochemistry 46, 10089-10101

86. Woofter, R. T., and Maurer, M. C. (2011) Role of calcium in the conformational dynamics of factor XIII activation examined by hydrogen-deuterium exchange coupled with MALDI-TOF MS. Arch Biochem Biophys 512, 87-95

87. Gupta, S., Biswas, A., Akhter, M. S., Krettler, C., Reinhart, C., Dodt, J., Reuter, A., Philippou, H., Ivaskevicius, V., and Oldenburg, J. (2016) Revisiting the mechanism of coagulation factor XIII activation and regulation from a structure/functional perspective. Sci Rep 6, 30105

88. Ariens, R. A., Philippou, H., Nagaswami, C., Weisel, J. W., Lane, D. A., and Grant, P. J. (2000) The factor XIII V34L polymorphism accelerates thrombin activation of factor XIII and affects cross-linked fibrin structure. Blood 96, 988-995

89. Duval, C., Ali, M., Chaudhry, W. W., Ridger, V. C., Ariens, R. A., and Philippou, H. (2016) Factor XIII A-Subunit V34L Variant Affects Thrombus Cross-Linking in a Murine Model of Thrombosis. Arterioscler Thromb Vasc Biol 36, 308-316 
90. Trumbo, T. A., and Maurer, M. C. (2000) Examining thrombin hydrolysis of the factor XIII activation peptide segment leads to a proposal for explaining the cardioprotective effects observed with the factor XIII V34L mutation. J Biol Chem 275, 20627-20631

91. Trumbo, T. A., and Maurer, M. C. (2003) V34I and V34A substitutions within the factor XIII activation peptide segment (28-41) affect interactions with the thrombin active site. Thromb Haemost 89, 647-653

92. Isetti, G., and Maurer, M. C. (2004) Thrombin activity is unaltered by N-terminal truncation of factor XIII activation peptides. Biochemistry 43, 4150-4159

93. Isetti, G., and Maurer, M. C. (2004) Probing thrombin's ability to accommodate a V34F substitution within the factor XIII activation peptide segment (28-41). J Pept Res 63, 241-252

94. Isetti, G., and Maurer, M. C. (2007) Employing mutants to study thrombin residues responsible for factor XIII activation peptide recognition: a kinetic study. Biochemistry 46, 2444-2452

95. Jadhav, M. A., Isetti, G., Trumbo, T. A., and Maurer, M. C. (2010) Effects of introducing fibrinogen Aalpha character into the factor XIII activation peptide segment. Biochemistry 49, 2918-2924

96. Jadhav, M. A., Lucas, R. C., Goldsberry, W. N., and Maurer, M. C. (2011) Design of Factor XIII V34X activation peptides to control ability to interact with thrombin mutants. Biochim Biophys Acta 1814, 1955-1963

97. Van Holde, K. E. (1971) Physical biochemistry, Prentice-Hall, Englewood Cliffs, N.J.

98. Lebowitz, J., Lewis, M. S., and Schuck, P. (2002) Modern analytical ultracentrifugation in protein science: a tutorial review. Protein Sci 11, 2067-2079

99. Dam, J., and Schuck, P. (2004) Calculating sedimentation coefficient distributions by direct modeling of sedimentation velocity concentration profiles. Methods Enzymol 384, 185-212

100. Lyman, C. E., Newbury, D. E., Goldstein, J. I., Williams, D. B., Roming Jr, A. D., Armstrong, J. T., Echlin, P., Fiori, C. E., Joy, D. C., Lifshin, E., and Peters, K. (1990) Scanning electron microscopy, $x$-ray microanalysis, and analytical electron microscopy a laboratory workbook, New york, Plenum Press 
101. Murtey, M. D., and Ramasamy, P. (2016) Sample Preparations for Scanning Electron Microscopy - Life Sciences.

102. Katona, E., Haramura, G., Karpati, L., Fachet, J., and Muszbek, L. (2000) A simple, quick one-step ELISA assay for the determination of complex plasma factor XIII (A2B2). Thromb Haemost 83, 268-273

103. Katona, E. E., Ajzner, E., Toth, K., Karpati, L., and Muszbek, L. (2001) Enzymelinked immunosorbent assay for the determination of blood coagulation factor XIII A-subunit in plasma and in cell lysates. J Immunol Methods 258, 127-135

104. Demirin, H., Ozhan, H., Ucgun, T., Celer, A., Bulur, S., Cil, H., Gunes, C., and Yildirim, H. A. (2011) Normal range of mean platelet volume in healthy subjects: Insight from a large epidemiologic study. Thromb Res 128, 358-360

105. Bishop, P. D., Teller, D. C., Smith, R. A., Lasser, G. W., Gilbert, T., and Seale, R. L. (1990) Expression, purification, and characterization of human factor XIII in Saccharomyces cerevisiae. Biochemistry 29, 1861-1869

106. Bishop, P. D., Lasser, G. W., Le Trong, I., Stenkamp, R. E., and Teller, D. C. (1990) Human recombinant factor XIII from Saccharomyces cerevisiae. Crystallization and preliminary X-ray data. J Biol Chem 265, 13888-13889

107. Lai, T. S., Santiago, M. A., Achyuthan, K. E., and Greenberg, C. S. (1994) Purification and characterization of recombinant human coagulant factor XIII Achains expressed in E. coli. Protein Expr Purif 5, 125-132

108. Hettasch, J. M., and Greenberg, C. S. (1994) Analysis of the catalytic activity of human factor XIIIa by site-directed mutagenesis. J Biol Chem 269, 28309-28313

109. Lai, T. S., Achyuthan, K. E., Santiago, M. A., and Greenberg, G. S. (1994) Carboxyl-terminal truncation of recombinant factor XIII A-chains. Characterization of minimum structural requirement for transglutaminase activity. J Biol Chem 269, 24596-24601

110. Hettasch, J. M., Peoples, K. A., and Greenberg, C. S. (1997) Analysis of factor XIII substrate specificity using recombinant human factor XIII and tissue transglutaminase chimeras. J Biol Chem 272, 25149-25156

111. Lai, T. S., Slaughter, T. F., Peoples, K. A., and Greenberg, C. S. (1999) Sitedirected mutagenesis of the calcium-binding site of blood coagulation factor XIIIa. J Biol Chem 274, 24953-24958 
112. Smith, K. A., Adamson, P. J., Pease, R. J., Brown, J. M., Balmforth, A. J., Cordell, P. A., Ariens, R. A., Philippou, H., and Grant, P. J. (2011) Interactions between factor XIII and the alphaC region of fibrinogen. Blood 117, 3460-3468

113. Studier, F. W. (2014) Stable expression clones and auto-induction for protein production in E. coli. Methods Mol Biol 1091, 17-32

114. Karpati, L., Penke, B., Katona, E., Balogh, I., Vamosi, G., and Muszbek, L. (2000) A modified, optimized kinetic photometric assay for the determination of blood coagulation factor XIII activity in plasma. Clin Chem 46, 1946-1955

115. Fickenscher, K., Aab, A., and Stuber, W. (1991) A photometric assay for blood coagulation factor XIII. Thromb Haemost 65, 535-540

116. Studier, F. W. (2005) Protein production by auto-induction in high density shaking cultures. Protein Expr Purif 41, 207-234

117. Jabs, A., Weiss, M. S., and Hilgenfeld, R. (1999) Non-proline cis peptide bonds in proteins. J Mol Biol 286, 291-304

118. Schmidpeter, P. A., Koch, J. R., and Schmid, F. X. (2015) Control of protein function by prolyl isomerization. Biochim Biophys Acta 1850, 1973-1982

119. Pathak, B. K., Banerjee, S., Mondal, S., Chakraborty, B., Sengupta, J., and Barat, C. (2017) Unfolded protein exhibits antiassociation activity toward the 50S subunit facilitating 70S ribosome dissociation. FEBS J 284, 3915-3930

120. Katona, E., Penzes, K., Csapo, A., Fazakas, F., Udvardy, M. L., Bagoly, Z., Orosz, Z. Z., and Muszbek, L. (2014) Interaction of factor XIII subunits. Blood 123, 17571763

121. Mary, A., Achyuthan, K. E., and Greenberg, C. S. (1988) The binding of divalent metal ions to platelet factor XIII modulates its proteolysis by trypsin and thrombin. Arch Biochem Biophys 261, 112-121

122. Yee, V. C., Pedersen, L. C., Bishop, P. D., Stenkamp, R. E., and Teller, D. C. (1995) Structural evidence that the activation peptide is not released upon thrombin cleavage of factor XIII. Thromb Res 78, 389-397

123. Ackers, G. K. (1970) Analytical gel chromatography of proteins. Adv Protein Chem 24, 343-446 
124. Cooke, R. D., and Holbrook, J. J. (1974) The calcium-induced dissociation of human plasma clotting factor XIII. Biochem J 141, 79-84

125. Traut, T. W. (1994) Dissociation of enzyme oligomers: a mechanism for allosteric regulation. Crit Rev Biochem Mol Biol 29, 125-163

126. Hashimoto, K., Nishi, H., Bryant, S., and Panchenko, A. R. (2011) Caught in selfinteraction: evolutionary and functional mechanisms of protein homooligomerization. Phys Biol 8, 035007

127. Pinkas, D. M., Strop, P., Brunger, A. T., and Khosla, C. (2007) Transglutaminase 2 undergoes a large conformational change upon activation. PLoS Biol 5, e327

128. Clouthier, C. M., Mironov, G. G., Okhonin, V., Berezovski, M. V., and Keillor, J. W. (2012) Real-time monitoring of protein conformational dynamics in solution using kinetic capillary electrophoresis. Angew Chem Int Ed Engl 51, 12464-12468

129. Kristiansen, G. K., and Andersen, M. D. (2011) Reversible activation of cellular factor XIII by calcium. J Biol Chem 286, 9833-9839

130. Eckert, R. L., Kaartinen, M. T., Nurminskaya, M., Belkin, A. M., Colak, G., Johnson, G. V., and Mehta, K. (2014) Transglutaminase regulation of cell function. Physiol Rev 94, 383-417

131. Hethershaw, E. L., Cilia La Corte, A. L., Duval, C., Ali, M., Grant, P. J., Ariens, R. A., and Philippou, H. (2014) The effect of blood coagulation factor XIII on fibrin clot structure and fibrinolysis. J Thromb Haemost 12, 197-205

132. Breuksch, I., Weinert, M., and Brenner, W. (2016) The role of extracellular calcium in bone metastasis. J Bone Oncol 5, 143-145

133. de Macedo, P., Marrano, C., and Keillor, J. W. (2000) A direct continuous spectrophotometric assay for transglutaminase activity. Anal Biochem 285, 16-20

134. Maravall, M., Mainen, Z. F., Sabatini, B. L., and Svoboda, K. (2000) Estimating intracellular calcium concentrations and buffering without wavelength ratioing. Biophys J 78, 2655-2667

135. Frieden, C. (1970) Kinetic aspects of regulation of metabolic processes. The hysteretic enzyme concept. J Biol Chem 245, 5788-5799 
136. Mouapi, K. N., Bell, J. D., Smith, K. A., Ariens, R. A., Philippou, H., and Maurer, M. C. (2016) Ranking reactive glutamines in the fibrinogen alphaC region that are targeted by blood coagulant factor XIII. Blood 127, 2241-2248

137. Heil, A., Weber, J., Buchold, C., Pasternack, R., and Hils, M. (2013) Differences in the inhibition of coagulation factor XIII-A from animal species revealed by Michael Acceptor- and thioimidazol based blockers. Thromb Res 131, e214-222

138. Al-Horani, R. A., Karuturi, R., Lee, M., Afosah, D. K., and Desai, U. R. (2016) Allosteric Inhibition of Factor XIIIa. Non-Saccharide Glycosaminoglycan Mimetics, but Not Glycosaminoglycans, Exhibit Promising Inhibition Profile. PLoS One 11, e0160189

139. Case, A., and Stein, R. L. (2003) Kinetic analysis of the action of tissue transglutaminase on peptide and protein substrates. Biochemistry 42, 9466-9481

140. Lewis, B. A., Freyssinet, J. M., and Holbrook, J. J. (1978) An equilibrium study of metal ion binding to human plasma coagulation factor XIII. Biochem J 169, 397402

141. Folk, J. E., Cole, P. W., and Mullooly, J. P. (1968) Mechanim of action of guinea pig liver transglutaminase. V. The hydrolysis reaction. J Biol Chem 243, 418-427

142. Balogh, I., Szoke, G., Karpati, L., Wartiovaara, U., Katona, E., Komaromi, I., Haramura, G., Pfliegler, G., Mikkola, H., and Muszbek, L. (2000) Val34Leu polymorphism of plasma factor XIII: biochemistry and epidemiology in familial thrombophilia. Blood 96, 2479-2486

143. Wartiovaara, U., Mikkola, H., Szoke, G., Haramura, G., Karpati, L., Balogh, I., Lassila, R., Muszbek, L., and Palotie, A. (2000) Effect of Val34Leu polymorphism on the activation of the coagulation factor XIII-A. Thrombosis and haemostasis $\mathbf{8 4}$, 595-600

144. Brummel, K. E., Butenas, S., and Mann, K. G. (1999) An integrated study of fibrinogen during blood coagulation. J Biol Chem 274, 22862-22870

145. Macrae, F. L., Duval, C., Papareddy, P., Baker, S. R., Yuldasheva, N., Kearney, K. J., McPherson, H. R., Asquith, N., Konings, J., Casini, A., Degen, J. L., Connell, S. D., Philippou, H., Wolberg, A. S., Herwald, H., and Ariens, R. A. (2018) A fibrin biofilm covers blood clots and protects from microbial invasion. $J$ Clin Invest 128, 3356-3368 
146. Liang, X., Chernysh, I., Purohit, P. K., and Weisel, J. W. (2017) Phase transitions during compression and decompression of clots from platelet-poor plasma, plateletrich plasma and whole blood. Acta Biomater 60, 275-290

147. Cooper, A. V., Standeven, K. F., and Ariens, R. A. (2003) Fibrinogen gamma-chain splice variant gamma' alters fibrin formation and structure. Blood 102, 535-540

148. Collet, J. P., Nagaswami, C., Farrell, D. H., Montalescot, G., and Weisel, J. W. (2004) Influence of gamma' fibrinogen splice variant on fibrin physical properties and fibrinolysis rate. Arterioscler Thromb Vasc Biol 24, 382-386

149. Allan, P., Uitte de Willige, S., Abou-Saleh, R. H., Connell, S. D., and Ariens, R. A. (2012) Evidence that fibrinogen gamma' directly interferes with protofibril growth: implications for fibrin structure and clot stiffness. J Thromb Haemost 10, 10721080

150. Ryan, E. A., Mockros, L. F., Weisel, J. W., and Lorand, L. (1999) Structural origins of fibrin clot rheology. Biophys J 77, 2813-2826

151. Souri, M., Kaetsu, H., and Ichinose, A. (2008) Sushi domains in the B subunit of factor XIII responsible for oligomer assembly. Biochemistry 47, 8656-8664

152. Carrell, N. A., Erickson, H. P., and McDonagh, J. (1989) Electron microscopy and hydrodynamic properties of factor XIII subunits. J Biol Chem 264, 551-556

153. Arosio, D., Ayala, Y. M., and Di Cera, E. (2000) Mutation of W215 compromises thrombin cleavage of fibrinogen, but not of PAR-1 or protein C. Biochemistry 39, 8095-8101

154. Pineda, A. O., Chen, Z. W., Caccia, S., Cantwell, A. M., Savvides, S. N., Waksman, G., Mathews, F. S., and Di Cera, E. (2004) The anticoagulant thrombin mutant W215A/E217A has a collapsed primary specificity pocket. J Biol Chem 279, 39824-39828 


\section{APPENDIX 1}

Sample SEM images of clots formed with human FXIII-free fibrinogen

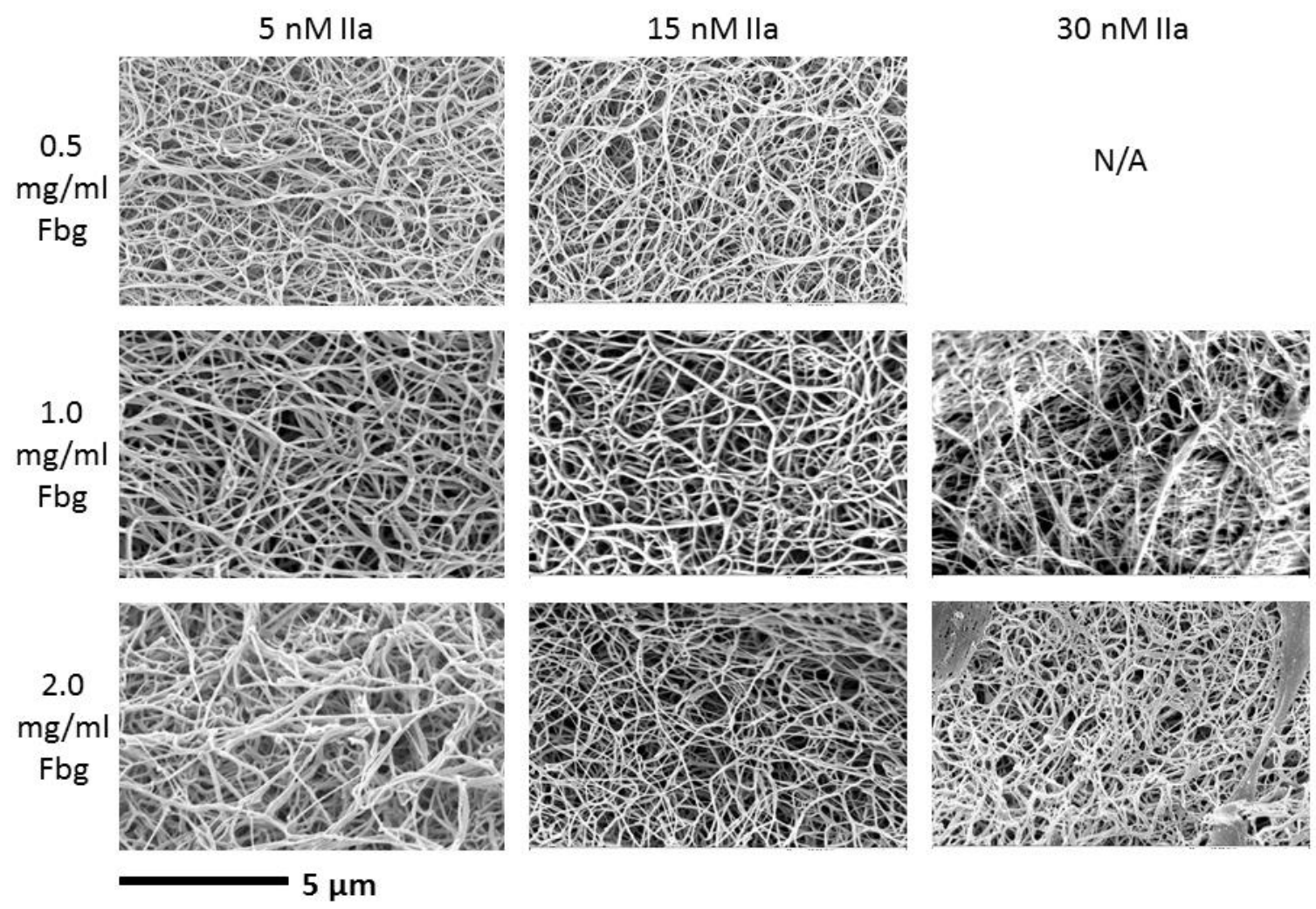




\section{APPENDIX 2}

Sample SEM images of clots formed with human fibrinogen and W34 FXIIIA
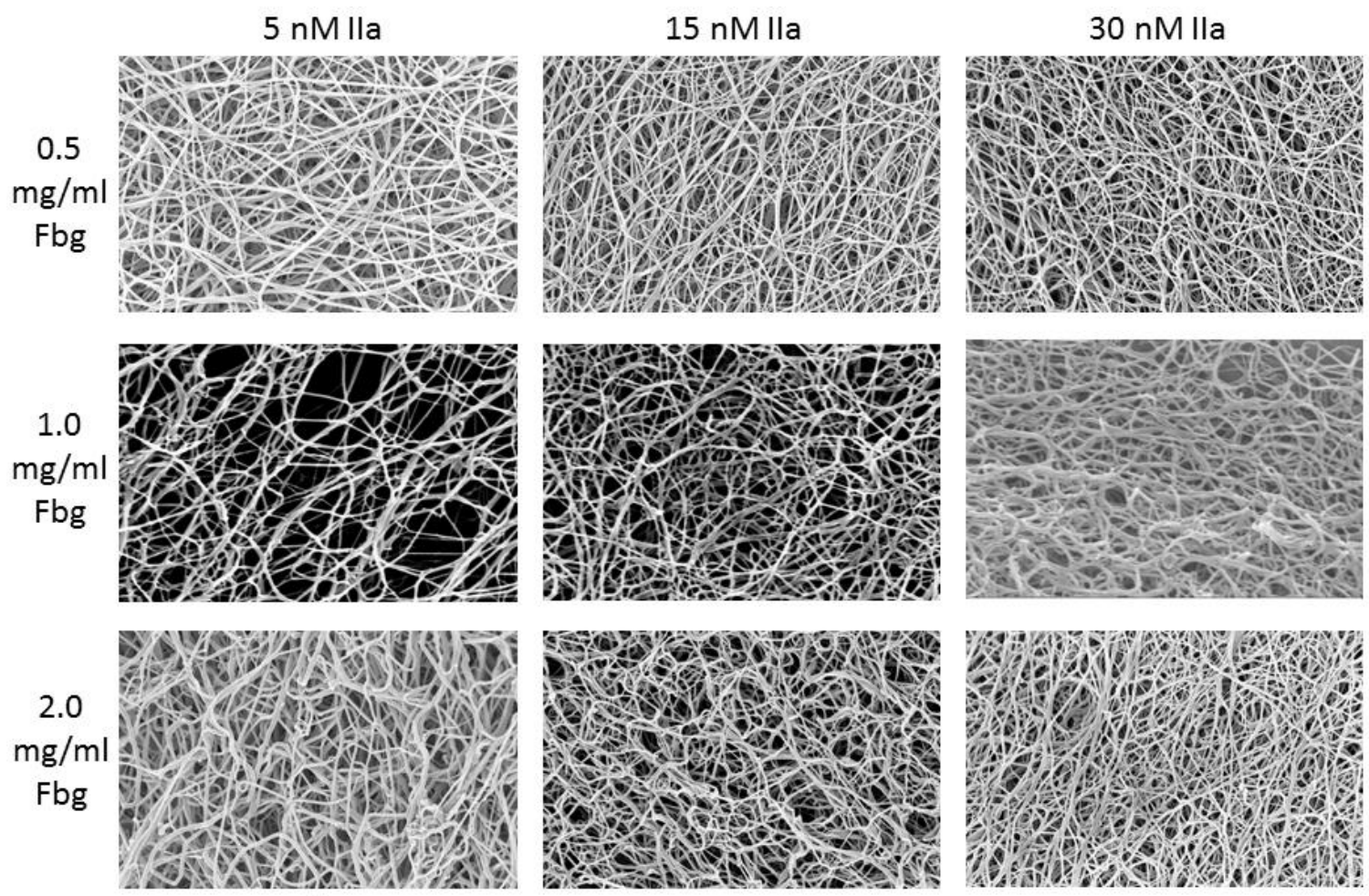

$5 \mu \mathrm{m}$ 
APPENDIX 3

Sample SEM images of clots formed with human fibrinogen and V34 FXIIIA
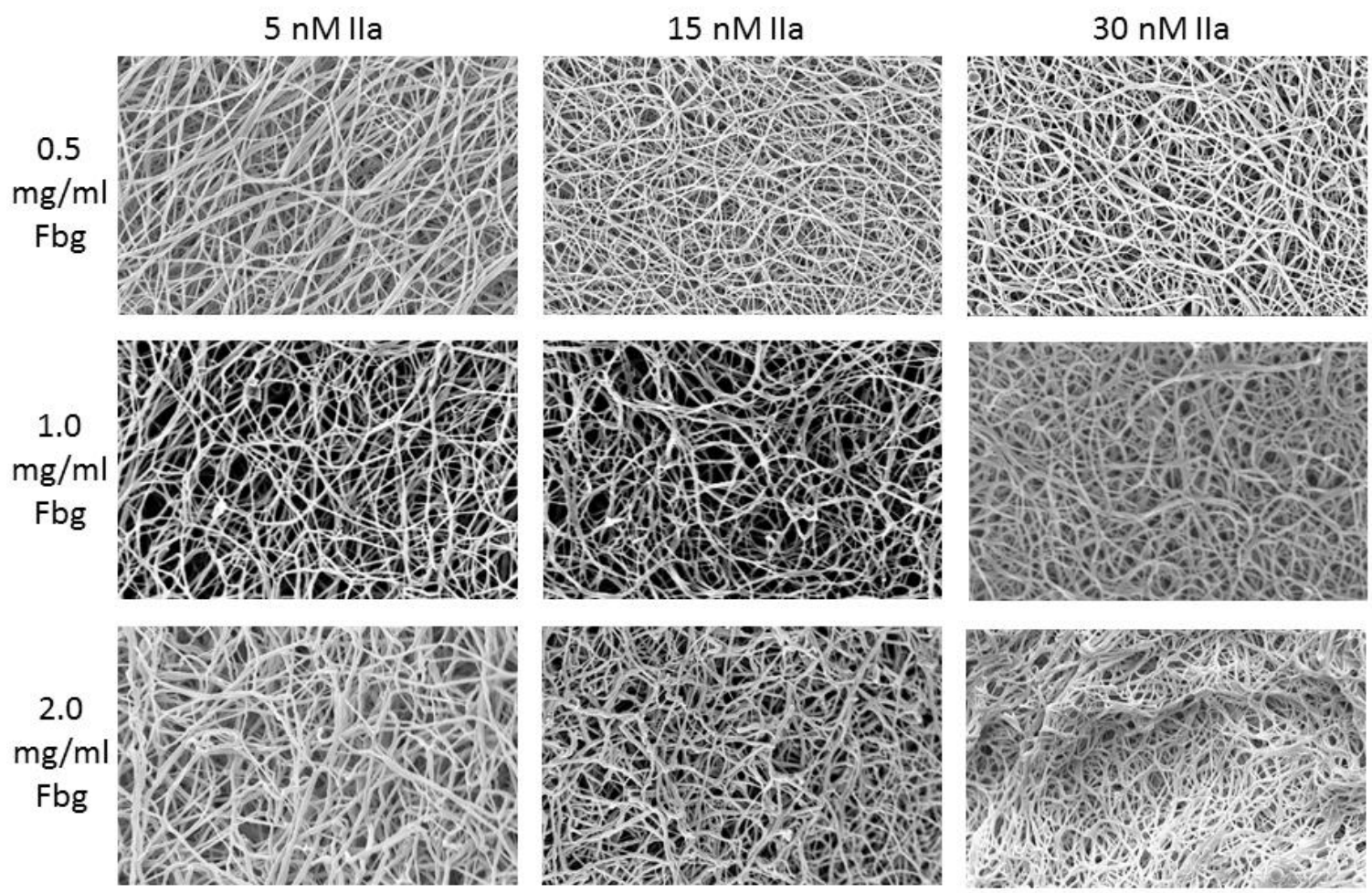

$5 \mu \mathrm{m}$ 
APPENDIX 4

Sample SEM images of clots formed with human fibrinogen and L34 FXIIIA

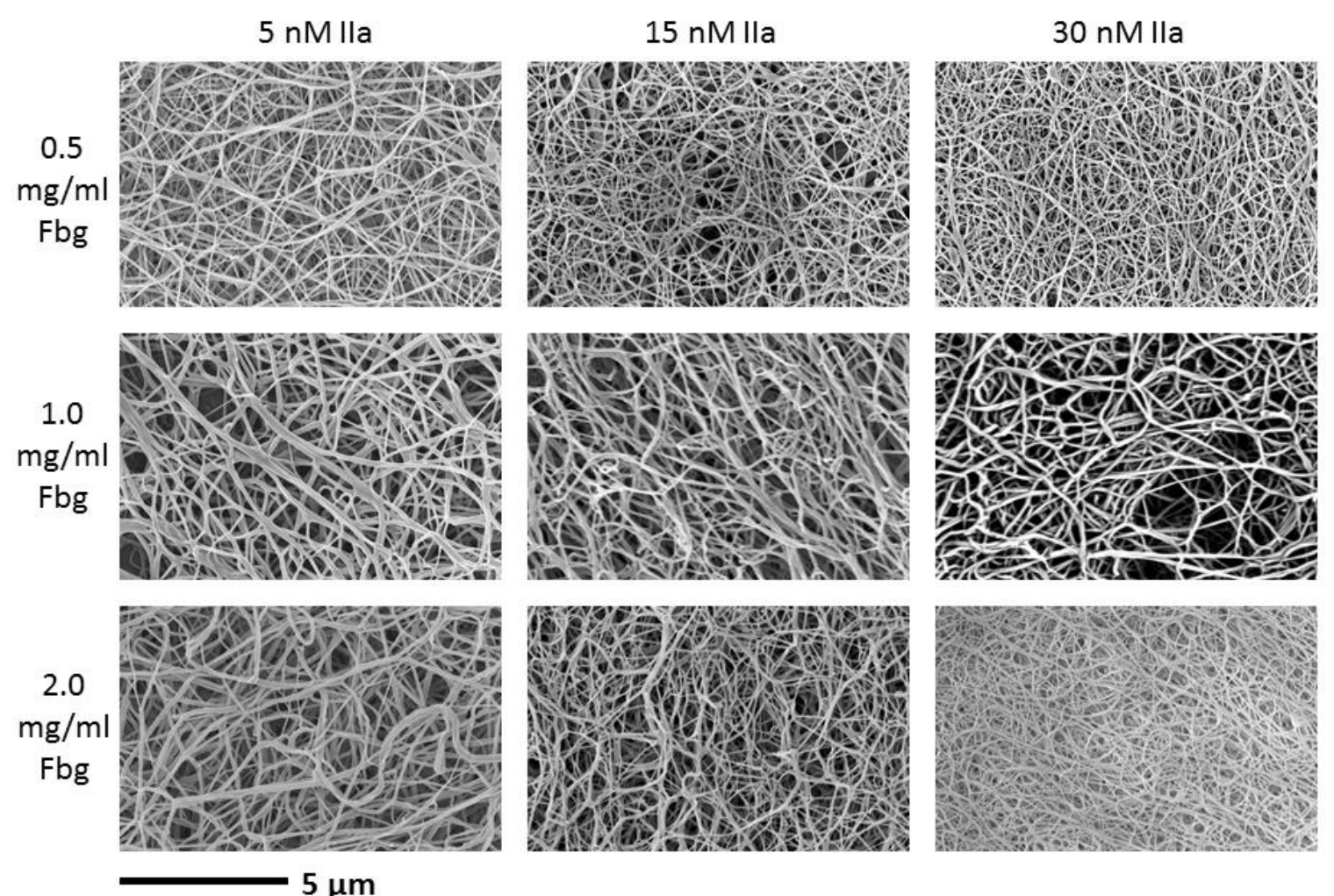




\section{APPENDIX 5}

\section{Abbreviations}

\begin{tabular}{|c|c|}
\hline FXIII & Coagulation factor XIII \\
\hline $\mathrm{RBC}$ & Red blood cells \\
\hline $\mathrm{TF}$ & Tissue factor \\
\hline ECM & Extracellular matrix \\
\hline DIC & Disseminated intravascular coagulation \\
\hline COL 1 & Type 1 collagen \\
\hline $\mathrm{pFN}$ & Plasma fibronectin \\
\hline TG2 & Transglutaminase 2 \\
\hline AUC & Analytical ultracentrifugation \\
\hline SV & Sedimentation velocity \\
\hline $\mathrm{SE}$ & Sedimentation equilibrium \\
\hline SEM & Scanning electron microscopy \\
\hline HMDS & hexamethyldisilazane \\
\hline WT & Wild type \\
\hline PSP & PreScission protease \\
\hline GST & Glutathione S-transferase \\
\hline TBS & Tris buffered saline \\
\hline AP & Activation peptide \\
\hline PPACK & $D$-phenylalanyl-prolyl-arginyl chloromethyl ketone \\
\hline DMSO & Dimethyl sulfoxide \\
\hline DON & 6-diazo-5-oxo-norleucine \\
\hline IAA & Iodoacetamide \\
\hline PBS & Phosphate buffered saline \\
\hline SEC & Size exclusion chromatography \\
\hline nPAGE & Non-denaturing polyacrylamide gel electrophoresis \\
\hline FXIIIA* & FXIIIA activated by thrombin in the presence of $4 \mathrm{mM} \mathrm{Ca}^{2+}$ \\
\hline FXIIIA $^{\circ, \text { low }}$ & FXIIIA activated nonproteolytically by low $\mathrm{mM} \mathrm{Ca}{ }^{2+}$ \\
\hline FXIIIA $^{\circ, \text { high }}$ & FXIIIA activated nonproteolytically by high $(>25 \mathrm{mM}) \mathrm{Ca}^{2+}$ \\
\hline MDC & Monodansylcadaverine \\
\hline DMPDA & $N, N$-dimethyl-1,4-phenylenediamine \\
\hline GEE & Glycine ethyl ester \\
\hline GDH & Glutamate dehydrogenase \\
\hline HMW & High molecular weight \\
\hline FESEM & Field emission scanning electron microscope \\
\hline
\end{tabular}




\title{
CURRICULUM VITAE
}

\author{
Boris Anokhin
}

Department of Chemistry, University of Louisville, Louisville KY 40208

Email: boris.anokhin@louisville.edu

\section{EDUCATION University of Louisville, Louisville, KY, USA}

Ph.D. Chemistry (concentration in Biochemistry), December 2018

Perm State University, Perm, Russia

Graduate program Microbiology, 2010-2013

B.S. Biology (concentration in Microbiology), 2003-2008

\section{RESEARCH EXPERIENCE}

Department of Chemistry, University of Louisville, Louisville, KY

Graduate Assistant 2013-2018; supported by NIH grant 2015-2018

Research Mentor - Dr. Muriel C. Maurer

Oligomeric state of blood coagulation Factor XIIIA (plasma transglutaminase)

- Employed gel electrophoresis, size exclusion chromatography, analytical ultracentrifugation, and site-directed mutagenesis along with recombinant protein expression in E. coli.

- Demonstrated for the first time that homodimeric zymogen Factor XIIIA dissociates into monomers upon activation and transitions to an active transglutaminase. Proposed stabilizing role of dimeric state of the zymogen in a physiological setting.

Proteolytic and nonproteolytic activation of Factor XIIIA

- Employed a series of enzymatic evaluations (spectrophotometric, end-point SDS-PAGE assays).

- Demonstrated that proteolytic activation of Factor XIIIA by thrombin is more efficient than nonproteolytic activation by $\mathrm{Ca}^{2+}$ ions.

- Revealed that nonproteolytically activated FXIIIA is less accessible for its substrates than proteolytically activated enzyme.

- In a physiological context, proteolytical activation results in fast build up of FXIIIA fibrin-stabilizing activity. Nonproteolytically activated FXIIIA exhibits low constitutive activity in non-coagulation environments. Observed differences between the two active forms may serve as a basis for compartment-specific pharmaceutical management of FXIIIA function. 
Influence of Factor XIII on fibrin clot architecture

- Generated FXIIIA activation peptide substitutions (Val 34 Xaa) with altered activation rates.

- Employed electrophoretic assays to demonstrate different extents of fibrin crosslinking by these Factor XIIIA variants.

- Introduced FXIIIA Val 34 Xaa variants into murine FXIIIA-deficient plasma. Studied morphology of the resulting clots using scanning electron microscopy. Demonstrated that FXIIIA activation kinetics directly impacts the structure and stability of a fibrin clot.

Perm State University, Perm, Russia

Undergraduate and graduate research, 2003-2013

Research programs in microbiology:

Mechanisms of Alkanotrophic Rhodococcus Adaptation to Hostile Environments;

Phthalic Acid Utilization by Soil Heterotrophic Bacteria.

- Employed conventional microbiological methods, HPLC, gas chromatography, PCR, and DNA sequencing.

- Characterized adaptive role of morphogenic developmental cycle of Rhodococcus actinobacteria.

- Isolated and characterized bacterial strains capable of biodegradation of xenobiotic compounds.

\section{PUBLICATIONS}

1. Anokhin B.A., Dean W.L., Maurer M.C. [Manuscript in preparation] Proteolytic and nonproteolytic Factor XIII activation mechanisms result in conformationally and functionally different enzymatic forms.

2. Anokhin B.A., Stribinskis V., Dean W.L., Maurer M.C. (2017) Activation of Factor XIII is accompanied by a change in oligomerization state, FEBS J. 284 (22), 3849-3861.

3. Jadhav M.A., Goldsberry W.N., Zink S.E., Lamb K.N., Simmons K.E., Riposo C.M., Anokhin B.A., Maurer M.C. (2017) Screening cleavage of Factor XIII V34X activation peptides by thrombin mutants: a strategy for controlling fibrin architecture, Biochim. Biophys. Acta 1865 (10), 1246-1254.

3. Sery S.S., Anokhin B.A. (2012) Prospects of applying biological products of hydrocarbon oxidizing microorganisms for oily waste neutralization and processing, Ecological Bulletin of Russia 7(12), 22-25. [Russian]

4. Anokhin B.A., Maksimov A.Yu., Demakov V.A. (2011) Investigation of Dynamics and Optimization of $o$-Phthalate Degradation by Phthalate Ester-Transforming Bacteria, Vestnik Uralskoi Meditsinskoi Akademicheskoi Nauki 4/1 (38), 187-188. [Russian]

5. Ivshina I.B., Kamenskikh T.N., Anokhin B.A. (2007) Alkanotrophic Rodococci Adaptive Mechanisms Realized under Unfavorable Environment Conditions, Vestnik Permskogo Gosudarstvennogo Universiteta 10(5), 107-112. [Russian] 


\section{RECENT CONFERENCE PARTICIPATION}

1. $32^{\text {nd }}$ Annual Symposium of The Protein Society, July 2018, Boston, MA.

Anokhin B.A., Dean W.L., Maurer M.C. Activation pathway diversity as a basis for functional regulation of coagulation Factor XIII. [Poster]

2. $62^{\text {nd }}$ Annual Meeting of Biophysical Society, February 2018, San Francisco, CA.

Anokhin B.A., Stribinskis V., Dean W.L., Maurer M.C. Dissociation of Factor XIII homodimer during activation process: solution evidence weighs in on decades-long debate. [Poster]

3. $6^{\text {th }}$ Bluegrass Molecular Biophysics Symposium, May 2017, Lexington, KY.

Anokhin B.A., Maurer M.C. Probing the oligomerization of Factor XIIIA in a solution environment. [Oral presentation]

4. Gordon Research Conference Hemostasis, July 2016, Stowe, VT.

Anokhin B.A., Maurer M.C. Probing the oligomerization of Factor XIIIA in a solution environment. [Poster]

\section{Professional Membership}

Biophysical Society; The Protein Society

\section{Teaching experience}

- Chemistry 201 (General Chemistry) 2013, 2014

- Chemistry 546 (Biochemistry Laboratory) 2014, 2016

- Assisted in mentoring for undergraduate projects (7 undergraduate students) 